\title{
Aux origines de
}

l'anthropologie française. Les mémoires de la Société des observateurs de l'Homme en l'an VIII.

Textes réunis et présentés par Jean Copans et Jean Jamin Édition revue et corrigée par les auteurs en 1993

Un document produit en version numérique par Réjeanne Toussaint, ouvrière bénévole, Chomedey, Ville Laval, Québec

Page web. Courriel: rtoussaint@aei.ca

Dans le cadre de la collection: "Les classiques des sciences sociales"

Site web: http://www.uqac.ca/Classiques_des_sciences_sociales/

Une collection développée en collaboration avec la Bibliothèque

Paul-Émile-Boulet de l'Université du Québec à Chicoutimi

Site web: http://bibliotheque.uqac.uquebec.ca/index.htm 


\section{Politique d'utilisation de la bibliothèque des Classiques}

Toute reproduction et rediffusion de nos fichiers est interdite, même avec la mention de leur provenance, sans l'autorisation formelle, écrite, du fondateur des Classiques des sciences sociales, Jean-Marie Tremblay, sociologue.

Les fichiers des Classiques des sciences sociales ne peuvent sans autorisation formelle:

- être hébergés (en fichier ou page web, en totalité ou en partie) sur un serveur autre que celui des Classiques.

- servir de base de travail à un autre fichier modifié ensuite par tout autre moyen (couleur, police, mise en page, extraits, support, etc...),

Les fichiers (.html, .doc, .pdf, .rtf, .jpg, .gif) disponibles sur le site Les Classiques des sciences sociales sont la propriété des Classiques des sciences sociales, un organisme à but non lucratif composé exclusivement de bénévoles.

Ils sont disponibles pour une utilisation intellectuelle et personnelle et, en aucun cas, commerciale. Toute utilisation à des fins commerciales des fichiers sur ce site est strictement interdite et toute rediffusion est également strictement interdite.

L'accès à notre travail est libre et gratuit à tous les utilisateurs. C'est notre mission.

Jean-Marie Tremblay, sociologue

Fondateur et Président-directeur général, LES CLASSIQUES DES SCIENCES SOCIALES. 
Cette édition électronique a été réalisée par Réjeanne Toussaint, bénévole, Courriel: rtoussaint@aei.ca

Aux origines de l'anthropologie française.

Les mémoires de la Société des observateurs de l'Homme en l'an VIII.

Textes réunis et présentés par Jean Copans et Jean Jamin. Paris : Jean Michel Place, Éditeur, 1994, 213 pp. Collection : Les Cahiers de Gradhiva, no 23. Édition revue et corrigée par les auteurs en 1993. Première édition, 1978.

[Autorisation formelle accordée par MM. Jean Copans et Jean Jamin le 24 février 2011 de diffuser ce livre dans Les Classiques des sciences sociales, autorisation qui nous a été transmise par M. Jean Benoist.]

Courriels : oj.benoist@wanadoo.fr jamin@ehess.fr jean.copans@biomedicale.univ-paris5.fr

Polices de caractères utilisée : Comic Sans, 12 points.

Édition électronique réalisée avec le traitement de textes Microsoft Word 2008 pour Macintosh.

Mise en page sur papier format : LETTRE US, 8.5" $\times 11^{\prime \prime}$.

Édition numérique réalisée le 16 décembre 2011 à Chicoutimi, Ville de Saguenay, Québec. 


\section{Aux origines de l'anthropologie française.}

Les mémoires de la Société des observateurs de l'Homme en l'an VIII.

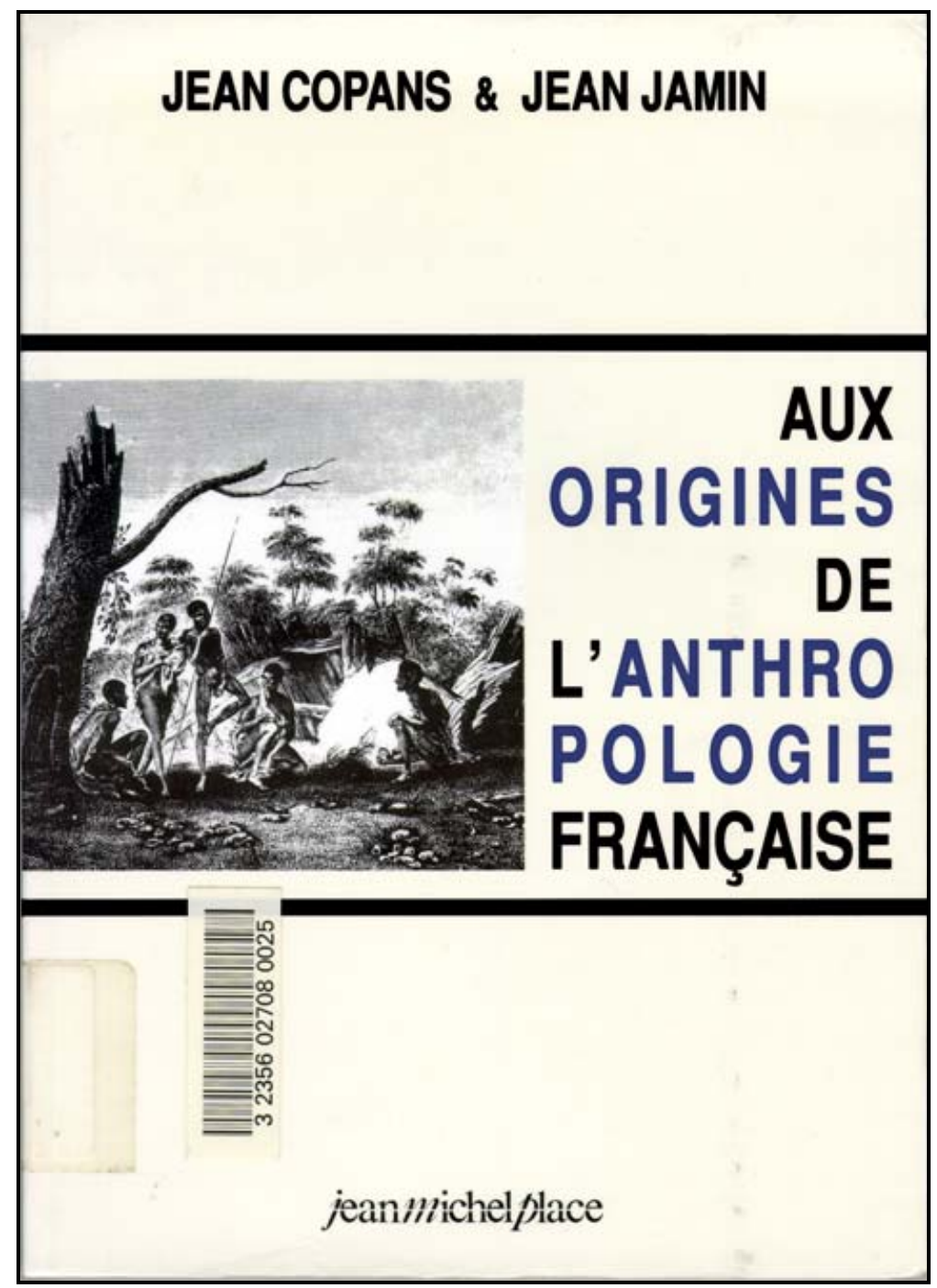

Textes réunis et présentés par Jean Copans et Jean Jamin. Paris : Jean Michel Place, Éditeur, 1994, 213 pp. Collection: Les Cahiers de Gradhiva, no 23. Édition revue et corrigée par les auteurs en 1993. Première édition, 1978. 


\section{Table des matières}

Quatrième de couverture

Présentation, par Jean Copans et Jean Jamin

Le projet anthropologique des Idéologues

La Société des observateurs de l'homme

Les écueils de l'observation "idéologique"

Les silences de la méthode

La place des Considérations... de Gérando

Un nouveau discours de la méthode

Note sur la présente édition

Texte 1. Introduction aux Mémoires de la Société des observateurs de /'Homme (1800), par Louis-François Jauffret

Texte 2. Note instructive sur les recherches à faire relativement aux différences anatomiques des diverses races d'Homme (1799), par Georges Cuvier

Texte 3. Considérations sur les diverses méthodes à suivre dans l'observation des peuples sauvages (1799), par JosephMarie de Gérando

Texte 4. Observations sur I'anthropologie ou I'histoire naturelle... (1800), par François Péron

Texte 5. Le Chinois Tchong-A-Sam (1800) par Louis-François Jauffret et Leblond

Texte 6. Rapport fait à la Société des observateurs de l'Homme sur l'enfant connu sous le nom de Sauvage de l'Aveyron (1801), par Philippe Pinel 
Texte 7. Présentation du rapport de J. M. G. Itard sur /'enfant sauvage de l'Aveyron (1801), par Joseph-Marie de Gérando

Texte 8. Mémoire sur l'établissement d'un Muséum anthropologique (1803), par Louis-François Jauffret

Texte 9. Inventaire général de tous les objets relatifs à I'histoire de l'Homme (1804), par François Péron

Texte 10. Des naturels que nous trouvions et de leur conduite envers nous (1804), par Nicolas Baudin

Texte 11. Expériences sur la force physique des sauvages (1807), par François Péron

Indications biographiques sommaires

Bibliographie des ouvrages cités 
Aux origines de l'anthropologie française. (1994)

Édition revue et corrigée par les auteurs, 1993.

QUATRIÈME DE COUVERTURE

\section{Retour à la table des matières}

Les textes réunis dans cet ouvrage constituent une partie des notes et mémoires rédigés dans le cadre de la société des observateurs de I'homme (1799-1805). Conçus et écrits pour l'expédition scientifique du capitaine Baudin « aux terres australes » en 1800, ils représentent les premières tentatives de réflexion sur les méthodes d'observation d'une discipline - l'anthropologie - qui ne devait naître et se développer qu'un siècle plus tard. Joseph-Marie de Gérando y formule avant la lettre la «règle de l'observation participante», LouisFrançois Jauffret jette les bases d'une "anthropologie comparée», François Péron voit en l'indigène un indigent, Nicolas Baudin invite déjà à s'interroger, dans le but de faire progresser la « science de l'homme », autant sur la pensée de ceux qui observent que sur les coutumes de ceux qui sont observés. 


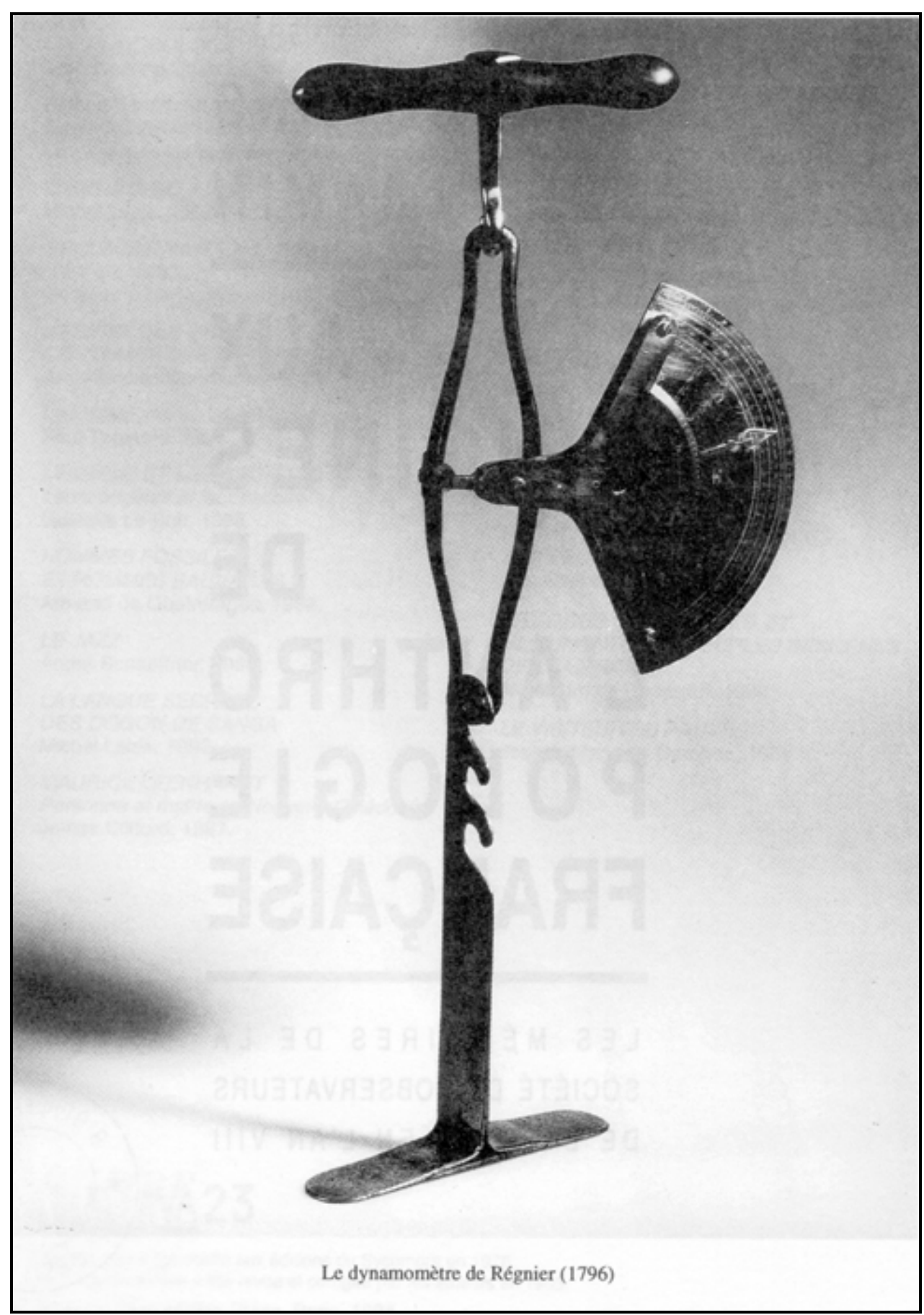

Le dynamomètre de Régnier (1796) 
Aux origines de l'anthropologie française. (1994)

Édition revue et corrigée par les auteurs, 1993.

\section{PRÉSENTATION}

Jean Copans et Jean Jamin

Paris, Amiens, janvier 1993.

Retour à la table des matières

Que les Considérations sur les diverses méthodes à suivre dans I'observation des peuples sauvages de Joseph-Marie de Gérando aient été un moment jugées irréalistes, insolites, inopportunes ou, plus radicalement, dépourvues d'intérêt, le destin bibliographique du mémoire pourrait facilement en témoigner tant celui-ci fut étouffé, voire resta clandestin.

Malgré la saveur de la langue, la pertinence des remarques et l'originalité de la démarche, les Considérations... eurent en effet peu de suites dans la littérature ethnologique. L'ancienneté de ce texte, la mention de sa date de parution in fine : 28 fructidor de l'an VIII (15 septembre 1799), son origine bien «française », que ce soit par le style ou par les idées exposées ${ }^{1}, n^{\prime} e n$ ont même pas fait une curiosité bibliographique, du moins en France.

1 Origine intéressante en ceci qu'elle permet de repenser l'histoire de l'ethnologie dite de «terrain» dont les précurseurs sont généralement recherchés 
En vérité, au moment où nous avons publié ce livre - qui contient donc le mémoire de Gérando dans son intégralité -, peu d'étudiants, peu d'enseignants, peu de chercheurs en ethnologie connaissaient cet écrit. Pareillement, et pour cause, les « maitres » de la discipline ne le mentionnaient pas. Marcel Mauss, pourtant auteur d'un manuel d'ethnographie, Marcel Griaule lui aussi auteur d'un manuel 2, Claude [8] Lévi-Strauss, Louis Dumont ou Georges Balandier - pour ne citer que les auteurs les plus connus et sans douté les plus influents de l'anthropologie française - n'y font jamais référence. L'auraient-ils ignoré ? Quant aux rares ouvrages consacrés à l'histoire de l'anthropologie, ils sont généralement tout aussi silencieux sur ce mémoire et sur la Société des observateurs de l'homme dans le cadre de laquelle il a été conçu et publié. Tout au long de l'important chapitre qu'Arnold Van Gennep a consacré à l'étude de la méthode ethnographique en France au XVIIIe siècle 3 , aucun des travaux de cette Société n'est cité. Si, dans son célèbre article de 1913 sur I'histoire de l'ethnographie en France et à l'étranger 4 , Mauss fait allusion à François Péron et à l'expédition du capitaine Baudin, ce n'est somme toute qu'une allusion... Élève de Mauss et auditeur assidu de ses cours pendant les années 30, Michel Leiris - qui fut avec nous l'un des fondateurs de la collection où devait paraître la première édition de ce livre en 1978 au Sycomore - nous confirma qu'il n'avait jamais entendu celui-ci parler des Considérations...., ni du reste des autres mémoires de la Société des observateurs de l'homme.

du côté anglo-saxon. La forme et le contenu du mémoire de Gérando ne sont pas sans rappeler, certes à première vue, Rousseau et la philosophie du siècle des Lumières ; les idées développées dans la conclusion, avec un grand élan humaniste et non sans lyrisme, ne sont pas sans évoquer la doctrine coloniale de I'« assimilation», qui avait été définie dans les articles 6 et 7 de la Constitution de l'an III.

2 Marcel MAUSS, Manuel d'ethnographie, Paris, Payot, 1989 (Ire édition due à Denise Paulme, Paris, Payot, 1947) [Livre disponible dans Les Classiques des sciences sociales. JMT.] ; M. GRIAULE, Les Méthodes de l'ethnographie, Paris, P. U. F., 1957.

3 Cf. A. VAN GENNEP, Religion, moeurs et légendes. Essais d'ethnographie et de linguistique, Paris, Maisonneuve et Larose, 1914, vol. 5, pp. 93-218.

4 Cf. M. MAUSS, «L'ethnographie en France et à l'étranger (1913)», in Oeuvres, 3, Paris, Minuit, 1969, pp. 395-435 (édition de Victor Karady). 
Il faut reconnaître que la situation n'a pas fondamentalement changé en quinze ans. L'histoire de l'ethnologie en général, de l'ethnologie française en particulier, n'apparaît pas du tout comme nécessaire à la compréhension de la nature et de l'évolution de cette discipline ${ }^{5}$. Même les auteurs de la douzaine d'ouvrages d'initiation ou de synthèse en langue française semblent hésiter quant à la lecture de ces textes ${ }^{6}$. Certes, la moitié d'entre eux évoque la Société des observateurs de l'homme, cite parfois longuement les Considérations... de Gérando tout en signalant notre édition 7 . Pourtant, quelques-uns pensent, pour des raisons qui leur sont propres, que ce dernier « détail», de nature bibliographique et pédagogique, est sans autre importance 8 . Que [9] dire, enfin, du silence complet sur ce thème dans l'ouvrage, très récent, qui constitue de fait le seul manuel de premier cycle disponible en ethnologie et anthropologie? 9. Peut-être faudrait-il s'adresser à la Mission du Patrimoine ethnologique au ministère de la

5 Par exemple le bilan publié par la revue L'Homme en 1986, « Anthropologie: état des lieux» (Paris, Le Livre de Poche, 1986) ne comporte aucun texte sur la question. Il faut lire l'Américain James Clifford pour comprendre Maurice Leenhardt, Marcel Griaule, et même Michel Leiris, du moins pour comprendre la place qu'ils ont occupée dans la fondation de l'ethnologie en France.

6 Nous pensons notamment à $T$. Todorov à qui semble échapper toute la signification du mouvement des Idéologues et qui tend à réduire les Considérations... de Gérando à un discours ethnocentrique et quasi colonialiste. Cf. T. TODOROV, Nous et les autres. La réflexion française sur la diversité humaine, Paris, Le Seuil, 1989, pp. 30-31, 40-41 et 339-342.

7 Cf. M. KILANI, Introduction à l'anthropologie, Lausanne, Payot, 1992, p. 239 ; F. ROGNON, Les Primitif, nos contemporains, Paris, Hatier, 1988, pp. 80, 138141 ; I. SCHULTE-TENCKHOFF, La Vue portée au loin. Une histoire de la pensée anthropologique, Lausanne, éditions d'en-bas, 1985, p. 34 ; A. C. TAYLOR, "Les modèles d'intelligibilité de l'Histoire», in PH. DESCOLA, G. LENCLUD, $C$. SÉVERI et A. C. TAYLOR, Les Idées de l'anthropologie, Paris, Armand Colin, 1988, p. 165.

8 Cf., par exemple, F. LAPLANTINE, Clefs pour I'anthropologie, Paris, Seghers, 1987, pp. 57-58, 208, et G. LENCLUD, « Le Grand Partage ou la tentation ethnologique », in G. ALTHABE, D. FABRE et G. LENCLUD (sous la direction de), Vers une ethnologie du présent, Paris, éditions de la Maison des Sciences de I'Homme, 1992, pp. 13 et 17.

9 Cf. PH. LABURTHE-TOLRA \& J.-P. WARNIER, Ethnologie, Anthropologie, Paris, Presses universitaires de France, 1993. 
Culture pour que la restitution et la valorisation du patrimoine et des traditions ethnologiques françaises soient enfin considérées avec attention et sérieux! 10

\section{Le projet anthropologique des Idéologues}

\section{Retour à la table des matières}

Au-delà du jeu des circonstances et des accidents bibliographiques, il semble bien que les membres de la Société des observateurs de I'homme se rattachant pour la grande majorité d'entre eux au mouvement dit de l'Idéologie aient été les victimes d'une véritable « conspiration du silence » en partie due à la péjoration et à la méprise dont ont été sujets ces «malheureux» philosophes, penseurs et écrivains du Directoire puis du Consulat, rabaissés au rang d'« idéologues». Ce furent tout d'abord celles de Bonaparte qui, en 1801, les qualifia ainsi, irrité par le groupe de pression et d'opposition qu'ils constituaient au Sénat et au Tribunat: " Des rêveurs, des phraseurs, des métaphysiciens, bons à jeter à l'eau! » s'était-il écrié. Bonaparte ne faisait là que consommer une rupture et exprimer d'une façon cinglante un désaccord politique avec ceux qui, pourtant, l'avaient porté au pouvoir et qui, globalement, avaient approuvé le 18 brumaire. En 1803, profitant de la réorganisation de I'Institut de France, Bonaparte supprima la seconde classe de l'Institut, celle des Sciences morales et politiques où siégeaient la plupart des membres du groupe et d'où ils tenaient leur légitimité. Dans sa Correspondance (vol. XXV), Bonaparte, par irritation, en vient à donner au mot «Idéologues» son sens moderne: "C'est à l'Idéologie, écrit-il, à cette ténébreuse métaphysique qui, en recherchant avec subtilité les causes premières, veut sur ces bases fonder la législation des peuples, au lieu d'approprier les lois à la connaissance du cœur humain et aux leçons de l'histoire, qu'il faut attribuer tous les malheurs qu'a éprouvés notre belle France. » 
Ce furent, ensuite, celles de Karl Marx ${ }^{11}$ qui crut percevoir dans leurs œuvres le prototype même de ce système de pensée qui, consciemment ou non, tend à justifier sous un raisonnement global une forme particulière d'organisation sociale et politique. Parce qu'elle l'aurait été au départ, l'Idéologie serait donc toujours mystificatrice. Les Idéologues, quant à eux, n'ont jamais revendiqué ce nom qui, depuis Bonaparte et à sa suite, leur a été attribué ; ils se désignaient sous celui d'idéologistes, autrement dit: analystes de l'entendement humain.

[10]

Incontestablement donc, les Idéologues sont des auteurs maudits du domaine français. Georges Gusdorf 12 n'hésite pas à voir en eux et dans le courant qu'ils ont représenté une "génération perdue » qui a été jetée aux poubelles de l'histoire. Jean Gaulmier 13 (l'éditeur et le biographe de Volney) s'étonne de cette éclipse totale de la pensée qui aurait eu lieu de 1778 (mort de Rousseau et de Voltaire) à 1815.

Il est vrai que jusqu'en 1891, aucune étude d'ensemble ne leur a été consacrée. Dans sa thèse touffue, parfois confuse - mais qui reste l'ouvrage de référence - François Picavet va tenter de lever le voile ${ }^{14}$. En vain! La période révolutionnaire, pré et postrévolutionnaire demeure une période creuse dans I'histoire de la vie intellectuelle française, - une période terne sinon ténébreuse entre

11 Cf. K. MARX, L'idéologie allemande, Paris, Éditions sociales, 1968 (édition de Gilbert Badia), notamment pp. 43-45 et pp. 260-263. [Livre disponible dans Les Classiques des sciences sociales, JMT.] Sur I'histoire du mot « idéologie», voir l'ouvrage, quelque peu polémique, de R. BOUDON, L'idéologie ou l'origine des idées reçues, Paris, Fayard, 1986.

12 Cf. G. GUSDORF, La Conscience révolutionnaire. Les Idéologues, Paris, Payot, 1978.

13 J. GAULMIER, L'idéologue Volney, Thèse de doctorat, Beyrouth, 1951; J. GAULMIER (éd.): Volney, Voyage en Egypte et en Syrie, Paris, Mouton, 1959 : J. GAULMIER (éd.) : Volney, La loi naturelle. Leçons d'histoire, Paris, Garnier, 1980.

14 Cf. F. PICAVET, Les Idéologues. Essai sur I'histoire des idées et des théories scientifiques, philosophiques, religieuses, etc., en France depuis 1789, Paris, Alcan, 1891. 
française, - une période terne sinon ténébreuse entre l'éclat des Lumières et les tourments du romantisme. Ce ne sera qu'en 1964, avec l'article de l'Américain G.W. Stocking 15, et surtout à partir de 1968 avec I'Italien Sergio Moravia ${ }^{16}$, que les Idéologues seront reconsidérés ou tout simplement considérés. On peut noter que ces deux auteurs sont "étrangers» et que leurs contributions n'ont jamais été traduites jusqu'à ce jour. Des travaux dus à des auteurs français vont suivre, mais à pas comptés et d'une manière quelque peu embarrassée, hésitante, partagée en tout cas entre la réhabilitation parfois naive, parfois grandiloquente (Georges Gusdorf), et l'évaluation quelque peu hautaine, philosophique et critique (Michel Foucault). Outre les travaux de Jean Gaulmier sur Volney, ce sera d'abord une thèse volumineuse de Marc Régaldo 17 sur ce qui fut l'organe d'expression des Idéologues, la revue La Décade philosophique qui a paru de 1794 à 1807 (alors interdite par Napoléon 1er); puis l'ouvrage déjà cité de Gusdorf en 1978 ainsi que la première édition de ce livre: un numéro spécial de la revue Histoire, épistémologie et langage en 1982 consacré aux «Idéologues et les sciences du langage 18 (sous la direction de Claude Désirat et de Tristan Hordé); enfin le livre de Claude Nicolet sur L'Idée républicaine en France ${ }^{19}$, - celui-ci voyant en eux non seulement les inventeurs de l'idée républicaine mais les premiers artisans de l'idéal républicain. À juste titre, Nicolet insiste sur l'originalité de la démarche des Idéologues qui associent [11] la pensée républicaine à une philosophie de la connaissance. Plus récemment, Patrick Quentin a publié un ouvrage bien informé sur les origines, les développements et

15 Cf. G. W. STOCKING, « French Anthropology in $1800 »$, Isis, Vol. LV, 2, $n^{\circ} 180$, 1964, pp. 134-150.

16 Cf. S. MORAVIA, II Tramonto del l'illuminismo. Filosofia e politica nella societa francese (1770-1810), Bari, Laterza, 1968 ; La Scienza dell homo nel Stettencento, Florence, La nuova Italia, 1970 ; II pensiero degli ideologues, Florence, La nuova Italia, 1974.

17 Cf. M. RÉGALDO, Un milieu intellectuel : La Décade philosophique (1794-1807), thèse de doctorat d'État, Université de Lille, 1976.

18 C. DÉSIRAT et T. HORDÉ (éds.), «Les Idéologues et les sciences du langage », Histoire, épistémologie et langage, T. 4, fasc. 1, 1982.

19 CL. NICOLET, L'idée républicaine en France. Essai d'histoire critique, Paris, Gallimard, 1982. 
les critiques de l'Idéologie à partir surtout d'une analyse de l'œuvre de Destutt de Tracy 20. Bref, peu de choses en vérité... qui laissent entièrement ouverte la question de ce qui est tout à la fois une discipline, une conception savante du monde et de la société, un groupe d'intellectuels, un dispositif pédagogique et un corps législatif. Que penser en effet et quoi faire des Idéologues qui semblent tant embarrasser I'histoire des idées en France?

Le bilan de leurs activités n'est cependant pas mince:

- au niveau institutionnel, on leur doit la création, l'organisation et, souvent, l'animation des Écoles centrales («ancêtres» de nos lycées), des Écoles normales et des Écoles spéciales (polytechnique, langues orientales, Muséum, etc.):

- au niveau intellectuel, on leur connaît un nombre considérable de travaux, articles, leçons, mémoires et manuels touchant à tous les domaines de la pensée et du savoir (les Idéologues étaient volontiers polygraphes), mais dont peu ont subsisté ou se sont imposés. Les rééditions ont été rares, tout aussi rares les citations ou les renvois à leurs cuvres. De sorte que les textes originaux restent difficilement accessibles. Certes, beaucoup de ces écrits ont été de circonstance en vue d'un accès direct à la vie publique que leurs auteurs ont semblé vouloir rechercher et privilégier au point d'ailleurs que le texte fondateur et programmatique de Destutt de Tracy, les Éléments d'idéologie, fut à l'origine un manuel conçu à l'usage des Écoles centrales. Une telle pensée « en situation et en action» fait toute la différence avec la génération précédente des Lumières. En somme, une telle attitude témoigne du rapport de la pensée idéologique avec la pratique politique et sociale qui a pu faire se méprendre Marx, par exemple, sur la portée de l'Idéologie et sur l'aspect mystificateur qu'il crut y découvrir.

20 Cf. P. QUENTIN, Les Origines de l'idéologie, Paris, Economica, 1987 ; J.-P. COTTEN, «La politique de la philosophie. Note sur la France du début du XIXe siècle », La Pensée, $n^{\circ} 274,1990$, pp. 77-86. 
De fait, aucune cuuvre maîtresse, aucun maître à penser ne signale le courant idéologique. Cela tient en partie à des aspects théoriques que nous évoquerons plus bas, c'est-à-dire à la définition même de l'Idéologie qui se veut plus une méthode qu'une doctrine; en partie à la conception même que se forgèrent les Idéologues de l'entreprise du savoir, laquelle les conduisit à inventer de nouvelles formes de construction, d'accumulation et de diffusion de celui-ci, les unes administratives qui ne sont pas sans évoquer la littérature «grise» de nos actuelles sociétés d'étude, les autres pédagogiques qui conférèrent à la plupart de leurs travaux écrits le statut de manuels; en partie sans doute la plus importante - à leur implication dans les affaires de la cité, qui eut finalement raison de leur espérance de vie puisque le courant comme le groupe se dispersent en 1803, après la suppression par Bonaparte du Tribunat et de la classe des Sciences morales et politiques de l'Institut.

Pour la plupart rescapés de la Terreur, thermidoriens, pour certains inspirateurs [12] sinon rédacteurs de la Constitution de I'an VIII, les Idéologues ont été à la fois des penseurs et des législateurs, des savants et des hommes politiques. Ce qui donc les caractérise, c'est la proximité avec le pouvoir politique, la participation à l'exercice de ce pouvoir dont ils se veulent, au nom de la raison et forts des apports de ces sciences de l'homme qu'ils cherchent à fonder, les conseillers et les censeurs, les guides et les législateurs. En outre, et du fait de leur rôle prépondérant dans la mise en place d'un nouveau système d'enseignement qui participe lui aussi d'un projet politique selon l'équation : éduquer les enfants = gouverner les adultes qu'ils deviendront (l'éducation étant alors conçue comme un moyen d'émancipation et un principe de gouvernement), les Idéologues consacrent l'apparition dans la culture française du personnage de l'intellectuel qu'on appellera plus tard engagé. Ils ont donc occupé cette position peu confortable d'être à la fois les témoins, les penseurs et même les artisans de mutations sociales profondes; position qui, pour la première fois peut-être dans l'histoire, conduisit à poser, comme le 
souligne Gérard Leclerc 21 , le problème de l'interaction entre un système d'observation sociale et le système social observé. Si bien que le projet anthropologique se trouve inscrit dans leur situation de classe intellectuelle. Michel Foucault a analysé dans Les Mots et les cho$\operatorname{ses}^{22}$ le basculement d'une épistémè à l'autre, le passage de la représentation de l'âge classique à la positivité du XIXe siècle et de la connaissance moderne. Les thèses de cet ouvrage sont bien connues, mais il nous a semblé nécessaire de signaler quelques remarques qui éclairent singulièrement la situation transitoire des Idéologues et par conséquent des textes de la Société des observateurs de l'homme. Deux thèmes sont décisifs aux yeux de Foucault : le double rapport du langage à la nature et de chacun de ces domaines à lui-même. "La théorie de l'histoire naturelle, écrit-il (p. 170) n'est pas dissociable de celle du langage ». Mais les changements qui s'opèrent ont une portée plus générale (pp. 220-221). Ainsi « [...] vers la fin du XVIIIe siècle [...] négativement, le domaine des formes pures de la connaissance s'isole, prenant à la fois autonomie et souveraineté par rapport à tout savoir empirique, faisant naître et renaître indéfiniment le projet de formaliser le concret et de constituer envers et contre tout des sciences pures: positivement les domaines empiriques se lient à des réflexions sur la subjectivité, l'être humain et la finitude, prenant valeur et fonction de philosophie, aussi bien que de réduction de la philosophie ou de contre-philosophie. » (p. 261). Le courant des Idéologues est l'élaboration extrême du point de vue classique et pourtant il éclate déjà en permettant une ouverture positive. Mais c'est ailleurs, avec Kant, qu'apparaît le signe constitutif des bases de ce changement 23 .

21 Cf. G. LECLERC, L'Observation de I'homme, Paris, Seuil, 1979.

22 Cf. M. FOUCAULT, Les Mots et les choses, Paris, Gallimard, 1966. Il faut tout de même souligner le caractère essentiellement «philosophique » de la démonstration de Foucault.

23 Il conviendrait d'entreprendre une lecture sérieuse de la place de Kant et de la philosophie d'inspiration kantienne en général dans l'inconscient de la tradition anthropologique. On sait ce que Claude Lévi-Strauss a avoué à Paul Ricœur sur ce point! 
[13]

L'Idéologie débouche sur une science totalisante de l'homme:

«Chez Destutt ou Gérando, I'Idéologie se donne à la fois comme la seule forme rationnelle et scientifique que la philosophie puisse revêtir et unique fondement philosophique qui puisse être proposé aux sciences en général et à chaque domaine singulier de la connaissance. Science des idées, l'Idéologie doit être une connaissance de même type que celles qui se donnent pour objet les êtres de la nature, ou les mots du langage, ou les lois de la société. Mais dans la mesure même où elle a pour objet les idées, la manière de les exprimer dans des mots, et de les lier dans des raisonnements, elle vaut comme la grammaire et la logique de toute science possible. [...] Ce qui, lu dans un sens, apparaît comme la généralité la plus mince de la pensée, apparaît, déchiffré dans une autre direction, comme le résultat complexe d'une singularité zoologique: "On n'a qu'une connaissance incomplète d'un animal, si l'on ne connaît pas ses facultés intellectuelles. L'idéologie est une partie de la zoologie, et c'est surtout dans l'homme que cette partie est importante et qu'elle mérite d'être approfondie [Destutt de Tracy]." L'analyse de la représentation, au moment où elle atteint sa plus grande extension touche par son bord le plus extérieur un domaine qui serait à peu près - ou plutôt qui sera, car il n'existe pas encore - celui d'une science naturelle de I'homme. » 24

Ce qui nous intéresse c'est cette possibilité d'une positivité des objets du savoir. Et cette possibilité est malgré tout définie par de nouvelles conditions d'observation. Mais l'analyse de Foucault est trop préoccupée de l'ordre du discours et elle sous-estime le problème des pratiques. Bien sûr les pratiques se doivent d'être pensées avant comme après, mais il est impossible d'en faire abstraction.

24 M. FOUCAULT, op. cit., 1966, pp. 253-254. 
La «théorie» des Idéologues - l'Idéologie - est d'origine assez composite bien qu'elle se veuille unitaire. Elle se caractérise d'abord par le double rejet de la métaphysique et du dualisme, puis par une sorte de passion du fait, de l'expérience, qui annonce le positivisme. Elle est surtout marquée par le sensualisme, le sensationnisme devraiton dire, de Condillac, le matérialisme de d'Alembert, l'athéisme radical de d'Holbach, l'empirisme de Locke sans oublier le libéralisme de Smith qui eut I'influence que l'on sait sur l'économiste du groupe, Jean-Baptiste Say.

Cette notion d'Idéologie fut introduite par Destutt de Tracy dans un mémoire sur la faculté de penser qu'il lut à l'Institut en 1796, dans le cadre de la classe des Sciences morales et politiques. Sous ce terme, il entendait désigner une nouvelle branche de la science qui s'occupât des idées, des facultés intellectuelles, de leur formation et développement, des moyens de les connaître et de les analyser. Telle que définie par Destutt de Tracy, I'Idéologie regroupe la science de la formation des idées ou idéologie proprement dite, la science de leur expression ou grammaire, celle de leur combinaison et déduction ou logique. Mais au-delà de cette définition technique, Destutt de Tracy concevait un ensemble interdisciplinaire qui faisait de l'Idéologie la théorie des théories pour reprendre son expression, en ce sens qu'elle [14] visait à réunir sous une problématique et une méthodologie communes - cette dernière inspirée des sciences de la nature - des régions jusqu'alors distinctes du savoir de l'homme sur l'homme. L'Idéologie se présenta en somme comme une entreprise de remembrement à la fois intellectuelle et institutionnelle, comme une sorte d'assemblée constituante d'une nouvelle philosophie en faisant valoir notamment que des disciplines aux territoires apparemment aussi fixés que la grammaire, la logique, la physiologie, les sciences économiques, politiques et historiques etc., relevaient d'un même champ de scientificité qu'aujourd'hui l'on appellerait sciences de l'homme. 
"On ne saurait comparer les faits qu'après les avoir connus, écrivait Destutt de Tracy dans ses Éléments d'idéologie 25 , et on ne peut découvrir les lois générales qui régissent ces faits, qu'après les avoir comparés. Cela nous explique aussi pourquoi la science qui nous occupe, celle de la formation des idées, est si nouvelle et si peu avancée: puisqu'elle est la théorie des théories, elle devait naître la dernière. Ceci, au reste, ne doit pas nous faire conclure que les théories en général, et notamment l'idéologie, soient inutiles; elles servent à rectifier et épurer les diverses connaissances, à les rapprocher les unes aux autres, à les rattacher à des principes plus généraux, enfin à les réunir par tout ce qu'elles ont de commun. »

De ce point de vue, l'Idéologie ne constitue pas une école de pensée stricto sensu. Sa préoccupation majeure est de lier entre elles des disciplines jusqu'alors sans relations institutionnalisées et qui, chacune, simultanément - comme chez l'hygiéniste et médecin Cabanis - ou successivement - comme chez l'aliéniste Pinel - seront conviées pour répondre à cette question, point de départ de l'Idéologie: Qu'est-ce que penser? Qu'est-ce que penser l'homme, la nature, la société, I'histoire?

De ce point de vue également, l'Idéologie pourrait être perçue comme un prolongement ou, disons, comme un sous-ensemble - celui des sciences humaines - de l'entreprise encyclopédique. Or, les idéologues ne se reporteront guère ni ne se référeront explicitement à l'Encyclopédie. À cela quatre raisons, comme le soulignent Tristan Hordé et Claude Désirat dans un ouvrage malheureusement inédit jusqu'à présent 26 :

Cf. DESTUTT DE TRACY, Éléments d'idéologie, Paris, Vrin, 1970 (réimpression de l'édition de 1801).

26 CL. DÉSIRAT \& T. HORDÉ, Du bon usage du sens. Textes linguistiques et ethnographiques de Volney, s.l.n.d. 
- les survivants de l'Encyclopédie ne sont pas tous, loin s'en faut, des sympathisants de la Révolution:

- l'ordre alphabétique occulte la hiérarchie des objets et l'organisation des disciplines:

- la subordination des arts aux sciences, essentielle pour Destutt de Tracy, n'y apparaît pas assez nettement :

- enfin, le livre n'est plus la seule forme de diffusion possible des idées; les cours et conférences, les séminaires et enseignements, les réunions et associations savantes seront privilégiées par les Idéologues qui essaieront de donner de nouvelles conditions institutionnelles à la diffusion du savoir par le biais de l'Institut et des Écoles centrales et spéciales.

[15]

Le savoir ne se constitue pas - pas plus qu'il ne se développe - par effet de masse et de concentration, mais il se forme par réseau, par diffusion ou, expressément, par irrigation. À ce titre, le choix de moyens disons soft de sa diffusion (articles, conférences, leçons) a comme contrepartie le choix de moyens mettons hard de son acquisition (questionnaires, manuels) qui procèdent d'une conception collective en même temps que démocratique - maintes fois défendue par les Idéologues - de l'organisation du savoir: les observateurs doivent être interchangeables et leur observation déconnectée de toute idiosyncrasie, d'où la nécessité de la régler et de la multiplier afin que les matériaux s'accumulent et s'enrichissent. Il s'agit bien, pour reprendre une idée d'Hélène Metzger 27 , d'une période intense de « socialisation de la Science », où la science qui se faisait devait se mettre à la portée de tous.

Idéalement, penser l'homme nécessite que tous les hommes pensent l'homme en même temps selon les mêmes tables. A la notion d'auteur, les Idéologues tentent de substituer celle d'enquêteur et d'informateur. En 1803, Volney s'adressera au ministère de l'Intérieur pour obtenir la mise en place d'un réseau d'informateurs locaux par 
l'intermédiaire des préfets et sous-préfets, Pour la première fois sans doute dans l'histoire des idées et de leur diffusion, le rôle principal sera donné non point au livre mais à la revue, - telle La Décade philosophique qui, pendant plus de dix ans, avec ses quelques six cents abonnés, deviendra l'organe privilégié de cette régulation et de cette conception qu'on pourrait dire «interactive» du savoir de l'homme sur l'homme. Ceci conduit à « désacraliser» l'auteur et l'écrit (on peut d'ailleurs s'en rendre compte, en quelque sorte par défaut, dans la désinvolture qu'ont pu afficher les Idéologues vis-à-vis d'autres auteurs et de la citation des sources, non seulement se copiant les uns les autres mais pillant sans vergogne), - ceci conduit, du moins, à donner à l'écrit un caractère provisoire, révisable, réfutable.

Bref, en affirmant que de nos sensations naissent nos idées puis, en faisant de la genèse de celles-ci l'objet d'une observation rigoureuse, Destutt de Tracy leur accorde du même coup une positivité, voire une laicité. La pensée, de fait, de raison et de méthode, se trouve elle aussi « désacralisée », « contextualisée», «naturalisée ». L'Idéologie embrasse non seulement l'univers de la langue, du discours et de l'entendement mais leur enracinement biologique. L'Idéologie se présente donc à la fois comme une épistémologie, une méthode et une pédagogie: elle renonce à toute explication de type transcendantal et $s$ 'appuie sur les faits qu'elle va chercher à organiser et dont elle entend édicter les règles d'observation pour rendre compte des lois de leur connaissance tout autant que de celles qui règlent leurs appariement et fonctionnement. Au sens fort, l'Idéologie se veut une hypothèse sur le monde et l'homme suivant l'exemple, sans doute, de la Révolution française dont elle est issue et qui s'est voulue une hypothèse sur l'organisation sociale et politique du monde. 


\section{La Société des observateurs de l'homme}

\section{Retour à la table des matières}

Peut-on parler d'un projet anthropologique des Idéologues?

- Oui, si l'on admet que la Société des observateurs de l'homme, fondée en 1799 par Louis-François Jauffret et réunissant la plupart des Idéologues en place, joua le rôle d'une section « anthropologique » de la deuxième classe de l'Institut. Dans l'article premier de ses statuts, il est déclaré que la Société consacre exclusivement ses travaux à l'étude de l'homme physique, intellectuel et moral sous toutes les latitudes et dans toutes les périodes de l'histoire. Formée de médecins (Pinel, Cabanis), de linguistes (Sicard, Leblond), de philosophes (Destutt de Tracy, Laromiguière), d'historiens (Volney, Millin, Levesque, - ce dernier, auteur d'une remarque célèbre: "Les sorciers furent les premiers médecins, les premiers prêtres et les premiers théologiens, et même les premiers philosophes »), formée également de naturalistes (Cuvier, Geoffroy Saint-Hilaire, Jauffret), d'essayistes et «publicistes» (Gérando, Ginguéné), de voyageurs (Bougainville, Baudin, Levaillant) et du premier «anthropologiste» ainsi nommé, François Péron, (au total cinquante membres titulaires et cinquante membres correspondants), la Société des observateurs de l'homme concrétise et institutionnalise le projet de l'Idéologie: déterminer une genèse de toutes les formes de la connaissance humaine à partir d'une méthodologie unitaire. La Société se donne pour tâche de recueillir beaucoup de faits, et rien que les faits, d'étendre et de multiplier les observations sociales et culturelles afin de jeter les bases d'une anthropologie comparée (l'expression est de Jauffret) qui intègre dans son projet scientifique aussi bien l'étude des anciens peuples (domaine des Antiquités) que celle des peuples indigènes (sauvages) ou indigents (sourds-muets, pauvres). Cette anthropographie des différentes régions (l'expression est toujours de Jauffret) devrait aboutir à la création d'un Muséum spécial - véritable ancêtre du Musée de 
I'Homme - qui présenterait les divers objets relatifs aux travaux dont la Société s'occupe, et en particulier (nous citons l'article 2 des statuts) «tous les produits de l'industrie des sauvages, tous les objets de comparaison qui peuvent servir à faire connaître les variétés de l'espèce humaine, ainsi que les mours et les usages des peuples anciens et modernes».

- Oui, si l'on considère qu'un des buts de l'idéologie est d'analyser les lois naturelles de l'entendement humain dans ses cuvres (non seulement dans la pensée mais aussi dans les institutions et les industries), dans sa géographie et dans son histoire. Le premier domaine de l'Idéologie - l'analyse des sensations et de la « génération » des idées - débouche directement sur une ethnographie. Il s'agit d'aller là où cette genèse peut s'appréhender le mieux car le plus simplement: chez les indigènes ou les indigents, chez les peuples campagnards ou montagnards, chez des êtres supposés simples et élémentaires qui constituent ainsi une sorte de laboratoire idéologique et sociologique comme le précisera Gérando dans ses Considérations... (cf. infra): «Ici, lés générations n'ayant exercé qu'une très légère influence, nous nous trouvons [17] en quelque sorte reportés aux premières époques de notre histoire: nous pouvons établir de sûres expériences sur l'origine et la génération des idées, sur la formation et les progrès du langage, sur l'enchaînement qui existe entre ces deux ordres d'opérations. »

Cette problématique suppose une conception unitaire de l'espèce humaine, une conception moniste de l'homme où, pour reprendre la perspective de Cabanis, le physique et le moral ne sont point en opposition mais en interaction. L'Autre, le Sauvage, est un Même mais un Même simplifié, tout comme le Fou est un Même déréglé, I'Indigent un Même anémié. Leur observation, leur connaissance sont perçues comme utiles, ne serait-ce que pour mieux se comprendre soi-même. A l'image de Pinel qui entend montrer aux sages comment ils pensent en expliquant comment les fous déraisonnent, Gérando, en recommandant à l'observateur d'apprendre comment le sauvage parle et pense, attend que le civilisé découvre comment il a appris à parler et à penser. Les 
différences de races et d'environnements ne sont donc nullement déterminantes; elles infléchissent tout au plus les règles de combinaison des idées, limitent leur nombre, spécifient leurs points d'application, mais elles n'altèrent pas leur formation:

«Les sauvages, écrit Destutt de Tracy dans Éléments d'idéologie, nous donnent souvent lieu d'admirer que des hommes si peu éclairés fassent des combinaisons si fines et que, les faisant, ils soient tout à fait incapables d'en faire d'autres qui nous paraissent moins difficiles. Dans les sociétés civilisées, ajoute-t-il, la classe qui a les communications les moins étendues et les moins variées offre des phénomènes analogues. »

- Oui enfin, si l'on tient compte de la place que les Idéologues assignaient à la science de l'homme et des moyens tant institutionnels qu'intellectuels qu'ils mirent en œuvre pour la constituer et dont certains ont déjà été évoqué.

Une fois restaurée l'initiative humaine et son rôle dans I'histoire (ainsi que venait de le faire la Révolution française), il devient possible et nécessaire d'étudier ses principes et son évolution. Tel est le postulat de départ, comme le soulignent Désirat et Hordé dans leur ouvrage déjà cité. Tous les résultats des recherches que peuvent entreprendre les différentes branches de la Science devront être utilisés pour répondre à cette question: Quels sont les moyens intellectuels propres à assurer le bonheur des hommes? Ce lien posé entre la pensée et l'action implique une science du politique. En effet, une des conditions émises par les Idéologues à la possibilité de l'observation anthropologique comme à sa validité est de nature à proprement parler politique : elle suppose que /'autre soit considéré comme un sujet libre et égal en droit, qui plus est, soit considéré lui-même comme un sujet de I'Histoire. Pour Gérando comme pour Volney les motivations de l'enquête doivent être exposées et explicitées: il faut que l'autre l'observé - reconnaisse le bien-fondé du projet de l'enquête: par conséquent il convient de le convaincre de s'y prêter. Enquêteur cer- 
tes, mais aussi et surtout « visiteur » (c'est du reste sous ce titre Le Visiteur du pauvre que Gérando publiera près de vingt ans plus tard son étude sur la pauvreté en France), l'observateur de l'homme se veut un concitoyen: "Comment se flatter, écrit Gérando [18] dans ses Considérations... de bien observer un peuple qu'on ne sait pas comprendre et avec lequel on ne peut s'entretenir? Le premier moyen pour bien connaître les sauvages, est de devenir en quelque sorte comme l'un d'entre eux et c'est en apprenant leur langue qu'on deviendra leur concitoyen. » 28

L'objet de l'enquête se voit donc accordé le statut de sujet ; son droit à la parole, pas seulement à la langue mais aussi aux idées, est reconnu comme lui est reconnue la même capacité de jugement qu'on se reconnaît, - le même droit à connaître les faits et à les interpréter. Contre toute attente ou contre certaines idées reçues, il semble bien que ce soit l'individualisme qui se trouve ici au fondement du projet anthropologique : il ne suffit pas de poser des questions, encore faut-il que celui à qui on les pose accepte d'y répondre, ce qui nécessite de reconnaître son libre arbitre, et par conséquent, son individualité. La relation d'observation est d'abord une affaire d'individu à individu, une affaire entre soi et l'autre, une affaire de contrat. Et c'est dans ce processus d'interlocution que peut se dégager un sens commun où la connaissance de l'homme, de soi par l'autre et de l'autre par soi, s'étant accrue, sa condition, disons son éducation et son gouvernement pour reprendre les termes des Idéologues, devrait s'en trouver facilitée sinon améliorée. Cette vision à l'évidence optimiste - mais qui sera lourde de conséquences en situation coloniale - de ce qu'on peut d'ores et déjà appeler la relation ethnographique trouve sa justification théorique dans l'Idéologie même qui, affirmant le primat de l'individu, refuse de reconnaître l'origine de ses variétés et de ses différences dans la nature, les climats, l'environnement, ou dans la couleur de la peau. L'histoire et l'état de la société - ce qui fait l'homme - peuvent seuls en rendre compte. Le principe des mours trouve ses fondements

28 Cf. sur Gérando les articles récents de J.-F. BRAUNSTEIN, « Le sourire du pâle Vasco ou le voyage des Idéologues», Oui, la philosophie, n³, 1984, pp. 4655 : «De Gérando, le "social" et la fin de l'Idéologie», Corpus, $n^{\circ} 14-15$, pp. 197-215. 
dans la nature de l'homme, qui est d'être avant tout un être de langage et d'idées, un sujet de mémoire et de représentations. Cette vision repose également sur ce refus radical qu'ont toujours manifesté les Idéologues de distinguer « la pratique scientifique de la pratique ordinaire du monde » 29 ainsi que l'a très bien formulé l'Idéologue, philosophe et historien, Joseph-Dominique Garat : «Le laboureur qui réfléchit sur sa charrue, réfléchit de la même manière que Newton sur le système du monde. »

\section{Les écueils de l'observation "idéologique"}

\section{Retour à la table des matières}

Les cinquante-sept pages de l'édition originale du mémoire de Gérando furent donc publiées sous forme de brochure par la Société des observateurs de l'homme, laquelle, du fait de son existence éphémère (1799-1805), laissa peu d'archives. La bibliothèque du Musée de l'Homme à Paris, qui fut et reste encore un lieu privilégié pour la consultation des documents et travaux ethnologiques, ne possède qu'une photocopie [19] de cet écrit 30 ; ce qui signifie que son entrée au catalogue, par conséquent la possibilité offerte aux ethnologues d'en lire tout ou partie serait récente. De telles conditions ont certes pu causer son oubli et être à l'origine de sa méconnaissance.

Ce mémoire ainsi que d'autres, issus de la Société des observateurs de l'homme, ont été cependant exhumés par Ernest-Théodore Hamy ${ }^{31}$ et publiés par les soins de Paul Topinard et de Georges Her-

29 Cf. CL DÉSIRAT \& T. HORDÉ, op. cit., s.I.n.d.

30 C'est à cette photocopie que fait allusion J. POIRIER dans son Histoire de I'ethnologie (Paris, P.U.F., 1969, p. 22) ; mais elle n'apparaît pas dans le fichier systématique de la bibliothèque du Musée de l'Homme à l'entrée « Guides d'enquête». L'imprimé original du mémoire est conservé à la bibliothèque du Muséum national d'Histoire naturelle sous la cote : M. M. 6. L.

31 Ernest-Théodore Hamy (1842-1908) fut le fondateur et le premier conservateur du Musée d'ethnographie du Trocadéro (1877), et membre de la Société d'anthropologie de Paris (fondée par Paul Broca en 1859). Hamy dirigea la Revue d'ethnographie qui parut de 1882 à 1889 . Outre la découverte de quelques 
vé 32 , d'abord en 1883, puis en 1909, dans la Revue d'anthropologie et dans les Bulletins et Mémoires de la Société d'anthropologie de Paris. Il était donc, sous une forme plus commode et plus accessible, de nouveau disponible à l'époque où Durkheim et Mauss animaient l'École française de sociologie et la revue L'Année sociologique, jetaient les bases théoriques et méthodologiques de l'anthropologie française, tentaient de penser le terrain et de régler l'observation ethnographique. Le fait qu'ils ne s'y soient jamais référer donne à penser sur la nature des rapports entre ces deux « écoles » anthropologiques - disons, pour faire court, l'école « naturaliste » et l'école «culturaliste » (ou plus exactement sociologique) - qui, ayant pour objet l'étude de I'homme, entre autres celle de l'homme "primitif», ne le prenaient pas par les mêmes "bouts » et ne cherchaient visiblement pas à les raccorder. L'ironie du sort voulut qu'en plus, Gérando, attaché donc comme les Idéologues de son époque à l'étude des signes, du langage et de la pensée humaine, fut redécouvert par ceux qui ne connaissaient alors que le premier sens du terme sémiologie, c'est-à-dire les anthropologues physiques, de formation médicale pour la plupart, et qui, sous la férule de Paul Broca, s'étaient constitués en Société d'anthropologie de Paris.

Dans un article publié en 1956, justement consacré à la Société des observateurs de l'homme, Marcelle Bouteiller 33 s'attache à montrer cette étrange filiation: elle fait du mémoire de Gérando une sorte de contrepoint «moral » au mémoire «physique » et proprement dit an-

archives de la Société des observateurs de l'homme, on lui doit notamment Les Origines du Musée d'ethnographie, histoire et documents (Paris, Ernest Leroux, 1890 ; réimpression Jean-Michel Place, Paris, 1989) ainsi que de nombreux articles parus dans L'Anthropologie sur I'histoire du Muséum national d'Histoire naturelle. Sur Hamy, on peut consulter l'ouvrage très bien documenté de N. DIAS, Le Musée d'ethnographie du Trocadéro (1878-1908). Anthropologie et muséologie en France, Paris, CNRS, 1991.

32 Georges Hervé, membre comme E.-T. Hamy de la Société d'anthropologie de Paris, enseigna dans le cadre de celle-ci l'histoire de l'ethnologie. On lui doit de nombreux articles sur cette histoire et, surtout, l'édition de textes provenant de la Société des observateurs de l'homme.

33 M. BOUTEILLER, «La Société des observateurs de l'homme, ancêtre de la Société d'anthropologie de Paris», Bulletins et Mémoires de la Société d'anthropologie de Paris, 10e série, 1956, pp. 448-465. 
thropologique de Cuvier, sur lequel d'ailleurs elle insiste plus volontiers, - celui de Gérando n'ayant droit qu'à un commentaire rapide où [20] l'accent est singulièrement mis sur ses côtes «naturalistes», au détriment donc des considérations linguistiques, sociologiques, ethnologiques (au sens culturaliste du terme) qui forment les deux tiers du mémoire et en constituent certainement l'apport le plus original. Cette nette accentuation par Bouteiller des aspects anthropologiques et naturalistes des travaux de la Société des observateurs de l'homme traduit assez bien les tentatives d'appropriation et de récupération dont ces travaux ont été l'objet de la part de la Société d'anthropologie de Paris et explique peut-être leur méconnaissance, voire leur « rejet» par l'École française de sociologie. Une telle lecture va en tout cas dans le sens des remarques que Broca exposait déjà dans sa communication du 8 juillet 1869, par lesquelles d'ailleurs Bouteiller conclut son article. Broca ${ }^{34}$ y déplorait le fait que «les naturalistes [qui avaient selon lui fondé la Société des observateurs de l'homme] s'étaient trop hâtés de faire appel au concours des philosophes et des lettrés. L'anthropologie n'était pas encore assez solidement constituée pour retenir dans sa sphère les forces étrangères qu'elle avait apportées à son aide. Au lieu de les fixer sur son terrain, elle avait été entraînée à leur suite, sur le sol mouvant de la Politique». Que le mémoire de Gérando ait pu gêner les membres de la Société d'anthropologie, cela ne fait pas de doute: l'approche par les signes et par le langage préconisée par Gérando, ses recommandations de prendre en compte aussi bien, et avec autant d'importance, les systèmes de pensée et d'organisation sociale des peuples «sauvages» que leur morphologie, leur anatomie et leur environnement naturel, allaient certes à l'encontre du naturalisme, du positivisme anatomique volontiers affiché et défendu à l'époque.

Il faudra en fait attendre les études de Jean Poirier et celles de Gusdorf 35 pour que l'ethnologie française, I'anthropologie sociale et

34 P. BROCA, Histoire du progrès des études anthropologiques depuis la fondation de la Société, séance solennelle du 8 juillet 1869, Paris, Hennuyer, 1870.

35 Cf. J. POIRIER, « Histoire de la pensée ethnologique», in J. POIRIER (éd.), Ethnologie générale, Paris, Gallimard, 1968, pp. 26-27 ; J. POIRIER, op. cit., 
culturelle, « récupère » ce texte et l'intègre à son histoire, en fasse un repère archéologique. Mais là aussi, ce fut par un biais, celui de I'histoire en tant que discipline, que Gérando eut droit à la reconnaissance des ethnologues: s'il n'y avait eu les recherches de Jean-Paul Faivre, océaniste et, en particulier, l'un des premiers historiens de l'expédition du capitaine Baudin «aux terres australes»36, s'il n'y avait eu sa collaboration avec l'ethnologue Jean Poirier, peut-être que Les Considérations... reposeraient encore sur les rayonnages de la bibliothèque du Muséum national d'Histoire naturelle! Cette découverte, cependant, ne donna lieu à aucune analyse précise bien que l'on reconnût à Gérando le mérite d'avoir le premier parlé « d'observation participante » 37, non plus qu'à une [21] réédition du mémoire. Seuls les Anglo-saxons, bien plus attentifs aux « archives » de l'anthropologie, et préoccupés par son histoire que les Français ne le sont, en fournirent en 1969 une édition largement introduite par F.T.C. Moore ${ }^{38}$. À la décharge des auteurs français, tardivement intéressés comme on l'a vu par ce mémoire, il convient de signaler que - qu'elle qu'ait été la part novatrice des réflexions ou celle des intuitions pour ainsi dire géniales qui présidèrent à sa rédaction - il ne déboucha sur aucune fondation proprement dite de la discipline, l'auteur lui-même paraissant s'en être désintéressé dans la suite de sa carrière et de ses œuvres. À ce titre, on ne peut parler à son propos de « texte précurseur » ni voir en de Gérando un « père fondateur ». Outre des raisons d'ordre épistémologique que nous exposerons plus loin et qui, à notre sens, expliquent le peu de cas que l'anthropologie française a

1969, pp. 21-23; G. GUSDORF, «Ethnologie et métaphysique: l'unité des sciences humaines », in J. POIRIER (éd.), op. cit., 1968, pp. 1788-1789.

36 J.-P. FAIVRE, L'Expansion française dans le Pacifique, 1800-1842, Paris, Nouvelles éditions latines, 1953 ; cf. notamment «L'expédition Baudin et les velléités de la politique impériale », pp. 73-183.

37 C'est en ces termes que le présente le Petit Robert des noms propres : «Il a donné également un des premiers guides d'enquête ethnologique, à l'occasion de la mission de découverte en Terres australes dirigée par Baudin; il y affirme, avant B. Malinowski, la règle de l'observateur participant. »

38 Cf. J.-M. DE GÉRANDO, The Observation of' Savage People, édité par F. T. C. MOORE, Berkeley, University of California Press, 1969; préface de E. E. EVANS-PRITCHARD. 
fait de ce mémoire, il existe au moins trois raisons circonstancielles qui, de fait, en ont limité la portée et ont sans doute empêché qu'il ait une filiation théorique directe.

La première - on l'a vu - est liée à l'existence même de la Société des observateurs de l'homme et au groupe des Idéologues dont les activités et l'influence politiques cessèrent dès la proclamation de l'Empire.

La deuxième raison tient au projet même du mémoire : conçues et rédigées, ainsi que le précise l'avertissement, à l'attention des expéditions scientifiques dirigées par Baudin et Levaillant dans le but de fournir un guide d'enquête aux savants les accompagnant, les Considérations... ne tiennent compte ni des conditions ni a fortiori des contraintes matérielles que suppose et implique ce type d'expéditions. Celles-ci, en partie vouées à la découverte proprement dite, à la reconnaissance, aux relevés géographiques des terres et des côtes, principalement orientées vers la collecte d'échantillons botaniques et zoologiques, motivées par la constitution ou l'enrichissement de séries muséographiques autant que par la prise de possession de territoires nouveaux, ces expéditions étaient essentiellement itinérantes, nous dirions aujourd'hui de nature plus extensive qu'intensive. De ce point de vue, elles ne pouvaient offrir de situations qui répondissent aux règles de méthode posées par Gérando comme préalables à toute étude ethnographique sérieuse et rigoureuse: l'apprentissage de la langue des «naturels», l'insertion dans la communauté étudiée, la participation aux événements quotidiens ou rituels, etc. Bref, ces directives supposent des haltes assez longues, un temps d'enquête et d'observation qui n'est pas celui d'une expédition de découvertes. On peut s'étonner que Gérando, faisant preuve par ailleurs d'une grande perspicacité, ait autant négligé ce facteur temps. Sans doute emporté par sa critique des précédentes expéditions et voyages scientifiques a-t-il voulu préserver celle de Baudin de ces mêmes critiques, prenant en quelque sorte les devants méthodologiques, proposant une sorte d'idéal de la situation d'enquête. Ceci montre les limites de l'observation telle que les Idéologues ont pu la concevoir: elle reste un lieu théorique. 
La troisième raison tient à son audience : peu de savants de l'expédition étaient en mesure de comprendre et de suivre les conseils de Gérando, pour l'excellente raison qu'il n'y avait pas d'ethnographes! Principalement composée de botanistes, de zoologistes, de géographes et de minéralogistes, l'équipe scientifique envisageait plus de prélever et collecter des échantillons et des spécimens, de dresser des cartes, de reconnaître des rivages, etc., que d'observer les mours et coutumes des peuples «sauvages». Seul François Péron recruté au dernier moment comme zoologiste et anthropologiste aurait pu s'en inspirer si sa personnalité, son ambition et sa formation avaient été différentes. Celles-ci compromirent pour une part la réussite du voyage, notamment par l'affrontement sourd mais constant qui eut lieu entre Baudin et lui.

Étudiant en médecine, élève de Cuvier, Péron se disait lui-même, avec une complaisante lucidité, " inconséquent, étourdi, disputeur, indiscret, trop entier dans [ses] opinions personnelles, incapable de céder jamais à aucune raison de convenance, [il pouvait se] faire des ennemis et aliéner [ses] meilleurs amis. »

«Ces défauts, ajoutait-il, sont la suite de mon éducation et de l'état d'indépendance dans lequel j'ai vécu. Je sais qu'ils ternissent les qualités que je puis avoir : mais telle est l'emprise de l'habitude, que mes efforts pour m'en corriger ont été inutiles jusqu'à ce jour. Cependant, en me les reprochant, je n'en rougis point. Je sens que mon cœur est étranger au mal que j'ai pu faire; et le regret que j'en ai m'excuse au tribunal de ma conscience. 39 » 
Rapidement formé avant son départ par Lacépède 40 , Gérando et Cuvier, Péron ne retint surtout que les instructions anthropologiques de ce dernier ${ }^{41}$. Il devait principalement orienter ses recherches vers la mesure de la force musculaire des « sauvages » qu'il rencontra au hasard des escales et, incidemment, vers la collecte d'objets ethnographiques. Au départ animé des meilleures intentions, tenant un discours humaniste émaillé de considérations rousseauistes sur la bonté, la « santé et la longévité des sauvages», Péron devait vite déchanter, et modérer ses enthousiasmes lors de ses premières rencontres avec les «sauvages»; puis rectifier ses jugements au point même de prendre le contre-pied des idées développées dans son mémoire de 1800. Les vicissitudes des rencontres, les difficultés de communiquer avec les populations autochtones de même que l'état de « débilité physique » et de dénuement [23] moral et intellectuel dans lequel il les «trouva - et qu'il décrivit amplement - le déçurent et lui firent remettre en question cette idée hasardée à l'époque que la perfection physique est en raison inverse de la perfection morale : le « sauvage » n'était ni beau ni bon! 42

40 Bernard Lacépède (1756-1825), ancien collaborateur de Buffon, fit des leçons sur l'anthropologie dans son cours professé au Muséum en 1800 et 1801 . Lors du discours d'ouverture du cours de zoologie de l'an IX, Lacépède mentionne l'existence de la Société des observateurs de l'homme: «Nous allons tâcher de répandre quelques lumières nouvelles sur un des objets les plus dignes de I'attention du naturaliste : nous allons nous occuper de I'homme... Quel moment plus favorable pour parvenir à ce but, que celui où les sciences prennent une direction plus particulière vers la connaissance de l'homme, où des naturalistes, des voyageurs, des philosophes de premier ordre, viennent de se consacrer à son observation (ils viennent de fonder la Société des observateurs de l'homme) ; et où la fameuse sentence des sages de la Grèce: "Connais-toi toi-même", est devenue l'honorable devise de leur illustre association. » Cours de zoologie, discours d'ouverture, Paris, Plasson, 1800.

41 M. GIRARD, op. cit., 1857, p. 21. Il remarque à ce propos que «Péron obtint avec reconnaissance une note détaillée de la main de cet illustre savant, indiquant les nombreux points sur lesquels devaient porter ses investigations. Il attachait tant d'importance à ce précieux écrit qu'il ne passait pas un jour sans le relire. »

42 On attribue généralement à Montaigne l'invention du mythe du «bon sauvage » (voir le chapitre «Des Cannibales » des Essais). Ce mythe fut précisé et enrichi au début du XVIIIe siècle par les relations de L.-A. LAHONTAN (1666- 
Le commentaire que Péron écrivit à propos d'une planche gravée par Lesueur, dessinateur, avec Nicolas-Martin Petit, de l'expédition du capitaine Baudin, est à ce titre révélateur. Nous le livrons ici en entier, en raison même de ses difficultés d'accès (il s'agit d'un passage manuscrit, retiré de l'édition originale du Voyage de découvertes aux Terres australes, retrouvé par E.-T. Hamy à la bibliothèque du Muséum d'histoire naturelle du Havre ${ }^{43}$ ), mais aussi et surtout parce qu'il nous semble être représentatif de la démarche inaugurée par Pé-

1715), Dialogues de Monsieur le baron de Lahontan et d'un Sauvage de l'Amérique, Amsterdam, De Boeteman, 1740 (Paris, Éditions sociales, 1970). Sur ce problème, et parmi les rares études en français consacrées à l'histoire ou à la protohistoire de l'anthropologie, on peut notamment consulter l'article de $G$. HERVÉ, «Les débuts de l'ethnographie au XVIIIe siècle (1701-1765) », Revue de l'école d'anthropologie, 1909, pp. 345-366, et pp. 381-401; le chapitre de VAN GENNEP, op. cit., 1914, pp. 94-218; I'importante et remarquable étude de C. MINGUET, Alexandre de Humboldt, Paris, Maspéro, 1969, pp. 323-355, où il est question des représentations divergentes, voire contradictoires, du sauvage à la fin du XVIIIe siècle : I'article un peu rapide de H. CLASTRES, « Sauvages et civilisés au XVIIIe siècle», in F. CHATELET (éd.), Histoire des idéologies, t. III, Paris, Hachette, 1978, pp. 209-228, où référence est faite aux Considérations... de Gérando (pp. 226-228) ; ainsi que la thèse de M. DUCHET, Anthropologie et histoire au siècle des Lumières, parue chez Maspéro en 1971 et rééditée chez Flammarion en 1977, curieusement allégée de ses références érudites. Dans le chapitre consacré à l'anthropologie de Buffon, M. Duchet montre que l'idée du « bon sauvage » était déjà vivement contestée par celui$\mathrm{ci}$, qui, se fondant sur les relations de certains voyageurs, pouvait affirmer que «les sauvages du Nouveau Monde sont moins robustes, moins sensibles, plus craintifs et plus lâches que les Européens. Ils n'ont "nulle vivacité, nulle activité dans l'âme". "Ils manquent d'ardeur pour leur femelle" - le mot renvoie à la sexualité animale - "et par conséquent d'amour pour leur semblable [...] ". Cette indifférence pour le sexe est la tache originelle qui flétrit la nature, qui l'empêche de s'épanouir, et qui, détruisant les germes de la vie, coupe en même temps la racine de la société. "À l'autre extrême, l'Européen vivant sous un climat tempéré et dans un pays policé représente la perfection du type. » ( $p$. 97). On verra que Péron reprendra à son compte cette vision des sauvages, mais il cherchera à la fonder sur des observations et des mesures, et ne la jugera pas définitive : les sauvages, pour lui et à la différence de Buffon, ne sont pas des « dégénérés » mais des « ratés » de la civilisation qu'une « éducation appropriée » peut donc corriger.

43 Cf. E.-T. HAMY, «L'œuvre ethnographique de Nicolas-Martin Petit, dessinateur à bord du Géographe (1801 - 1804)», L'Anthropologie, T. II, 1891, pp. 601-622. 
ron. En effet, se trouve ici marquée une rupture, un renversement de perspectives dans l'ordre des représentations du « sauvage» qui, bien que toujours perçu comme un primitif, comme un homme de la nature, n'est plus valorisé par cette qualité, n'est plus gratifié d'une perfection ou d'une puissance physiques que l'on voulait alors déduire de cet état de nature. Péron soutient au contraire que la trop grande proximité du «sauvage » avec l'ordre de la nature, affaiblit son corps et altère son esprit. Il en a pour preuve ses propres observations, certes rapides et quelque peu fantaisistes portant sur la sexualité des « sauvages », par conséquent sur des comportements considérés comme [24] entièrement gouvernés par les « lois naturelles». E.-T. Hamy relate ainsi les conditions dans lesquelles se déroulèrent les observations de Péron: «Péron et Petit, accompagnés d'un maître d'équipage et de deux matelots, sont descendus dans l'île Maria, et quatorze naturels les entourent, leur palpent les mollets, la poitrine, et veulent s'assurer qu'ils ont bien, au milieu d'eux, des hommes blancs, il est vrai, mais conformés comme ils le sont eux-mêmes. Ils insistent surtout auprès d'un des matelots, le citoyen Michel, comme le nomme Péron, jeune et imberbe, et que notre naturaliste prie de se rendre à leurs sollicitations ». Péron décrit alors la scène:

« Michel exhiba tout à coup des preuves si frappantes de sa virilité, que tous à la fois poussèrent de grands cris de surprise mêlés de grands éclats de rire qui se répétèrent à plusieurs reprises. Cet état de force et de vigueur dans celui d'entre nous qui en paraissait le moins susceptible les surprit extraordinairement, ils avaient l'air d'applaudir à cet état, comme des gens auxquels il ne serait pas très ordinaire. Plusieurs montraient avec une espèce de dédain leurs organes mous et flasques, ils les agitaient vivement avec une expression de regret et de désir qui semblerait indiquer qu'ils ne l'éprouvent pas aussi fréquemment que nous ${ }^{44}$. Sans doute, il serait indiscret d'affirmer sur

44 C'est ce geste qu'aurait reproduit Lesueur sur la planche en question : il donna lieu à des interprétations contradictoires de la part de A. de Quatrefages et de E.-T. Hamy. Ce dernier s'en explique dans son article (p. 611) : « Ce personnage, appuyé sur sa sagaie, se touche le pénis avec un rictus tout spécial, qui 
de simples apparences la réalité d'une observation aussi importante. Mais je ne crois pas devoir négliger de l'indiquer ici, en me proposant de ne rien oublier dans la suite pour approfondir cet objet: je dois même ajouter dès à présent que parmi le nombre assez considérable de naturels que j'ai vus jusqu'à ce jour, je n'en ai pu trouver aucun encore dans cet état assez fréquent chez l'homme civilisé, alors surtout qu'il est à la fois jeune, sain et vigoureux.

Comme dans la plupart des animaux, poursuit Péron toujours à propos des Tasmaniens, n'éprouveraient-ils le besoin de l'amour qu'à des époques déterminées et périodiques? La continuité des désirs et conséquemment aussi celle des jouissances seraient-elles donc un des bienfaits de la civilisation? Sans doute, il ne faudrait pas se décider trop légèrement à cet égard, la question est trop importante, elle est aussi trop délicate: cependant, si nous faisons attention à l'influence toute puissante des circonstances physiques sur la naissance des désirs, sur leur exaspération, leur continuité, il sera, je crois, très difficile de ne pas tomber d'accord avec moi, sinon sur la périodicité des désirs et des besoins de l'amour dans l'homme qui nous occupe, du moins sur leur rareté, sur leurs longues interruptions. En effet, si l'on calcule l'influence réunie, et de la température toujours assez forte dans laquelle nous vivons, et de l'abondance de nos aliments et de leurs qualités, et celle des assaisonnements, des liqueurs fortes dont nous faisons usage,

ouvre largement sa bouche et montre des dents grosses et bien rangées. Ce jeu de physionomie, assez mal rendu dans la planche, et le geste qu'il commente et souligne ont été très diversement appréciés. J'avais cru, pour ma part, y reconnaître l'expression d'une insulte cynique, dont il ne serait pas bien difficile de trouver l'équivalent dans les basses classes de nos grandes villes. M. de Quatrefages y avait vu tout autre chose. Un des hommes debout, disait-il en décrivant la planche de Lesueur, "ramène avec soin son prépuce sur le gland, que ce repli cutané est destiné à recouvrir". C'était à ses yeux, une notion de "pudeur masculine" qui se traduisait d'ailleurs à peu près de la même manière chez certains peuples polynésiens. "Cf. A. DE QUATREFAGES, Hommes fossiles et hommes sauvages. Études d'anthropologie, Paris, Baillère, 1884, p. 344 (réimpression Jean-Michel Place, Paris, 1988). 
et celle de l'oisiveté que bien souvent nous éprouvons; et celle de l'exemple, puissante sur les cœurs, et celle de notre éducation, de nos lectures, de nos parures, de nos ornements, de nos exercices, de nos réunions en société, etc., etc., l'on concevra bientôt que tout dans I'homme [25] civilisé se réunit pour faire naître le désir, pour le soutenir et le rallumer sans cesse à toutes les époques de l'année et dans presque toutes les circonstances de la vie.

Au contraire, errant au milieu des bois et des forêts, sans vêtements, sans asile, exposé perpétuellement aux intempéries d'une atmosphère humide et froide, manquant souvent des substances nécessaires à la vie, étranger à toute espèce d'assaisonnements ou de liqueur spiritueuse, connaissant à peine le repos, bien loin d'être livré comme le riche opulent aux langueurs de l'oisiveté, l'homme de la nature ne se trouve-t-il pas placé dans une position telle, que tout concourt à modérer la vivacité de ses désirs, à les amortir, à les éteindre promptement au milieu des rigueurs de l'hiver et quelquefois aussi des anxiétés de la famille? Doit-il conserver cette vigueur que par cent moyens étrangers à notre nature nous savons ranimer et soutenir, en maîtrisant toutes les circonstances physiques qui doivent la détruire chez l'homme qui nous occupe. Mais c'est assez et trop longtemps peut-être insister sur cet objet, que dans la suite de cette expédition j'aurai sans doute l'occasion d'approfondir davantage. Il me suffit d'indiquer maintenant qu'il n'est pas tout à fait invraisemblable que le sentiment de l'amour et le besoin de le satisfaire ne soit dans l'homme de la nature, sinon périodique, comme dans les animaux, du moins beaucoup plus rare et moins longtemps soutenu qu'il ne l'est dans l'homme réuni en société : d'où il résulte que la continuité des désirs et celle des jouissances de l'amour pourraient bien être le produit de la civilisation, et certes ce ne serait pas le moindre de ses bienfaits, que cette vivacité toujours renaissante de sensations douces et voluptueuses, source féconde des sentiments les plus vifs, les plus délicats et les plus chers. » 
En s'interrogeant sur la sexualité des «sauvages», sur leurs désirs et leurs jouissances, cela à partir d'un événement pour le moins inattendu, Péron entendait donc montrer que la dépendance des primitifs au milieu naturel pouvait limiter les expressions et les manifestations de leur sexualité ; que cette dépendance, au contraire des idées qui étaient souvent avancées au sujet de cette «activité » humaine mais naturelle sur laquelle elle aurait dû avoir une influence positive, «bénéfique », bridait ce que l'on appelle d'ailleurs I'instinct sexuel.

En attribuant à l'état de civilisation - mais de civilisation occidentale - le développement, la permanence et la sublimation de cet instinct, Péron renversait la représentation dix-huitièmiste du « sauvage », remettait en cause l'image idéale de la nature et de l'homme naturel, témoignait certes d'un ethnocentrisme que Gérando avait pourtant sévèrement critiqué, et justifiait du même coup les notions de progrès et d'évolution. Sur ces points, Péron n'innovait pas. Ces thèses étaient «dans l'air » à la fin du XVIIIe siècle : en 1794, Condorcet avait formulé l'idée de progrès et avancé celle de la «perfectibilité indéfinie de l'homme » 45 ; Volney avait, lors de son voyage forcé aux États-Unis, critiqué Rousseau et contesté son tableau du « bon sauvage», dressé, écrit-il, d'après « des comparaisons tirées de la forêt de Montmorency $\gg 46$; les relations des voyages de Cook, Forster, Bougainville et Lapérouse avaient par ailleurs sérieusement égratigné le mythe. Comme le note Charles [26] Minguet 47, cette fin du XVIIIe siècle voit « un remarquable déplacement géographique de la terre d'élection, du paradis perdu de l'homme naturel. Cette translation dans l'espace, depuis l'Amérique jusqu'aux îles du Pacifique, est la dernière étape, relativement courte, avant l'extinction définitive du mythe. »

45 Cf. M.-J. CONDORCET, Esquisse d'un tableau historique des progrès de l'esprit humain, Paris, Agasse, 1794. [Livre disponible dans Les Classiques des sciences sociales, JMT.]

46 Cf. C.-F. VOLNEY, Tableau du climat et du sol des États-Unis, Paris, Firmin Didot, 1846 (Ire édition 1803) ; voir aussi C. MINGUET, op. cit., 1969, p. 332.

47 C. MINGUET, op. cit., 1969, p. 333. 
En conclusion de son étude sur les Considérations... de Gérando, Stocking 48 évoque le déclin de l'idée du bon sauvage. La dégradation des conditions de vie des « sauvages " constatée empiriquement dès la fin du XVIIIe siècle (par Péron notamment) finit par ne plus être considérée comme le résultat d'intrusions extérieures mais comme un état sul generis, propre à des différences de nature et de race. Gérando croit encore à la possibilité d'idées abstraites chez les « sauvages »; pour Herbert Spencer, soixante ans plus tard, il n'en sera absolument plus question. De même E. B. Tylor qui se réclame d'un certain XVIIIe siècle, inverse la visée de Gérando: «Pour les deux hommes, la science de l'anthropologie était essentiellement la "science d'un réformateur". Mais le but de leur réforme n'était pas le même. Pour Gérando, il s'agissait d'élever les peuples sauvages: pour Tylor, c'était pour supprimer les derniers restes de sauvagerie et de barbarie de la société civile d'Europe. »

À ces deux images contradictoires du "sauvage " - d'un côté celle des rêveurs, des poètes et des philosophes des Lumières, de l'autre celle des voyageurs, déçus ou perspicaces, confrontés à la dure réalité des échanges et des relations avec ces peuples lointains - Péron substituera une autre image: celle du « sauvage comme objet d'étude, sujet d'expérience, à la limite comme échantillon, témoin d'une autre nature humaine. Partant de la déconvenue et de la déception, toutes subjectives, des voyageurs philosophes, tel Volney, ou des siennes propres, Péron tenta de les fonder, de les justifier, de les expliquer par l'étude voulue rigoureuse et objective de l'anatomie ou de la condition physique des « sauvages», de ce qui était par conséquent le plus visible et le moins susceptible d'être remis en question par les nostalgiques d'un âge d'or ou d'un paradis perdu, lesquels se voyaient de la sorte "scientifiquement» rejetés du côté de l'imagination ou de l'idéologie (au sens moderne du terme):

48 G.W. STOCKING, art. cit., 1964, pp. 140-150. 
«Elle est récente encore, écrira-t-il 49 , cette époque singulière où l'on vit des hommes célèbres, entraînés par une imagination ardente, aigris par les malheurs inséparables de notre état social, s'élever contre lui, en méconnaître les bienfaits, et réserver pour l'homme sauvage toutes les sources du bonheur, tous les principes de la vertu. Leur funeste éloquence égara l'opinion; et, pour la première fois, on vit des hommes sensés gémir sur les progrès de la civilisation, et soupirer après cet état misérable, illustré de nos [27] jours sous le nom séducteur d'état de nature... Heureusement les voyageurs modernes, en nous faisant successivement connaître tant de peuples sauvages, nous ont permis d'apprécier ces vains sophismes à leur juste valeur ; et notre expédition, sous ce rapport, aura pu servir utilement la vraie philosophie. »

Les autres investigations « ethnographiques» de Péron allèrent dans le même sens, et contribuèrent peut-être, tant sur le plan des techniques d'enquête que sur celui des analyses et des interprétations, à engager l'anthropologie dans une problématique biologique (I'anthropologie physique), voire médicale 50 . Les mesures dynamométriques auxquelles Péron accordait une valeur méthodologique et démonstrative sans égale, et dont l'utilisation lui fut inspirée par la lecture des travaux de Charles-Augustin Coulomb 51, systématiquement effectuées auprès des populations rencontrées, lui « permirent» de confirmer ses hypothèses sur la fragilité des hommes de la nature. Comparées à celles obtenues auprès d'un échantillon européen, elles

49 F. PÉRON, «Esquisse sur la force physique des peuples sauvages de la terre de Diémen, de la Nouvelle-Hollande, etc. », in F. PÉRON ET L.-C. FREYCINET, Voyage de découvertes aux terres australes, Paris, Imprimerie royale, 1816, pp. 446. (Texte reproduit in fine.)

50 Cf. J. JAMIN, « Anthropologie française», in P. BONTE \& M. IZARD, Dictionnaire de l'ethnologie et de l'anthropologie, Paris, P. U. F., 1991.

51 Et notamment par le mémoire que COULOMB lut à l'Institut en 1797 : De l'utilité de déterminer la quantité d'actions journalières que les hommes peuvent fournir par leur travail particulier suivant les différentes façons dont ils emploient leur force. 
traduisaient ce que Péron appela « la débilité musculaire des sauvages », imputable selon lui aux conditions climatiques, aux régimes alimentaires, à l'alternance continuelle de disette et d'abondance, aux fatigues provoquées par la recherche de nourriture, etc., - conséquences de "l'état sauvage dans lequel végétaient ces malheureuses peuplades 52 . $\gg$ Armé donc du seul dynamomètre de Régnier 53 , Péron pouvait conclure que «le perfectionnement de l'état social, en rendant I'homme des contrées sauvages à l'abondance, pourrait déterminer un développement considérable des forces physiques, et faire disparaître les vices de sa conformation actuelle ${ }^{54}$. " Ces expériences et ces interprétations pouvaient dès lors justifier toute mission civilisatrice et toute colonisation...

Auteur et éditeur intellectuel du rapport scientifique de l'expédition, Péron contribua ainsi à vulgariser et à officialiser I'« approche anthropologique » qu'il inaugura « sur le terrain» : celle qui, plus tard et pendant longtemps, prétendit expliquer les différences sociales et culturelles par un déterminisme biologique et géographique, qui prétendit les expliquer par les seules mesures comparées des caractères anatomiques, par l'influence des climats ou par l'effet des régimes alimentaires, etc.. Louis-François Jauffret semble annoncer cette approche dans la première partie de son Introduction aux mémoires de la Société des observateurs de l'homme; mais il prend soin d'en pondérer les aspects mécanistes par un large développement sur la nécessité d'étudier conjointement la génération des idées, la formation et la structure du langage, d'observer et de tenir compte des faits sociaux et culturels.

[28]

Avec Péron et les expériences qu'il réalisa, on est donc bien loin des Considérations... de Gérando, qui voyait dans le langage, les signes, les symboles et les représentations, l'objet d'étude privilégié de la science de l'homme et faisait des sociétés sauvages le lieu où leur

\footnotetext{
52 M. GIRARD, op. cit., 1857, p. 88.

53 Cf. J. JAMIN, « Note sur le dynamomètre de Régnier », Gradhiva, n 1, 1986, pp. 17-21; et ici même, infra. p. 179.

54 F. PÉRON, art. cit., 1816.
} 
formation et leur articulation pouvaient s'appréhender le mieux (car le plus simplement), témoignant d'un empirisme raisonné proche de celui d'Alexandre de Humboldt et annonçant l'idée de Durkheim du «primitif comme laboratoire sociologique ».

Rendre Péron seul responsable du devenir scientifique de l'anthropologie française au XIXe siècle et du peu de retentissements qu'eut l'expédition du capitaine Baudin dans I'histoire de l'anthropologie sociale et culturelle serait sans doute abusif. Il fut toutefois considéré - en tant que premier anthropologiste de terrain 55 - comme un pionnier par les membres de la Société et de l'École d'anthropologie de Paris. Ceci eut pour résultat de laisser dans l'ombre pendant près de cent cinquante ans celui qui, si l'on en juge par les quelques extraits de sa correspondance avec Jussieu que nous livrons dans ce volume, lut plus attentivement et plus sérieusement, semble-t-il, les Considérations... de Gérando et les mémoires de Jauffret, Nicolas Baudin.

Correspondant de la Société des observateurs de l'homme depuis sa fondation en 1799, Baudin avait certainement eu plus que Péron, trop vite formé, recruté et embarqué, le temps et la possibilité de se familiariser avec les idées et les projets des Idéologues de l'an VIII. Le soin qu'il mit à recopier le mémoire de Jauffret sur la constitution d'un muséum anthropologique au début de son journal de bord, le souci qu'il manifesta de collecter à chaque escale, à chaque halte, à chaque exploration, des objets ethnographiques, suffisent à le prouver... Il semblait en outre estimé par les savants naturalistes du Muséum com-

55 Cf. G. HERVÉ, «Les premières armes de Péron», Revue anthropologique, vol. XXIII, 1913, pp. 1-16. Cf. aussi A. QUATREFAGES, op. cit., 1884, p. 296, qui n'hésite pas à reconnaître en Péron « un excellent observateur [qui] eut de nombreuses rencontres avec les Diéménois et sut en profiter», même si plus loin il pondère ses jugements à propos des mesures dynamométriques. MOORE (op. cit., 1969) et J. HONIGMAN (The Development of Anthropological Ideas, Homewood, Dorsey Press, 1976) ont souligné la légèreté, voire l'incompétence de Péron. Honigman écrit d'ailleurs : «C'est triste à dire, mais les thèmes et les conseils d'enquête [de Gérando] aidèrent peu l'expédition australienne [de Baudin]. L'ethnographe inepte qui était à bord ne mena que des observations superficielles et ignora totalement les instructions de Gérando comme celles des voyageurs plus expérimentés » (p. 82). Porter un tel jugement, a posteriori, est pour le moins surprenant et méconnaît gravement la situation épistémologique des Idéologues. 
me en témoigne ce rapport de la main d'Antoine-Laurent de Jussieu 56 :

«Le capitaine Baudin, jaloux de mériter comme eux [les grands voyageurs] l'estime publique, a sollicité une mission du même genre. Il avait auparavant navigué pendant plusieurs années sous les ordres d'une puissance étrangère [I'Autriche] et avait recueilli pour elle beaucoup de productions vivantes qui subsistent encore dans ses collections. Bientôt son goût pour les recherches d'histoire naturelle et ses travaux dans cette partie furent connus des naturalistes français qui engagèrent le gouvernement à l'envoyer aux Antilles pour en rapporter quatre végétaux manquant au Jardin des Plantes. Il partit pour ces îles en I'an IV avec des adjoints instruits et actifs, et après deux ans d'absence, après avoir surmonté divers obstacles, il ramena en France un vaisseau chargé de plantes vivantes au nombre de plus de 1500 individus, la plupart ou non connus, ou au moins n'ayant pas encore existé dans les jardins d'Europe. Une collection nombreuse de graines fraîches, de plantes desséchées ou herbiers, de bois étiquetés avec soin, d'animaux préparés pour les galeries d'histoire naturelle, ajoutait [29] encore à l'intérêt de ce voyage, et l'on reconnut que jamais aucun navigateur n'avait procuré dans une seule expédition autant d'objets réunis. Ce premier essai devait inspirer aux amis de la science un nouveau désir de voir employer le capitaine Baudin à la tête d'une entreprise plus étendue, Les professeurs du Muséum d'histoire naturelle firent plusieurs tentatives auprès du Directoire pour déterminer un voyage dans les régions éloignées, moins connues et plus fertiles en objets absolument nouveaux. Des circonstances politiques ne permirent pas alors d'accueillir cette démarche. Baudin, que les obstacles ne décourageaient pas, communiqua en I'an VIII ses vues aux trois classes de

56 A.-L. Jussieu, Rapport sur le voyage entrepris par les ordres du gouvernement et sous la direction de I'Institut par le capitaine Baudin, 5 nivôse an IX. (manuscrit de 4 feuillets conservé à la bibliothèque du Muséum nation d'histoire naturelle sous la cote 1214.6). 
I'Institut dans l'espoir qu'un rapport avantageux de ce corps engagerait le nouveau gouvernement ami des sciences à approuver son plan et à en ordonner l'exécution. Il proposait un voyage complet autour du monde ayant pour objet des recherches de géographie ou d'histoire naturelle, pour ajouter aux grands navigateurs qui depuis quarante ans ont singulièrement agrandi le domaine de ces deux sciences. Trois vaisseaux devaient partir de conserve suivant le projet et revenir ensuite séparément de différents points et à différentes époques à mesure qu'on avait complété leur chargement en objets relatifs aux sciences et aux arts et surtout en végétaux et animaux vivants dont la naturalisation en France pouvait devenir pour la nation une nouvelle forme de prospérité. Les commissaires nommés par les trois classes pour leur rendre compte du mémoire du capitaine Baudin étaient les citoyens Bougainville, Fleurieu, Duteil, Camet, Lapla$\mathrm{ce}$, Lelièvre, Lacépède et Jussieu. Ils se réunirent pour examiner le plan proposé et bientôt leurs bases furent arrêtées. Un voyage autour du monde fut jugé inutile après les expéditions célèbres de ces navigateurs hardis qui avaient parcouru le grand océan sous divers parallèles... »

Mort en 1803 à I'Île Maurice, avant le retour de l'expédition en France, Baudin ne put témoigner ni pour lui-même ni pour les Idéologues et naturalistes qui avaient conçu ce qui demeure, malgré tout, la première expédition ethnographique de l'histoire des sciences de I'homme 57.

57 Les travaux de J.-P. Faivre, la publication du Journal de Baudin, récemment le colloque «Les Français et I'Australie» (Le Havre 1989) ont permis de mieux cerner la personnalité du chef de l'expédition, vivement égratignée par le rapport de Péron et les hagiographes de celui-ci ; cf., en particulier, J.-P. FAIVRE, «Les Idéologues de l'an VIII et le voyage de Nicolas Baudin en Australie (1800-1804) », Australian Journal qf French Studies, vol. III, $n^{\circ} 1,1966$, pp. 315 ; ainsi que The Journal of Post Captain Baudin, Adélaïde, Libraries Board of South Australia, 1974, (préface de J.-P. FAIVRE). 
Mais aux causes événementielles qui viennent d'être évoquées, s'ajoutent des causes plus profondes - théoriques et épistémologiques - qui expliqueraient en fin de compte l'oubli dans lequel les mémoires de la Société des observateurs de l'homme sont tombés. L'impasse faite par les principaux théoriciens du discours ethnologique français est suffisamment importante pour qu'au-delà de la simple méconnaissance ce soit le discours lui-même ou bien leurs propres démarches qu'il importe ici d'interroger. L'insistance avec laquelle les fondateurs institutionnels de l'anthropologie française (Durkheim, Mauss, LévyBruhl, Rivet, Lévi-Strauss, etc.) ont recherché l'origine et les fondements d'une science dans une épistémè (celle du XVIIIe siècle) semble révélatrice de cette symptomatologie philosophique, de cet hyperthéoricisme qui l'a longtemps caractérisée, et qui la rendent si peu sensible aux essais que les Idéologues firent au sujet des conditions d'observations des (autres) sociétés humaines.

[30]

On peut même penser que la naissance tout à fait tardive de l'ethnologie française de terrain (en gros après la Première Guerre mondiale) a transformé la période des origines en une espèce de paradis perdu, celui de l'Esprit des Lumières où cohabitaient la raison critique et le roman philosophique. L'absence de filiation lisible au cours du long $X I X e$ siècle «positiviste » a mis en branle le mécanisme de l'invention de la tradition. En Grande-Bretagne et aux États-Unis, l'expérience critique de l'évolutionnisme et du diffusionnisme débouche sur le fonctionnalisme et le culturalisme: les polémiques scientifiques, les innovations méthodologiques marquent une paradoxale continuité ignorée en France. En fait, c'est la modernité même des Idéologues qui les a desservis. Le silence sur la Société des observateurs résulte d'un point de vue qui fait coïncider la construction de l'objet ethnologique avec le moment de la construction historique de la discipline, alors qu'un bon siècle les sépare.

\section{Les silences de la méthode}


À lire les ouvrages d'histoire consacrés à l'ethnologie et l'anthropologie, on s'aperçoit immédiatement que cette histoire n'est qu'une histoire des idées théoriques, rarement celle des institutions, des enquêtes ou des terrains. Même lorsqu'il s'agit des problèmes de l'enquête, de la collecte des données, c'est une histoire qui porte sur les idées de méthode plutôt que sur la pratique et les conditions de possibilité d'exercice de cette pratique. Or nous sommes confrontés ici, avec les textes de Gérando et de la Société des observateurs de I'homme, à des propositions portant sur tous les domaines thématiques de l'anthropologie et cela dans une perspective méthodologique d'autant plus explicite qu'elle est nouvelle et volontaire. Volontaire, en effet, puisqu'une expédition devait mettre en application ces suggestions et ces orientations. Qu'elle ne l'ait pas fait est une autre histoire, comme nous l'avons vu, laquelle ne permet pas d'emblée d'assigner un statut précis à ces textes. Le silence prolongé sur les Considérations... de Gérando est d'autant plus étrange à première vue qu'il ne s'agit pas d'un texte isolé : il fait partie d'un ensemble idéologique et institutionnel, théorique et pratique, qui l'explique rationnellement même si a posteriori il peut apparaître comme un texte précurseur et comme un éclair de génie. Mais les textes précurseurs ne le deviennent que par la filiation qu'ils engendrent.

De ce point de vue, le silence sur de Gérando n'est pas dû à un hasard puisqu'il correspond à un silence constant sur les problèmes de méthode de terrain au sein de l'anthropologie française. Comment les armchair anthropologists, qui, en France, ont fondé et, pendant longtemps, dominé l'anthropologie auraient-ils signalé la rupture que peut introduire l'idée d'une observation participante anti-ethnocentrique, ceci cent quinze ans avant l'expérience de Malinowski (encore que ce dernier $n$ 'ait théorisé sa pratique qu'a posteriori) 58 ? Mais cette rupture ne va pas sans ambiguiités.

[31]

58 Comme le notent P. J. Pelto et G. H. Pelto: «... Le type de travail de terrain qu'il [Gérando] préconisait $n$ 'est devenu une réalité significative qu'au cours du XXe siècle. »; Cf. P. J. PELTO \& G. H. PELTO, « Ethnography: the Fieldwork enterprise », in J. HONIGMAN, (ed.), Handbook of Social and Cultural Anthropology, Chicago, Rand Mc Nally, 1973, p. 242. 
Avant de poursuivre, nous voudrions esquisser la logique de l'apparition et du développement de l'anthropologie en tant que telle. Seule une telle perspective peut nous permettre de définir la place de la réflexion méthodologique a prioriet d'apprécier par conséquent la signification des textes de Gérando et de Jauffret.

L'anthropologie existe lorsqu' elle a une pratique de terrain spécifique centrée sur les relations sociales. Cette pratique peut exister sans théorie explicite de méthode. La référence constante au XVIIIe siècle indique qu'il existe depuis longtemps une réflexion idéologique et théorique indirecte sur la nature des relations sociales et sur la façon de les appréhender. La question de méthode se pose progressivement: il faut systématiser la collecte des données et l'orienter, d'où une définition des idées directrices et des faits à recueillir. Une fois le principe de systématisation élaboré et les principes de critique des méthodes passées mis en pratique, nous disposons des prolégomènes à une méthode de l'anthropologie. Une histoire rapide nous prouve d'ailleurs que l'anthropologie pourra se faire sans méthode, c'est-à-dire sans utiliser celles qui sont disponibles 59 , ou en produisant la réflexion méthodologique a posteriori.

La possibilité matérielle d'accès au terrain « exotique », la présence d'institutions et de corps spécialisés dans le maintien de l'ordre politique qui déchargent le voyageur des contraintes à assurer sa propre sécurité, sont évidemment décisives. Mais rien n'empêche de penser avant cette phase typiquement coloniale la possibilité du contact et de ses effets sur la réflexion anthropologique. Nous pouvons voir sur ce point une rupture. J.-J. Rousseau l'a évoquée à plusieurs reprises et Gérando, sans en être son fils spirituel, en a systématisé le raisonnement dans la perspective explicite d'une enquête réelle. Évidemment surgit ici la dialectique de l'extériorité et de l'objectivité qui est au coeur de l'anthropologie. Comme l'explique Gérard Leclerc 60 : «... la compréhension de systèmes sociaux réels suppose une certaine extériorité par rapport à ce système. Seul un élément extérieur à ce dernier peut saisir la structure totale de ce système. C'est dire que

59 Ce qui est déjà le cas de Péron avec les Considérations... de Gérando.

60 Cf. G. LECLERC, Anthropologie et colonialisme, Paris, Fayard, 1972. 
l'anthropologue de terrain voit encore sa situation concrète d'Européen comme condition du savoir qu'il vise à élaborer 61 ». En fait, seules la situation coloniale et l'idéologie évolutionniste et victorienne permettent de fonder une position de savoir ethnocentrique et donc «objective ». Une fois constituée, il était possible de la remettre en cause, de la critiquer comme expression d'un rapport de domination et d'objectivation. Il serait donc tout à fait abusif de chercher dans une situation anticoloniale ou précoloniale (mais qui ne semble qu'attendre le moment propice pour se transformer) [32] la logique de l'anticolonialisme et donc des variétés de l'anti-ethnocentrisme contemporain. Nous en avons d'ailleurs pour preuve les péripéties pour le moins complexes du mouvement anti-esclavagiste contre la traite et pour l'abolition de la condition servile au cours de la Révolution française 62 . Cette lecture «pratique » de l'exotisme et de ses populations s'est également terminé par une intervention de Bonaparte qui réintroduit l'esclavage le 17 mai 1802 . Il y a là un parallèle pour le moins paradoxal avec la suppression de fait de la Société des observateurs de l'homme en 1803.

De fait, idéologie et méthode sont ici inséparables: antiethnocentrisme et observation participante sont les deux aspects d'une même logique de l'identification. Le silence ultérieur sur la méthode provient non seulement des changements idéologiques mais du refus d'en considérer les implications méthodologiques. L'hyperthéoricisme d'une part, le muséographisme empirique 63 d'autre part n'ont pas permis de poser la question de méthode. Il faut donc aborder celle-ci dans une perspective historique. Les instructions, guides ou méthodes d'enquête sont de nature variée et ont rempli des fonctions contradictoires. La littérature qui fait le point sur cet aspect de l'an-

61 G. LECLERC, op. cit., 1972, p. 94.

62 Cf. J. COPANS, The French Revolution and the Black People of and from Africa, Travaux et documents, $n^{\circ} 4$, Nairobi, Credu, janvier 1990. La bibliographie sur ce problème tout à fait symbolique est des plus succinctes dans I'historiographie de la Révolution française.

63 Le mémoire de Jauffret pose les bases d'une problématique dont nous attendons toujours les applications au niveau de la muséographie ethnologique. 
thropologie, la formalisation méthodologique et même théorique de l'enquête de terrain sont en réalité très minces. Ainsi, dans le chapitre consacré au travail de terrain dans le Handbook de Honigman 64, les auteurs, méthodologistes confirmés, consacrent $5 \%$ de leur texte à I'histoire de l'enquête et une des trois pages est consacrée à... Gérando. C'est pourquoi l'essai bibliographique de D. Fowler sur les «Instructions d'enquête 65 » est des plus utiles. Fowler nous présente sommairement plus de cent cinquante références d'instructions et guides en tous genres provenant d'Europe (y compris la Russie) et d'Amérique du Nord sur une période de quatre siècles. C'est dire l'importance de cette production d'où sont exclues les instructions très spécifiques (linguistiques, archéologiques) et toute la littérature professionnelle contemporaine d'après 1880 . Fowler classe cette littérature en quatre grandes catégories 66 :

«1. Les questionnaires élaborés par des organismes gouvernementaux pour recueillir des données sur les possessions coloniales ou sur des possessions éventuelles. Y sont inclus divers memoranda produits par le Conseil espagnol des Indes [33] entre 1577 et 1792 qui ont donné naissance a un grand ensemble de données utiles d'ordre ethno-historique, connu sous le nom de Relaciones geograficas 67 . Sont compris également des instructions spécifiques ou des manuels donnés à des équipes d'exploration patronnées par un gouvernement.

2. Des guides de voyageurs, des brochures ou des livres vendus commercialement comme guides pour des voyageurs cultivés dans des pays étrangers.

J. HONIGMAN, op. cit., 1973, pp. 241-288.

65 D. FOWLER, « Notes on Inquiries in Anthropology: a bibliographical Essay », in T. H. H. THORESEN (ed.), Towards a Science of Man. Essays in the History of Anthropology, Mouton, La Haye, 1975, pp. 15-32.

D. FOWLER, art. cit., 1975, p. 19.

67 H. CLINE \& AL., « The Relaciones geograficas of the Spanish Indies and New Spain, 1577-1792 », in Guide to Ethnohistorical Sources, part 1, Handbook qf Middle American Indians, 12, pp. 183-449. 
3. Des questionnaires élaborés par des sociétés savantes, que ce soit pour recueillir des données d'ordre général ou par pays précis. Les sociétés savantes ont souvent élaboré ces questionnaires pour des expéditions officielles.

4. Des questionnaires mis au point par des chercheurs individuels pour guider leur propre recherche 68 ou confiés à des personnes précises qui puissent leur procurer des informations sur des sujets définis. La plupart de ces travaux étaient des guides d'enquête de terrain, mais on pourrait y inclure des guides de recherche bibliographique comme celui de Herbert Spencer 69 qui servit à accumuler les données pour sa Sociologie descriptive (1873-1811). On considère habituellement le guide de Spencer comme le précurseur de Outline of Cultural materials de G. P. Murdock 70 et des Human Relations Area Files. »

Cette typologie des questionnaires et instructions d'enquête préprofessionnels nous montre bien que l'aspect « politique», pour ne pas dire « officiel», de ces préoccupations scientifiques est prédominant jusqu'au milieu du XIXe siècle. Ce qui paraît non moins évident c'est que les textes produits par la Société des observateurs de l'homme ne sont pas nouveaux en leur genre. Fowler note d'ailleurs « qu'à la fin du XVIIIe siècle il s'était développé en Europe une habitude de produire des questionnaires sur une diversité de sujets et sous une forme très variée. Aucun n'était strictement "anthropologique" et ils faisaient

68 Cf. J. G. BOURKE, Memoranda for Use in obtaining Information concerning Indian Tribes, Fort Omaha, Nebraska, privatery printed (reprinted 1964 in The Diaries of John Gregory Bourke, by E. V. SUTHERLAND, pp. 173-196); W. PETTY, Quaeries concerning the Nature of the Natives of Pennsilvania (reprinted 1927 in The Petty Papers, some unpublished Writings of Sir William Petty, edited by the MARQUIS OF LANDSDOWNE, Londres, Constable, pp. 115-118).

69 H. SPENCER, An Autobiography, New York, Appelton, 1904, p. 202 (vol. II).

70 Behavior Science Outlines, 1, 1965. 
partie d'une longue tradition de "compilation et de collecte" dans un grand nombre de domaines. » 71

Une brève comparaison entre la France, la Grande-Bretagne et les États-Unis ne sera pas inutile dans la mesure où nous sommes trop souvent portés à croire que la tendance française de l'évolution intellectuelle, idéologique et institutionnelle est typique. Or ici il n'en est rien. Dès la fin du XVIIIe siècle le terrain indien provoque aux ÉtatsUnis des enquêtes linguistiques et ethnographiques. Thomas Jefferson lui-même en a suggéré quelques-unes (du reste sous l'impulsion de Volney) et l'on [34] considère que le texte de Lewis Cass de 1823 constitue le premier manuel américain. De Cass on passe aux enquêtes de la Smithsonian Institution (dont celle de L.H. Morgan entre autres) et à la fondation par John Wesley Powell du Bureau d'ethnologie 72. L'anthropologie américaine, par son caractère à la fois national et autochtone, précocement institutionnalisée et appliquée, donne une place considérable à la collecte et à l'instruction d'enquête. Les obligations pédagogiques du XXe siècle élèveront cette production au niveau d'une caractéristique spécifiquement américaine. En Grande-Bretagne, c'est l'aventure coloniale (ses dangers aussi bien que ses moyens ${ }^{73}$ ) qui provoquera une floraison méthodologique. L'importance de l'expansionnisme britannique, l'expérience des cultures indiennes assimilée dès le milieu du XIXe siècle, la systématisation des questionnaires qui débouche sur les fameuses Notes and Queries on Anthropology 74 , les

71 D. FOWLER, art. cit., 1975, p. 19.

72 Cf. L. CASS, Inquiries, respecting the History, Traditions, Languages, Manners, Customs, Religion, etc., of the Indian living within the United States, Detroit, Sheldon and Reed, 1823 ; L. H. MORGAN, Circular in Reference to the Degrees qf Relationship among Different Nations, Smithsonian Miscellaenous Collections, 2 (10), 1862 ; voir aussi les textes de GIBBS sur l'archéologie de 1861, de HENRY sur le même sujet de 1878 et de MASSON sur les tribus indiennes de 1875.

73 Cf. J. GRUBER, « Ethnographic Salvage and the Shaping of Anthropology », American Anthropologist, vol. LXXII, 1970, pp. 1289-1299.

74 Le titre exact est : Notes and Queries on Anthropology for Use of Travellers and Resident in Uncivilized Lands, Londres, Stanford, 1874. (La dernière partie du titre a sauté dans les dernières éditions : il y en a eu six jusqu'à présent : 1892, 1899, 1912, 1929, 1951.) 
théories ultérieures (après la Première Guerre mondiale) de l'anthropologie appliquée sont autant de signes du sérieux attaché traditionnellement à la collecte de l'information et aux méthodes. Quant à I'orientation française elle va se restreindre et se limiter, par la suite, à l'anthropologie au sens médical et physique du terme ${ }^{75}$. La préoccupation ethnosociologique des Lumières puis des Idéologues n'aura pas d'effet direct au niveau de l'anthropologie. Cet oubli est également un oubli méthodologique, et il faut attendre le premier tiers du XXe siècle pour voir apparaître des instructions ou des manuels aussi sophistiqués que ceux des Américains ou des Britanniques 76 .

L'institutionnalisation pédagogique tardive et limitée de l'anthropologie française aura également pour effet de laisser dans l'ombre la préoccupation méthodologique. Enfin, la maturation récente de cette discipline s'est marquée par une "surthéorisation » conceptuelle ou épistémologique qui a poursuivi sous une autre forme [35] cette opération de censure. Le peu d'intérêt porté à la Société des observateurs de l'homme (pour ne pas dire l'ignorance pure et simple) par les anthropologues français contraste avec l'attention qu'on lui a prêtée outre-Manche ou outre-Atlantique. Le silence méthodologique français et le silence sur ce silence ont pour résultat une confusion lamentable concernant l'appréciation du passé et des origines de l'ethnologie en France, le rôle des Lumières, des Idéologues et une certaine forme de «médicalisation» du regard anthropologique C'est la rançon d'avoir

75 Cf. le discours de P. BROCA de 1869 (op. cit., 1870). Il sera traduit dès 1871 en anglais dans le Journal of the Anthropological Institute of New York, 1, pp. 22-42.

76 On notera pourtant la similitude des dates françaises et britanniques concernant la production des questionnaires pré-professionnels :

- 1841, Société ethnologique de Paris: Instructions générales adressées aux voyageurs (mémoire 1).

- 1860-1873, puis 1883, Questionnaires de la Société d'anthropologie de Paris (dont le Questionnaire de sociologie et d'ethnographie).

- 1841, TH. HODGKIN ET AL., Queries respecting the Human Race, to be adressed to Travellers and Others, British Association for the Advancement of Science, Report II, pp. 332-339 (voir du même également, 1852, vol. XXII, pp. 243-252). 
été précoce sur le plan des idées et d'avoir été en retard au niveau de la pratique 77 .

77 Cf. nos articles: J. COPANS, «Les tendances de I'anthropologie française», in Questions à la sociologie, P. U. F., 1976, pp. 45-60; «A la recherche de la théorie perdue: marxisme et structuralisme dans l'anthropologie française», Anthropologie et sociétés, vol. 1, 3, 1977, pp. 137-158; J. JAMIN, « L'histoire de l'ethnologie est-elle une histoire comme les autres ? , Revue de synthèse, 3-4, 1988, pp. 469-483. 


\section{La place des Considérations... de Gérando}

\section{Retour à la table des matières}

Ce rappel peut nous servir d'introduction aux lectures possibles de Gérando. Alfred Métraux, A.C. Haddon, R.H. Lowie, Wilfred Mühlmann, Arnold Van Gennep, Paul Mercier l'ignorent purement et simplement 78 . T.K. Penniman l'évoque à peine ${ }^{79}$. Nous avons vu dans quel sens M. Bouteiller et P.J. Pelto et G.H. Pelto abordaient les Considérations... Quant à $F$. Voget 80 il reprend en une page les remarques du traducteur anglais F.T.C. Moore. Dans son travail sur I'Histoire de la pensée ethnologique, Poirier cite les aspects méthodologiques du texte de Gérando et les instructions muséographiques de Jauffret ${ }^{81}$. Mais c'est Gusdorf qui approfondit la conjoncture historique marquant l'apparition et la disparition des idées de la Société des observateurs de l'homme. Il montre bien, en effet, dans quel sens se fait la réaction aux Lumières et aux Idéologues: «Malheureusement, note-t-il, les Idéologues, maîtres à penser de la Révolution et esprits libéraux, devaient nécessairement devenir suspects aux yeux de Napoléon. Et la Restauration les traite en vaincus lorsqu'elle entreprend de constituer en France une orthodoxie métaphysique. Le spiritualisme de Victor Cousin méprise les sciences de l'homme et crée à leur encontre un préjugé dont les effets n'ont pas cessé de se faire sentir jusqu'à nos jours. » 82 C'est

78 Cf. A. C. HADDON, History of Anthropology, Londres, Watts, 1949; R. H. LOWIE, The History of Ethnological Theory, New York, Farrar and Rinehart, 1937, (traduction française: Paris, Payot, 1971): W. MÜHLMANN, Geschischte der Anthropologie, Bonn, Universitats-Verlag, 1948 ; A. VAN GENNEP, op. cit., 1914 ; A. MÉTRAUX, « Les précurseurs de l'ethnologie en France, du XVIe au XVIIIe siècle, Cahiers d'histoire mondiale, vol. VII, $n^{\circ} 3,1963$, pp. 721738 ; P. MERCIER, Histoire de I'anthropologie, Paris, P. U. F., 1966.

79 T.K. PENNIMAN, A Hundred Years of Anthropology, Londres, Duckworth, 1935.

80 F.W. VOGET, A History of Ethnology, Londres, Holt, Rinehart and Winston, 1975.

81 L POIRIER, op. cit., 1968, pp. 27-28. 
jours. » 82 C'est dans cette conjonction d'un idéalisme positiviste et empiriste avec une réaction politique et idéologique que gît le démembrement définitif de l'héritage du XVIIIe siècle.

[36]

L'étude à la fois la plus générale et la mieux documentée nous vient - nous l'avons signalé - de l'anthropologue américain G.W. Stocking. Il retrace la fondation de la Société (en insistant notamment sur le rôle de Jauffret) et tente de dégager la signification de l'expédition Baudin. Puis il analyse tour à tour les Considérations... de Gérando, les Instructions... de Cuvier et les Observations... de Péron. Enfin, il conclut sur la portée de cette «pré-anthropologie». Il va jusqu'à écrire à propos de Gérando: "Avec toute leur rigueur empirique, ses instructions considèrent comme évidentes une conception de la nature du changement social ainsi que la méthode suivie dans sa propre étude. Cela devait faire partie de l'héritage et des acquis de l'ethnologie sociale et évolutionniste à la fin du XIXe siècle 83. "Cette vision des choses ne débouche cependant pas sur une classification raciale, $n$ 'insiste pas plus sur les différences dues à l'hérédité que sur celles dues à l'environnement.

Mais c'est Moore qui, en assurant la présentation et la traduction du texte de Gérando en anglais, en a réactivé la portée dans l'anthropologie contemporaine. Evans-Pritchard, dans sa très brève préface va jusqu'à dire: «L'étude se lit comme si elle avait été écrite hier ${ }^{84}$. 》 Moore, quant à lui, présente surtout les tenants et les aboutissants de l'expédition Baudin 85 . Il note le paradoxe des Considérations.... celui sur lequel nous avons insisté plus haut, oppose la perspicacité de Baudin à la vantardise illusionniste de Péron. Mais Moore veut savoir pourquoi les idées de Gérando sont restées lettre morte, au-delà des ava-

82 G. GUSDORF, art. cit., 1968, pp. 1790-1791.

83 G.W. STOCKING, art. cit., 1964, p. 140. Stocking s'est fait connaître depuis par ses nombreux travaux sur l'histoire de l'anthropologie et, surtout, par le fait qu'outre-Atlantique il a hautement contribué à faire reconnaître ce domaine de recherche comme un domaine à part entière.

84 Cf. F. T. C. MOORE (ed.), op. cit., 1969, préface p. X.

85 Moore semble ignorer les travaux de J.-P. FAIVRE et, en particulier, sa thèse de 1953 (op. cit., 1953). 
tars de l'expédition Baudin. En fait, le déclin des Idéologues, les travaux essentiellement compilatoires de la Société des observateurs de I'homme, l'optimisme colonial avant la lettre de Jauffret, de Gérando ou même de Péron, sont les signes d'une «maladie de l'époque » 86.

Pour Moore, cette maladie réside dans l'idéal du savoir utile ${ }^{87}$. La conclusion des Considérations... n'a donc rien de décoratif : le déclin des projets coloniaux provoque la disparition de la science projetée, puisqu'elle n'est plus utile 88 . Et cette contradiction amène Moore à conclure très justement: «Il lui manquait la perspective de faire éclater les idées de son temps, et pourtant il avait eu le mérite de proposer une méthode d'enquête de terrain en anthropologie qui ne se ressentait pas des préjugés, même si par ailleurs il les partageait luimême ${ }^{89}$. $" G$. Leclerc aborde en annexe de son étude pionnière la signification des Considérations... de Gérando 90. [37] Malgré la pertinence de son interprétation, nous pensons qu'il sous-estime, autant que les autres commentateurs, l'ouverture méthodologique de ce texte. Il pense que «ce que les philosophes demandent aux voyageurs, c'est seulement le doublet empirique de la déduction. Gérando, qui pourtant recommande l'observation empirique systématique, écrit au sujet des sauvages, [en] laissant entendre qu'il connaît déjà ses résultats [...] ${ }^{91}$. »Il est certain que les penseurs des Lumières, tout comme les Idéologues, n'ont pas de concept de société. Mais «l'origine est conçue par le XVIIIe siècle comme l'authentique alors qu'elle sera conçue par le XIXe siècle comme le simple (le grossier) et l'inachevé ${ }^{22}$. L'anti-ethnocentrisme n'est pas une simple précaution à l'égard des autres. C'est aussi un anti-ethnocentrisme « intérieur»

86 Moore définit cette notion de la façon suivante: « Je dirai qu'il y a maladie de l'époque lorsqu'à l'époque en question un ensemble d'idées domine de telle façon que n'importe quelle tentative pour les mettre en pratique produit un conflit entre plusieurs idées et les autres » (op. cit., 1969, p. 51).

87 F.T.C. MOORE (ed.), op. cit., 1969, p. 52.

88 F.T.C. MOORE (ed.), op. cit., 1969, p. 56.

89 F.T.C. MOORE (ed.), op. cit., 1969, p. 58.

90 G. LECLERC, op. cit., 1972, pp. 217-242.

91 G. LECLERC, op. cit., 1972, p. 221.

92 G. LECLERC, op. cit., 1972, p. 223. 
qui se méfie en Europe des prêtres, des despotes et des superstitions. Pour Leclerc il ne peut y avoir à cette époque de discipline spécialisée et autonome puisque les déterminations du primitif sont les mêmes, en dernière instance, que celles du civilisé (en dernière instance). Toutes les sociétés ont même des intérêts communs: les Lumières et le commerce non seulement ne sont pas incompatibles mais ils sont même complémentaires. Si Gérando condamne certaines pratiques coloniales, il ne manque pas d'insister sur la valeur du développement matériel et spirituel des Lumières.

Cela dit, explications et méthodes n'ont rien de sociologique ni même d'empirique. Le psychologisme et l'artificialisme dominent la théorie tandis qu'au niveau de la méthode on confond recherche empirique et déduction intuitive: "Milieu homogène et immédiat de I'homme, les idées peuvent s'observer dans le comportement des sauvages actuels ou se reconstruire à partir d'une sorte de déduction transcendantale des facultés. On a vu chez ceux-la mêmes qui, comme Gérando, tentent de jeter les bases d'une recherche empirique sur le terrain, comment il ne s'agit en quelque sorte que de la vérification des résultats de la recherche intuitive; la recherche de terrain est presque superflue: elle $n$ 'est à la rigueur que stimulation de la réflexion ${ }^{93}$. »

Il est certain que, dans le fond, Leclerc a raison d'être prudent, qu'objectivation, ethnocentrisme et colonisation vont de pair. Il n'empêche que les germes théoriques d'une rupture méthodologique qui fait passer une anthropologie spéculative et compilatoire à une anthropologie empirique et critique existent bel et bien chez Gérando (et de manière plus générale dans toute une série d'autres textes de la Société des observateurs de l'homme) et que ce seul fait mérite la prise en considération la plus attentive.

Les Idéologues sont à la fois les derniers représentants XVIIIe siècle et les premiers représentants du XIXe. Il serait donc erroné de ne voir dans les textes que nous avons réunis dans ce volume qu'un des aspects de cette situation transitoire. Car c'est justement cette unité des contraires qui leur donne toute leur puissance: c'est parce qu'il 
est pensé dans des cadres contradictoires avec sa logique que le [38] siècle des Lumières débouche ici sur la question de méthode. Mais c'est parce que la méthode qui fonde l'observation est pensée, elle, dans des termes antipositivistes qu'elle est en porte-à-faux avec les pratiques qui s'ébauchent et qui vont imposer de fait une autre orientation. Ces textes, malgré leur portée révolutionnaire 94 , sont comme mort-nés.

Ce sont évidemment les éléments d'un nouveau Discours de la méthode: ils sont à la fois signes d'une idéologie, bases d'une méthode et propositions d'une théorie. Or ces trois contraintes entrent en contradiction les unes avec les autres et les pratiques scientifiques qui semblent continuer les intentions de ces textes ne permettent pas de trancher dans quel sens de l'histoire va aller chacun de ces éléments. Gérando systématise une espèce de critique du sens commun mais il ne discute pas un instant (tout comme Jauffret d'ailleurs) la soi-disant évidence du cannibalisme. Le musée de Jauffret est en un sens une véritable utopie qui ne verra le jour que presque un siècle et demi plus tard. Mais il souhaite que des spécimens humains puissent venir jusqu'en France et l'attention portée au Chinois Tchong-A-Sam comme à l'enfant sauvage de l'Aveyron confirme cet extrémisme des tendances naturalistes qui annoncent par ailleurs l'esprit des expositions coloniales ultérieures. Il ne faut pas rechercher dans ces textes ce qui ne s'y trouve pas. Mais l'anthropologue ne peut s'empêcher de relier par un fil imaginaire la diversité des formes pratiques de l'observation hu-

94 L'esprit des Lumières plus l'idéologie de la Révolution française plus la « machine » bonapartiste et napoléonienne. L'antimodernisme libéral de ces dernières années a cru bon remonter jusqu'à cette époque pour retrouver - et extirper - les racines du totalitarisme. Les Lumières ne seraient plus les « lumières » et le XVIIIe siècle serait plutôt la fin de la modernité que son début. Le «totalitarisme» rationaliste de cette époque a pourtant constitué les prémisses de l'observation et de la pensée du social. Un tel phénomène historique et intellectuel ne peut être remis en cause sans démagogie. Évidemment certains peuvent regretter que le social et le politique puissent se penser de façon autonome : c'est bien ce que le postmodernisme libéral ne cesse de nous répéter. 
maine in vivo. Ishi, le dernier Indien «libre»95, les familles canaques de l'exposition coloniale de 1931, l'expérience américaine «Bio-sphère $2 »$ en Arizona, et plus largement l'enfermement artificiel comme méthode d'observation anthropologique ou biologique rappellent que le musée est un instrument pervers par nature, ce qu'à sa manière avait très bien perçu Michel Leiris: "Le musée, qui autrefois me faisait songer au bordel, écrit-il dans son Journal 96 , serait plutôt pour moi aujourd' hui - une église. Solution ancienne : se dépenser ; solution nouvelle : se recueillir. »

La critique des défauts d'observation, la proposition d'une démarche rationnelle d'analyse expriment pourtant une logique intellectuelle remarquable..., mais qui n'a influencé personne depuis Péron jusqu'à nos jours. Certes, on ne refait pas I'histoire a posteriori.

Il reste que si ces textes n'ont pas eu d'échos, et ont eu peu d'audience, c'est que, d'une certaine façon, ils étaient inutilisables. Comme va nous le montrer une [39] analyse plus poussée de leur contenu, une contradiction majeure non encore explicitée (et que seule une pratique de terrain conséquente pourra permettre d'expérimenter) les traverse: comment un sujet peut-il poser un autre sujet en objet de connaissance s'il lui est non seulement identique mais, comme on l'a vu, égal au moins en droit ? La méthode vise à instaurer une continuité et une rupture entre le sujet actif de la recherche et les objets de ses enquêtes. Or l'idéologie de la rupture du sens commun (l'antiethnocentrisme) s'oppose à celle de l'identification et de l'assimilation. La méthode de l'analogie du Même s'oppose à l'objectivation de I'Autre tout comme la théorie de l'entendement s'oppose à une grammaire historique ou à une sociolinguistique 97 . Dans la mesure où aucun

95 Cf. T. KROEBER, Ishi, Testament du dernier Indien sauvage de l'Amérique du Nord, Paris, Plon, 1971.

96 Cf. M. LEIRIS, Journal 1922-1989, Paris, Gallimard, 1992, (édition de Jean Jamin).

97 La relation entre la linguistique et l'anthropologie permet d'éclairer les conditions d'apparition de cette dernière. L'intérêt fondamental que les Idéologues portent à la langue se retrouvera chez E. B. Tylor par l'intermédiaire de la recherche d'une langue naturelle. Ce sont les travaux de l'abbé Sicard sur les sourds-muets qui serviront de base à ces nouvelles interrogations. Lire à ce su- 
des termes de cette contradiction n'a la force de prendre le pas sur un autre, nous sommes confrontés à une espèce de machine infernale sans détonateur. L'anthropologue et le sauvage ne sont peut-être que deux positions réciproques d'un même principe d'observation: «Il serait peut-être aussi intéressant pour l'histoire de l'homme de savoir ce que pensait le charpentier dans la circonstance où il se trouvait que de connaître le motif qui excita la curiosité des naturels », écrit Baudin à Jussieu (cf. infra).

\section{Un nouveau discours de la méthode 98}

\section{Retour à la table des matières}

Les textes que nous présentons ici parlent d'eux-mêmes, mais, audelà de la sémantique propre au langage des Idéologues, il y a des constantes et des contradictions qu'une lecture simultanée et comparée permet de mettre au jour. "L'air du temps » est néanmoins suffisamment bien marqué dans ses grandes lignes pour qu'on en reformule les propositions essentielles. Notre découpage entre idéologie, méthode et théorie relève plus d'une commodité de présentation. À ce titre, il est bien sûr arbitraire et les éléments dégagés peuvent s'envisager sous plusieurs angles: notre classification ne vise qu'à mettre en lumière l'aspect le plus significatif.

L'idéologie exprime le contexte social, voire politique, de la découverte: ce qui justifie le type d'approche mis en œuvre, le fondement social, voire politique, de toute relation d'observation. La gloire politique (et précoloniale avant la lettre de la science française!) explicite

jet H. BENSON, British Social Anthropologists and Language, Oxford, Clarendon Press, 1974, et notamment pp. 17-19. Cela dit, c'est chez les linguistes « génératistes » qu'une telle conjonction méthodologique va se réaliser: cf. $K$. HALE, « Some Questions about Anthropological Linguistics : the Role of Native Knowledge », in D. HYMES (ed.), Reinventing Anthropology, New York, Pantheon Book, 1973.

98 Nous donnons directement les citations sans les référer systématiquement: (Mem.) renvoie à l'introduction de Jauffret et (Mus.) à son projet de musée, textes reproduits infra. 
l'attention portée aux conditions du contact avec des [40] peuples étrangers. Mais la réflexion sur le contact tout comme le contenu méthodologique et théorique de l'observation de l'homme balance entre l'ethnocentrisme et l'anti-ethnocentrisme. C'est cette réflexion se contredisant elle-même qui signale l'ouverture méthodologique fondamentale de tous ces textes.

L'utilité morale (et donc politique) de la science ne fait aucun doute. Pour Jauffret (Mem.) I'homme doit s'estimer pour chercher à se connaître: «La Société [doit chercher] à relever la dignité humaine ». "L'avancement de la science » et «le bonheur des hommes» doivent marcher d'un même pas. Ce projet qui permet à Jauffret de critiquer "l'affreux régime qui pesa quelque temps sur la France » sert de fait à justifier une entreprise nationale 99 . Que ce soit Gérando ("De nos connaissances sur I'histoire physique et morale du monde multipliées et agrandies, du nom français porté sur des rives inconnues ») ou bien Péron (« Ne serait-il pas glorieux pour la nation française de devancer les autres peuples... ? »), pour tous les «observateurs de l'homme» l'amélioration des connaissances va de pair avec la diffusion des Lumières françaises. C'est cette volonté de puissance qui finit par fonder l'anthropologie car, comme l'explique Gérando, il faut promouvoir l'étude de soi-même malgré l'égoïsme de l'époque. Et pour se connaître, il faut établir des rapprochements, des comparaisons. En allant à l'autre bout de la terre, on remonte aussi dans le temps: on aurait tort du coup de mépriser nos ancêtres contemporains. Bien sûr il s'agit de nos ancêtres, mais Gérando comme Jauffret parlent de " ces peuples que méprise notre ignorante vanité », de « ces peuplades qui méritent si peu l'injurieux mépris que nous avons pour elles! ». Ces peuples, «qui n'ayant pas joué de premier rôle dans l'histoire, sont presque tout à fait inconnus » (Jauffret, Mem.), en nous instruisant nous permettent à leur tour de les instruire. Cette dialectique de la connaissance et de l'ignorance réciproques est tout à fait remarquable et permet de lier épistémologie du processus scientifique et acculturation. De fait, la logique d'une anthropologie appliquée est à l'œuvre:

99 Mais, lorsqu'en 1804, la Société demandera à devenir Société impériale des observateurs de l'homme, la carrière de Jauffret se terminera de fait, et il ira s'exiler dans une bibliothèque de province. 
«Et lors même que nous ne verrions pas dans les peuples sauvages un utile objet d'instruction pour nous-mêmes, ne serait-ce pas assez des nobles sentiments de la philanthropie, pour nous faire attacher une haute importance aux communications que nous pouvons former avec eux? Quel plus touchant dessein... que de leur tendre la main pour s'élever à un état plus heureux? 100 ». Et plus loin, il suggère « que si l'on trouvait quelques moyens de faire passer les peuples sauvages à l'état de pasteurs ou d'agriculteurs, on ouvrirait sans doute devant eux la route la plus sûre qui puisse les conduire aux avantages de la civilisation ». Nous voici donc, avant la lettre, en face d'une anthropologie économique appliquée au développement.

Ainsi, la situation de contact devient-elle le lieu d'une certaine réciprocité mais qui peut nous apparaître comme un échange inégal. Les conditions sociales et militaires [41] des rencontres sont évoquées inégalement. Pour une raison assez évidente liée à sa situation de praticien, c'est Baudin qui nous offre les réflexions les plus justes sur ce point. Il faut tout d'abord remarquer qu'il est difficile de produire une description simple et objective du contact : ainsi Péron voit-il les choses différemment de Baudin qui critique Labillardière. La bonté ou la méchanceté des sauvages sont autant affaire de perception idéologique des choses que de violence ou de retenue manifestes. Gérando nous laisse deviner l'importance de l'aspect armé des contacts puisque « on ne peut se plaindre que les voyageurs ordinaires [souligné par nous, J.C. et J.J.] nous laissent ignorer ni la nature des armes que les sauvages emploient ni l'usage qu'ils en font ». Et Gérando va plus loin puisqu'il consacre tout un paragraphe à la sociologie de l'accueil fait aux Européens ${ }^{101}$. Que la paix soit nécessaire à une bonne observation et que la violence ne soit pas la meilleure voie vers la «pacification» nous est confirmé par Baudin: "L'expérience qui d'ailleurs m'avait appris que la supériorité en force n'est pas toujours l'unique ressource à employer pour se garantir des pièges de l'homme de la nature... »

100 Il définit ensuite ce qu'il faut apporter et ce qu'il ne faut pas apporter aux sauvages.

101 Cf. le paragraphe intitulé : «Étrangers, hospitalité ». 
Nous voyons déjà que ces chercheurs favorisaient selon les problèmes à résoudre une position ethnocentrique ou anti-ethnocentrique. Le privilège de celui qui établit le contact est malgré tout manifeste. Mais cette ambivalence ou cette contradiction au cœur de la relation de contact est rarement théorisée en tant que telle. Nous ne pouvons que relever des signes de cette dualité. La volonté comparatiste commence par la comparaison la plus évidente et la plus motivée: celle avec nous-mêmes. Que ce soit Gérando (à propos des sensations il écrit au point 3: «Quel est le degré de développement de chacun de leur sens comparé à celui qu'on rencontre ordinairement parmi nous ? ») ou Péron qui évoque «le défaut de civilisation», le terme final des comparaisons ne fait pas de doute. De façon plus sophistiquée, Cuvier nous explique que pour décrire le corps humain, il faut faire abstraction de tous les artifices « culturels» de décoration 102. Ce choix de l'anatomie contre les techniques du corps apparaît comme celui de la nature contre la culture. Mais on sent bien que l'objectivité anatomique se double d'un ethnocentrisme corporel, car Cuvier ne reconnaît pas dans la nature les effets des autres cultures. Quant aux indices plus particuliers, ils détonnent parfois dans cette atmosphère de la distanciation et de l'objectivité. Citons l'évidence pour Péron et Baudin du fait du cannibalisme: I'attrait de Jauffret (Mem.) pour la physiognomonie : I'assimilation implicite des sourds-muets, de l'homme naturel (sans éducation) aux sauvages. Mais le lecteur aura senti que ces signes épars ne font pas encore une doctrine. L'antiethnocentrisme est tout aussi évanescent.

Le même Cuvier, qui récuse la culture des autres, note pourtant fort justement qu'il convient de se méfier des dessins faits par des Européens: «Les dessins qui se trouvent dans les voyages modernes quoique faits sur les lieux, se ressentent plus ou moins des règles et des proportions que le dessinateur avait apprises dans les écoles d'Europe, et il n'en est presque aucun sur lequel le naturaliste puisse assez compter [42] pour en faire la base de recherches ultérieures. » Malgré le sens de la comparaison, les remarques sur les peuples fausse-

102 «Les costumes, les marques par lesquelles la plupart des sauvages se défigurent, et que les voyageurs ordinaires ont tant de soin de nous transmettre ne servent qu'à masquer le véritable caractère de la physionomie. » 
ment méprisés témoignent d'une volonté de respecter la différence. L'attitude à l'égard des spécimens vivants à rapporter du voyage est encore une fois (et d'une certaine façon paradoxalement) à l'honneur de Cuvier qui s'y oppose (« il ne nous est pas permis, même lorsque nous le pourrions de sacrifier le bonheur, ni même de violer les volontés de nos semblables pour satisfaire une simple curiosité philosophique »), alors que Gérando 103 et Jauffret y sont passionnément favorables. Dans leur cas la volonté d'acculturation et d'assimilation, qui ne peut être que bénéfique (personne n'en doute un instant), est la meilleure justification d'une anthropologie appliquée. L'idéologie antiethnocentrique est donc aussi peu cohérente que l'idéologie ethnocentrique. Mais il n'en demeure pas moins que le vent souffle du côté du doute, de la distanciation et du respect des sauvages. Les défauts stigmatisés par Gérando montrent la profondeur et la précision de l'accusation du regard sans conscience de soi, du regard sans sympathie pour son objet.

La liste des défauts établie par Gérando est la première définition - en creux - des principes d'une nouvelle science de l'homme, empirique et non plus spéculative. C'est ce point que Jauffret met en lumière dès le début de son Introduction aux mémoires de la Société des observateurs de I'homme ${ }^{104}$. Gérando caractérise lui-même cette science comme " une science naturelle, une science d'observation». Le modèle de cette anthropologie comparée est la comparaison anatomique. Nous sommes encore loin de la réduction des faits sociaux à des choses mais en assignant, sans trop savoir comment aller plus loin, une positivité empirique à l'homme social et culturel, les « observateurs de l'homme » définissent un nouvel objet. Gérando va nous décrire son contenu assez longuement, mais il commence par un Discours de la méthode qui est plus une critique qu'une technique de l'observation.

103 Gérando est même partisan de ramener une famille entière, « image en petit de cette société », et surtout des jeunes.

104 «La Société, par son titre, annonce de quelle manière elle croit pouvoir arriver à une connaissance plus approfondie de l'homme. Son plan est surtout de recueillir beaucoup de faits, d'étendre et de multiplier les observations, et laissant de côté toutes ces vaines théories, toutes ces spéculations hasardées... » 
C'est cette discordance entre un objet défini de façon assez commune 105 et une logique du doute et de la précaution descriptive qui a probablement rendu ce texte inopérant. Comment reconstituer le soubassement épistémologique de ce passage qui ne constitue qu'à peine plus d'un dixième des Considérations ... ?

Le premier défaut est celui d'un empirisme mal compris: tous les faits ne sont pas rapportés. Qui plus est, ce ne sont pas les faits qui permettent une explication qu'ont relevée les voyageurs précédents. $A$ mal rapporter les formes et les effets on s'interdit de comprendre les causes. Si l'on se fie aux apparences, c'est qu' on a mal recueilli les faits : c'est qu'on a été mal informé. Le subjectivisme méthodologique [43] est un défaut particulièrement grave. Ce subjectivisme peut être considéré comme méthodologique parce que l'observation est menée sans ordre, sans hiérarchie des modalités de la collecte des faits. Il faut une espèce de théorie monographique pour être complet et donc objectif. Mais qui dit théorie dit explication, et c'est ici que le préjugé idéologique intervient; il faut expliquer d'après les faits et non d'après nos idées ou notre comportement: «Ils font raisonner le sauvage à notre manière lorsque le sauvage ne leur explique pas lui-même ses raisonnements ». Les observateurs précédents ont fait un mauvais usage de l'analogie. Ce défaut vient également de l'incertitude du langage employé : au lieu de descriptions factuelles précises, on a des impressions subjectives générales. Gérando montre ici toute la pertinence de la critique du sens commun des termes. Après le langage, ce sont les circonstances du contact qui induisent en erreur. Cette « sociologie du contact »(pourrait-on dire), donc les réactions des sauvages, donc les idées que l'on se fait de leur nature, suggère une indispensable maîtrise de soi, à la fois intellectuelle et émotionnelle, du futur « observateur de l'homme ». Elle laisse déjà entendre que ce qui compte pour une bonne observation, c'est la durée et la connaissance de la vie quotidienne 106 . Le contact est trop souvent l'exceptionnel qui déconcerte.

105 À l'exception bien entendu de la place accordée au langage. Voir infra nos remarques sur ce point.

106 «Pouvoir s'initier aux rapports ordinaires que ses membres ont entre eux. » 
Avec le septième défaut nous revenons au problème de la communication de l'information (sujet chéri des Idéologues, rappelons-le), c'est-à-dire aux rapports entre nature du contact et nature de la langue. On ne sait rien sur les circonstances linguistiques de la collecte des voyageurs. Des questions mal posées, des noms pris au hasard font que la bonne ethnographie suppose une bonne linguistique et surtout une bonne sociolinguistique 107. Car sans cela on ne peut saisir les traditions et l'âme des peuples étudiés. Le superficiel et l'anecdotique prennent le pas sur la recherche des «traits les plus secrets et les plus essentiels de leur caractère ». Ce défaut encore une fois relève plus d'un empirisme mal compris que d'une absence de théorie. La conclusion logique de l'ensemble de cette critique est évidemment cette fameuse anticipation de l'observation participante.

On voit combien la qualité de la description est la préoccupation centrale de Gérando comme elle a été celle de Volney. Cette description dépend de la nature du regard, du point de départ possible de la description. Ainsi, dans le texte sur le Chinois Tchong-A-Sam, Jauffret et Leblond posent-ils que la meilleure méthode consiste à inverser le regard: faut-il «enseigner à l'étranger le français» ou bien « apprendre de lui sa langue »? Cela dépend de son origine sociale : s'il est d'une « classe obscure » il n'a rien à nous rapporter, alors que doté d'une "éducation soignée », on retrouvera sa culture en apprenant sa langue. Passage fascinant sur lequel il y aurait beaucoup à dire, et où l'on retrouve une sociolinguistique qui précise son [44] point de vue de classe: seuls ceux qui sont bien éduqués savent quelque chose et peuvent le faire savoir, savent en tout cas l'exprimer, le formuler. La communication sociale est donc censée ne pouvoir s'établir qu'avec un informateur dont le niveau culturel est homologue à celui de l'observateur. Si l'on invite l'observateur à devenir le « concitoyen » des sauvages, il est parfaitement entendu que cette citoyenneté ne peut être que de haut rang! L'éducation, dont on sait qu'elle a été un souci majeur des Idéologues dans la mesure où elle leur permettait de penser l'ordre politique en même temps que l'ordre moral, est au savoir ce

107 Plutôt qu'une ethnolinguistique (aux sens français de ces disciplines). Nous pensons aux travaux de D. Hymes et à son ethnographie de la communication. 
que la langue est à la culture: leur condition. Ce qui signifie en clair que le savoir - ce faisant le capital linguistique qui à la fois le contient et le véhicule - est socialement distribué et hiérarchisé. Ce qui signifie également que la compétence cognitive et la performance linguistique, du fait des liens organiques qui leur sont prêtés, sont inséparables de rapports de force sociaux qui déterminent leur acquisition et leur expression. En somme, toute société aurait ses mandarins... 108 Et le Sauvage, le Persan ou le Chinois ne sont plus envisagés sous le seul angle d'une relation métonymique avec leur société d'origine. Sur ce point Gérando sera formel: «Les observateurs, écrit-il, ne doivent pas se borner à établir des recherches sur un seul individu [...], il est nécessaire de les répéter sur un grand nombre et de comparer les résultats qui en naîtront. Les voyageurs ne nous présentent ordinairement dans chaque pays qu'un type simple et commun, auquel ils supposent que se rapporte à la fois une peuplade entière. N'y a-t-il donc aucune variété entre les divers membres d'une société sauvage?»

Mais si Gérando consacre encore d'autres passages pour définir les précautions à prendre (cf. le paragraphe du même titre) lors des interrogations qu'il formule, c'est le praticien Baudin qui nous offre avec bon sens (mais aussi avec sa réserve) deux constatations critiques au sujet des « observateurs professionnels» de son expédition: il ironise sur les savants qui ne manqueront pas de transformer des dents cassées en dents limées et qui concluent que, parce que les naturels regardent souvent le soleil, celui-ci est leur divinité. On sent néanmoins combien ces précautions et cet esprit critique sont pleins de bonnes intentions et demeurent au seul plan des idées. Proposer une nouvelle démarche est une chose, la pratiquer en est une autre.

La notion de terrain est encore vague, mais elle existe indubitablement. Gérando nous a dit qu'il faut vivre parmi les sauvages. Péron luimême laisse entendre que l'expédition doit « séjourner longtemps » en divers endroits. Mais le terrain n'est pas du tout synonyme de promenade d'agrément. Gérando, en saluant les futurs voyageurs dans sa péroraison, évoque «I'image des fatigues, des privations, des dangers, qui vous attendent, et de ce long exil auquel vous vous êtes volontai- 
rement condamnés ». Mais c'est évidemment Baudin qui, ayant eu à gérer l'expédition et les humeurs des scientifiques, est le plus clair à ce sujet. Dans sa lettre du 4 floréal de l'an IX (cité dans la note 2 à son texte), il dit notamment à leur propos qu'ils sont [45] « peu accoutumés aux privations habituelles de l'homme de mer ». Et ses considérations sur les conditions du contact montrent que le «bon homme de terrain » (de contact) est celui qui sait maîtriser ses nerfs, ses émotions et son imagination.

À récapituler ces bribes de mise en condition ethnologique, aucune idéologie, aucune méthode fondatrice ne se dégage avec cohérence. Les six pages de Gérando ont indubitablement un caractère tout à fait exceptionnel. Qui plus est le lien à la théorie est aussi des plus ténus. Seule la recherche de la langue peut servir de fondement commun: elle seule définit la possibilité de l'observation participante tout comme la possibilité de l'histoire de la génération des idées humaines. Il $s$ 'agit de fonder un monde empirique à la place de fantasmes idéologiques et subjectifs. Il faut donc remplir un vide: l'exploration n'est pas seulement la possibilité d'un comparatisme puis d'une identification-assimilation, c'est aussi le plus sûr moyen de redessiner la condition humaine. Mais ce volontarisme empirique est aussi porteur de théorie. Après la méthode viennent les thèmes et domaines de l'observation. Dans certains cas, des définitions judicieuses confirment la richesse de l'esprit anthropologique du XVIIIe siècle finissant.

Les textes de la Société des observateurs de l'homme sont des textes «théoriques» au sens fort du terme même s'ils ne sont que partiellement destinés à élaborer des concepts. Bien sûr l'éloge de l'empirisme s'accompagne d'un refus des « vaines théories», mais Gérando est le premier à réclamer des faits véritablement explicatifs et le statut assigné au langage en fait un véritable opérateur théorique. Il n'y a pas de conception - du moins sémantique - uniforme de la science. Mais les définitions proposées tant de la science de l'homme que du scientifique chargé de l'exercer expriment déjà les ambiguiités et les contradictions que l'anthropologie va révéler au cours du siècle et demi suivant. Jauffret (Mem.) rappelle la définition la plus globale 
du projet des «observateurs de l'homme», c'est celle de «I'Idéologie », de «I'histoire philosophique de l'esprit humain».

C'est dans ce même état d'esprit que paléontologie, préhistoire, histoire ancienne et ethnologie semblent être les branches de "I'anthropologie comparée». Gérando partage cette définition «philosophique » et totalisante. Péron est le seul à considérer que l'objet nouveau de la recherche relève de la médecine ${ }^{109}$, ce que semble contester Jauffret (Mem.): "Par exemple, l'observation de I'homme physique embrasse l'anatomie et la physiologie, la médecine et l'hygiène: mais à cet égard la Société ne perdra jamais de vue que son but est de $n$ 'approfondir ces différentes sciences qu'en ce qui touche à l'histoire naturelle de l'homme proprement dite». Lorsque les « observateurs» désignent le chercheur, il leur arrive d'employer une expression bien plus significative de leur pensée et de leurs projets : ainsi [46] Gérando parle du «philosophe » mais surtout du « voyageur vraiment philosophe » 110 alors que Jauffret évoque le «naturaliste » et que Péron se définit comme « un médecin philosophe».

L'ordre des sciences va nous introduire à l'ordre des thèmes et des domaines. Pour Jauffret (Mem.), le physique, l'intellectuel et le moral sont inséparables. Sa hiérarchie des sciences se présente ainsi : anatomie, physiologie, médecine, hygiène, histoire, antiquités, mœurs et usages, morale et législation. L'ordre des domaines de ce nouvel objet d'études nous est détaillé par Gérando. Cet ordre est également un découpage conceptuel et c'est pourquoi nous pouvons légitimement parler de théorie: certains thèmes sont d'ailleurs mieux construits que d'autres. Le point de départ (qui est aussi le cadre explicatif) est la langue et la pensée qu'elle exprime: «Ce n'est pas seulement parce que cette étude est de toutes la plus importante en elle-même, c'est

109 «[...] quel théâtre plus propre à faire des observations neuves et intéressantes, que ces contrées immenses sur lesquelles les voyageurs et les historiens ont dit si peu de choses, et les médecins rien du tout encore... ? 》 Cette médecine étudie le corps puis les maladies dans leur contexte somatique social et culturel.

110 C'est ce que J.J. Rousseau proposait implicitement dans une des notes - (J) de la première partie de son Second discours où il affirme que des philosophes comme Diderot ou d'Alembert feraient de bien meilleurs observateurs que les voyageurs remplis de préjugés. 
encore parce qu'elle doit servir de préliminaire et d'introduction à toutes les autres.» Il précise même plus loin: «[...] ils tâcheront $d$ 'arracher en quelque sorte au sauvage le secret de son histoire intellectuelle et de nous transmettre le journal de la génération de ses idées. »

La table des matières que nous avons reconstituée est significative d'un ordre d'importance :

Table des matières synoptiques des Considérations... de Gérando *

INDIVIDU

$\begin{array}{cc} & 7 \mathrm{p} . \\ \text { Langue et signes } & 41 \mathrm{p} . \\ \text { État des sauvages } & 10 \mathrm{p} . \\ \text { Individu } & 31 \mathrm{p} . \\ \text { Société } & 13 \mathrm{p} . \\ & 15 \mathrm{p} .\end{array}$

SOCIÉTÉ

$\begin{array}{ll}\text { Maladies } & \\ \text { Éducation des enfants } & 2 \mathrm{p} . \\ \text { Idées } & 2 \mathrm{p} . \\ \text { Croyances } & 2 \mathrm{p} . \\ \text { Psychosociologie } & 3 \mathrm{p} .\end{array}$

Vie solitaire $0,2 \mathrm{p}$.

Société domestique

$2 \mathrm{p}$.

Société générale

$12 \mathrm{p}$.

politique

$4 \mathrm{p}$.

civil

$0,7 p$.

religieux

$3 p$.

économique

$4 p$.

* à partir de l'édition originale du Mémoire. 
[47]

Gérando consacre six fois plus de place à définir ce qu'il y a à observer qu'à la critique des défauts des observations passées. Mais sur cette quarantaine de pages, un quart détaille le rôle de la langue et construit sa signification théorique. Le reste (l'état du sauvage) est pratiquement consacré pour moitié à l'individu et pour moitié à la société. C'est dire finalement que seulement un quart de l'ensemble du texte de Gérando est consacré à des thèmes d'anthropologie sociale et culturelle proprement dite. Il distingue fort classiquement pour l'époque la vie solitaire, la société domestique et la société générale. L'ordre des rapports de cette dernière montre la double préoccupation du pouvoir (despotisme du politique, ténèbres des superstitions religieuses) et du commerce (l'économie). Cette détermination « en dernière instance " n'est même pas significative textuellement puisque dans le cours de la démonstration il intervertit le religieux et l'économique.

De son côté Jauffret propose à sa manière des ordres de réflexion thématique. Ainsi l'introduction aux mémoires de la Société des observateurs de l'homme annonce une topographie anthropologique (sorte de géographie humaine des genres de vie avant la lettre), un dictionnaire des signes, une expérience d'éducation de l'homme naturel, un dictionnaire comparatif des langues. Par ailleurs, dans son projet de Muséum, ses sept catégories sont autant de branches d'une anthropologie comparée. Il s'agit en effet de l'homme considéré. «1) en simple naturaliste ; 2) dans l'exercice de sa sensibilité extérieure ou de relation: 3) dans le double exercice de sa moralité et de son esprit: 4) dans l'exercice de la locomotion; 5) dans l'emploi de ses moyens de nutrition et relativement au fond de subsistance qu'il a trouvé, créé, conquis ou modifié : 6) dans ses rapports avec l'atmosphère: 7) dans l'exercice de la reproduction ». Ce dernier texte est très utile car, contrairement à la plupart des codes muséographiques qui portent sur la nature des objets, Jauffret part du cadre global de leur utilisation. Par ailleurs, certains des thèmes sont des branches anthropologiques bien conçues : ainsi la moralité (point 3) est-elle essentiellement le politique et le symbolique, fondements de toute anthropologie sociale. Quant au point 5, c'est une anthropologie économique complète et le 
dernier point est à la fois une génétique des populations et une démographie sociale. Il est évidemment facile de moderniser de telles classifications, mais nous voulons indiquer, ce faisant, la présence réfléchie d'une thématique construite malgré le refus d'une théorie qui doit céder le pas à l'empirisme des « vrais faits».

Gérando explique aussi le contenu de son découpage, mais il faut reconnaître qu'il procède plus par questions que par réflexion synthétique. Tout ce que nous pouvons relever c'est la place accordée aux « rapports politiques [...] qui servent de base à tous les autres». Enfin, il est significatif que la société civile (c'est-à-dire la propriété privée et le droit) n'occupe que les deux tiers d'une page. Gérando n'a pas pu penser la nature communautaire et individuelle (mais non privée) des modes d'appropriation des produits chez les chasseurs-cueilleurs (ou les pasteurs) non plus que le règlement non-institutionnel des conflits. En effet, il se trouve bien en peine [48] de savoir quoi demander puisque visiblement il n'a aucune idée de ce genre de réalité sociale.

Ce découpage thématique, ces définitions qui n'en sont pas exactement, ne correspondent pas à une interprétation historique des faits. Malgré la perception temporelle des rapports humains, la recherche ne débouche pas sur un historicisme (que le XIXe siècle va magnifier jusqu'à l'absurde). Gérando évoque les âges de la société humaine, mais il ne nous dit rien du passage d'un âge à L'autre ${ }^{111}$. La seule fois où il aborde ce problème c'est à propos de l'absence de passage à l'agriculture. Tout ce qu'il peut proposer c'est « de chercher à pénétrer le motif d'une si singulière répugnance ». Cette explication d'un manque par un refus nous rappelle des théories assez récentes sur le refus de l'État par les sauvages ${ }^{112}$.

111 Contrairement à l'utopie rousseauiste, Gérando exprime déjà le positivisme latent de l'empirisme qui constate seulement les faits et ne comprend rien à I'histoire de leurs transformations. Sous ce rapport-là il est aussi une sorte de «précurseur» de Malinowski. Sur tous ces problèmes épistémologiques de l'enquête de terrain, on lira avec profit l'ouvrage de J.-C. JARVIE, The Revolution in Anthropology, Chicago, Gateway, Edition, 1969.

112 Cf. les critiques des travaux et hypothèses de P. Clastres et J. Lizot dans J.-L. AMSELLE (éd.), Le Sauvage à la mode, Paris, Le Sycomore, 1979. 
Il n'est pas besoin de répéter combien la langue comme instrument et témoin de la pensée des sauvages est l'objectif stratégique, le médium parfait et l'idéal épistémologique des Idéologues. L'observation participante est pensée pour la première fois pour cette raison et l'intérêt porté à l'enquête linguistique et sociolinguistique subordonne aisément les balbutiements ethnologiques à une recherche des opérateurs logiques de la langue (langage d'action et langue articulée). À la fois structuraliste et "généraliste», la conception linguistique des observateurs de l'homme est la partie la plus élaborée de leur anthropologie, que ce soit au niveau idéologique, méthodologique ou théorique. Comme l'écrit Leblond dans son rapport sur le Chinois Tchong-A-Sam : «Combien il était puissant [l'intérêt] [...] de mettre en action une grammaire vivante de la langue près de laquelle échoue tout l'orgueil de nos savants! » Cette obsession linguistique permet d'expliquer une des constantes de l'anthropologie française orientée vers l'aspect social et culturel de la réalité. Comme l'écrit $H$. Henson dans sa conclusion: "La cause principale d'un développement différent en France provint peut-être de ce que l'anthropologie ne donna jamais naissance à une idéologie empirique, à la différence de la GrandeBretagne. Bien qu'il y eût un certain nombre de recherches de terrain, on était loin d'accorder autant d'insistance à la rigueur des méthodes de collecte des données comme en Grande-Bretagne, ou même en Amérique. Linguistique et anthropologie demeuraient dans la sphère de la philosophie ${ }^{113}$. »

[49]

Comment peut-on donc caractériser les ouvertures théoriques implicites aux études des observateurs de l'homme? L'anthropologie sociale (au sens classique du terme) a sans doute la portion congrue: elle s'identifie largement au politique, mais la conception appliquée des choses ne la transforme pas pour autant en une anthropologie politique. Il faudra une colonisation effective et surtout une idéologie nonassimilationniste pour qu' une telle pratique se développe. La tendance naturaliste et physique de l'anthropologie est encore une tendance totalisante qui correspond plus à l'anthropologie biologique telle qu'el-

113 H. HENSON, op. cit., 1974, p. 124. Il y aurait une étude assez approfondie à mener sur les rapports entre anthropologie et linguistique en France. 
le est conçue aujourd' hui. Mais Péron est une exception et, en restreignant le champ des études, il annonce l'anthropologie physique qui va dominer le XIXe siècle. Finalement, c'est au niveau du rapport entre langue et constitution de la pensée que l'apport théorique semble le plus considérable. La préoccupation pour l'éducation et les signes de I'humanité, le questionnaire linguistique 114 , I'attitude psycholinguistique sont autant de thèmes que le large courant de l'anthropologie symbolique a repris aujourd'hui. La triade Pouvoir-Corps-Signes résume bien l'horizon théorique des observateurs de l'homme. Le moins qu'on puisse dire c'est que leur programme est encore loin d'être achevé et que nous revivons, au moins métaphoriquement, à l'enseigne de la Société des observateurs de l'homme. Bien sûr, l'ensemble peut paraître désuet et dépassé et il serait abusif de nous réclamer de ces textes que ce soit pour la méthode ou pour les concepts qu'ils véhiculent.

Il reste que la force de leur imagination sociologique est à la hauteur de la censure qui les a frappés jusqu'à aujourd'hui, et les limites de l'anthropologie française paraissent de fait bien plus considérables une fois ces ancêtres - oubliés et surtout reniés -, remis à leur juste place et proportion 115 .

114 Voir par ailleurs les enquêtes linguistiques de la Révolution française et notamment celles de l'abbé Grégoire.

115 Il faut comprendre le terme limite dans les deux sens : ce que l'anthropologie n'a pas développé (sens négatif); ce à quoi elle s'est cantonnée (sens positif): d'une part son « retard » et son provincialisme, de l'autre son approche intellectualiste de la réalité sociale qui va de Gérando à Lévi-Strauss et aux marxistes. 


\section{Note sur la présente édition}

\section{Retour à la table des matières}

Plutôt que des «morceaux choisis», les textes réunis et présentés dans cet ouvrage constituent des « morceaux imposés», - imposés par la situation historiographique et par l'état des fonds archivistiques. En effet, depuis les publications d'Ernest-Théodore Hamy et de Georges Hervé, à la fin du XIXe siècle et au début du XXe siècle, lesquels ont été sans doute les derniers auteurs à avoir eu entre les mains les papiers de la Société des observateurs de l'homme, aucun de ces papiers n'a pu être retrouvé, soit que ceux-ci aient été détruits, soit qu'ils aient été conservés par les deux éditeurs puis dispersés ou perdus après leur mort du fait de leurs [50] ayants droit respectifs. Quoi qu'il en soit, nos démarches et nos recherches pour les retrouver ont été en vingt ans totalement infructueuses. L'édition que nous présentons ne peut donc être à proprement parler une édition critique, n'ayant pas eu la possibilité de remonter aux sources manuscrites ni, par conséquent, de vérifier pour certains des mémoires la fidélité des transcriptions effectuées par Hamy ou par Hervé. Chacun des écrits repris ici, qui se fondent donc sur les éditions d'Hamy et d'Hervé, est cependant précédé d'une courte introduction, composée en caractères italiques, rappelant les circonstances de sa rédaction, de sa découverte et de sa publication ou republication.

Lors de la première édition de ce livre (1978) - ceci dans une optique de commodité de lecture et, pensions-nous, dans un souci pédagogique -, nous avions cru bon de rassembler les mémoires de la Société des observateurs de l'homme en deux grandes parties que nous avions intitulées : «le Sauvage chez soi » et «le Sauvage ailleurs». À juste titre Benjamin Kilborne, dans un article puis dans une recension de notre livre 116 , avait critiqué cette organisation, considérant qu'elle introduisait une confusion dans la conception même que les «observa-

116 Cf. B. KILBORNE, « Le sauvage et I'homme», L'Ethnographie, fasc. $n^{\circ} 1,1980$, pp. 43-51; compte rendu de J. COPANS \& J. JAMIN, op. cit., 1978, Isis, 7, 4, 1980, p. 670. 
teurs de l'homme» se faisaient de l'homme sauvage, ou du moins risquait d'occulter cette sorte d'épistémologie génétique qu'ils avaient tenté de fonder en « inventant » une Science de l'homme; il eût mieux valu, observait-il, parler des «Observateurs chez eux» et des «Observateurs ailleurs ». Dans la présente édition, nous avons plutôt opté pour une organisation chronologique (à l'exception du texte de LouisFrançois Jauffret qui, comme son titre l'indique, introduit aux mémoires de la Société des observateurs de l'homme et se présente à la fois comme un programme d'étude et un « rapport d'activité » de la dite Société). L'édition originale de ce livre avait été introduite par une préface de Jean-Paul Faivre, l'un des premiers historiens de l'expédition du capitaine Baudin, qui rappelait en quelques pages les circonstances, les péripéties aussi bien que les défaillances et erreurs de celle-ci. Il s'attachait à réhabiliter la personnalité de son chef, lequel, méprisé pendant plus de cent cinquante ans, fut jugé responsable de l'extraordinaire coût en maladies et vies humaines que connut son équipage 117 et blâmé avec un certain acharnement par François Péron tout au long du récit que ce dernier fit du voyage aux terres australes. Le ton partisan, quelque peu hagiographique, de cette préface, notamment signalé par Paul Jorion 118 , n'est sans doute plus de circonstance aujourd'hui compte [51] tenu des progrès de l'historiographie sur cette expédition 119 et des travaux qui depuis ont été publiés à son sujet, faisant la part des choses, des hommes et des événements, donnant donc des principaux protagonistes une image plus nuancée, plus « contextualisée». Aussi, pour couper court à toute polémique, sommes-nous convenus de ne pas la reprendre.

117 CF. T. GUICHETEAU \& J.-P. KERNEIS, «Étude à travers les chrononavigrammes des responsabilités médicales de Baudin en Australie en 1801-1803 », in Les Français et l'Australie, Paris, Université Paris X-Nanterre, 1989, pp. 141156.

118 Cf. P. JORION, « Aux origines de l'anthropologie française », L'Homme, $X X$, 1980, pp. 91-98.

119 Cf. notamment F. HORNER, «The Baudin Expedition to Australia (18001804) $》$, in Les Français et I'Australie, Paris, Université Paris X-Nanterre, 1989, pp. 107-114 ; N. J. B. PLOMLEY, « The Baudin Expedition and the Tasmanian Aborigines in Tasmania», in Les Français et l'Australie, Paris, Université Paris X-Nanterre, 1989, pp. 133-140. 
Par rapport à l'édition de 1978, nous avons par contre ajouté deux écrits d'une part un texte de Gérando sur les rapports de J.M.G. Itard concernant "l'enfant sauvage de l'Aveyron» (rapports qu'il n'était pas utile de reproduire ici, ceux-ci ayant été publiés dans une édition de grande diffusion par Lucien Malson), d'autre part le mémoire de Péron sur les expériences dynamométriques et la force physique des sauvages de la terre de Diémen, extrait du Voyage de découvertes aux terres australes, mémoire dont la publication s'est imposée après la découverte par l'un d'entre nous, au Musée de l'Armée à Paris, de l'appareil de Régnier, semblable en tous points à celui que Péron avait transporté sur le terrain.

Notre introduction ainsi que les notes ont été entièrement revues et actualisées en fonction des études qui, depuis 1978, ont paru sur les Idéologues, plus particulièrement - mais aussi plus rarement - sur les observateurs de l'homme et sur les débuts difficiles mais passionnants de la discipline anthropologique dont témoignent tous les écrits réunis ici. 
[53]

Aux origines de l'anthropologie française. (1994) Édition revue et corrigée par les auteurs, 1993.

\title{
Texte 1
}

\section{Introduction aux Mémoires de la Société des observateurs de l'Homme (1800)}

\author{
Par Louis-François Jauffret
}

\section{Retour à la table des matières}

Cet écrit du secrétaire perpétuel de la Société des observateurs de l'homme a été lu dans la séance du 18 messidor de I'an IX. Un feuillet manuscrit biffé par l'auteur portait pour premier titre: Aperçu des travaux qui doivent occuper la Société des observateurs de I'homme (cf. R.-M. Reboul, Les Cartons d'un ancien bibliothécaire de Marseille, Draguignan, Latil, 1875, où cette introduction est publiée pour la première fois; elle a été reprise par Georges Hervé dans son article: «Le premier programme de l'ethnologie», Bulletin de la Société d'anthropologie de Paris, TX, 5ème année, 1909, pp. 479-487). Les notes en bas de page sont probablement de Jauffret lui-même, sauf mention contraire. Une grande partie des mémoires et des cours rédigés par Jauffret ont été retrouvés par R.-M. Reboul (par ailleurs auteur d'une biographie de Jauffret: L.-F. Jauffret (1770-1840), sa 
vie et ses œuvres, Paris, Six, 1869, et éditeur d'une Correspondance de L.-F. Jauffret, Draguignan, 1874). Ces mémoires et cours de Jauffret 120 sont conservés [54] à la bibliothèque de l'Académie de médecine à Paris, et sont classés en deux groupes .- a) mémoires pour servir à I'histoire naturelle, morale et politique du genre humain, b) cours d'histoire naturelle de I'homme, dont il ne reste que deux leçons, la

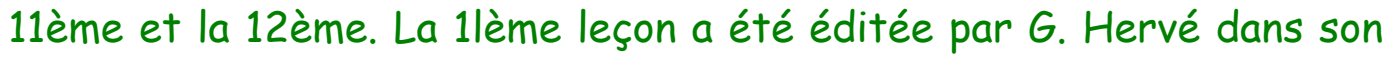
article: «Les premiers cours d'anthropologie», Revue d'anthropologie, vol. XXIX, 1914, pp. 255-276. Les thèmes abordés (mariage entre parents, origines de la polygamie, l'idée de nature chez les peuples sauvages, cannibalisme, suicides, idées des sauvages sur l'origine de I'homme, maquillage, mascarade, etc.) de même que leur qualité d'écriture et de réflexion font de ces mémoires et cours des documents précieux, dignes d'être exhumés, publiés et replacés dans I'histoire de l'anthropologie. Le nombre de feuillets manuscrits (plus de 350) ne nous permet pas, cependant, de les reproduire dans ce recueil.

Cette introduction aux Mémoires de la Société des observateurs de l'homme a été sans doute rédigée au début de l'an $X$, en tout cas après le départ de l'expédition du capitaine Baudin.

120 Selon Britta Rupp-Eisenreich, ces écrits attribués à Jauffret par I'historiographie du XIXe siècle seraient en fait des traductions anonymes d'une série d'essais de I'Allemand Cristoph Meiners développant son Précis de /'histoire du genre humain (Grundiss der Geschiste der Menscheit) publié en 1785 (cf. B. Rupp-EINSENREICH, « The "Société des observateurs de l'homme" and German ethno-anthropology at the end of the 18th Century ». History of Anthropology Newsletter, 10 (1), 1983, pp. 5-11; « Cristoph Meiners et Joseph-Marie de Gérando: un chapitre du comparatisme anthropologique », in D. DROIXHE et P.-P. Gossiaux (éds), L'Homme des Lumières et la découverte de l'autre, Bruxelles, éditions de l'Université de Bruxelles, 1986, pp. 21-47). La démonstration de Britta Rupp-Einsenreich est convaincante quant aux thèmes abordés et aux titres utilisés par Jauffret qui démarquent donc les essais de Meiners. Il reste cependant que, dans ces manuscrits, Jauffret fait souvent référence à lui-même et à quelques-uns de ses écrits. 
LA SOCIÉTÉ DES OBSERVATEURS DE L'HOMME dut s'occuper avant tout de bien mesurer la carrière qu'elle avait à suivre; de déterminer avec précision le genre de travaux qu'elle devait adopter. C'était le seul moyen de justifier son titre aux yeux du public et de montrer d'une manière sensible combien son existence peut être utile à l'avancement d'une science qu' on a toujours regardée comme la plus noble de toutes, quoiqu'elle ait toujours été la moins cultivée.

La Société, par son titre seul, annonce de quelle manière elle croit pouvoir arriver à une connaissance plus approfondie de l'homme. Son plan est surtout de recueillir beaucoup de faits, d'étendre et de multiplier les observations, et laissant de côté toutes ces vaines théories, toutes ces spéculations hasardées, qui ne serviraient qu'à envelopper de nouvelles ténèbres une étude déjà si obscure par elle-même.

Elle se propose d'observer I'homme sous ses différents rapports physiques, intellectuels et moraux, en ayant soin toutefois de se renfermer dans de certaines bornes.

Par exemple, l'observation de l'homme physique embrasse l'anatomie et la physiologie, la médecine et l'hygiène: mais, à cet égard, la Société ne perdra jamais de vue que son but est de n'approfondir ces différentes sciences qu'en ce qui touche à l'histoire naturelle de I'homme proprement dite. Cette direction particulière lui offrira les recherches les plus neuves, les plus importantes, et aura l'avantage de ne pas confondre ses travaux avec ceux des Sociétés spéciales de médecine et de chirurgie.

Ainsi, tandis que ces dernières Sociétés s'occuperont de leurs recherches ordinaires, et s'efforceront de perfectionner l'art de guérir, celle des Observateurs de I'homme jettera un regard attentif sur la physionomie des divers habitants de la Terre; elle étudiera les causes qui distinguent un peuple d'avec un autre, et qui altèrent [55] en divers pays la forme et la couleur primitives de l'espèce humaine. Il est de fait, comme le dit Camper, que, même au premier coup d'œil, non seulement le Nègre diffère du Blanc, mais le juif du chrétien, 
I'Espagnol du Français, le Français de I'Allemand, I'Allemand de I'Anglais ; bien plus, en se bornant à une nation particulière, et en la partageant en diverses régions, on reconnaît encore parmi les habitants de ces régions des différences marquées. Souvent les habitants d'une ville, ou même d'un village, ont une coupe de tête, une physionomie héréditaire qui les séparent de tous leurs voisins. Ces différences n'ont pas encore été assez observées : il appartient à la Société de les constater, et de recueillir, par le moyen de ses correspondants, un assez grand nombre d'objets de comparaison, pour que les faits qu'elle publiera là-dessus n'aient rien de vague ni d'incertain.

Ce ne sera que par la réunion successive de ces nombreux objets de comparaison, et par un travail complet sur l'anatomie comparée des peuples, que l'on pourra un jour caractériser d'une manière exacte les variétés de l'espèce humaine. Blumenbach pour justifier la division qu'il a faite du genre humain en cinq races principales, a publié la description et la figure de plusieurs crânes de divers peuples ${ }^{121}$, qui font partie de son riche cabinet anatomique. On doit lui savoir gré sans doute du soin qu'il a pris de rassembler ces premiers matériaux d'une histoire des variétés naturelles de l'homme, fondée sur l'observation, mais on doit attendre, et du temps et du zèle des voyageurs, une plus riche collection d'objets à comparer, avant de pouvoir hasarder une classification méthodique des différentes races. L'examen approfondi de la partie osseuse de la tête humaine est sans doute fait pour donner lieu à des rapprochements intéressants mais ces rapprochements seront équivoques tant que l'examen ne portera que sur le crâne d'un seul individu, pris au hasard sur la totalité d'un peuple, et que les observations n'embrasseront pas la totalité des régions.

Il appartient à la Société des observateurs de l'homme de remarquer les grands caractères qui distinguent cet être des animaux, et qui le placent bien évidemment à la tête de tout le règne organique. Par exemple, sa station sur deux pieds à corps vertical, que Rousseau a

121 Decades VI craniorum diversarum gentium, 1790-1828. [J.-F. Blumenbach (1752-1840), fut un des pionniers de la crâniométrie et l'un des premiers à classer l'espèce humaine en cinq races distinctes (NDE)]. 
voulu lui disputer, est trop bien démontrée par la description anatomique des parties qui la favorisent et la déterminent, pour que cette description soit étrangère aux travaux d'une réunion qui a en vue de donner à l'homme tout le développement moral dont il est susceptible, en lui restituant toute sa dignité physique.

Il est impossible d'étudier le corps humain sans être étonné de cette extrême flexibilité qui le rend propre à supporter également les climats les plus opposés, tandis que des animaux remarquables par une taille gigantesque et par une force extraordinaire ne peuvent outrepasser les bornes étroites du domicile que leur assigna la nature.

[56]

C'est la convenance du climat et la qualité de la nourriture qui tiennent chaque animal attaché à son domicile primitif. Celui dont l'estomac supporte une plus grande variété d'aliments, ou qui se contente d'une nourriture simple et grossière, supporte par cela même une plus grande variété de climats : mais qu'il est heureux pour l'homme que les animaux qui lui devaient être les plus utiles soient aussi ceux que la nature organisa de manière à occuper un plus grand nombre de latitudes! La faible brebis habite au-delà du cercle polaire, en même temps que sous la ligne de l'équateur, tandis que le rhinocéros et le tigre occupent à peine quelques degrés, que jamais ils n'ont pu franchir; le lièvre et le lapin affrontent les climats glacés du nord, tandis que le monstrueux hippopotame périrait s'il sortait du cœur de l'Afrique: enfin le bon et fidèle compagnon de l'homme, le chien, habite avec lui sous toutes les zones, et le Patagon, comme l'Européen, lui confie la garde de ses troupeaux : tandis que cet autre animal, que quelques naturalistes n'ont pas craint d'élever jusqu'à nous, parce qu'il a à l'extérieur une imparfaite ressemblance avec l'homme, est borné à quelques régions, et n'a pas même un domicile aussi étendu que la zibeline.

L'histoire naturelle de l'homme et des animaux, considérée sous ce point de vue géographique, fixera les regards de la Société. On ne peut l'approfondir sans reconnaître dans l'homme une grande supériorité d'organisation, et une sorte d'empire: sans être frappé du pouvoir qu'il a de forcer quelquefois la nature, en entraînant surtout à sa 
suite les espèces parasites, et en parvenant à acclimater certaines espèces indépendantes à une grande distance de leurs régions natales.

S'il existe des différences remarquables de peuple à peuple, même de famille à famille, il en existe de moins sensibles sans doute, mais cependant d'aussi réelles, d'individu à individu; et c'est l'étude approfondie de ces différences qui constitue la physiognomonie.

La Société a ici deux écueils à éviter: ou ce pyrrhonisme absolu, qui ne voit sur les diverses physionomies que des caractères insignifiants: ou cette confiance excessive, qui prétend en expliquer le sens et y lire aussitôt toute la destinée d'un homme. Il est sans doute avantageux pour la tranquillité publique et pour le bonheur des particuliers, que la physiognomonie ne soit encore qu'une science conjecturale: que le visage humain soit une espèce de masque aux yeux de ceux qui le regardent; et peut-être entre-t-il dans les sages vues de la $\mathrm{Na}$ ture que l'alphabet de ces caractères mystérieux qu'elle imprima sur le front de chacun de nous ne soit jamais révélé en entier: mais, tout en blâmant l'imprudent désir de vouloir déchiffrer chacun de ces traits, et la témérité de prétendre généraliser les inductions, la Société ne repoussera pas les observations sur un sujet si neuf et intéressant; elle se fera même un devoir de les publier, lorsqu'elles auront été dictées par un zèle prudent et éclairé.

On voit déjà que l'observation de l'homme physique est intimement liée à celle [57] de l'homme moral, et qu'il est presque impossible d'étudier le corps ou l'esprit d'une manière isolée.

Aussi les médecins que la Société compte parmi ses membres 122 ouvriront pour elle une source de recherches intéressantes, en épiant sans cesse avec des yeux observateurs l'influence des affections de I'homme sur le jeu de ses organes, et celle du jeu de ses organes sur ses affections: en développant l'action continuelle de l'esprit sur le corps, et celle du corps sur l'esprit; en remarquant enfin que les pas-

122 Jean-Noël Hallé, Philippe Pinel, Bouvier, Moreau de la Sarthe, Pierre Sue, Cabanis, Auguste Thouret, Pierre Lassus, Itard, Nysten, etc. 
sions de l'homme sont ses plus cruels ennemis, puisqu'elles seules fécondent le germe de presque toutes ses maladies.

Et qu'on ne pense pas que la morale seule puisse profiter de ces sortes de considérations! L'hygiène et l'art de guérir n'en tireront pas de moins grands avantages. L'hygiène, qui n'est au fond que la morale mise en pratique, écartera presque tous les maux dont l'homme est menacé, en lui démontrant que chaque maladie est presque toujours le produit d'un vice: l'art de guérir détruira, ou du moins neutralisera le plus grand nombre de ces maux, en combattant dans I'homme le mal moral, plus encore que le mal physique.

Après avoir esquissé d'une manière bien imparfaite les travaux que la Société a cru devoir indiquer au zèle de ceux de ses membres qui cultivent spécialement l'anatomie et la physiologie, la médecine et l'hygiène, donnons également une faible idée des travaux que se proposent d'embrasser ceux d'entre eux qui ont fait une étude particulière de l'histoire et des antiquités, et ceux qui, par des voyages lointains, ont acquis ou doivent acquérir un jour des connaissances étendues sur les moeurs et les usages des divers peuples.

S'il est un spectacle digne de fixer les regards des hommes, d'exciter la curiosité des uns, d'éveiller l'attention des autres, de commander l'admiration de tous c'est sans doute celui du genre humain, s'élevant dès les siècles les plus reculés à une supériorité d'industrie qui place bien évidemment notre espèce au-dessus de toutes les autres, ou plutôt qui en fait une espèce à part, dont la terre entière est le domaine et à laquelle toutes les autres sont subordonnées.

La Société, en jetant les yeux sur les diverses parties de l'ancien monde, remarquera la succession rapide et continuelle des générations qui l'ont habité tour à tour, ainsi que la hardiesse de l'homme, qui le couvrit de monuments, dont quelques-uns subsistent encore après une si longue suite de siècles; elle remontera jusqu'à ces époques où la tradition a placé le berceau des arts, des lois et des sciences, cherchera les traces de la grandeur humaine jusque dans les ruines qui attestent son néant, s'efforcera de démêler l'origine et les différentes 
migrations des peuples: et, tandis [58] que ses membres voyageurs 123 lui feront connaître les différentes nations qui occupent aujourd'hui la surface de la terre, ses membres historiens 124 lui feront connaître celles qui y brillèrent autrefois.

Des recherches suivies, des détails étendus sur les anciens peuples et en particulier sur ceux qui, n'ayant pas joué de premier rôle dans I'histoire, sont presque tout à fait inconnus, jetteront un grand jour sur I'anthropologie comparée; et, sous ce rapport, la Société a dû les recommander au zèle de ceux de ses membres qui cultivent la science des antiquités.

En même temps que les recherches sur les mours et usages des anciens peuples favoriseront celles que l'on pourra faire sur les mours et usages des peuples modernes, les observations des navigateurs sur les habitants actuels des diverses régions pourront fournir des lumières précieuses sur les premières époques de l'histoire du genre humain. Quoi de plus propre, en effet, à éclaircir les points les plus obscurs de notre histoire primitive, que de comparer ensemble et les mœurs, et les habitudes, et le langage, et l'industrie des divers peuples, de ceux surtout qui ne sont pas encore civilisés! Et quoi de plus satisfaisant, pouvons-nous ajouter, que de se livrer à une occupation semblable, que de lier, pour ainsi dire, connaissance avec un nombre infini de peuplades, qui méritent si peu l'injurieux mépris que nous avons pour elles!

Il fut un temps où le désir d'observer l'homme n'entrait pour rien dans l'exécution des voyages qu'ordonnaient les gouvernements. Rapporter des pays lointains des animaux, des végétaux et des substances minérales, voilà quel était l'unique motif de toutes les expéditions scientifiques. Quant aux expéditions commerciales, elles n'avaient pour but que d'aller au loin propager nos vices et déshonorer l'humani-

123 Bougainville, Patrin, Nicolas Baudin, Hamelin, Bernier, André, Michaux, Dolomieu, Levaillant, Sonnini, Maugé, Riedlé, Bissy, etc.

124 Walckenaer, Millin, d'Ansse de Villoison, Volney, Larcher, Papon, Pfeffel, Silvestre de Sacy, Pastoret, Mathieu-Antoine Bouchaud, Sacray, Marcel, SainteCroix, Clermont-Lodève, etc. 
té. La fin du XVIIIe siècle a ouvert une route nouvelle, et le commencement d'un siècle nouveau favorisera l'impulsion donnée. Marchant sur les traces de Cook, et de ce voyageur non moins illustre que la Société compte parmi ses membres 125 , des observateurs pleins de zèle, correspondants de la Société, sont allés étudier l'homme sur le vaste théâtre de l'univers. Éclairés sur les fautes principales commises par les anciens voyageurs, et sur les vides qu'ils ont laissés dans leurs relations, ils s'efforceront de faire ce que leurs prédécesseurs n'ont pas fait.

Les Considérations sur les diverses méthodes à suivre dans l'observation des peuples sauvages 126 , adressées par la Société au capitaine Baudin et aux observateurs qui l'accompagnent, contiennent un cadre dans lequel peuvent se rapporter toutes [59] leurs remarques. Ces considérations leur retraceront continuellement les principaux objets sur lesquels doivent se diriger leur attention et leur zèle, notamment les observations à faire sur les signes des sauvages, tant sur leurs gestes, avec lesquels les gestes des sourds-muets ont un si grand rapport, que sur leur langue articulée, dont les anciens voyageurs ont trop négligé l'étude, et qu'on ne peut espérer d'apprendre qu'en suivant l'ordre le plus conforme à la génération des idées.

Il appartenait à la Société de jeter les premières bases d'un ouvrage, dont l'importance ne peut être méconnue et dont le succès ira toujours croissant ; d'un ouvrage fait pour honorer tout à la fois, et la réunion qui l'aura entrepris, et le Gouvernement qui l'aura favorisé. On sait combien d'illustration valut à la Société royale de médecine le projet d'une topographie médicale de la France, qu'elle avait commencé d'exécuter. La Société des observateurs de l'homme a conçu le projet d'un travail analogue et non moins utile, d'une Topographie anthropologique de la France, et par suite, à l'aide de ses correspondants-voyageurs, d'une Anthro-pographie des différentes régions 127.

125 Il s'agit probablement de Bougainville (NDE).

126 Rédigées par J.-M. DE GÉRANDO, Paris, an VIII, in-4, $57 \mathrm{p}$.

127 Ce fut A.-L. de Jussieu, président de la Société en I'an IX, qui eut la première idée de cette Topographie anthropologique. 
Les observations topographiques sont d'une utilité indispensable pour déterminer d'une manière exacte l'influence du climat sur I'homme; et l'on n'ignore pas que cette différence qu'on remarque dans le physique et dans le moral des individus, tient fort souvent à cette influence; que l'état du corps éprouve des variations très grandes d'après les différentes saisons de l'année: que cette différence des saisons étant causée en grande partie par la manière dont les vents soufflent en divers temps, il en résulte que les vents ont aussi une influence marquée sur l'état du corps humain; que les habitants d'une ville située dans une plaine ou une vallée sont sujets à d'autres maladies que ceux d'une ville située sur une colline ou une montagne: que des aliments divers affectent diversement l'économie animale; et qu'ainsi des peuples pêcheurs doivent offrir des particularités qui les distinguent de ceux qui ne vivent que de leur chasse.

Outre les différentes observations que la Société attend du zèle des voyageurs qui correspondent dès ce moment, et de ceux qui correspondront par la suite avec elle, elle leur demande encore de seconder le projet qu'elle a conçu de réunir peu à peu, dans un Muséum spécial 128 , divers objets relatifs aux travaux dont elle s'occupe, et notamment tous les produits de l'industrie des sauvages, tous les objets de comparaison qui peuvent servir à faire connaître les variétés de l'espèce humaine, ainsi que les mœurs et usages des peuples anciens et modernes.

On ne peut se le dissimuler, un établissement de ce genre, si le gouvernement daigne seconder sa formation, doit devenir un jour aussi précieux à la science que piquant pour la simple curiosité. Et pourquoi n'obtiendrait-il pas toute la faveur [60] qu'il mérite? La Société le forme à peine, et déjà des particuliers généreux lui ont fait des dons. Des voyageurs, animés d'une noble ardeur pour les progrès de la Science de l'homme, des marins distingués et bienveillants, lui ont promis un tribut abondant d'objets intéressants et nouveaux. Des instructions particulières ont été données à ces dignes correspondants pour les diriger dans le choix de ces objets. La Société n'aurait donc

128 Ce projet fut réalisé en partie. Jauffret eut l'honneur de l'avoir formé. Il publia à ce sujet un Mémoire sur l'établissement d'un Muséum anthropologique, Paris, Gillé, s. d. [1803] (NOTE DE G. HERVÉ). Cf. infra. 
plus besoin que de quelques secours du Gouvernement pour être assurée de voir son Muséum, enrichi successivement des tributs d'un zèle sincère et d'une bienveillance active, tenir bientôt une place honorable parmi les établissements consacrés à l'instruction publique.

On a vu par tout ce qui précède que ce n'est qu'en recueillant une grande suite de faits, qu'en s'environnant d'une multitude d'objets de comparaison, que la Société veut procéder à la connaissance de l'homme. Elle n'abandonnera pas, cette route si sûre de l'observation, même en se livrant à l'étude des facultés de l'âme, à cette étude qui ne fut si stérile et si déprimée, pendant tant de siècles, que parce qu'on négligea jusqu'à nos jours de l'appuyer sur les mêmes bases que les autres sciences naturelles, c'est-à-dire sur l'observation et sur l'expérience.

Déjà dans la question que la Société a cru devoir proposer pour sujet du prix qu'elle adjugera dans le courant de l'an XI, elle a montré combien elle attache d'importance à obtenir une série d'observations bien faites sur les premiers développements des facultés de I'homme au berceau. Ce travail, aussi neuf qu'intéressant, indiqué par la Société aux vrais amis de la philosophie, est sans doute entouré de nombreuses difficultés. Mais ces difficultés ne sont pas insurmontables; et pourquoi, d'ailleurs, ne trouverait-on pas un certain attrait dans le plaisir et l'honneur de les vaincre? Pourquoi ne trouverait-on pas le même charme à considérer d'un ceil attentif la première lueur de l'esprit qui se développe, à tenir un journal détaillé des progrès de l'intelligence dans un enfant, à voir naître ses facultés l'une et l'autre qu'à épier les mœurs et l'industrie d'un insecte, qu'à observer la floraison de quelque plante étrangère? Ne serait-ce donc que lorsqu'il s'agirait de s'épier et de se connaître lui-même que l'homme cesserait tout à coup d'éprouver cette curiosité impatiente qui l'aiguillonne dès que ses yeux s'ouvrent à la lumière ?...

La Société possède dans son sein des hommes 129 qui, ayant approfondi avec succès l'idéologie, pourront diriger avec fruit tous les tra- 
vaux qu'elle jugera nécessaire d'entreprendre pour éclaircir cette science tout à la fois si noble et si dédaignée.

Que de secours n'obtiendra-t-elle pas de celui de ses membres 130 à qui l'art d'instruire les sourds-muets a de si grandes obligations! L'observation suivie de ces êtres, disgraciés de la Nature, des procédés inventés pour arriver jusqu'à leur intelligence, des signes par lesquels ils suppléent à la parole, de la pénétration avec laquelle ils saisissent les idées qu'on leur présente, l'étude philosophique de ce qui [61] se passait en eux avant l'époque de leur instruction, l'examen comparatif du développement des facultés dans plusieurs de ces individus, offriront à la Société une abondante moisson de faits aussi curieux que propres à faire connaître l'origine de nos idées, et à nous indiquer la véritable manière dont elles se ramifient.

S'il appartenait à la Société des observateurs de l'homme de s'entendre avec l'instituteur des sourds-muets, pour faire à plusieurs de ses élèves une suite de questions à leur portée sur l'époque qui a précédé leur instruction, leurs réponses, conservées avec soin et sans altération, deviendront des matériaux précieux pour une histoire philosophique de l'esprit humain.

Un travail intéressant que l'instituteur des sourds-muets a annoncé devoir entreprendre, et dont la Société hâtera, s'il est possible, l'exécution, c'est un Dictionnaire des signes ${ }^{131}$. Un pareil ouvrage sera un monument national. Comme il donnerait la traduction fidèle des signes qu'emploient les sourds-muets non encore instruits, pour se faire comprendre, la lecture en serait non seulement utile pour correspondre avec ces infortunés, et avec les peuples sauvages, mais le philosophe y suivrait avec une admirable facilité toute l'histoire de la génération de nos idées.

Un jour, la Société aura peut-être à examiner si, pour suivre d'une manière aussi neuve qu'étendue le développement progressif des facultés physiques, intellectuelles et morales de l'homme, il ne serait pas

L'abbé Sicard.

131 L'abbé R. SICARD (1742-1822) publiera en 1814 La Théorie des signes ou Introduction à l'étude des langues, où le sens des mots au lieu d'être défini est mis en action... Paris, Michaud, 2 vol. (NDE). 
convenable de tenter, avec l'autorisation du gouvernement, une expérience sur I'homme naturel, qui consisterait à faire observer avec soin, pendant douze ou quinze années, quatre ou six enfants, moitié de chaque sexe, placés dès leur naissance dans un même enclos, loin de toute institution sociale, et abandonnés pour le développement des idées et du langage au seul instinct de la nature 132 .

Il n'est pas douteux qu'un moyen sûr d'obtenir une suite d'observations, capables de concourir puissamment à nous éclairer sur le développement de nos facultés, serait de placer ainsi dès leur naissance, sous les regards de la philosophie, des enfants qui, isolés de nos mœurs, de nos institutions, de nos préjugés et même de notre langage, ne pussent agir et s'exprimer que d'après l'instinct et l'état que la nature donne à tous les hommes.

Psammétique, roi d'Égypte, voulut autrefois, au rapport d'Hérodote, faire tenter une éducation de ce genre. Akbai, empereur du Mogol, essaya aussi, il y a quelques siècles, de faire élever des enfants loin de toute société. Depuis ces essais informes, dont l'histoire n'assure même pas l'authenticité, aucune tentative analogue n'a été faite par aucun gouvernement. Une expérience sur l'homme naturel faite dans un siècle aussi éclairé que le nôtre, aurait donc sous tous les rapports le mérite de la [62] nouveauté. Les nombreuses difficultés attachées à son exécution ont pu seules jusqu'à présent effrayer ceux-là mêmes qui en ont le mieux apprécié les avantages. En effet, une pareille entreprise commanderait le sacrifice d'une vie entière. Il faudrait, en s'y dévouant, être assez jeune pour espérer raisonnablement de la conduire à son terme, assez philosophe pour se passer, pendant toute sa durée, de ce qu'on nomme les agréments de la société, assez désintéressé pour immoler sa fortune à sa gloire, assez à son aise pour n'être à charge à personne, assez en garde contre les systèmes pour observer sans prévention, enfin assez ami de la vérité pour tout dire et ne rien omettre.

132 « Une personne qui a connu M. Jauffret nous a assuré que cette expérience avait été tentée, et que les résultats en avaient été recueillis par l'un des membres de la Société, qu'elle n'a pu nommer. » (NOTE DE R. REBOUL). 
Mais de quel courage ne serait pas capable celui qui pourrait ouvrir son cœur à la noble ambition de tenter pour la première fois cette grande expérience, et essaierait par là de soulever un coin de ce voile mystérieux qui nous cache les secrets de la Nature! Des hommes, avons-nous déjà dit, sont pour le moins aussi intéressants à observer que des plantes ou des insectes. Pourquoi donc n'aurait-on pas, en se livrant à une étude aussi neuve, aussi intéressante que celle de l'homme lui-même, et le courage d'un Commerson, et la patience d'un Réaumur? 133

L'utilité réelle d'une expérience sur /'homme naturel, les problèmes difficiles qu'elle pourrait aider à résoudre, tant sur l'origine du langage que sur l'origine des idées mêmes et sur les notions fondamentales de l'esprit humain, la rendraient sans doute digne de la protection d'un gouvernement éclairé. Déjà bien des philosophes ont pressenti ses avantages, et ont désiré qu'un observateur courageux fût autorisé à la tenter. "Après tant de siècles écoulés, - dit Maupertuis dans sa Lettre sur le progrès des sciences, - pendant lesquels, malgré les efforts des plus grands hommes, nos connaissances métaphysiques n'ont pas fait le moindre progrès, il est à croire que, s'il est dans la nature qu'elles en puissent faire quelqu'un, ce ne peut être que par des moyens nouveaux et aussi extraordinaires que celui-ci. »134

Quels que fussent les résultats d'une expérience sur l'homme naturel, ils ne pourraient être indifférents. La nullité même des résultats serait utile à obtenir, parce qu'en éclairant sur l'infructuosité d'une éducation toute naturelle, elle nous apprendrait à chérir davantage le bienfait des institutions sociales auxquelles l'homme serait redevable de ce qu'il est aujourd'hui.

133 Ph. Commerson (1727-1773), naturaliste français, participa au voyage de Bougainville de 1766 à 1769, et rapporta de nombreuses collections et dessins. R.A. Réaumur (1683-1757), physicien et naturaliste français, fondateur de l'entomologie, connu entre autres pour ses travaux sur la digestion (NDE).

134 P.-L. MAUPERTUIS (1698-1759), Lettre sur le progrès des sciences, Paris, 1752 (NDE). 
Ce projet d'expérience nous conduit naturellement à parler des diverses observations à faire sur les langues, objet important que la Société a embrassé avec beaucoup d'ardeur, et qui peut lui fournir les résultats les plus précieux. La parole est, après la raison, la plus belle prérogative de I'homme. Si la nature lui eût refusé ce grand moyen de communication, il n'en fût pas moins venu à bout de peindre ses [63] idées. La langue des gestes, aussi perfectionnée qu'elle peut l'être, aurait suppléé, du moins en partie, à la langue parlée: mais combien la parole lui est plus avantageuse, et que ses effets sont plus prompts! $C$ 'est surtout elle qui distingue l'homme de cette foule d'animaux dont il est environné. Par elle, il communique avec une incroyable rapidité ses besoins, ses craintes, ses désirs, ses lumières: par elle, il sort de la sphère circonscrite où la nature l'avait placé, se met en rapport avec tous ses semblables, échappe avec eux à la barbarie, et parvient peu à peu au plus haut degré de civilisation.

Il entre dans les attributions de la Société de considérer comment les langues ont pu se former; de retrouver en quelque sorte le chemin que durent suivre les premiers hommes, pour combiner les éléments de la parole et les assujettir à des règles fixes. Ce serait une erreur de croire que les mots ont été faits au hasard. Une langue dont le hasard seul aurait combiné les principes ne serait qu' un vaste chaos.

C'est pour retrouver cette route que suivirent les premiers inventeurs des langues, que des observations exactes et multipliées sur les premières articulations des enfants seront très utiles à la Société. La nature nous porte dès l'enfance à exprimer, par des sons imitateurs, les bruits qui nous frappent et les cris des animaux qui nous environnent. Croasser, miauler, piauler, roucouler, aboyer, tinter, siffler, sont des mots qui n'ont pas été faits au hasard. On peut donc croire que tous les autres ont eu aussi primitivement leur raison, et que cette raison serait très sensible pour nous, si nous pouvions remonter à leur origine.

Il appartient à la Société, à ceux de ses membres surtout qui se livrent à l'étude approfondie des langues anciennes et modernes, de développer les grandes vues que renferme le célèbre traité du Président De Brosses sur la formation mécanique des langues. En lisant cet ouvrage lumineux, on croit être transporté au moment où les langues 
se formèrent. On voit l'instrument vocal produire des intonations imitatives, et les intonations radicales donner ensuite naissance à une multitude infinie de mots articulés d'une manière plus ou moins forte, plus ou moins rude, plus ou moins gutturale, plus ou moins douce, suivant le caractère des peuples 135 .

Tel était cependant encore le discrédit où était plongé l'art étymologique à l'époque où ce livre parut, que le président De Brosses n'en tira pas de son vivant toute la gloire qu'il avait droit d'en attendre. Sa réputation s'accroît surtout depuis quelques années, parce que les bons esprits semblent diriger aujourd'hui leurs efforts vers l'étude du langage, et que l'on commence à sentir que la connaissance des mots est comme la clef de la connaissance des choses.

La Société, en continuant les recherches de cet auteur sur le mécanisme des langues, en rectifiant quelquefois ses observations, en appuyant sur des faits nouveaux [64] et plus étendus les principes qu'il a établis, en obtenant par de nombreuses correspondances des notions bien ordonnées sur les divers idiomes des peuples les plus sauvages, espère pouvoir jeter de loin les bases d'un Dictionnaire comparatif de toutes les langues connues, ouvrage dont De Brosses, Court de Gébelin et, après eux, le célèbre Pallas ont eu l'idée, et qui, s'il est jamais exécuté, ne pourra l'être que par les soins constants de la Société la plus active et la plus laborieuse ${ }^{136}$.

135 Cf. C. DE BROSSES (1709-1777), Traité de la formation mécanique des langues et des principes physiques de l'étymologie, Paris, Saillant, 1765, 2 vol. (réédité en 1801). On doit également à CH. DE BROSSES un ouvrage important en anthropologie, sociologie et histoire des religions: Du Culte des dieux fétiches, Paris, 1760 (réimpression, Paris, Fayard, 1988 (NDE).

136 COURT DE GÉBELIN (1725-1784), érudit français, s' occupa de recherches sur l'antiquité et sur l'ensemble des connaissances humaines; on lui doit notamment une Histoire naturelle de la parole, Paris, 1776. P. S. PALLAS (1741-1811), naturaliste et ethnographe allemand, fit partie de plusieurs expéditions scientifiques en Sibérie, en Russie et en Crimée. Il publia ses relations de voyage: Voyages en différentes province de l'Empire de Russie et dans l'Asie septentrionale, Paris, Lagrande, 1788-1793, 5 vol. (cf. aussi I'édition revue par LAMARCK, Paris, Maradan, 1794) (NDE). 
Il nous reste à développer en peu de mots le but que la Société a indiqué à ceux de ses membres qui se sont spécialement voués à l'étude de la morale et à celle de la législation.

Ces deux branches sont également étendues, elles sont également dignes de fixer l'attention et le zèle des observateurs de l'homme. Il semble même que les recherches de la Société, relatives à cette partie de ses travaux, sont susceptibles d'avoir une utilité plus générale, plus prochaine, et méritent, par conséquent, de l'occuper d'une manière spéciale.

Quand on adresse à I'homme la célèbre inscription Connais-toi toimême, disait Cicéron, ce n'est pas seulement pour lui ouvrir les yeux sur sa petitesse, c'est aussi pour lui faire apercevoir sa grandeur. La Société, qui a adopté pour sa devise la belle inscription Connais-toi toimême, pourrait peut-être aussi adopter en même temps le commentaire qu'en fait le philosophe romain. Par le seul fait de sa formation cette Société a montré d'une manière éclatante qu'elle sait apprécier la grandeur de l'homme; elle le montrera plus évidemment encore en opposant le témoignage de tous les siècles et de tous les pays à ces principes, éversifs de toute morale et de toute législation, qui rabaissent l'homme au niveau de la brute et lui assignent le néant pour dernier asile.

L'homme est d'autant plus capable de s'élever à de hautes connaissances, il est d'autant plus porté à faire de grandes actions qu'il s'estime davantage. En effet, si je ne suis rien de plus que la brute pourquoi consumer mes jours à des études pénibles? Pourquoi tant de recherches qui ne doivent aboutir qu'à me convaincre de la bassesse de ma nature? Si je méconnais les prérogatives de mon esprit et sa céleste origine, pourquoi prendre la peine de le cultiver? Dès que la raison n'est plus que le produit de la matière, et qu'elle est déshéritée dans l'avenir, la vertu n'est plus qu' un vain mot : c'est aux sens à gouverner l'homme.

Des recherches sur les divers systèmes des philosophes anciens et modernes montreraient, $d$ 'une manière palpable, que ceux d'entre eux qui ont méconnu la grandeur de notre nature ont été ceux dont la doctrine a eu les plus funestes résultats, pour la morale publique et particulière... 
[65]

Il serait aisé d'étendre ces recherches et d'opposer aux principes de ces détracteurs de notre nature les principes, bien différents, des philosophes et des législateurs qui, pour rappeler les hommes à leurs devoirs, les ont d'abord rappelés à l'estime d'eux-mêmes.

La Société, en cherchant à relever la dignité humaine, cette belle prérogative qui fut si cruellement méconnue, si insolemment outragée pendant l'affreux régime qui pesa quelque temps sur la France, aura l'avantage de concourir, par la seule influence de ses observations, à l'extinction d'une foule d'abus que ce régime odieux fit naître et que le gouvernement actuel n'a pu parvenir encore à détruire complètement.

Puisse cette Société, aux succès de laquelle, on peut le dire, l'Europe s'intéresse aujourd'hui, remplir par la suite les glorieuses destinées qui semblent l'attendre, et mériter qu'on dise un jour d'elle que sa fondation fut utile tout à la fois à l'avancement de la science et au bonheur des hommes! 
Aux origines de l'anthropologie française. (1994) Édition revue et corrigée par les auteurs, 1993.

\section{Texte 2}

\section{Note instructive sur les recherches à faire relativement aux différences anatomiques des diverses races d'hommes (1799)}

\section{Par Georges Cuvier}

\section{Retour à la table des matières}

Ce texte a été édité par Maurice Girard en pièce annexe de son ouvrage consacré à François Péron: F. Péron, naturaliste, voyageur aux Terres australes, Paris. J.B. Baillière et fils, 1857, pp. 261-269. L'original autographe aurait été retrouvé par le peintre et dessinateur de l'expédition Baudin, Lesueur, dans les papiers de Péron, et confié à Geoffroy Saint-Hilaire.

Il fut republié en 1910 par Georges Hervé dans I'article: « A la recherche d'un manuscrit. Les instructions anthropologiques de $G$. Cuvier pour le voyage du Géographe et du Naturaliste aux terres australes », Revue de l'École d'anthropologie de Paris, Xème année, 1910, pp. 289306. 
Comme les Considérations... de J. - M. de Gérando, la note de Cuvier a été rédigée à l'attention des « naturalistes » de l'expédition du capitaine Baudin. Lors d'une séance de la Société des observateurs de I'homme (manuscrit non daté retrouvé par E.-T Hamy et cité par $G$. Hervé dans l'article dont il est question plus haut ${ }^{137}$ ), Jauffret précise ainsi les objectifs des organisateurs de cette expédition:

"Estimables confrères,

L'existence d'une Société qui consacrera tous ses travaux à l'avancement de la science de l'homme, ne peut manquer de faire époque dans I'histoire de l'esprit [68] humain. Le siècle est arrivé peutêtre où cette science va faire les progrès qu'ont faits à peu près toutes les autres, et cette réunion d'observateurs aura du moins la gloire d'y avoir puissamment concouru.

Pour arriver au but que la Société se propose, elle ne doit laisser échapper aucune occasion de perfectionner l'anthropologie.

Il s'en présente une, et sans doute la Société sentira combien il est avantageux de s'empresser de la saisir.

On dirait qu'un bon génie veut favoriser vos travaux. Un voyage mémorable se prépare. Au milieu du fracas de la guerre, le gouvernement vient de céder aux vouux des amis de la science. Une expédition autour du monde va s'effectuer. C'est le capitaine Baudin qui va lui donner son nom.

Un homme d'un mérite éminent, mais d'une modestie égale à son mérite, a le plus contribué à faire réussir le plan de cette expédition remarquable, qui aura pour but principal d'aller étudier les productions et les moeurs des habitants de la Nouvelle-Hollande [i.e. Australie]. Cet homme précieux, que notre Société possédera bientôt dans son sein [il s'agit de A. -L. Jussieu], et qui, à ce que j'espère, y amènera le capitaine Baudin avant son départ, est l'un des commissaires chargés

137 C'est en tout cas sous la plume de Hervé que l'on apprend qu' Hamy aurait eu entre les mains les archives de la Société des observateurs de l'homme, lesquelles ont depuis disparu. 
par le gouvernement de donner toutes les instructions nécessaires au successeur de Cook et de Bougainville et de La Pérouse.

Il m'a chargé de demander à la Société des observateurs de I'homme des instructions particulières sur les recherches à faire relativement à l'homme physique, intellectuel et moral des diverses contrées qui seront visitées tour à tour par le capitaine Baudin. Combien il sera glorieux pour la Société d'accoutumer les voyageurs à observer I'homme, si peu observé jusqu'ici! Quel avantage pour elle de pouvoir entretenir une correspondance suivie avec ces hommes courageux qui vont affronter tant de dangers pour multiplier nos connaissances!

Je demande que la Société nomme des commissaires pour rédiger promptement les instructions que l'on attend d'elle: je lui propose de nommer pour commissaires les citoyens Hallé, Cuvier, Sicard et de Gérando. »

Pour des raisons, qui n'ont pas été éclaircies, seuls Cuvier et Gérando rédigeront ces instructions.

Dans une matière où les premiers pas ont à peine été tentés, les instructions doivent encore se réduire à un petit nombre. Ce n'est que lorsque les principales bases auront été posées qu'il sera possible de demander aux voyageurs des observations délicates.

On a cru longtemps que les différences qui caractérisent les races de l'espèce humaine se bornent à la couleur de la peau, à la consistance et à la longueur des [69] cheveux. On a longtemps attribué la forme écrasée de la face des Nègres et de quelques autres peuples à la compression mécanique exercée sur les enfants. À peine pensait-on que cette compression portât ses effets jusque sur la charpente osseuse de la tête et Daubenton écrivait encore, vers le milieu de ce siècle, que les crânes de Nègre, de Chinois, de Calmouque, ne lui avaient présenté aucune différence sensible avec les crânes d'Européens. 
Après plusieurs essais infructueux pour apprécier plus exactement les différences, Camper trouva une méthode géométrique qui lui donna des résultats constants. Cette méthode a été perfectionnée depuis: elle a été appliquée à un grand nombre de crânes, et on ne peut plus douter que la proportion du crâne à la face, la saillie du museau, la largeur des pommettes, la forme des orbites ne soient soumises à des proportions peu variables dans chaque race, mais assez différentes d'une race à une autre. L'influence que ces diverses structures peuvent avoir sur les facultés morales et intellectuelles de ces diverses races a été appréciée jusqu'à un certain point, et l'expérience semble assez d'accord avec la théorie dans tout ce qui concerne les rapports entre la perfection de l'esprit et la beauté de la figure.

Blumenbach a recueilli, par une opiniâtreté de recherche très remarquable, une centaine de têtes des diverses nations de la terre. Il en a déjà publié trente; il en a fait des comparaisons soignées qui lui ont donné, d'une manière précise, les limites de la variabilité dans les grandes races de l'ancien continent, la caucasique ou blanche, la mongolique ou jaune, et l'éthiopique ou nègre. Il nous paraît qu'il n'a pas encore autant de certitude sur trois autres, qui se rapprochent peutêtre plus ou moins de l'une ou de l'autre des précédentes, la laponne ou brune, l'américaine ou rouge, et celle des îles de la mer du Sud et de la Nouvelle-Hollande, qui varie du jaune au noir. C'est faute de matériaux, faute d'objets comparables, qu'il reste encore quelque indécision sur ce sujet, et ce n'est qu'en en rassemblant qu' on pourra la lever.

En vain voudrait-on s'en rapporter aux observations faites sur les lieux par des voyageurs et consignées dans leurs journaux. L'expérience prouve qu'en histoire naturelle, toute description absolue est vague, et que toute comparaison faite entre un objet présent et un objet absent est illusoire. Les plus grands naturalistes ont confondu ou distingué mal à propos les êtres les plus faciles à reconnaître, parce qu'ils ne les avaient pas à la fois sous les yeux. Que sera-ce lorsqu'il s'agira de comparer des objets dont les différences sont si délicates et sur lesquels on se trompe, même lorsqu' on les voit à côté l'un de l'autre? Tout le monde sait que les plus grands peintres ont souvent mal saisi le caractère du Nègre et n'ont peint qu'un Blanc barbouillé de suie. 
Les dessins qui se trouvent dans les voyages modernes, quoique faits sur les lieux, se ressentent plus ou moins des règles et des proportions que le dessinateur avait apprises dans les écoles d'Europe, et il n'en est presque aucun sur lequel le naturaliste puisse assez compter pour en faire la base de recherches ultérieures.

[70]

Il faut donc absolument recueillir les pièces anatomiques dans un même local et les y comparer dans tous leurs rapports. C'est là une première base à laquelle on peut rapporter ensuite les observations sur le physique et le moral de chaque race. Les hommes eux-mêmes, rassemblés vivants, seraient sans doute les meilleurs matériaux pour une comparaison exacte des diverses variétés de l'espèce humaine: mais sans parler des difficultés insurmontables d'une réunion de cette nature, il ne nous est pas permis, même lorsque nous le pourrions, de sacrifier le bonheur, ni même de violer les volontés de nos semblables pour satisfaire une simple curiosité philosophique. Des portraits vrais et nombreux et des préparations anatomiques, voilà donc tout ce que nous pouvons espérer des voyageurs. Si ces objets sont accompagnés d'observations faites avec esprit et avec soin sur les lieux, ils suffiront à nos travaux.

Il faut des études particulières pour le genre de portraits que nous exigeons: il doit réunir au mérite des portraits ordinaires celui d'une précision géométrique qu'on ne peut obtenir que dans certaines positions de la tête, mais qui doit être rigoureuse. Ainsi, il faut toujours que le profil pur soit joint au portrait de face. Le choix des individus n'est pas indifférent lorsque cela est possible. Il en faudra prendre de divers âges, de divers sexes et de divers états dans chaque peuple. Les costumes, les marques par lesquelles la plupart des sauvages se défigurent, et que les voyageurs ordinaires ont tant de soin de nous transmettre, ne servent qu'à masquer le véritable caractère de la physionomie. Il serait important que le peintre représentât toutes ses têtes avec le même arrangement des cheveux, le plus simple possible, et surtout celui qui cacherait moins le front et qui altérerait moins la forme du crâne. Tous les ornements étrangers, les bagues, les pendants, le tatouage, doivent être supprimés. Il faut que le dessinateur ait étudié la dissertation célèbre de Camper sur les moyens de rendre 
les caractères des diverses races humaines; quelque imparfaite qu'elle soi, elle lui fournira des idées qui germeront, s'il a l'esprit nécessaire à son art.

Parmi les pièces anatomiques, la principale et la plus nécessaire à obtenir, c'est la tête osseuse. On en a déjà quelques-unes, mais il s'en faut beaucoup que cette collection soit complète. On n'a d'ailleurs presque rien sur les différences d'âge et de sexe dans les diverses nations. Ces objets ne sont pas aussi aisés à se procurer que les portraits, aussi les voyageurs ne doivent-ils négliger aucune occasion lorsqu'ils peuvent visiter les lieux où les morts sont déposés, lorsqu'ils seront témoins de quelque combat ou qu'ils y prendront part. Lorsqu'ils pourront, d'une manière quelconque, disposer d'un cadavre, ils doivent soigneusement noter tout ce qui a rapport à l'individu dont ce cadavre proviendra autant qu'ils pourront le savoir. Des squelettes entiers seraient infiniment précieux. Croirait-on qu'on n'a encore, dans aucun ouvrage, la comparaison détaillée du squelette du Nègre et de celui du Blanc?

La préparation de ces objets ne fera, sans doute, point de difficultés. Faire bouillir les os dans une dissolution de soude ou de potasse caustique et les débarrasser [71] de leurs chairs, c'est l'affaire de quelques heures. Les marins s'opposeront peut-être à ce que ces opérations, qui leur paraissent barbares, s'accomplissent sur le vaisseau : mais dans une expédition qui a pour but l'avancement des sciences, il faut que les chefs ne se laissent gouverner que par la raison et qu'ils sachent l'inspirer à leurs équipages.

Les os décharnés de chaque squelette seront enfermés dans un sac de toile, sans aucun ordre; on les rassemblera en Europe. Chaque tête isolée sera aussi enveloppée, de peur que quelque pièce ne s'en détache On étiquettera chaque paquet. Il serait facile, à présent, de rapporter des têtes en chair qui pourraient être fort utiles pour corriger et perfectionner les dessins; il ne s'agirait que de les mettre dans une dissolution de sublimé corrosif. Après y avoir séjourné quelque temps, on les en retire et on les laisse sécher. Elles deviennent dures comme du bois, en conservant à peu près leurs formes, et sont à jamais inattaquables aux insectes. 
Quoique la collection des objets ci-dessus soit intéressante à faire partout, elle doit l'être avec plus de soin, s'il est possible, dans les lieux dont les habitants ne sont pas encore suffisamment connus. Ainsi les Papous, ou habitants de la Nouvelle-Guinée, que l'on a regardés longtemps comme des Nègres, les habitants de presque toute la Nouvelle-Hollande, ceux des îles de la mer du Sud, les habitants du détroit de Magellan ou Patagons, les Madécasses, doivent surtout attirer l'attention des voyageurs. 
[73]

Aux origines de l'anthropologie française. (1994)

Édition revue et corrigée par les auteurs, 1993.

Texte 3

\title{
Considérations sur les diverses méthodes à suivre dans l'observation des peuples sauvages (1799)
}

\author{
Par Joseph-Marie de Gérando
}

\section{Retour à la table des matières}

Ce mémoire de Joseph-Marie de Gérando a été probablement rédigé en quelques semaines, si ce n'est en quelques jours, au cours de l'été 1799. L'imprimé original, conservé à la bibliothèque du Muséum national d'Histoire naturelle sous la cote M. M. 6 L., porte la mention: Extrait des procès-verbaux des séances de la Société des observateurs de l'homme. Sur la proposition d'un membre, la Société arrête que le mémoire du citoyen Gérando, intitulé: Considérations sur les diverses méthodes à suivre dans l'observation des peuples sauvages, sera imprimé. "Certifié conforme, à Paris, le 28 fructidor an 8 ». Signé Louis-François Jauffret, secrétaire perpétuel de la Société des observateurs de l'homme.

Il a été republié en 1883 par Paul Topinard dans son article «L'Ethnographie en $1800 »$, Revue d'anthropologie, 2 e série, $n^{\circ} 6$, pp. 152-182, puis traduit en anglais et publié par F.T.C Moore, sous le ti- 
tre: The Observation on Savage People, Berkeley, University of California Press, 1969, avec une préface de E. E. Evans-Pritchard.

Le texte original est précédé d'un avertissement que voici :

" Ces Considérations sont adressées au capitaine Baudin, correspondant de la Société, prêt à partir pour son expédition de découvertes, et aux divers observateurs qui l'accompagnent; elles sont adressées aussi au citoyen Levaillant, qui va tenter un troisième voyage dans I'intérieur de l'Afrique. Comme il est possible que [74] les uns et les autres aient occasion de rencontrer des peuples qui appartiennent $a$ des degrés très différents de civilisation ou de barbarie, on a cru qu'il fallait prévoir toutes les hypothèses, et généraliser tellement ces Considérations, qu'elles pussent s'appliquer à toutes les nations qui diffèrent, par leurs formes morales et politiques, des nations de l'Europe. L'on s'est surtout attaché à présenter un cadre complet qui pût réunir tous les points de vue sous lesquels ces nations peuvent être envisagées par le philosophe. On n'a pas pensé qu'il fallût supprimer certaines questions simples et faciles à prévoir, mais qui étaient nécessaires à l'intégrité de l'ensemble. »

Dans le texte, que nous reprenons ici, nous avons conservé la disposition typographique originale, ainsi que la ponctuation.

\section{Importance de l'étude de l'homme en général}

Il semble étonnant que, dans un siècle d'égoïsme, on éprouve tant de peine à persuader à l'homme que de toutes les études, la plus importante est celle de lui-même. C'est que l'égoïsme, comme toutes les passions, est aveugle. L'attention de l'égoïste se concentre dans les besoins immédiats dont ses sens l'avertissent, et ne sait point s'élever jusqu'à ces besoins réfléchis que nous découvre la raison; il cherche à 
se satisfaire et non à se perfectionner. Il ne considère que son individu ; son espèce n'est rien pour lui. Peut-être craint-il, en pénétrant les mystères de son être, de fixer son propre avilissement, de rougir de ses découvertes, et de rencontrer sa conscience.

La vraie philosophie, toujours d'accord avec la morale, nous tient un autre langage. La source des utiles lumières, nous dit-elle, comme celle du solide bonheur, est en nous-mêmes. Nos lumières dépendent surtout de l'état de nos facultés: mais comment perfectionner nos facultés, si nous n'en connaissons la nature et les lois? Les éléments de la félicité sont les sentiments moraux: mais comment développer ces sentiments, si nous n'examinons le principe de nos affections et les moyens de les diriger? C'est en s'étudiant qu'on s'améliore; le sage est celui qui se connaît bien. En méditant ainsi la nature de son être, on sent mieux tous les rapports qui nous unissent à nos semblables, on retrouve dans le fond intime de son existence cette identité de la vie commune qui nous anime tous, on sent toute la force de cette belle maxime des Anciens : « Je suis homme et rien d'humain n'est étranger pour moi ».

[75]

Mais quel est le moyen de bien étudier l'homme? Ici l'histoire de la philosophie, la voix du monde savant nous répondent. Le temps des systèmes est passé. Las de s'être en vain agité, pendant des siècles, en de vaines théories, le génie du savoir s'est enfin fixé sur la route de l'observation. Il a reconnu que le véritable maître est la nature; il a mis tout son art à l'écouter avec soin, à l'interroger quelquefois. La science de I'homme aussi est une science naturelle, une science d'observation, la plus noble de toutes. Eh! quelle science ne serait pas une science naturelle? L'art lui-même, qu'on veut opposer quelquefois à la nature, n'a pour objet que de l'imiter.

L'esprit d'observation a une marche sûre: il rassemble les faits pour les comparer, et les compare pour les mieux connaître. Les sciences naturelles ne sont en quelque sorte qu' une suite de comparaisons. Comme chaque phénomène particulier est ordinairement le résultat de l'action combinée de plusieurs causes, il ne serait pour nous qu'un pro- 
fond mystère, si nous le considérions d'une manière isolée: mais en le rapprochant des phénomènes analogues, ils se renvoient les uns aux autres une mutuelle lumière. L'action spéciale de chaque cause se montre à nous distincte et indépendante, et les lois générales en résultent. On n'observe bien qu'en analysant; or, on analyse en philosophie par les rapprochements, comme en chimie par le jeu des affinités.

L'homme tel qu'il se montre à nous, dans les individus qui nous entourent, se trouve à la fois modifié par mille circonstances diverses, par l'éducation, le climat, les institutions politiques, les moeurs, les opinions établies, par les effets de l'imitation, par l'influence des besoins factices qu'il s'est créés. Au milieu de tant de causes diverses qui se réunissent pour produire ce grand et intéressant effet, nous ne saurons jamais démêler l'action précise qui appartient à chacune si nous ne trouvons des termes de comparaison qui isolent l'homme des circonstances particulière dans lesquelles il s'offre à nous, et qui lui enlèvent ses formes accessoires sous lesquelles l'art a voilé en quelque sorte à nos yeux l'ouvrage de la nature.

\section{De l'observation des sauvages en particulier}

Or, de tous les termes de comparaison que nous pouvons choisir, il n'en est point de plus curieux, de plus fécond en méditations utiles que celui que nous présentent les peuples sauvages. Ici, nous pouvons relever d'abord les variétés qui appartiennent au climat, à l'organisation, aux habitudes de la vie physique, et nous remarquerons que parmi des nations beaucoup moins développées par l'effet des institutions morales, ces variétés naturelles doivent ressortir d'une manière [76] beaucoup plus sensible: moins distinguées entre elles par les circonstances secondaires, elles doivent l'être surtout par ces circonstances premières et fondamentales qui appartiennent au principe même de l'existence. Ici, nous pourrons trouver les matériaux nécessaires pour composer une échelle exacte des divers degrés de civilisation, et pour assigner à chacun les propriétés qui le caractérisent; nous pourrons reconnaître quels sont les besoins, les idées, les habitudes qui se produisent à chaque âge de la société humaine. Ici, le développement des 
passions et des facultés de l'esprit se trouvant beaucoup plus limité, il nous deviendra bien plus facile d'en pénétrer la nature, d'en assigner les lois essentielles. Ici, les générations n'ayant exercé les unes sur les autres qu'une très légère influence, nous nous trouverons en quelque sorte reportés aux premières époques de notre propre histoire: nous pourrons établir de sûres expériences sur l'origine et la génération des idées, sur la formation et les progrès du langage, sur l'enchaînement qui existe entre ces deux ordres d'opérations. Le voyageur philosophe qui navigue vers les extrémités de la terre, traverse en effet la suite des âges, il voyage dans le passé ; chaque pas qu'il fait est un siècle qu'il franchit. Ces îles inconnues auxquelles il atteint, sont pour lui le berceau de la société humaine. Ces peuples que méprise notre ignorante vanité, se découvrent à lui comme d'antiques et majestueux monuments de l'origine des temps: monuments bien plus dignes mille fois de notre admiration et de notre respect que ces pyramides célèbres dont les bords du Nil s'enorgueillissent. Celles-ci n'attestent que la frivole ambition et le pouvoir passager de quelques individus dont le nom même nous est à peine parvenu; ceux-là nous retracent l'état de nos propres ancêtres, et la première histoire du monde.

Et lors même que nous ne verrions pas dans les peuples sauvages un utile objet d'instructions pour nous-mêmes, ne serait-ce pas assez des nobles sentiments de la philanthropie pour nous faire attacher une haute importance aux communications que nous pouvons former avec eux? Quel plus touchant dessein que celui de rétablir ainsi les augustes naiades de la société universelle, que de retrouver ces anciens parents séparés par un long exil du reste de la famille commune, que de leur tendre la main pour s'élever à un état plus heureux! Ô vous, qui, portés par un généreux dévouement sur ces rives lointaines, approcherez bientôt de leurs huttes solitaires, paraissez auprès d'eux comme les députés de l'humanité tout entière! Présentez-leur, en son nom, le pacte d'une fraternelle alliance! Faites-leur oublier que de farouches aventuriers ne cherchèrent leur séjour que pour les dépouiller ou les asservir ; ne vous présentez à eux que pour leur offrir [77] des bienfaits. Portez-leur nos arts, et non notre corruption, le code de notre morale et non l'exemple de nos vices, nos sciences, et non pas notre scepticisme, les avantages de la civilisation, et non pas ses abus ; cachez-leur qu'en ces contrées aussi, quoique plus éclairées, les hommes 
s'entre-déchirent par des combats, et se dégradent par leurs passions. Assis auprès d'eux, au milieu de leurs forêts désertes et sur leurs rivages ignorés, ne leur parlez que de paix, d'union, de travaux utiles; dites-leur que, dans ces empires inconnus d'eux, que vous avez quittés pour les visiter, il est des hommes qui forment des vœux pour leur bonheur, qui les saluent comme des frères, et qui s'associent de toute leur âme aux intentions généreuses qui vous amènent au milieu d'eux.

\section{Vices des observations faites jusqu'à présent}

En vous exprimant ici tout ce que nous attendons de vos soins et de vos efforts, nous n'avons garde de vouloir déprécier les nombreux services rendus à la société par les voyageurs qui vous ont précédés. N'eussent-ils fait que préparer la voie, par leurs hardies tentatives, à ceux qui devaient les suivre, et leur fournir de précieuses indications, ils auraient acquis par cela seul de grands droits à notre reconnaissance. Mais ils ont commencé d'établir quelque correspondance avec les nations sauvages: ils nous ont rapporté divers renseignements sur leurs mours et leur langage. Seulement, partagés par d'autres soins, plus empressés de découvrir de nouveaux pays que de les étudier toujours en mouvement lorsqu'il eût fallu beaucoup de repos, prévenus peut-être de ces préjugés injustes qui flétrissent à nos yeux les nations sauvages, ou du moins, témoins de l'indifférence que notre Europe a pour elles, ils ne se sont pas assez appliqués à nous rapporter d'exactes et complètes remarques; il leur est arrivé, comme il arrive toujours à ceux qui observent avec trop de précipitation et de légèreté, ils ont observé assez mal, et l'imperfection de leurs rapports a été la punition de notre insouciance ${ }^{138}$. Comme l'homme est toujours plus

138 Nous n'avons pas besoin de prévenir que les réflexions critiques, que nous faisons ici sur les relations des voyageurs, ne s'adressent qu'aux relations ordinaires, et reçoivent par conséquent de notables exceptions. Loin de nous de vouloir affaiblir l'admiration qui est due aux Cook, aux Bougainville, etc. À cet égard, votre pensée nous aura prévenus : la méditation de leurs écrits a été votre première étude. (Gérando s'adresse ici, probablement, à Levaillant et à Baudin à qui, nous le rappelons, ces Considérations sont destinées.) 
curieux des nouveautés qui frappent ses sens que des instructions qui s'adressent à sa raison, on a attaché bien plus de prix à rapporter de ces pays inconnus des plantes, des animaux et des substances minérales, que des expériences sur les phénomènes de la pensée. Ainsi, les [78] naturalistes enrichissaient chaque jour leurs cabinets de nombreuses familles, pendant que les philosophes consumaient le temps à disputer vainement dans leurs écoles sur la nature de l'homme, au lieu de se réunir pour l'étudier sur le théâtre de l'univers.

Passons en revue les fautes principales qu'ont commises ces voyageurs dans leurs observations sur l'homme sauvage, et les vides qu'ils ont laissés dans leurs relations. En remarquant ce qu'ils n'ont pas fait, nous apercevrons mieux ce qui reste à faire.

\section{Premier défaut}

Le premier défaut que nous remarquons dans les observations des voyageurs sur les sauvages, c'est qu'elles ne sont point assez complètes, et c'est là ce qu'on devait attendre du peu de séjour qu'ils faisaient au milieu d'eux, des objets qui partageaient leur attention et du manque de tables régulières auxquelles ils rapportassent leurs remarques. Tantôt, se bornant à étudier quelques individus isolés, ils ne nous ont fourni aucune donnée sur leur état de société, et nous ont ôté par là le moyen de juger l'influence que ces rapports pouvaient avoir sur les facultés individuelles. Tantôt s'arrêtant aux moindres circonstances de leur vie physique, ils nous ont à peine transmis quelques détails sur leurs habitudes morales. Tantôt en décrivant les habitudes des hommes faits, ils ont négligé de s'instruire du mode d'éducation qu'ils reçoivent dans l'enfance et la jeunesse; et surtout, frappés presque entièrement du caractère extérieur et le plus sensible d'un peuple, de ses cérémonies, de son costume, ils ont ordinairement pris trop peu de soins pour s'initier aux circonstances bien plus importantes de son existence philosophique, de ses besoins, de ses idées, de ses passions, de ses connaissances, de ses lois. Ils ont décrit des formes, plutôt 
qu'ils n'ont rapporté des instructions; ils ont saisi quelques effets, et n'ont expliqué presque aucune cause.

\section{Second défaut}

Encore ces observations si insuffisantes, n'ont-elles pas toujours été très certaines et très authentiques soit parce qu'elles ont été quelquefois trop particulières, et que les voyageurs ont voulu juger d'une nation par quelques hommes, d'un caractère par quelques actions, soit parce qu'ils se sont confiés quelquefois aux récits et aux témoignages des sauvages qu'ils ont rencontrés, et qui peut-être n'ont pas été bien compris, peut-être n'étaient pas très bien instruits de ce qu'on leur demandait, peut-être enfin n'avaient pas intérêt à dire la vérité, ou du moins à la faire connaitre tout entière.

[79]

\section{Troisième défaut}

Ajoutons que ces observations ont été faites dans un mauvais ordre, et souvent même sans ordre. Ces voyageurs n'avaient pas assez compris qu'il y a entre les instructions qu' on recueille sur l'état et le caractère des nations, un enchaînement naturel, nécessaire à leur exactitude, et que les unes doivent servir souvent de préparation aux autres. Il faut étudier les effets avant de vouloir remonter aux principes ; il faut observer les individus avant de vouloir juger la nation; il faut connaître les rapports domestiques des familles, avant d'examiner les rapports politiques de la société; il faut surtout chercher à bien s'entendre avec les hommes auxquels on s'adresse, avant d'établir certains résultats sur les relations qu' on prétend en recevoir. 


\section{Quatrième défaut}

Souvent les voyageurs ont fait reposer sur des hypothèses, ou fautives, ou tout au moins douteuses, les récits qu'ils nous ont transmis. Rien ne leur est plus ordinaire, par exemple, que de juger les mœurs des sauvages par des analogies tirées de nos propres mœurs, qui ont cependant si peu de rapports avec elles. Ainsi, d'après certaines actions, ils leur attribuent certaines opinions, certains besoins, parce qu'elles résultent ordinairement en nous de ces besoins ou de ces opinions. Ils font raisonner le sauvage à notre manière, lorsque le sauvage ne leur explique pas là ses raisonnements. C'est ainsi qu'ils ont prononcé souvent sur une nation des sentences trop sévères, qu'ils l'ont accusée de cruauté, de vol, de libertinage, d'athéisme. Il eût été plus sage de recueillir un grand nombre de faits, avant de chercher à les expliquer, et de n'admettre les suppositions qu'après avoir épuisé toutes les lumières de l'expérience.

\section{Cinquième défaut}

Il est, par rapport aux relations des voyageurs, une autre cause d'incertitude qui tient bien plus au vice de nos langues, qu'à l'imperfection de leurs remarques: c'est que les termes qu'ils emploient pour nous en transmettre les résultats, n'ont souvent parmi nous que des acceptions vagues et mal déterminées. Par là nous nous trouvons exposés à comprendre dans leurs récits tout autre chose que ce qu'ils ont voulu nous dire. C'est là ce qui arrive, surtout lorsqu'ils veulent nous instruire des opinions qu' un peuple s'est formées par rapport à la religion, à la morale, à la politique. C'est là ce qui arrive encore, lorsqu'au lieu de nous exposer en détail les faits dont ils ont été témoins, avec toutes leurs circonstances, ils se bornent à nous dire sommairement l'impression qu'ils en ont reçue, les jugements généraux qu'ils en ont 
déduits sur le caractère des nations. On eût pu cependant éviter cet inconvénient, si l'on se fût attaché, ou à décrire les choses sans les [80] juger, ou à choisir des expressions dont le sens fût mieux convenu, ou à déterminer avec précision le sens qu'on leur attachait en les employant.

\section{Sixième défaut}

Il est inutile de retracer ici les inexactitudes auxquelles les voyageurs ont été conduits par le défaut d'impartialité, par les préventions qui résultaient de leurs opinions particulières, par les intérêts de leur amour-propre, ou enfin par l'impulsion du ressentiment. Le caractère des hommes estimables qui se dévouent aujourd'hui à cette noble entreprise, nous est une suffisante garantie que de semblables impressions $n$ 'influeront jamais sur leurs rapports. Mais avec les intentions les plus droites et les plus pures, les voyageurs ont été souvent induits en erreur sur le caractère des peuples, par les procédés qu'ils en avaient éprouvés. Ils ont conclu trop légèrement des circonstances de leur réception, au caractère absolu et ordinaire des hommes au milieu desquels ils avaient pénétré. Ils n'ont pas assez réfléchi que leur présence devait être pour eux un sujet naturel de crainte, de défiance et de réserve: que la politique pouvait ajouter beaucoup à ces précautions extraordinaires: que le souvenir d'anciennes incursions pouvait avoir laissé des préventions funestes dans l'esprit de ces peuples: qu'une nation douce et sociable peut se croire cependant dans un état de guerre naturelle avec des étrangers dont les intentions lui sont inconnues, et qu'enfin, pour apprécier sainement le caractère d'une peuplade, il faudrait, en premier lieu, avoir laissé le temps de s'effacer à ces impressions d'étonnement, de terreur, d'inquiétude qui avaient dû d'abord la saisir, et, en second lieu, pouvoir s'initier aux rapports ordinaires que ses membres ont entre eux. 


\section{Septième défaut}

Mais de tous les regrets que nous laissent les récits des voyageurs qui vous ont précédés, les plus vifs sont ceux que nous cause la négligence qu'ils ont mise à nous instruire de la langue des peuples qu'ils ont visités. D'abord le peu de renseignement qu'ils nous ont fournis à cet égard manquent de précision et d'exactitude, soit parce qu'ils ne nous ont point instruits de la manière dont ils s'y sont pris pour interroger les sauvages, soit parce que souvent ils ont pris eux-mêmes peu de soin pour bien poser les questions. Les signes indicateurs et les signes naturels dont ils ont fait usage pour demander aux sauvages les noms des objets, étaient souvent susceptibles par eux-mêmes d'une assez grande incertitude: on ne peut savoir si ceux auxquels on les adressait y attachèrent bien le même sens que les voyageurs qui en faisaient usage, et répondirent ainsi justement à leurs interrogations [81] De plus, pour nous fournir quelques notions utiles et positives sur les idiomes des peuples sauvages, il n'eût pas fallu se borner, comme on l'a fait, à prendre au hasard les noms de diverses choses qui n'ont presque aucun rapport entre elles; il eût fallu suivre du moins une famille d'idées analogues, lorsqu'on ne pouvait noter la langue tout entière, afin de pouvoir porter quelque jugement sur la génération des termes, et sur les liaisons qui existent entre eux; il n'eût pas fallu se contenter de quelques mots détachés: mais il eût été convenable de retenir des phrases entières, afin d'avoir quelque idée de la construction du discours. On eût dû encore chercher à connaître si ces mots étaient simples ou composés, comme leur longueur nous le donnerait souvent à supposer: s'ils se trouvaient déterminés par quelques articles ou quelques particules; enfin s'ils recevaient des inflexions, ou s'ils demeuraient dans l'état absolu, et s'ils étaient soumis à quelque espèce de lois grammaticales. 


\section{Huitième défaut}

Faute de s'être initiés à l'entière connaissance de l'idiome des peuples sauvages, les voyageurs se sont trouvés dans l'impuissance de puiser auprès d'eux les notions les plus curieuses peut-être qu'ils eussent eu à recueillir. Ils n'ont pu nous transmettre les traditions que ces peuples peuvent conserver de leur origine, des révolutions qu'ils ont éprouvée, et des diverses particularités de leur histoire: traditions qui eussent jeté peut-être un grand jour sur l'importante question de savoir comment le globe s'est peuplé, et sur les causes diverses de l'état actuel dans lequel ces nations se trouvent. Ils n'ont pu se faire expliquer l'esprit d'une foule de cérémonies et d'usages, qui ne sont probablement que des allégories; ils nous ont transmis des descriptions bizarres qui amusent l'oiseuse curiosité du vulgaire, mais qui ne fournissent aucune instruction utile à l'esprit du philosophe. Privés de moyens pour lier des entretiens suivis avec ces peuples, ils n' ont pu se former que des idées très hasardées et très vagues de leurs opinions et de leurs idées: enfin, ils n'ont pu nous fournir ces données aussi certaines qu'abondantes que le langage des nations présente sur leur manière de voir et de sentir, et sur les traits les plus secrets et les plus essentiels de leur caractère.

\section{Observations à faire}

\section{$1^{\circ}$ Signes des sauvages}

Le principal objet sur lequel devrait donc se diriger aujourd'hui l'attention et le zèle d'un voyageur vraiment philosophe, serait de recueillir avec soin tous les moyens qui peuvent servir à pénétrer dans la pensée des peuples au milieu desquels il serait placé, et à s'expliquer la suite de leurs actions et de leurs rapports. Ce n'est pas seulement 
[82] parce que cette étude est de toutes la plus importante en ellemême, c'est encore parce qu'elle doit servir de préliminaire et d'introduction à toutes les autres. Comment se flatter de bien observer un peuple qu' on ne sait pas comprendre et avec lequel on ne peut s'entretenir? Le premier moyen pour bien connaître les sauvages est de devenir en quelque sorte comme l'un d'entre eux; et c'est en apprenant leur langue qu' on deviendra leur concitoyen.

Mais s'il y a déjà si peu de bonnes méthodes pour bien apprendre les langues des nations civilisées les plus voisines de nous; si cette étude exige souvent beaucoup de temps et d'efforts, que sera-ce pour apprendre les idiomes des peuplades sauvages dont il n'existe aucun dictionnaire, qui ne peuvent nous être traduits par aucun truchement, qui ne peuvent nous être expliqués, comme les premières par la communauté des habitudes et par la similitude des associations d'idées? $\mathrm{Ne}$ craignons point de le dire; l'art de bien étudier ces langues, s'il pouvait être réduit en précepte, serait un des chef-d'œuvres de la philosophie: il ne peut être que le fruit de longues méditations sur l'origine des idées. Nous nous bornerons ici à quelques aperçus généraux; les réflexions des hommes éclairés auxquels nous les communiquons, et les leçons de l'expérience, en achèveront le développement, en dirigeront I'application.

\section{Langage d'action \\ Diverses espèces de gestes}

Ce qu'il y a de plus important à observer en étudiant les signes des sauvages, c'est l'ordre qu'on met dans cette étude.

Comme la langue articulée des peuples sauvages d'après les données que nous en avons, est composée de signes presque aussi arbitraires et aussi conventionnels que les nôtres, il est évident que, pour établir avec eux une première correspondance, il faut remonter à des signes plus voisins de la nature; il faut commencer avec eux, comme avec les enfants, par le langage d'action. 
Il faut distinguer dans le langage d'action ou les gestes, trois espèces de signes; les signes indicateurs qui se bornent à fixer l'attention sur un objet présent; les signes descriptifs avec lesquels on imite, en l'absence d'un objet, ses formes, son étendue, ses mouvements; enfin les signes métaphoriques avec le secours desquels, lorsqu'on ne peut imiter et peindre un objet, on reproduit au moins les circonstances qui lui sont liées dans nos souvenirs, en rappelant, par exemple, l'effet par sa cause, ou le tout par une de ses parties.

De ces trois espèces de signes, les indicateurs sont ceux dont l'effet est le plus certain et le moins sujet aux équivoques, lorsqu'ils peuvent être employés. Ce sont donc ceux par lesquels on doit commencer : [83] c'est à eux qu' on doit recourir dans toutes les incertitudes. Il ne faut songer à décrire que lorsqu' on ne peut montrer.

Les signes descriptifs seront d'autant plus utiles, que les descriptions seront mieux faites. Or, la bonté des descriptions dépendra de l'art avec lequel on aura su observer les trois conditions suivantes: imiter les propriétés les plus frappantes et les plus sensibles des objets, celles qui leur sont plus spéciales, et qui par là deviennent plus propres à les distinguer de ceux avec lesquels on pourrait les confondre: enfin, celles qui ont dû être plus particulièrement remarquées des individus auxquels on s'adresse, soit par la nature de leurs dispositions, soit par l'effet des circonstances dans lesquelles ils étaient placés.

Les signes métaphoriques sont ceux de tous qui se trouvent le plus sujets à l'incertitude, et qui sont plus difficiles à interpréter avec précision. Cependant on est souvent forcé d'y recourir. Alors, si le voyageur en fait usage, il ne négligera rien de ce qui peut les rendre plus expressifs; il prendra garde de supposer trop légèrement dans la tête des sauvages des associations d'idées analogues à celles qu'il a formées lui-même. Si c'est le sauvage qui les emploie, le voyageur aura soin de recueillir avec fidélité toutes les circonstances qui les accompagnent, et de les rapprocher des habitudes déjà connues de ce peuple, qui peuvent en faciliter l'explication. 


\section{Des gestes des sourds-muets}

On ne saurait trop recommander aux voyageurs pour lesquels ces réflexions sont rédigées, de prendre une connaissance particulière des signes méthodiques que le citoyen Sicard emploie avec tant de succès pour établir ses premières communications avec les sourds-muets. Car le sourd-muet est aussi un sauvage, et la nature est le seul interprète qui puisse lui traduire les premières leçons de ses maîtres. Cependant, il est important d'observer qu' on ne doit pas s'attendre que les gestes employés à l'égard des sourds-muets aient toujours un effet semblable, par rapport aux peuples sauvages; en effet, la valeur de ces signes dépend surtout des habitudes de ceux auxquels on les adresse: ces habitudes, à leur tour, sont en grande partie l'effet des circonstances au milieu desquelles ils sont placés. Or les circonstances qui entourent le sourd-muet élevé au milieu de nous, ne sont pas communes au sauvage qui n'est point sorti de ses forêts. Les voyageurs s'attacheront donc à choisir parmi ces gestes ceux dont l'expression a moins de rapport aux circonstances accidentelles; ils sauront les modifier d'après les habitudes qu'ils doivent supposer aux peuplades sauvages: ils s'attacheront plutôt à saisir la méthode générale des [84] signes employés par l'instituteur des sourds-muets, qu'à répéter scrupuleusement tous les gestes dont il fait usage.

Il serait à désirer qu'on pût recueillir avec soin tous les signes naturels et imitatifs qu'on rencontrera chez les sauvages, et nous en transmettre le tableau avec l'explication précise de l'interprétation qu'il y attachent. 


\section{Langage articulé}

Ordre dans lequel il faut l'apprendre

Lorsque les voyageurs auront ainsi obtenu, par l'emploi du langage d'action, un premier moyen de s'entendre avec les sauvages, ils passeront à l'étude de leur langue articulée.

Ici, l'ordre qu'ils devront suivre est celui qui se trouve le plus conforme à la génération des idées.

Éléments du langage

Idées primitives

Les premiers mots qu'ils chercheront à connaître seront ceux des objets à la fois les plus simples et les plus sensibles, comme ceux des différentes parties du corps et des substances matérielles qui s'offrent aux regards du sauvage.

Noms

Ils devront s'attacher à ne demander d'abord que des noms de choses ou de substances, et non pas ceux des qualités, actions ou rapports, parce que les premiers sont ceux qui présentent moins d'équivoques, et qui éprouvent moins de transformations dans le discours.

\section{Adjectifs}

Ils passeront ensuite à ceux des qualités sensibles, comme les couleurs, les odeurs, les saveurs, la dureté, la mollesse.

\section{Verbes}

De là ils viendront aux noms des actions sensibles, comme marcher, boire, manger, pêcher, etc. dont les idées sont toujours plus com- 
plexes, en évitant d'y associer aucune circonstance de temps, de lieu, de rapport, afin d'avoir les verbes dans leur état absolu.

\section{Prépositions}

Ils finiront par les termes employés à exprimer des rapports, et qui ont donné naissance dans nos langues aux prépositions et adverbes. Comme l'idée d'un rapport naît toujours de la comparaison de deux ou plusieurs objets, ils auront soin de placer ces objets sous les yeux des sauvages, et de les disposer de telle manière, que le rapport dont ils demanderont l'expression, soit celui qui vienne s' offrir plus naturellement à leur attention.

\section{Idées complexes}

Ces premiers éléments étant fixés, ils pourront demander les termes attachés aux idées plus complexes comme celles d'un village, d'une forêt, d'une armée, de la guerre, etc., en prenant soin de spécifier exactement les circonstances essentielles qui serviront de base à ces idées complexes.

\section{Idées abstraites}

Les sauvages ne peuvent sans doute posséder un grand nombre d'idées abstraites, parce qu'ils n'ont pas eu l'occasion d'exécuter des comparaisons systématiques. Cependant, le besoin de simplifier porte [85] si naturellement I'homme à abstraire, même à son insu, et à se composer des idées de genre et d'espèce, que les sauvages ne peuvent en être dépourvus. Pour les interroger à cet égard, il faudra commencer par les abstractions les plus faciles, c'est-à-dire celles qui supposent des comparaisons moins répétées. Ainsi, en leur montrant deux arbres d'espèce différente, on saura bientôt s'ils ont un nom distinct pour chaque espèce, et un pour l'arbre pris en général; de même pour les animaux. En leur faisant remarquer à la fois le mouvement d'un être animé, et celui d'une pierre, on apprendra s'ils ont un nom pour l'idée abstraite du mouvement. A mesure qu'ils satisferont à ces ques- 
tions, on s'élèvera à des abstractions plus éloignées, en leur faisant exécuter des comparaisons plus étendues.

\section{Idées réfléchies}

Les notions dont les sauvages doivent être le moins occupés, sont celles qui appartiennent à la réflexion, et qui sont du ressort de la morale et de la logique, comme celles de la pensée, du jugement, de la volonté, de la douleur, etc. C'est par celles-ci qu' on terminera ; c'est à leur égard qu'on devra apporter plus de soin et de précision: c'est en les interrogeant sur ces idées qu'on devra surtout se tenir en garde contre les habitudes qui tiennent à notre éducation particulière et qu'on devra éviter de prêter aux sauvages les raisonnements de nos philosophes. Il faut tâcher de pénétrer ce qu'ils pensent, et non prétendre les faire penser à notre manière. Les idées morales sur lesquelles on devra les interroger, seront celles qui se lient de plus près aux idées sensibles, comme celles du désir, de l'espérance, de la crainte, de la joie, de la mort, etc. Ces interrogations se feront en décrivant les actions extérieures qui accompagnent ces manières d'être en évitant toutefois qu'ils ne donnent le nom de l'action pour celui de l'impression secrète qui la détermine.

\section{Ensemble des discours}

Ayant ainsi fixé à peu près la nomenclature des termes élémentaires, il sera à propos de se faire prononcer des discours entiers, et de noter avec beaucoup de soin: $1^{\circ} l^{\prime}$ 'ordre dans lequel les mots y seront distribués: $2^{\circ}$ les modifications que ces mots auront pu recevoir dans leur association réciproque: $3^{\circ}$ les termes auxiliaires qui auront été employés à la liaison du discours, et à la fonction précise à laquelle chacun d'eux aura été appliqué.

\section{Questions à résoudre}

Alors on pourra satisfaire aux question suivantes: 
L'idiome de tel peuple sauvage admet-il des mots composés, ou n'a$t$-il que des radicaux?

Ces mots composés suivent-ils dans leur formation la loi de l'analogie?

Les radicaux sont-ils simples et monosyllabiques?

[86]

Ces radicaux ont-ils un caractère marqué d'imitation?

Les noms des objets reçoivent-ils dans le discours quelque modification constante en raison du genre, du nombre et du régime?

Les noms des qualités ou adjectifs se modifient-ils d'après celui des substantifs auxquels ils se rapportent? Ont-ils des comparatifs, des augmentatifs, des diminutifs, etc.?

Les noms des actions ou les verbes, se modifient-ils selon les personnes, les temps, et les rapports du discours, ou demeurent-ils seulement dans l'état absolu?

Y a-t-il des articles et des conjonctions, et quelles sont leurs espèces?

Ce peuple a-t-il quelque idée des lois de la syntaxe?

A-t-il quelque sentiment des propriétés de l'harmonie et des beautés oratoires?

\section{Précautions à prendre}

Ce ne serait pas assez d'observer l'ordre dont nous venons de donner une idée, si on ne s'attachait, dans la pratique, à prendre quelques précautions indispensables pour prévenir toutes les équivoques.

C'est surtout dans la manière d'interroger les sauvages qu'on doit apporter un très grand soin: car ces interrogations ont ordinairement quelque chose de vague, qui les rend susceptibles de plusieurs interprétations différentes. 
Ainsi, en montrant un objet, par exemple, le sauvage peut croire qu'on lui demande ou le nom de cet objet, ou seulement celui de quelqu'une de ses qualités, ou celui de l'usage auquel on le destine, ou celui de sa situation, ou seulement celui de son genre.

Or, c'est en répétant ces interrogations de diverses manières, en différentes circonstances, auprès de différents individus, qu'on parviendra à lever l'incertitude qui accompagne ces réponses.

Lorsque les sauvages répondent par des mots aussi longs que ceux qui nous ont été transmis par Cook, ou même par des mots de plusieurs syllabes, il serait à propos d'essayer, en prononçant chaque syllabe séparément, si les sauvages ne leur attachent pas quelque sens particulier et si ce qu'on a pris pour un seul mot, n'était pas, par conséquent, un terme composé.

Lorsqu' on aura recueilli des discours entiers, on fera bien d'en changer quelquefois l'ordre, et de les altérer en plusieurs manières, par l'addition ou le retranchement de quelque terme; afin d'observer si les effets en sont toujours les mêmes, ou comment ces effets se modifient.

Comme les idiomes des sauvages sont probablement très pauvres, il est inévitable que chaque terme ait pour eux plus d'une acception. [87] On s'attachera à recueillir ces acceptions diverses, afin de juger, d'après l'extension qu'ils donnent aux sens des mots, de l'association qu'ils établissent entre leurs idées, et de l'emploi qu'ils font des analogies.

\section{Numération}

Une des parties de l'idiome des sauvages, qu'il faudrait surtout compléter autant que possible, est celle qui est relative à la numération. Ce ne sera pas assez d'avoir reconnu quelles sont les qualités les plus élevées, auxquelles ils ont donné des noms; il faudra aussi recueillir toute la suite des noms qu'ils ont donnés à la série naturelle des nombres, et les méthodes qu'ils suivent, quand ils veulent exprimer 
des quantités plus élevées que celles auxquelles ils ont donné des noms distincts, si toutefois ils ont en effet quelque méthode à cet égard.

Il conviendra de remarquer s'ils modifient les mots attachés aux idées des quantités, pour en former les noms ordinaux, comme premier, second, dixième, centième, et pour en former des termes concrets, comme douzaine, vingtaine, etc.

\section{Peinture et écriture}

Il est peu de nations sauvages qui n'aient essayé de retracer dans des figures plus ou moins grossières l'image des objets dont elles voulaient conserver le souvenir. Il serait intéressant de remarquer quel est le caractère dominant de ces figures, pour connaître quelles sont aussi dans ces objets les circonstances par lesquelles leur attention a été plus vivement frappée. En visitant des peuples qui ont déjà obtenu un commencement de civilisation, on trouvera à cet égard un fonds plus abondant de recherches; on s'attachera à observer s'ils ont commencé à employer ces peintures comme un moyen de s'entendre; s'ils en ont composé quelque espace de système hiéroglyphique; quel serait l'esprit de ces hiéroglyphes, et leurs principales lois; enfin s'ils ont quelque idée d'une écriture, c'est-à-dire de certaines figures conventionnelles, pour représenter ou les idées, ou le langage; ou si du moins ils soupçonnent l'utilité que nous en retirons.

\section{Emblèmes, allégories et signaux}

Il paraît au moins que toutes les nations ont eu recours à certains emblèmes, fondés quelquefois sur des conventions expresses, quelquefois sur des analogies plus ou moins prochaines, pour suppléer à de longs discours. Tels sont les signes de feu ou de guerre, de rupture ou d'alliance : elles y ont joint des allégories, comme diverses espèces de trophées, les cérémonies et les pratiques civiles ou religieuses, [88] les danses surtout, ces danses quelquefois si mystérieuses pour les étrangers, et peut-être si propres à nous instruire de l'histoire des 
nations, ou de leurs caractères; enfin ces nations doivent avoir certains signaux pour s'avertir à de grandes distances, pendant leurs chasses, leurs voyages, ou leurs combats. Les voyageurs ne se borneront plus à la simple description de toutes ces conventions: mais ils chercheront à obtenir, autant qu'il sera possible, l'intelligence du sens qu' on y attache, des effets qu'elles propulsent, et de l'origine qu'elles ont eue.

\section{$2^{\circ}$ État des sauvages et d'abord de l'individu}

\section{Son existence physique}

Quoique cette longue et difficile étude des signes employés par les sauvages, ne soit pas sans doute nécessaire dans son entier pour l'observation de leur état politique et moral, quoiqu'il ne faille pas attendre de l'avoir terminée, pour commencer l'observation des autres objets, les progrès que les voyageurs auront faits sous ce premier rapport, leur donneront les moyens les plus rapides et les plus sûrs pour s'instruire à l'égard du second.

On n'attend pas ici de nous que nous entrions dans un détail approfondi des procédés qu'il faut suivre pour obtenir toutes les connaissances relatives à l'état des peuples sauvages. Le bon esprit des voyageurs, l'expérience qu'ils auront acquise, leur en diront plus, à cet égard, que nos vagues aperçus. Nous nous bornerons donc à leur présenter un tableau sommaire des points les plus importants, sur lesquels ils doivent diriger leur observation, à leur soumettre l'ordre qui nous paraît le plus convenable, et à leur rappeler que les résultats qu'ils nous transmettront, seront plus précieux et plus utiles, à proportion qu'ils seront plus complets.

Toutes ces observations peuvent être rangées sous deux titres principaux : l'état de l'individu et celui de la société.

Les premiers objets à remarquer à l'égard de l'individu sont les circonstances de son existence physique. 
Climat

Telle est d'abord la nature du climat qu'il habite. On ne se contentera pas d'observer les degrés du froid ou du chaud; mais on cherchera à s'assurer des propriétés de l'air qu'il respire, à déterminer son élasticité, sa pureté, sa condensation, son humidité, etc.

\section{Aliments}

Telle est ensuite la qualité et la quantité de ses aliments ordinaires et de ses boissons. Il serait à propos d'analyser avec soin l'eau dont il s'abreuve, et de savoir quelle est sa répugnance à user de nos aliments.

[89]

\section{Forces et actions physiques}

On nous donnera des résultats plus positifs sur les forces physiques de l'individu sauvage ${ }^{139}$. On déterminera quels sont les fardeaux qu'il est capable de lever, de porter ou de traîner: quels sont les mouvements musculaires qu'il exécute avec le plus de succès: quelle est la célérité de sa course: quel est l'espace qu'il est capable de parcourir sans repos; quelle est son habileté à la nage; quels sont les exercices ordinaires auxquels il se livre: on remarquera comment il grimpe sur les arbres, franchit les fossés, gravit les rochers, etc.

Lorsque nous disons: I'individu sauvage, le sauvage, le peuple sauvage, on comprend que nous n'avons pas l'idée de parler du sauvage en général, ni de rappeler tous les peuples sauvages à un même type commun, ce qui serait absurde. Ce n'est ici qu'une expression abrégée pour dire l'individu sauvage, ou le peuple sauvage, auprès duquel le voyageur se trouvera placé dans le moment de ses observations. 
Repos

On nous dira combien d'heures il accorde au repos; si son sommeil est profond; s'il est tranquille, ou s'il paraît occupé par des songes: quel peut être le caractère de ces songes; s'il a une heure fixe pour le sommeil ; s'il supporte la veille sans déplaisir et sans incommodité : quelle est son attitude dans le sommeil ou le repos.

\section{Besoins}

Quelle est dans le sauvage l'intensité de la faim, de la soif, et de la fatigue: quels sont les effets que déterminent ces besoins; s'il a du penchant à l'oisiveté, ou s'il se plaît dans le mouvement.

\section{Anthropophagie}

Des voyageurs philosophes étudieront avec soin l'affreux phénomène de l'anthropophagie, et peut-être nous fourniront-ils quelques moyens, sinon de justifier, au moins d'excuser les erreurs de l'espèce humaine. Ils constateront si les peuples anthropophages ne mangent jamais que leurs ennemis vaincus à la guerre: s'ils joignent à cette action quelques autres circonstance cruelles, et s'ils y attachent quelques idées de vengeance: s'ils paraissent beaucoup redouter le même sort: s'ils l'accompagnent de quelques cérémonies régulières: s'ils ont quelque répugnance à manger la chair de leurs amis, des étrangers : s'ils croient par là faire éprouver quelque souffrance ou quelque honte à l'âme de celui qu'ils ont massacré.

\section{Vêtements}

Les vêtements des sauvages sont ordinairement très bien décrits par des voyageurs: c'est là presque toujours leur principale observation, souvent la seule: mais il faudrait ne pas se borner à remarquer leur costume; il faudrait savoir quelle répugnance ils auraient à en changer, ou à adopter les nôtres; s'il y a quelques raisons qui les y attachent, quelque circonstance particulière qui les leur ait fait adopter, 
car I'habitude et l'imitation ne faisant que confirmer ce qui existe, il faut bien qu'il y ait une première origine de ces usages. Ainsi le besoin de se garantir des insectes, ou des effets du froid et du chaud, [90] peut contribuer beaucoup à la coutume que certains peuples sauvages ont de s'enduire le corps. Il faudrait observer enfin si les costumes varient entre eux à raison de l'âge ou de la prééminence, et s'ils y trouvent un signe d'autorité ou de richesse, quelles idées ils ont de la parure, quelle importance ils y attachent.

\section{Effets moraux des maladies}

Nous demanderons aux voyageurs de nous instruire des effets moraux qui peuvent accompagner les maladies des sauvages; de nous dire s'ils ressemblent à ceux qui ont lieu parmi nous: en quoi ils en diffèrent s'ils supportent la souffrance avec calme, courage et patience où ils puisent les moyens de la supporter, si c'est dans l'apathie du caractère, dans une ignorance de l'avenir qui ne leur laisse pas craindre la durée de la douleur, ou enfin dans quelque idée réfléchie, comme une sorte de gloire et de vanité attachée à tolérer en paix ce qu'ils souffrent, comme parmi les sauvages de l'Amérique. Nous leur demanderons à quel point leurs facultés morales sont altérées par ces dérangements physiques: s'ils sont alors plus accessibles à la crainte, à la crédulité : s'ils appréhendent la mort, et quel peut être le principe de leur appréhension ou de leur sécurité à cet égard.

\section{Imbécillité}

Est-il bien vrai qu' on ne rencontre jamais chez les sauvages aucun exemple du phénomène de la manie, ou bien les exemples en sont-ils seulement très rares? S'il en existe quelqu'un, il est important de recueillir toutes les circonstances des effets comme des causes, afin de les comparer à celles que nous remarquons à l'égard de l'homme civilisé. Du moins, il est à présumer qu'on doit rencontrer des exemples d'imbécillité, état qui dépend beaucoup moins que le précédent des passions de l'âme, et des impressions morales, et qui a presque toujours son principe dans un vice naturel des organes. Alors on aura soin de fixer le degré de cette imbécillité, les caractères extérieurs 
qui l'accompagnent. On recherchera l'occasion première qui aura paru la déterminer : on en distinguera les diverses espèces, s'il s'en offrait plusieurs. On observera en particulier si cette imbécillité se manifeste dans la décrépitude de la vieillesse, à quel âge elle se manifeste, quelle est sa généralité, à quel point elle se fait remarquer, etc.

\section{Éducation physique}

L'éducation physique que les sauvages donnent à leurs enfants est un des points à l'égard desquels les voyageurs nous ont laissés dans une plus profonde ignorance. Peut-être est-ce qu'en effet ces peuples ne prennent aucun soin du développement de leurs enfants, et les abandonnent entièrement à la nature. Mais alors on eût dû nous apprendre, du moins, quels sont les effets qui en résultent, si les enfants sont plus ou moins sujets aux maladies, s'ils entrent plus promptement en possession de leurs forces, si la mortalité est plus ou [91] moins grande parmi eux, quels sont les instincts dominants qu' on remarque chez ces petits êtres, etc. Que si, comme il paraît probable, leurs parents en prennent cependant quelque soin pendant les premières années de leur vie, nous espérons qu'on nous transmettra à cet égard quelques détails: qu'on nous dira quelles sont les précautions que la mère prend à l'égard du nouveau-né ; quelle est l'époque à laquelle elle cesse de l'allaiter: quelle est la première nourriture qu'elle lui présente: quelle est la situation dans laquelle elle le couche; le moment où elle commence à l'abandonner à lui-même: I'usage qu'elle fait des lotions: si elle cherche à le défendre du froid et du chaud; si elle lui donne des secours dans ses maladies; enfin, quels sont les premiers exercices auxquels les enfants sont appliqués: quelles sont leurs occupations dans la vie domestique, les travaux qu'on leur impose, le zèle qu'ils y portent, et le succès qu'ils y obtiennent.

\section{Longévité}

La durée de la vie des sauvages fournira la matière à plusieurs questions. Vivent-ils à peu près également, ou règne-t-il une différence sensible entre la durée de leurs vies, et jusqu'où s'étend cette dif- 
férence? Quelle est à peu près parmi eux la durée de la vie moyenne? Jusqu' où atteignent les exemples de la plus grande longévité ? Quelles sont parmi eux les causes de mort les plus fréquentes?

\section{L'individu considéré comme être moral et intellectuel}

Toutes ces observations qui portent sur les circonstances extérieures de la vie sont assez faciles à recueillir, si les voyageurs ont le loisir de séjourner quelque temps parmi les sauvages, et $s$ 'ils peuvent s'approcher d'eux familièrement. Mais si, après avoir cherché à étudier I'homme physique, ils s'attachent à observer l'homme intellectuel et moral, c'est ici qu'ils rencontreront de nombreuses difficultés, qu'ils auront besoin de se tenir en garde contre les inductions trop précipitées, contre les remarques trop superficielles, contre les préjugés qui résultent de nos habitudes: c'est ici qu'ils devront surtout porter dans leurs jugements la plus sévère défiance, et dans leurs récits la plus grande clarté, et la plus rigoureuse précision.

\section{Sensations}

Le premier objet sur lequel s'arrêteront leurs regards seront les sens de l'homme sauvage. Ils descendront dans le détail des sensations diverses, et ils s'attacheront surtout à la solution des quatre questions suivantes: $1^{\circ}$ Quels sont les sens qui sont en eux les plus exercés, les plus actifs, les plus subtils? $2^{\circ}$ Quelles sont les circonstances qui peuvent avoir déterminé en eux le développement plus marqué d'un sens particulier? $3^{\circ}$ Quel est le degré de développement de chacun de leurs sens comparé à celui qu'on rencontre ordinairement [92] parmi nous ? $4^{\circ}$ Quelle est la classe et l'espèce de sensations à laquelle ils attachent le plus de plaisir?

Le développement d'un sens en particulier doit s'estimer par la réunion de plusieurs choses : $1^{\circ}$ I'art avec lequel on distingue deux ou plusieurs sensations entre elles: $2^{\circ}$ la ténuité des sensations qu'on est capable de remarquer: $3^{\circ}$ le nombre des sensations qu' on peut em- 
brasser simultanément: $4^{\circ}$ la rapidité avec laquelle les opérations sont exécutées: $5^{\circ}$ la faculté que l'on a de les prolonger plus ou moins longtemps sans en être fatigué: $6^{\circ}$ enfin l'exactitude des jugements qui les accompagnent quelquefois. $C^{\prime}$ est ce qui compose, par exemple, ce

que nous appelons la justesse du coup d'œil, et l'art d'apprécier les distances.

Les deux sens dont les observateurs étudieront l'état avec le plus de soin, seront celui du tact et celui de la vue, comme les plus importants de tous. Ils s'attacheront à remarquer si l'état naturel des organes ne contribue pas, en même temps que l'exercice, à la perfection qu'on remarque souvent dans les sens de l'homme sauvage; ils s'informeront si la cécité et la surdité sont plus ou moins fréquentes que parmi nous; et ils ne manqueront pas de recueillir les effets moraux attachés à ces deux états, c'est-à-dire le degré de tristesse, d'impuissance, d'oisiveté qui peut les accompagner, et les moyens que ceux qui sont privés d'un organe prennent pour suppléer à son usage.

\section{Idées. Leur nature}

Nos idées ne sont que nos sensations élaborées. Après avoir donc en quelque sorte enregistré les matériaux sur lesquels le sauvage opère, l'observateur cherchera à connaître quelle est la transformation qu'il leur fait subir. Or, les sensations se transforment de deux manières, par les combinaisons et par les abstractions. En suivant cette double trace, on découvrira quelle est l'étendue précise de la sphère d'idées qui appartiennent à l'individu sauvage, et les limites qui la terminent ; on nous apprendra jusqu'où ils ont porté l'art de généraliser, quelles excursions leur esprit a pu faire dans la région des notions morales : on pourra résoudre ces importantes questions:

Le sauvage a-t-il l'idée d'un principe de sentiment et d'action placé en lui-même?

Comment le conçoit-il ?

A-t-il l'idée de quelque chose de simple et d'invisible, par conséquent d'immatériel? 
A-t-il formé les abstractions de l'être et du néant, du fini et de l'infini, de la durée et de l'éternité, du possible et du nécessaire?

A-t-il quelque idée du beau, et le conçoit-il autrement que comme ce qui lui plaît à lui-même?

[93]

A-t-il quelque idée du bon, et le conçoit-il autrement que comme ce qui lui est utile?

Lui arrive-t-il d'imaginer ce qu'il n'a point vu? A-t-il l'idée d'un autre pays que le sien, d'un autre univers que celui qu'il habite? Etc. Etc.

\section{Leur génération}

Sans doute les observateurs ne croiront pas avoir résolu ces grands problèmes, s'ils se sont bornés à questionner le sauvage, et à en recevoir en réponse ou un signe ou un terme vague. Mais ils sentiront le besoin de soumettre son esprit à une plus difficile épreuve. Ils ne le supposeront parvenu à un certain degré d'abstraction, qu'autant qu'ils lui auront vu exécuter les comparaisons qu'il exige; ils ne le supposeront en possession d'une certaine combinaison, qu'autant qu'il sera capable d'en rendre compte. Lorsqu'ils découvriront dans le sauvage quelque idée générale ou réfléchie, ils s'efforceront de découvrir les circonstances dans lesquelles il les aura obtenues; ils tâcheront d'arracher en quelque sorte au sauvage le secret de son histoire intellectuelle, et de nous transmettre le journal de la génération de ses idées.

\section{Leur liaison}

La grande loi de la liaison des idées est une des principales bases sur lesquelles repose le système intellectuel de l'homme. Ainsi, après avoir reconnu la nature des idées dont le sauvage est pourvu, l'observateur étudiera comment elles s'associent entre elles. Ici il aura trois choses à remarquer: $1^{\circ}$ quelle est la facilité avec laquelle ces liaisons se forment : $2^{\circ}$ quelle est la solidité qu'elles conservent, et la promptitude avec laquelle elles se réveillent: $3^{\circ}$ quelle est l'étendue dont elles jouissent. Comme les besoins sont autant de centres auxquels les 
liaisons d'idées se rapportent, c'est surtout en s'attachant aux besoins que l'observateur découvrira jusqu'où les liaisons d'idées peuvent s'étendre.

\section{Opinions et jugements}

Comment le sauvage porte-t-il un jugement sur les objets qui ne sont pas au moment où il juge, à la portée de ses sens? Est-ce uniquement par l'effet de l'habitude, c'est-à-dire du penchant qui le porte à croire que les choses doivent toujours se répéter de la même manière qu'elles ont eu lieu? Fait-il usage des inductions de l'analogie, et quelle étendue donne-t-il à cette espèce de jugements? Remarque-t-on en lui quelque espèce d'instinct semblable à celui qu'on observe chez les animaux, c'est-à-dire une disposition à faire ce qui lui est utile, ou à éviter ce qui lui est nuisible, lors même que l'expérience n'a pu l'instruire des effets qui doivent en résulter? Quelle est l'influence que l'imitation exerce sur ses jugements et ses actions?

[94]

Dieu

Esprits

Quelle est l'impression que fait sur le sauvage le spectacle des phénomènes ordinaires de la nature? Remonte-t-il de la connaissance des effets à la supposition de certaines causes, et comment imagine-til ces causes? Admet-il une cause première? Lui attribue-t-il l'intelligence, la puissance, la sagesse et la bonté ? La croit-il immatérielle? Lui suppose-t-il un séjour fixe? Lui prête-t-il des agents physiques? La considère-t-il comme une providence, c'est-à-dire comme un être qui veille sur lui et sur la nature? La croit-il éternelle? Lui reconnaîtil un pouvoir sans bornes, ou quelles bornes lui fixe-t-il ? La suppose-til capable de l'entendre, de pénétrer dans sa pensée, de se laisser fléchir par ses prières? Admet-il plusieurs de ces causes? Leur prête-til un pouvoir égal ? Les suppose-t-il d'accord entre elles? Par quelles attributions les distingue-t-il ? Place-t-il entre la cause première et lui des agents invisibles et secondaires? Quelles idées s'en forme-t-il? Accorde-t-il un principe d'action et de sentiment aux astres, aux plantes, aux éléments, etc. ? Quelle idée se forme-t-il des animaux? 


\section{Idées sur son existence}

Immortalité

Quelle impression fait sur le sauvage le spectacle des phénomènes extraordinaires de la nature? Comment se les explique-t-il ? Imaginet-il pour lui-même quelque espèce d'existence antérieure à son apparition sur la terre? A-t-il l'idée de quelque fin pour laquelle il se croit placé dans le monde? Comment conçoit-il la mort? Que suppose-t-il au-delà de la mort? Pense-t-il changer d'existence et de séjour? Croit-il à une existence immatérielle? Fixe-t-il quelque terme à cette existence, et admet-il une époque où il rentre dans le néant? Cette idée lui inspire-t-elle quelque répugnance? Attache-t-il à la vie future quelque idée de punition ou de récompense? Sur quoi fonde-t-il ces idées? Se regarde-t-il comme libre, c'est-à-dire comme capable de choisir à son gré parmi les diverses actions qui se présentent à lui ? ou bien admet-il quelque notion de fatalité?

Facultés. Imagination

Attention

Mémoire

Prévoyance

Réflexion

Les facultés sont à l'entendement ce que les forces sont au corps. Les unes comme les autres se développent par l'exercice, et s'estiment par leurs effets. Ainsi le degré de vivacité et d'énergie dont jouit l'imagination d'un individu, s'estimera par la promptitude avec laquelle il prend ses résolutions, par l'accès qu'il donne à la crainte ou à l'espérance, par l'art avec lequel il sait trouver de nouveaux moyens pour arriver à ses fins, enfin par le penchant qu'il a à décrire, à peindre ce qu'il conçoit ou ce qu'il éprouve. L'imagination est la première faculté qu'on devra étudier chez le sauvage, parce que c'est celle qui 
fournit l'aliment à toutes les autres. L'imagination est toujours la première faculté qui se développe dans l'individu; ainsi le développement de cette faculté sera le signe le plus facile pour reconnaître [95] le degré que cet individu occupe dans l'échelle du perfectionnement intellectuel. L'attention vient ensuite. On nous dira de quel degré d'attention le sauvage est capable: quels sont les motifs qui dirigent en lui cette faculté, les objets qu'elle lui fait plus particulièrement remarquer: c'est en suivant la chaîne de ses besoins qu'on pourra établir ces observations avec une exactitude convenable. La mémoire est centrée sur l'attention: car on ne se rappelle que ce qu' on a suffisamment remarqué. Ainsi l'étude qu' on fera de l'état de ces deux facultés chez le sauvage, se trouvera étroitement liée. On nous dira s'il retient facilement ce qu'il a vu, entendu ou éprouvé ; s'il en conserve longtemps la trace: à quelle époque remontent ses souvenirs; dans quel ordre ils se conservent pour lui ; quels vides ils laissent entre eux: à quels pivots se rattachent pour lui les chaînes qu'ils forment, etc. La prévoyance à son tour doit naître de la mémoire, car prévoir, ce n'est qu'appliquer l'expérience du passé. On remarquera donc quel est l'usage que le sauvage sait faire de l'expérience qu'il a acquise, jusqu'où son coup d'œil s'étend dans l'avenir, comment il tire parti des circonstances où il se trouve, comment il sait se précautionner contre les événements, comment il apprend à corriger ses propres erreurs lorsqu'il rencontre quelque mécompte dans sa conduite. Enfin de toutes les facultés, celle qui se développe le plus facilement, celle qui semble appartenir plus en propre à l'homme civilisé, c'est la réflexion, c'est-à-dire cette faculté en vertu de laquelle nous nous replions sur nous-mêmes pour nous rendre compte de nos sentiments, de nos pensées, et pour pénétrer dans les plus intimes secrets de notre manière d'être. Il serait intéressant de savoir si le sauvage ne possède pas du moins quelque commencement d'une si noble puissance, ou s'il demeure toujours étranger à lui-même : il faudrait observer si, lorsque son activité n'est pas attirée au-dehors par les objets qui l'entourent, et qui sont en rapport avec ses besoins, il retombe alors dans un entier assoupissement, et dans une sorte de végétation, ou s'il ne jouit pas en quelque manière de sa propre existence. Au reste, la preuve la plus sûre qu'on pourra obtenir à l'égard du degré de réflexion dont jouissent les sauvages sera dans le caractère de leur langue. 


\section{Idées réfléchies}

Avec la formation des idées complexes et abstraites, avec le jeu des facultés intellectuelles il se développe dans l'homme un second ordre de besoins que nous appelons réfléchies, parce qu'ils ne se lient pas immédiatement à l'existence. L'observateur cherchera à définir avec précision quelle est la nature et l'étendue de ces besoins chez le sauvage. Il nous apprendra quel est le degré de curiosité auquel il [96] paraît sensible, quels sont les effets que produit sur lui la surprise, à quel point il est accessible à la crainte, ou tourmenté par l'incertitude; il nous dira quel est l'attrait qu'il éprouve pour l'amusement ou le plaisir, s'il recherche les émotions fortes et les sensations variées; il nous dira quel est l'empire qu'il exerce sur lui-même, l'intrépidité ou la faiblesse qu'il témoigne à l'aspect du danger, la confiance ou la présomption que lui inspirent ses succès, la fierté ou la honte que lui donne le retour qu'il fait sur lui-même, les regrets qu'il peut ressentir des actions qui ne lui ont pas réussi, la jouissance qu'il peut goûter dans le sentiment de ses propres forces, l'idée qu'il peut avoir de son infériorité et d'un développement plus heureux que celui qu'il a reçu, etc.

\section{Variétés}

Il n'est pas nécessaire sans doute de prévenir les observateurs qu'ils ne doivent pas se borner à établir des recherches sur un seul individu, mais qu'il est nécessaire de les répéter sur un grand nombre, et de comparer les résultats qui en naîtront. Les voyageurs ne nous présentent ordinairement dans chaque pays qu' un type simple et commun, auquel ils supposent que se rapporte à la fois une peuplade entière. N'y a-t-il donc aucune variété entre les divers membres d'une société sauvage? Cette variété, quoique bien moins sensible, sans doute, que celle qui se présente dans les sociétés civilisées, n'est-elle pas cependant réelle et intéressante à connaître? En étudiant avec plus de soin ces êtres si éloignés de nous, ne remarquera-t-on pas dans les facultés, les habitudes, les idées, les opinions, les penchants, des dif- 
férences produites par l'âge, le sexe, l'organisation, les circonstances? Là aussi, le jeune homme ne doit-il pas être plus impétueux et plus actif, la femme plus timide et plus réservée, le vieillard plus prudent? Là aussi, le tempérament ne peut-il pas inspirer des passions plus vives ou des dispositions plus douces? L'état des organes ne peut-il pas à lui seul donner à un homme une supériorité marquée sur un autre homme? Le genre de vie est-il absolument le même, et chacun ne doit-il pas trouver dans son expérience particulière, ou l'occasion de quelque instruction, ou la source de quelque besoin qui lui est propre?

\section{Le sauvage dans la société}

Après avoir observé l'individu tel qu'il est en lui-même, on le suivra dans les rapports qu'il forme avec ses semblables et ici se présentera un nouvel ordre de recherches.

\section{Vie solitaire}

Nous avons eu, en Europe, plusieurs exemples d'individus trouvés au milieu des forêts, et qui paraissaient $n$ 'avoir eu presque aucune communication avec d'autres hommes. Mais de semblables exemples [97] ne peuvent nous représenter l'état sauvage. C'est le dernier degré de I'humanité. Il est probable qu'il n'y a aucune espèce de sauvages chez lesquels on ne trouve du moins quelque commencement de société. Cependant les voyageurs devront examiner $s$ 'il ne se rencontre pas au milieu d'eux quelques individus que le hasard ait condamnés à une vie entièrement solitaire et, dans ce cas, ils observeront avec soin toutes les particularités dont ils offriront le sujet.

\section{Société domestique}

La première société à laquelle l'homme se trouve appelé par la voix de la nature et par l'impulsion des besoins réciproques, est la société domestique, c'est-à-dire celle qui est composée de la famille. 


\section{Autorité des pères}

Les voyageurs observeront si cette première réunion offre l'image d'une société régulière, et s'il existe quelque subordination entre ses membres. Le père y exerce-t-il quelque autorité ? Quelle est l'étendue, la durée, les effets de cette autorité? Sur quel principe paraîtelle fondée, et quelle idée s'en forment celui qui l'exerce et ceux qui lui obéissent? Quel respect les jeunes gens ont-ils pour les vieillards, et de quelle manière le témoignent-ils? Quel degré de reconnaissance les enfants conservent-ils pour les auteurs de leurs jours?

\section{Parenté et fraternité}

Quelle est la force et le caractère de la liaison qui existe entre les frères? L'âge établit-il entre eux quelque prééminence? Jusqu' où les rapports de parenté s'étendent-ils et conservent-ils quelque influence? Quels sont les égards qu'ils entraînent? Les membres d'une même famille se réunissent-ils pour le travail, la chasse, la nourriture? Quelle loi, quel ordre règnent alors entre eux? Chaque individu est-il libre de se retirer à volonté, ou quels liens le retiennent?

\section{Femmes. Leur état}

L'état domestique des femmes peut être envisagé sous plusieurs rapports. Le premier qui se présente est celui de leur dépendance, ou de la considération qu'on a pour elles. A cet égard, les sauvages ont présenté quelquefois des spectacles assez opposés. En général, cependant, il paraît que les égards pour le sexe sont un effet de la civilisation. Les observations des voyageurs nous apprendront jusqu'à quel point cette idée peut être fondée; elles nous apprendront quels droits on accorde à la femme sur la propriété commune, quels travaux on lui impose, quelle protection on lui assure; enfin elles nous apprendront si, dans les pays même les plus sauvages, le sexe ne conserve pas quelque chose de ce secret et doux empire, fondé à la fois sur sa faiblesse, sa sensibilité et ses charmes. 


\section{Pudeur}

Le second rapport sous lequel les femmes se présentent, est leur conduite à l'égard des lois de la pudeur. Ici, il faut bien observer deux choses : la connaissance des lois, et la fidélité à les observer. Y a-t-il, en effet, un tel degré d'abrutissement chez quelques hordes sauvages, [98] que les femmes n'aient absolument aucun sentiment de pudeur, qu'elles ne se prescrivent aucune réserve, et qu'elles aillent sans rougir au-devant des hommes? Ou bien possédant à cet égard quelques idées de devoir, ont-elles seulement une grande facilité à y manquer, par l'effet du tempérament, ou des occasions, ou de l'imitation, ou des instances?

Amour

Ceci conduit à quelques questions sur l'amour et le mariage.

L'histoire de l'amour chez les peuples sauvages présenterait un tableau aussi curieux qu'intéressant. Son origine, son caractère, ses signes, ses effets, ses sacrifices, ses vengeances, combien tout cela ne doit-il pas différer de ce qui se passe au milieu de nous? Mais ce parallèle est-il à leur avantage ou au nôtre? Le sentiment de l'amour chez les sauvages est-il purement physique? N'admet-il aucune idée de confiance, de privilège, de dévouement, d'association morale? Cesse-t-il avec la jouissance, ou de quelle durée est-il susceptible? Se fixe-t-il sur un seul individu, ou se dirige-t-il indistinctement sur plusieurs, et alors ne porte-t-il avec lui aucune idée d'infidélité et d'inconstance? Les faveurs des femmes sont-elles considérées uniquement comme la récompense de l'amour, et quel prix les hommes y attachent-ils? Quels sont les égards réciproques qui accompagnent le sentiment? L'homme en fait-il ordinairement les avances? Éprouve-t-il souvent des rigueurs? 


\section{Mariage}

Les idées de mariage, c'est-à-dire d'une union légitime entre I'homme et la femme ne s'établissent que dans une société qui a déjà reçu quelque développement. Mais le point où commence un semblable établissement est très important à remarquer. Il ne l'est pas moins d'en fixer toutes les circonstances. Le mariage est-il considéré seulement comme le résultat du libre consentement des deux époux? La volonté des parents suffit-elle pour contraindre les enfants à s'unir? Ou bien faut-il la réunion de ces deux choses? Le mariage est-il considéré comme un acte civil auquel la société soit intéressée, et alors quelle part y prend-elle? Le mariage est-il considéré comme un acte religieux, et alors sous quel rapport les idées religieuses y concourent-elles?

\section{Son caractère, ses effets}

Quels devoirs réciproques s'établissent entre les époux, et quelle en est la garantie? La paternité ajoute-t-elle beaucoup à leurs liens? Comment la vieillesse les modifie-t-elle? Est-ce un usage parmi les peuples sauvages, que celui de faire cesser toute communication avec le sexe féminin, pendant l'époque de ses incommodités ordinaires?

Divorce, polygamie

Y a-t-il quelque exemple chez les sauvages de l'indissolubilité du mariage, et sur quel principe alors paraît-elle fondée? Parmi ceux qui [99] divorcent, il faudrait savoir quel est le nombre et la fréquence des divorces, les motifs qui les déterminent, les formes qui les accompagnent, les effets qu'ils produisent. On observera quelle est la force et le caractère de la jalousie chez les maris et chez les femmes; si l'adultère est puni à l'égard des uns ou des autres, et quelle est sa punition. En remarquant si la polygamie est établie, on examinera jusqu'où elle s'étend, quelles en paraissent être l'origine et la raison, quels en sont les effets, par rapport aux moeurs, à la population, à l'éducation des enfants, à la paix domestique, etc. 


\section{Éducation morale des enfants}

Il est certain qu' on ne peut s'attendre à trouver chez les sauvages des détails fort intéressants sur l'éducation morale des enfants. Cependant il y a plusieurs circonstances dignes de quelques remarques. Quel est le degré d'attachement, et la nature de l'intérêt que les parents portent à leurs enfants? Jusqu'où s'étendent à leur égard la surveillance et la sévérité? Est-ce le père ou la mère qui en prennent un soin plus particulier? Jusqu'à quel âge ce soin dure-t-il ? Est-il égal pour tous les enfants, ou témoignent-ils quelque préférence? Comment les enfants apprennent-ils la langue? Comment sont-ils initiés aux notions de morale que leurs parents peuvent avoir? Enfin, avec quelle rapidité se développent en eux les passions et l'intelligence?

\section{Société générale}

De la société domestique, passons à la société générale, celle qui est formée de l'agrégation des familles; elle se présente à nous sous quatre espèces différentes de rapports : les rapports politiques, civils religieux et économiques. Commençons par les rapports politiques, ceux qui servent de base à tous les autres.

\section{Rapports politiques}

\section{$1^{\circ}$ intérieurs}

Magistrats. Leur titre. Leur autorité

D'abord, quels sont les liens intérieurs de la société, et les fondements sur lesquels repose l'union de ses membres? Y a-t-il quelque gradation dans la formation de cette société, c'est-à-dire, se subdivise-t-elle, comme parmi nous, en plusieurs agrégations partielles, plus étroitement unies entre elles, telles que des bandes, ou des bourgades, ou des castes, et quels sont les rapports et les limites de ces associations particulières? Y a-t-il une distinction de rang? Sur quoi se fonde-t-elle? Est-elle attachée à la naissance? Par quelles prérogatives se manifeste-t-elle? De combien de degrés différents est-elle 
formée? Quel est le nombre des magistratures et celui des magistrats? Leurs fonctions sont-elles héréditaires ou électives, et quelles seraient les circonstances de cette élection? Quelle est la nature et l'étendue de leur autorité ? Quelle subordination règne entre eux? Quel esprit les dirige dans son exercice? Par quelles limites sont-ils arrêtés? Sont-ils à vie ou pour un temps? Sont-ils soumis à la [100] déposition, et comment s'exécute-t-elle? L'autorité suprême est-elle entre les mains d'un seul ou de plusieurs? Dans le premier cas est-elle absolue, arbitraire? Quelles circonstances l'accompagnent? Comment se transmet-elle? Sur quels objets se déploie-t-elle? Dans le second, comment les chefs sont-ils nommés? Comment s'accordent-ils entre eux? Leurs opérations sont-elles collectives; ou bien chacun a-t-il une administration séparée?

Quelles idées ces peuples ont-ils de l'autorité et de ses droits? Quels égards ont-ils pour leurs chefs? Quelle affection, quelle confiance, quelle soumission leur témoignent-ils? Quels hommages leur rendent-ils? De quelle pompe les entourent-ils?

\section{Ses effets}

Quels effets résultent de ces institutions? Jusqu'où s'étend I'union des membres de la société ? Quelle est sa solidité? Quelles sont les occasions, les circonstances, les effets des discordes civiles? Les révolutions sont-elles fréquentes, sanglantes? Les lois ont-elles besoin d'une sanction générale? Y a-t-il même des lois, ou si la volonté de ceux qui gouvernent en tient lieu? Comment les lois se conserventelles?

\section{$2^{\circ}$ extérieurs}

De quel œeil une peuplade considère-t-elle celles qui l'entourent et qui vivent sous une autre autorité ? Sont-elles naturellement en guerre, ou vivent-elles ordinairement en paix? 


\section{Guerre}

Si elles sont naturellement en guerre, d'où vient cette disposition mutuelle? Est-ce antipathie? Est-ce un effet de la vengeance et des souvenirs? Est-ce la rivalité ? Est-ce le désir des conquêtes?

\section{Causes}

Si les guerres sont accidentelles, quelles en sont les occasions les plus ordinaires?

\section{Circonstances}

Qui a le droit de déclarer la guerre? Cette déclaration est-elle précédée de quelque négociation, ou du moins de quelque formalité? Quelles sont ces formalités, ou quel est le caractère de ces négociations?

Les guerres sont-elles universelles, c'est-à-dire tout le peuple y prend-il part? Sont-elles sanglantes, longues? Suspendent-elles entre les peuples toute autre espèce de rapports et le sentiment des droits naturels?

\section{Art militaire}

Ces peuples ont-ils quelque espèce d'art militaire? Quelle subordination règne entre eux pendant la guerre et le combat? Marchent-ils dans quelque ordre? Observent-ils quelque concert pendant l'action? Cherchent-ils à se surprendre, ou s'attaquent-ils ouvertement? Les combats sont-ils longs, et la victoire longtemps disputée? Quelles causes décident ordinairement de la victoire? Quelles précautions [101] prennent-ils? Quelles sont les ressources qu'ils déploient dans la surprise ou après la défaite? 
Armes

Courage

On ne peut se plaindre que les voyageurs ordinaires nous laissent ignorer ni la nature des armes que les sauvages emploient, ni l'usage qu'ils en font: mais quelle est la nature et le degré de courage que ces peuples déploient? Est-ce celui de l'impétuosité, ou celui de la constance? Sont-ils furieux ou intrépides? Quelle idée se forment-ils du courage lui-même? Quel est le sentiment qu'ils ont de l'honneur, de la gloire et de l'indépendance?

Effets de la guerre

Jusqu'où s'étendent les effets de la guerre? Les femmes, les enfants, les habitations, sont-ils la proie du vainqueur, et quel sort éprouvent-ils de leur part? Les vainqueurs font-ils des prisonniers? Quels traitements leur font-ils éprouver? Quel est l'état et la condition des esclaves? Quels sentiments conservent-ils? N'y a-t-il d'autre esclavage que celui de la conquête?

Paix

Comment la guerre se termine-t-elle? Est-ce par la destruction entière, ou par l'expulsion des vaincus? Est-ce par quelque pacification? Comment la paix est-elle proposée, adoptée, garantie, et quelles en sont les conditions ordinaires?

\section{Alliance}

S'établit-il quelques alliances entre les peuplades voisines? Quelles en sont les occasions et les fins? Par quelles formalités sont-elles préparées? De quelles conditions sont-elles plus ordinairement formées? Quelle durée leur fixe-t-on? Sur quels fondements repose leur solidité? Quelles idées les peuples ont-ils du devoir qui les lie à leurs engagements, et quelle est leur bonne foi réciproque? Quels ta- 
lents développent-ils dans ces négociations, et quels sont les moyens qu'ils font valoir pour leurs succès?

\section{Étrangers. Hospitalité}

Quel accueil un peuple sauvage fait-il aux étrangers qui lui sont entièrement inconnus, comme les Européens? Quelle est la cause de cet accueil? C'est ici qu'il faut apporter bien des précautions, avant de porter un jugement. Il faut tâcher de s'assurer d'abord si l'accueil qu'on reçoit d'un peuple sauvage n'est pas l'effet des souvenirs que d'autres étrangers lui ont laissés; et alors il faudrait savoir encore quelle est la véritable conduite que ces étrangers ont tenue à son égard. Lors même qu'aucun souvenir ne pourrait influer sur la réception que l'on reçoit, il reste plusieurs manières d'expliquer une même réception. La crainte et la férocité peuvent également mettre les armes à la main du peuple que l'on visite ; la bonté, la confiance, la timidité ou la perfidie, peuvent également l'engager à faire aux étrangers un accueil favorable. Enfin, la férocité, la défiance, ou la douce vertu de l'hospitalité, sont elles-mêmes [102] des phénomènes moraux, dont il faut, autant qu'il est possible, chercher à pénétrer les causes.

\section{Propriétés}

Les institutions civiles ont pour objet de garantir aux membres de la société leurs propriétés et leur vie la plus précieuse de toutes.

Tel peuple sauvage a-t-il l'idée de la propriété ? S'il est pasteur ou chasseur, il n'a pas sans doute celle de la propriété territoriale, mais alors, n'a-t-il pas du moins celle de la propriété de ses instruments, et des objets qu'il a obtenus par ses efforts?

\section{Délits}


Les punitions des injures chez un peuple sauvage sont-elles abandonnées à la vengeance de l'individu qui les a reçues? S'il en est ainsi, quels sont les cas dans lesquels il s'attribue cette vengeance? Quelle vengeance exerce-t-il ? Le coupable en reconnait-il le droit? Où leur querelle ne paraît-elle que la lutte de la force contre la force? Si la punition des injures est réservée à quelque autorité, quelle est cette autorité ? De quels délits connait-elle? Comment les constate-t-elle? Quelle est la peine à laquelle elle les soumet, et quel est le principe d'après lequel elle paraît fixer ses arrêts?

\section{Rapports économiques}

\section{Territoire}

Le premier objet à remarquer, en considérant l'association sous ses rapports économiques, est la fécondité du territoire qu'un peuple habite, et l'abondance plus ou moins grande des ressources que son séjour lui présente.

\section{Première industrie}

Ensuite il faut observer comment il use de ce territoire et tire parti de ces ressources, s'il a commencé quelque culture, s'il en a quelque idée: s'il cueille du moins le fruit des arbres, s'il a apprivoisé quelques animaux, s'il se nourrit de leur chair ou de leur laitage, ou s'il ne cherche ses aliments que dans la chasse ou la pêche.

Il est assez difficile de s'expliquer comment un peuple chasseur est séparé, par une limite si prononcée, si durable, du peuple cultivateur? Comment se fait-il que l'idée ne vienne point à ce peuple d'essayer les aliments que présentent les fruits de la terre, et ensuite de chercher à les reproduire? Quel obstacle caché le retient dans la sphère de cette existence si laborieuse et si pénible, qui fait dépendre la satisfaction des premiers besoins, d'une course longue et périlleuse? Tel est le problème que nous proposons à la sagacité des voyageurs. Du moins est-il à propos d'essayer si on ne pourrait pas les engager à adopter un genre de vie plus heureux et plus commode, en leur 
en donnant quelques exemples, si on ne réussirait pas à leur enseigner l'art de la culture, à leur en faire sentir les bienfaits. Si on ne pouvait y parvenir, il faudrait alors chercher à pénétrer le motif d'une si singulière répugnance.

[103]

\section{Essais}

Cook et d'autres voyageurs ont cherché à transplanter nos animaux domestiques au milieu de diverses nations sauvages. Il est important de s'informer de ce qu'ils en ont fait; et dans les lieux où ces races ont été ou détruites ou dispersées, il faudrait savoir pour quelle raison on les a ainsi abandonnées. Nous insistons sur ces considération, parce que si l'on trouvait quelques moyens de faire passer les peuples sauvages à l'état de pasteurs ou d'agriculteurs, on ouvrirait sans doute devant eux la route la plus sûre qui puisse les conduire aux avantages de la civilisation.

\section{Vagabondage et séjour}

Au genre de vie que mène un peuple, se lie nécessairement le mode de son habitation. Il faudra donc nous apprendre si ces demeures sont fixes, ou si elles sont errantes: s'il change souvent de séjour; quels motifs le dirigent dans ses voyages.

\section{Industrie du second ordre}

Le seconde espèce d'industrie dont il faudra étudier le développement, est celle qui a pour objet les besoins de second ordre ou de commodité, comme la construction des huttes et la fabrication de vêtements. On s'attachera à décrire les méthodes que les sauvages suivent et les procédés qu'ils emploient: mais on cherchera aussi à connaître s'ils se perfectionnent dans ces travaux, ou pourquoi ils ne $s^{\prime} y$ perfectionnent pas: I'on fera quelque effort pour les engager à mieux s'y prendre, et on leur en indiquera les moyens. 


\section{Industrie auxiliaire}

La troisième espèce d'industrie est celle qui consiste à préparer les instruments de travail. Ici, les deux choses les plus importantes pour le sauvage, sont les métaux et le feu. S'ils connaissent les métaux, il faudra examiner comment ils les accommodent à leur usage. $S$ 'ils ne les connaissent pas, il faudra observer comment ils y suppléent ; il faudra essayer s'ils ne voudraient pas apprendre à s'en servir. Quant au feu, son ignorance atteste sans doute le degré le plus éloigné de la civilisation, et on n'a pas besoin de recommander aux voyageurs de leur procurer ce bienfait. Mais les peuplades mêmes qui font usage du feu, n'en connaissent pas tous les effets; et c'est ce qu'il serait assez curieux d'examiner.

La constructions des nacelles et pirogues dont se servent les sauvages, de leurs instruments de chasse et de pêche, la description des ruses et des procédés qu'ils emploient, de leur manière de naviguer, etc., sont des objets en général assez bien décrits par les voyageurs, parce qu'ils intéressent davantage la curiosité du vulgaire. Cependant on ne négligera point de compléter, s'il se peut, ces descriptions et ces peintures, et surtout de rapporter en Europe quelques modèles des divers instruments des sauvages. Il serait assez curieux de savoir si et comment un peuple sauvage marque les révolutions des années, des mois, des jours et des heures.

[104]

\section{Commerce}

Un peuple sauvage ne porte-t-il jamais dans ses rapports avec ses voisins quelque idée de commerce? Quelle est la facilité avec laquelle il se prête à opérer quelques échanges avec les étrangers qu'il visite? Sur quel principe fonde-t-il ces échanges? Quel est le prix et la valeur que les objets ont pour lui ? Est-il déterminé uniquement pour ses besoins? Attache-t-il quelque valeur à son superflu? Cherche-t-il à tirer quelques avantages de la demande qu'on lui en fait? Se dépouille-t-il facilement des objets dont il fait quelque usage? Porte-t-il quelque bonne foi dans ces transactions: ou, s'il est de mauvaise foi, $a-t-i l$ quelque sentiment de son tort? La plupart des voyageurs n'ont 
vu dans le commerce avec les peuples sauvages qu'un objet de spéculation, ou qu'un moyen de s'établir au milieu d'eux pour les dominer. Un voyageur philosophe porterait ses vues bien plus loin. Il verrait dans ce commerce un moyen de les conduire à la civilisation. En effet, ce n'est guère qu'avec notre secours qu'ils peuvent se civiliser; et le besoin seul peut les rapprocher de nous. Ainsi un premier échange facilitera de premières communication: ces communications serviront peutêtre à inspirer au sauvage quelques nouveaux désirs, qui le rappelleront encore auprès de nous. Toujours bien reçu, bien traité, témoin de notre bonheur, de notre richesse, en même temps que de notre supériorité, peut-être s'attachera-t-il à nous par la reconnaissance ou l'intérêt, formera-t-il avec nous quelque alliance, nous appellera-t-il au milieu de lui pour lui enseigner la route qui doit le conduire à l'état où nous sommes. Quelle joie! quelle conquête, s'il s'ouvrait pour nous quelque espérance d'exercer une douce et utile influence sur ces peuples abandonnés, et de renouveler dans les mers du Sud l'étonnante révolution du Paraguay.

\section{Arts d'amusement}

Il est assez singulier de voir quelquefois des peuples qui ont à peine le nécessaire, s'occuper de leurs plaisirs, et y attacher une grande importance. Cette observation excitera sans doute la curiosité des voyageurs. Ils étudieront aussi les plaisirs des sauvages, puisque c'est une partie de leur existence. Ils examineront leurs chants et leurs instruments de musique: ils chercheront à connaître s'ils ont quelque idée de poésie ils essaieront si leurs oreilles sont sensibles à une parfaite harmonie ils nous transmettront le détail de leur luxe, quelquefois si bizarre, et s'étudieront à pénétrer les idées qu'ils y attachent.

\section{Population}

Le dernier, et le plus important peut-être des objets que les rapports économiques présentent à l'observateur, sera la population. Il faudra reconnaître d'abord quelle est la proportion du nombre des individus qui habitent un territoire, à l'étendue de ce même territoire. [105] Il faudra s'informer ensuite si cette population croît ou décroît 
avec le temps, et à peu près selon quel rapport. Il faudra découvrir enfin quelles sont les véritables causes de ces accroissements ou de cette décadence.

\section{Rapports moraux et religieux}

Nous avons déjà parlé plus haut des notions qu'un peuple peut avoir de la religion et de la morale: il ne s'agit plus ici que des effets extérieurs qu'elles produisent à l'égard de la société.

\section{Vertus généreuses}

On observera si le sauvage connaît la pitié pour la faiblesse et le malheur, et s'il pratique cette bienfaisance qui vient à son secours: jusqu'où s'étendent en lui ces sentiments: s'il sait adjoindre cette générosité qui relève le bienfait par le sacrifice. Répond-il aussi au bienfait par la reconnaissance? Quelle est la durée, quels sont les signes extérieurs de cette reconnaissance? Jusqu'à quel point se croitil obligé envers son bienfaiteur, et quelle est I'horreur qu'il témoigne pour l'ingratitude?

\section{Vertus fortes}

On observera s'il est capable de supporter quelques offenses, et jusqu' où il porte la patience: quel est le caractère de sa vengeance; si rien ne peut la fléchir ; si elle va au-delà de l'offense même; s'il y attache quelque idée de justice comme à une espèce de talion; s'il s'élève quelquefois jusqu'à sentir le mérite du pardon; et s'il sait obtenir quelque empire sur les passions de son cœur.

\section{Affections. Amitiés}

Il observera à quel point le cour du sauvage peut être sensible aux affections qui unissent les hommes entre eux, s'il se livre à ce noble sentiment de l'amitié dont des nations semblables nous ont souvent 
présenté de touchants exemples. Alors il étudiera le caractère d'une telle amitié, son origine, ses effets, ses signes, sa durée. Heureux s'il rapporte aux philosophes quelques nouvelles preuves de l'existence de ce sublime instinct qui porte I'homme vers son semblable, et de son intime inhérence à notre nature!

\section{Amour de la patrie}

Le sentiment de l'amitié doit être considéré peut-être comme I'origine de toutes les affections sociales; car la bienveillance s'arrête sur ce qui nous entoure avant de s'étendre au-delà ; elle se fixe sur l'individu, avant d'embrasser la société. Qu'est-ce dans le cœur du sauvage que l'amour de la patrie? Est-ce un sentiment d'affection pour tous ceux qui vivent avec lui dans une société commune? Est-ce l'attachement au sol qu'il occupe, à la vie qu'il mène, aux habitudes par lesquelles il est dominé? Est-ce un retour sur son propre intérêt? Quelle est la force de ce patriotisme, et par quels signes se manifeste-t-il au-dehors? Saurait-il se dévouer pour l'avantage de sa propre société ? Quel est le besoin qu'il a de la liberté, la honte qu'il attache [106] à l'esclavage, et la haine qu'il porte au pouvoir arbitraire et despotique? Connait-il aussi l'ambition du pouvoir et sous quelle forme se présente-t-elle à ses yeux?

\section{Cérémonies religieuses}

Le sauvage regarde-t-il le culte extérieur et les cérémonies comme nécessairement liés à l'idée qu'il a d'un Être suprême et de ses devoirs envers lui ? Est-ce dans le dessein de l'honorer, de le fléchir, de lui rendre grâces, qu'il décerne un culte à l'Être suprême, ou croit-il contribuer par là aux jouissances de cet Être, en l'assimilant en quelque sorte à lui-même?

\section{Prêtres}

Quel est le nombre de ses prêtres? De quelle manière sont-ils choisis? Quelle considération, quels privilèges, quelle autorité leur accorde-t-il ? Leur suppose-t-il quelque pouvoir sur la nature, quelque 
faculté pour pénétrer l'avenir, ou découvrir l'inconnu? Ces prêtres ont-ils quelque degré d'instruction particulière? Paraissent-ils de bonne foi ? Ont-ils en général plus de moralité que le reste du peuple? Comment vivent-ils entre eux? Pratiquent-ils la médecine, et d'après quelles idées? L'influence qu'ils exercent paraît-elle salutaire? Se montrent-ils disposés à seconder quelques vues d'amélioration et de perfectionnement ; ou bien se montrent-ils intéressés à maintenir leur nation dans l'ignorance et la barbarie?

\section{Temples. Idoles}

Ce ne sera pas assez d'avoir décrit, comme on le fait ordinairement, la forme des temples, et la figure des idoles qui servent d'objet à la vénération d'un peuple; il faudra nous faire connaître quelles sont les idées que ce peuple attache à ces idoles, à ces temples si toutefois il en attache quelqu'une. Il faudra s'assurer si c'est là le dernier objet de son culte, ou s'il ne considère ces objets que comme des signes.

Il en sera de même des diverses cérémonies, qui toutes bizarres qu'elles paraissent, ou plutôt par là même qu'elles sont très bizarres, doivent avoir eu quelque raison particulière.

\section{Actes religieux}

Un peuple sauvage a-t-il des jours de fête qui soient fixés? Quelle est l'occasion et l'esprit de ces fêtes? La naissance des enfants, le mariage, la mort, la sépulture, l'élection des magistrats, la guerre, la paix, les calamités générales sont-elles pour lui le sujet de quelques actes religieux? Quels sont ces actes? Y a-t-il des exemples d'actes religieux, qui soient répétés chaque jour? Prononcent-ils des prières en commun, et quelles sont ces prières? Ont-ils quelque formule de malédiction pour leurs ennemis?

\section{Tombeaux}

Quel est le culte qu'un peuple a pour les morts, et le respect qu'il porte aux tombeaux? 


\section{Traditions}

Le dernier objet de la curiosité des voyageurs, et le plus difficile sans doute à obtenir, sera de pénétrer les traditions des peuples sauvages. [107] Ils les interrogeront sur leur origine, sur les transmigrations qu'ils ont éprouvées, sur les invasions auxquelles ils ont été exposés, sur les visites qu'ils ont reçues, sur les évènement importants qui se sont passés au milieu d'eux, sur les progrès qu'ils ont pu faire sous les rapports de l'industrie ou de la force politique, sur l'institution des coutumes en usage parmi eux. Peut-être n'en tireront-ils que des récits bien vagues: mais un petit nombre de faits peuvent jeter une précieuse lumière sur la mystérieuse histoire de ces nations.

Nous ne terminerons point sans recommander aux voyageurs de nous ramener, s'ils peuvent, des sauvages des deux sexes, partie dans l'âge de l'adolescence, partie dans l'enfance, et de les préparer, par les meilleurs traitements, à l'adoption qui leur sera destinée.

Il serait à désirer surtout qu'ils pussent engager une famille entière à les suivre. Alors les individus qui les composent, moins contraints dans leurs habitudes, moins attristés par les privations, conserveraient davantage leur caractère naturel. Ils consentiraient plus facilement à se fixer au milieu de nous; et les rapports qui existeraient entre eux, rendraient pour nous le spectacle de leur vie à la fois plus curieux et plus utile. Nous posséderions en petit l'image de cette société, à laquelle ils auraient été enlevés. Ainsi le naturaliste ne se contente pas de rapporter une branche, une fleur bientôt desséchée: il cherche à transplanter la plante, l'arbre tout entier, pour lui rendre sur notre sol une seconde vie. 


\section{Conclusion}

Nous n'ignorons pas que l'ensemble des problèmes que nous soumettons à la sagacité des voyageurs, demande un travail immense, soit par le nombre et l'importance même des questions, soit par le détail et l'assiduité des observations qu'exige chacune d'entre elles. Nous n'ignorons pas que ce travail est entouré de difficultés de toute espèce et qu'on doit s'attendre à éprouver de grands obstacles dans les premiers rapports qu' on voudra établir avec les sauvages. Car ces peuples ne peuvent pénétrer les intentions véritables de ceux qui les approchent, ils ne peuvent distinguer facilement leurs amis de leurs ennemis, et ceux qui leur apportent des secours, de ceux qui viennent envahir leur territoire. Mais il n'est rien que nous n'ayons droit d'espérer de la patience, de la persévérance, du courage héroïque des voyageurs qui reçoivent aujourd'hui nos adieux; nous en avons la garantie dans leur caractère personnel, dans les vues qui les animent, dans les preuves éclatantes qu'ils en ont déjà données. Eh! que n'ontils pas fait déjà pour les sciences, et quelle noble carrière n'ont-ils pas déjà courue! Il était digne d'eux d'en reculer encore le terme, et [108] d'aller achever un si bel ouvrage! Hommes estimables, en vous saluant ici à la veille d'un prochain départ, en vous voyant vous arracher à votre patrie, à votre famille, à vos amis, et vous élancer hors des limites du monde civilisé ; en fixant l'image des fatigues, des privations, des dangers qui vous attendent, et de ce long exil auquel vous vous êtes volontairement condamnés, nos âmes ne peuvent se défendre d'une émotion profonde, et le mouvement de la sensibilité s'unit en nous au respect que nous devons à de si nobles travaux. Mais notre pensée fixe d'avance le terme auquel ils doivent atteindre; et, en s'arrêtant à cette perspective, tous nos sentiments se confondent en celui de l'admiration et de l'enthousiasme. Illustres messagers de la philosophie, pacifiques héros, les conquêtes que vous allez ajouter au domaine des sciences, ont plus d'éclat et de prix à nos yeux que les victoires achetées par le sang des hommes! Tous les cœurs généreux, tous les amis de l'humanité s'associent à votre sublime mission; il y a dans cette enceinte plus d'un cœur qui vous porte envie, qui gémit en 
secret que des devoirs inflexibles le retiennent sur ces rivages, qui mettrait sa gloire à vous suivre, à vous imiter. Nos voux vous accompagneront du moins au travers de l'océan, ou dans le sein des déserts: notre pensée vous visitera souvent, lorsque sous l'équateur ou près du pôle, vous recueillerez en silence de précieux trésors pour les lumières. Nous nous dirons « $A$ ce jour, à cette heure, ils abordent peutêtre sur une terre inconnue, ils pénètrent peut-être au sein d'un peuple nouveau, peut-être ils se reposent à l'ombre d'antiques forêts de leurs longues souffrances ; peut-être ils commencent à entrer en rapport avec une nation barbare, à effacer ses farouches préventions, à lui inspirer la curiosité de connaître nos mœurs et le désir de les imiter, et peut-être ils jettent le fondement d'une nouvelle Europe ». Eh! qui dira en effet tous les résultats possibles ou probables qui peuvent naître un jour de ces belles entreprises? Je ne parle pas seulement ici de nos cabinets enrichis, de nos cartes rectifiées ou étendues, de nos connaissances sur l'histoire physique et morale du monde multipliées et agrandies, du nom Français porté sur des rives inconnues! Que d'autres perspectives séduisantes s'offrent encore à l'imagination étonnée! Le commerce étendu par de nouvelles relations: la marine perfectionnée par une plus grande expérience; les voyages facilités par les découvertes; notre grandeur politique accrue par de nouvelles colonies ou de nouvelles alliances! Que savons-nous? Peut-être des nations entières civilisées, recevant de la civilisation le pouvoir de se multiplier, en s'associant à nous par les liens d'une vaste confédération: peut-être de plus vastes et plus utiles carrières ouvertes à [109] l'ambition, au génie et à l'industrie humaine; ces peuples de l'Europe qui se disputent chaque jour au prix de leur sang quelque étroit territoire, s'étendant à loisir dans de plus belles contrées un monde nouveau se formant peut-être aux extrémités de la terre; le globe entier couvert d'habitants plus heureux et plus sages, plus également partagés, plus étroitement unis, la société s'élevant à de plus rapides progrès par une plus grande émulation, et atteignant peut-être par ces révolutions inattendues, ce perfectionnement qu'invoquent nos vœux, mais auquel contribuent si peu et nos lumières, et nos méthodes, et nos livres !... Vaines chimères peut-être; mais chimères auxquelles nos longs malheurs, nos tristes dissensions, et le spectacle de notre corruption, donnent cependant tant de charmes !... Du moins est-il certain 
que ces hardies entreprises, formées vers les parties les plus reculées de l'univers, préparent pour la postérité un nouvel avenir, et qu'il ne tiendra qu'à la sagesse de nos descendants de cueillir des fruits abondants dans cette carrière que vous allez ouvrir. Voyez combien les découvertes de Colomb changèrent la face de la société, et quelles étonnantes destinées porta ce fragile navire auquel il s'était confié! Il est vrai, cette grande révolution n'a pas toute été à notre avantage, encore moins à celui des peuples auxquels il nous a ouvert un accès. Mais Colomb ne jeta dans le Nouveau Monde que d'avides conquérants; et vous ne vous avancez vers les peuples du Sud qu'en pacificateurs et en amis. Les farouches aventuriers de l'Espagne ne portèrent devant eux que la destruction, et vous ne répandrez que des bienfaits. Ils ne servaient que les passions de quelques hommes, et vous n'aspirez qu'au bonheur de tous, qu'à la gloire d'être utiles! Elle vous attend, elle vous environne déjà cette gloire, la plus douce, la plus vraie, ou plutôt la seule vraie, vous en connaîtrez tout l'éclat, à ce jour de triomphe et de joie, auquel, rendus à votre patrie, reçus au milieu de nos transports, vous arriverez dans nos murs, chargés des plus précieuses dépouilles, et porteurs d'heureuses nouvelles de nos frères dispersés aux derniers confins de l'univers. 
[111]

Aux origines de l'anthropologie française. (1994)

Édition revue et corrigée par les auteurs, 1993.

\section{Texte 4}

\section{Observations sur l'anthropologie ou l'histoire naturelle... (1800)}

\section{Par François Péron}

\section{Retour à la table des matières}

Le titre complet du mémoire est : Observations sur l'anthropologie ou I'histoire naturelle, la nécessité de s'occuper de l'avancement de cette science, et l'importance de l'admission sur la flotte du capitaine Baudin d'un ou de plusieurs naturalistes, spécialement chargés des recherches à faire sur ce sujet.

L'original de ce mémoire est conservé à la bibliothèque du Muséum national d'Histoire naturelle sous la cote : Y 1. 4186. Georges Hervé le reproduisit dans un article intitulé : «Les premières armes de Péron 》, Revue anthropologique, vol. XXIII, 1913, pp. 1-16.

François Péron rédigea ce mémoire à la demande de Georges Cuvier, de qui il était élève, et le lut ou le fit lire lors d'une séance solennelle de l'Institut de France. Cette intervention, contemporaine de la démission d'un des naturalistes de l'expédition, lui permit d'être 
recruté comme zoologiste et anthropologiste, - bien que, dans son mémoire, Péron se soit surtout attaché à défendre des considérations médicales et à revendiquer une position de médecin. Grâce à l'appui de ce même Cuvier et de Lacépède, Péron put donc embarquer au Havre le 19 octobre 1800. «Arrivé le dernier, écrit M. Girard dans F. Péron, naturaliste, voyageur aux terres australes (Paris, J. B. Baillère et fils, 1857, p. 2 1) et sans être attendu, il trouva toutes les places occupées et, de là, mille privations, mille ennuis. »

Ce furent surtout les conditions précipitées de ce recrutement, ajoutées à la jeunesse (il avait vingt-cinq ans) et au caractère entier du personnage, qui ont été la cause de ces ennuis dont parle Girard et, notamment, des tensions qui se sont manifestées [112[ entre Baudin et lui. Animé d'un zèle intempestif qui lui fit risquer sa vie et celle de ses compagnons plusieurs fois au cours du voyage, François Péron se heurta très tôt et à nombre de reprises au réalisme et à l'autoritarisme de Baudin.

Il est en tout cas surprenant d'observer que l'expédition à laquelle Péron participa et dont il se voulut être «le médecin et l'anthropologiste», revint pour ainsi dire exsangue à Lorient le 25 mars 1804. Baudin, victime d'une maladie pulmonaire, mourut sur le chemin du retour. Sur les vingt-quatre savants, artistes et techniciens que comptait l'expédition et qui étaient répartis sur les deux corvettes la composant, Le Géographe et Le Naturaliste, seulement six revinrent indemnes (Péron devait cependant mourir moins de six ans plus tard, à trente-cinq ans, des suites d'une maladie contractée pendant le voyage). Dès l'aller, dix avaient été débarqués, malades, à l'Ile de France le 25 avril 1801. Huit périrent en cours de route. La sécheresse de ces chiffres dénonce en vérité les conditions d'hygiène déplorables dans lesquelles vécurent les membres de l'expédition. Cruelle ironie du sort pour ceux qui, tel Péron, entendaient faire de l'hygiène une discipline visant à «perfectionner la nature humaine»! 140 Tout aussi cruelle

140 Cf. J. VERNES, Les Grands navigateurs du XVIIIe siècle, Paris, Ramsay, 1977 (édition originale: 1879); J.-P. FAIVRE, L'Expansion française dans le Pacifique, 1800-1804, Paris, Nouvelles éditions latines, 1953 ; J. JAMIN, « Faibles sauvages... Corps indigènes, corps indigents. Le désenchantement de François Péron », in J. HAINARD \& R. KAEHR, Le Corps enjeu, Neuchâtel, Musée d'eth- 
cette démonstration en quelque sorte par l'absurde des thèses de Péron, qui soutenait que la «perfection morale » (les civilisés) est en raison inverse de la «perfection physique» (les sauvages). L'expédition Baudin devait en apporter la funeste preuve...

Une grande expédition se prépare... Des savants de toute espèce vont aller au-delà du tropique du Capricorne, dans des climats presque entièrement inconnus encore, cueillir une ample moisson d'observations utiles. Ce groupe respectable de savants, toutes les sciences ont concouru à le former... La médecine seule, par une exclusion non moins injurieuse pour elle que funeste à la société, la médecine seule n'est point appelée au partage des dangers et des succès d'une aussi noble entreprise... Eh! quelle autre cependant, pourrait en retirer des avantages plus grands, plus immédiatement utiles à l'humanité ?

Sans doute il est beau d'aller cueillir à grands frais la mousse inerte qui végète sous les glaces éternelles des pôles: sans doute il est beau d'aller poursuivre jusqu'au fond des déserts brûlants du Sahara ces reptiles hideux que la nature semblait y avoir exilés pour nous mettre à l'abri de leur fureur: mais ayons le courage de le dire, serait-il moins beau, serait-il moins utile à la société, d'associer aux naturalistes, chargés de ces recherches importantes, quelques jeunes médecins spécialement [113] destinés à l'étude de l'homme, à recueillir tout ce que les peuples divers peuvent présenter d'intéressant dans leurs rapports physiques et moraux avec le climat qu'ils habitent, leurs mours, leurs habitudes, leurs maladies tant internes qu'externes, et les moyens de les prévenir ou de les soulager...?

Nous avons peu de choses à désirer sur la médecine des régions situées entre l'un et l'autre tropique: une foule d'excellents auteurs, dont aucun cependant n'est français, nous ont donné des recherches

nographie, 1983, pp. 45-76; T. GUICHETEAU \& J.-P. KERNEIS, «Étude à travers les chrononavigrammes des responsabilités médicales de Baudin en Australie en 1801-1803 », in Les Français et I'Australie, Paris, Université Paris XNanterre, 1989, pp. 141-156 
précieuses sur les maladies équatoriales dans l'un et l'autre hémisphères : il suffit de citer Cleyerus de Medicina Sinorum; Bontius de Medicina Indorum; Prosper Alpinus de Medicina AEgyptiorum; Pison (Guillem.) de Medicina Brasiliensium: Margravius de Medicina Chiliensium, etc.

Il n'en est pas de même des pays plus reculés vers les pôles: nous n'avons encore aucun ouvrage sur la médecine de ces contrées rigoureuses... Ne serait-il pas glorieux pour la nation française de devancer les autres peuples dans cette nouvelle et importante carrière qui ne saurait manquer de fournir les résultats les plus grands et les plus utiles à la fois... ? La flotte doit, dit-on, séjourner longtemps à la Nouvelle-Hollande, à la terre de Diémen, à la Nouvelle-Zélande : quel théâtre plus propice à faire des observations neuves et intéressantes, que ces contrées immenses sur lesquelles les voyageurs et les historiens ont dit si peu de chose, et les médecins rien du tout encore...?

Déterminer la nature physique du climat, rechercher et préciser son influence sur la constitution organique des peuples qui I'habitent ainsi que sur le développement de leurs facultés morales et intellectuelles, étudier leurs passions dominantes, en rechercher la cause, décrire leurs occupations, leurs travaux, leurs exercices, détailler enfin tout ce qui peut avoir rapport à leur hygiène: telle devrait être la première partie de ce travail intéressant.

La seconde ne présenterait pas moins d'utilité dans ses résultats: ce serait d'observer avec soin tout ce qui concerne la médecine proprement dite, d'étudier attentivement toutes les maladies internes, soit générales, soit sporadiques, et surtout celles endémiques; ce serait d'en décrire scrupuleusement tous les symptômes, indépendamment de toute théorie, de toute hypothèse: d'en noter la marche et la terminaison: de s'appliquer surtout à en distinguer les phénomènes particuliers dépendant soit du climat, soit du tempérament physique ou moral, des habitudes, du mode de vivre et de la nature des aliments de ces peuples; il faudrait tâcher en s'aidant des lumières de la physique, de la chimie, de la géographie, de l'histoire naturelle, il faudrait, disje, tâcher de remonter aux causes de ces endémies et aux moyens de les prévenir ; il faudrait enfin, et ce dernier objet est de la plus haute importance, s'occuper de la connaissance de ces remèdes précieux que 
l'instinct et l'expérience firent connaître à ces hommes sauvages, à qui déjà nous devons tant de médicaments utiles et notamment le quinquina, la serpentaire, le sassafras, etc. Eh! n'en doutons pas, ces mêmes sauvages pourraient encore nous indiquer des substances non moins précieuses que le quinquina. Plus près que nous de la nature, forcés [114] par le besoin de perfectionner tous leurs sens, est-il étonnant qu'ils aient été mieux servis par l'instinct que nous ne l'avons été nous-mêmes par nos méthodes, quelque savantes qu'elles puissent être...? «Quand je considère, s'écrie Pison, quelles cures merveilleuses opèrent tous les jours ces hommes sauvages (les Brésiliens), avec les plantes les plus communes et les plus viles en apparence, je suis tenté de m'écrier avec Pline: Quel démon dans sa fureur enseigna donc aux hommes l'art perfide de préparer ces poisons pharmaceutiques qui ne sont propres qu'à servir l'avare cupidité du médecin aux dépens du malade... ! »

Des recherches sur les maladies chirurgicales ne seraient pas non plus sans intérêt et sans utilité : certes, il doit être beau, il doit être avantageux de voir comment la nature et l'instinct ont pu suppléer à l'art que sans doute ils ignorent.

En général, l'une et l'autre branches de la médecine curative ne sauraient manquer de retirer le plus grand profit de la comparaison des maladies polaires avec celles équatoriales. N'est-on pas effrayé de la marche violente et rapide de ces dernières? Tantôt ce sont des tétanos, des trismus qui saisissent instantanément, et tuent en quelques heures I'homme le plus robuste ${ }^{141}$. Tantôt ce sont des fièvres tellement aiguës, que le jour même de l'invasion est celui de la mort du malade ${ }^{142}$. Toutes les maladies inflammatoires présentent le même caractère d'exaspération, et sont accompagnées des symptômes les plus effrayants 143. L'inflammation du rectum, endémique au Brésil, se termine en peu de jours par la gangrène et la Mort ${ }^{144}$. Là 145 règnent

141 PISON, p. 31 ; BONTIUS, p. 23.

142 PROSPER-ALPIN, p. 24.

143 VOLNEY, t. 1er, p. 217 et 228 ; PROSPER-ALPIN, p. 23, 28 ; PISON, p. 15, 32 ; BONTIUS, p. 24.

144 PISON, p. 42. 
des endémies d'amauroses, d'héméralopie, de nyctalopie: ici ${ }^{146}$ ce sont des paralysies de la moitié du corps à la fois. Ailleurs 147 ce sont des ophtalmies si communes et si graves, que la quantité des aveugles est prodigieuse. Les maladies chirurgicales ne sont pas accompagnées d'accidents moins terribles; les plaies surtout, même les plus légères, sont très sujettes à devenir gangreneuses, à se couvrir de vers, ce qui le plus ordinairement entraîne la perte du malade 148.

Sans doute les maladies des climats plus rapprochés des pôles doivent présenter un ensemble de phénomènes bien différents: sans doute leur marche n'est pas aussi rapide, sans doute la température différente du climat, la différence de la constitution physique des peuples qui vivent dans ces contrées rigoureuses, la différence de leurs aliments, de leurs institutions sociales, etc., doivent produire une foule de modifications qu'il serait infiniment utile de recueillir pour l'avancement de la [115] médecine. Par quelle inconcevable contradiction de l'esprit humain se fait-il donc que tandis qu'il n'est presque aucune contrée dont nous ne connaissions les nombreuses productions végétales, nous ignorions jusqu'au nom même des maladies répandues sur plus des trois quarts de la surface du globe?

Indépendamment de tous ces grands objets de recherches, combien n'en est-il pas d'autres non moins importants pour l'avancement de l'histoire naturelle de l'homme? Quel avantages ne pourrait-on pas retirer de l'étude de ces nations sauvages que nous connaissons sous la dénomination, bien souvent injuste, de Barbares que leur donnèrent les aventuriers spoliateurs, ou les marchands avides qui, presque seuls encore, nous les ont fait connaître?

Ne serait-il pas utile par exemple de chercher à déterminer à quelle cause ou plutôt à quel concours de circonstances physiques et morales on peut attribuer la santé presque inaltérable et la longévité presque miraculeuse dont jouissent les peuples sauvages ou plutôt les na-

145 Le Brésil, PISON, p. 30.

146 L'Inde, BONTIUS, p. 22.

147 L'Égypte, PROSPER-ALPIN ; VOLNEY, les Mémoires de l'Institut national du Caire.

148 PISON, p. 43. 
tions qui s'éloignent le plus de la civilisation européenne... ? Le fait est incontestable; le témoignage des voyageurs et des médecins les plus distingués est unanime sur ce point... Ces avantages, bien précieux sans doute, ils ne les doivent pas uniquement à la nature physique du climat, à sa température: ils ne les doivent pas uniquement non plus à leur conformation organique, aux caractères particuliers de leurs aliments, ni même à leur genre de vie habituelle: en effet, nous les trouvons, ces avantages, dans cette race d'hommes hideux et dégénérés qui végètent au milieu des glaces de la Laponie, se nourrissant en grande partie de poisson desséché et passant des mois entiers engourdis dans leurs huttes enfumées et ensevelies sous la neige et les frimas: nous les trouvons dans I'habitant brûlé du désert, qui toujours sous la tente, ne vivant que du lait de ses chameaux, mène depuis des siècles une vie continuellement errante et vagabonde; nous les trouvons dans cette belle race d'hommes sauvages, qui libres également de peines morales et d'infirmités physiques, coulent si paisiblement leurs jours innocents au milieu des délicieuses et immenses forêts du Brésil, ne se nourrissant que des fruits savoureux que la nature libérale prodigue a cet heureux climat... L'apathique habitant des Antilles, et cette race immonde qui peuple la pointe méridionale de l'Afrique, et qui se nourrit presque uniquement de poissons pourris, nous présentent encore les mêmes phénomènes. Nous les retrouvons dans l'habitant malheureux de ces contrées affreuses où le sauvage Oby roule à peine ses flots glacés; et toutes ces nations, essentiellement différentes par la nature du climat, par leur conformation physique, leur genre de vie, leurs aliments, etc.; toutes ces nations, dis-je, ne se rapprochent qu'en un seul point, le défaut de civilisation... Quelle source féconde de méditations pour le médecin philosophe...!

En effet, quelle cohorte effroyable de maladies de toute espèce qui n'ont d'autre source que les progrès même de notre civilisation...! Délivrez l'humanité de toutes celles qui ne dépendent que de l'entassement des hommes dans les cités trop populeuses; de toutes celles produites par les différents travaux dans les arts; de toutes [116] celles que traîne à sa suite l'étude des sciences elles-mêmes; de toutes celles déterminées par le bouleversement des empires et la fureur des nations; de toutes celles enfin qui ne proviennent que de l'exaspération de nos passions; et le nombre de ces dernières seules est im- 
mense 149 ; délivrez, dis-je, l'humanité de toutes ces maladies nécessairement inconnues à des peuples moins civilisés, de combien alors la somme de leurs infirmités est moindre que la nôtre !...

A Dieu ne plaise que je prétende ici déclamer contre la civilisation: mais cette courte digression m'a semblé nécessaire pour prouver l'étroite connexion de la médecine, même pratique, avec la philosophie morale; elle m'a paru indispensable pour prouver qu'il était impossible de parvenir jamais à bien faire I'histoire naturelle de I'homme, tant qu'on se bornerait à étudier l'homme dégénéré et avili de la société. N'est-ce pas parmi ces peuples qui s'éloignent le plus de notre civilisation, et qui, conséquemment, sont encore plus près de la nature, n'estce pas chez eux qu'il faut aller puiser les éléments de cette histoire? N'est-ce pas dans le sauvage seul qu' on peut retrouver encore les traces de la robuste majesté de l'homme naturel : tout ce que les voyageurs et les médecins nous racontent du développement de la force physique des peuples non civilisés, et des actions qui en dérivent, tient vraiment du prodige... La perfection de leurs organes des sens est admirable... Par quels moyens parviennent-ils à ce haut degré de perfection physique... ? Ne pourrait-on pas faire l'application heureuse de quelques-uns de ces moyens à nos établissements sociaux...?

149 Les nombreuses et importantes observations recueillies avec tant de soin, à I'hospice de Clinique interne de l'École de médecine, par les, citoyens Corvisart et Leroux, sur les maladies organiques, ces observations, dis-je, prouvent évidement que la plupart de ces maladies et spécialement celles de l'estomac et du cœur, qui malheureusement sont d'une fréquence que, sans le zèle de ces praticiens distingués, on n'eût pas même osé soupçonner, reconnaissent principalement pour causes des chagrins profonds, des passions trop vives.

Les observations non moins exactes du citoyen Pinel, autre professeur illustre de l'École, prouvent également que la plus grande partie des fièvres adynamiques et ataxiques que, depuis plusieurs années, on observe en grand nombre à l'hospice de la Salpêtrière, n'ont pas une cause différente. Toutes les maladies nerveuses en ont-elles d'autres elles-mêmes? Inconnues des anciens, absolument ignorées des peuples sauvages, bien rares encore parmi les vigoureux habitants des campagnes, ces maladies, depuis quatre-vingts ans seulement, sont devenues presque générales dans les grandes cités... 
De telles recherches, qui ne peuvent être faites avec fruit que par des médecins, mais des médecins philosophes, deviendraient sans doute utiles; du moins devrait-on le tenter, et rien encore n'est fait à cet égard.

Ce que nous savons de l'insensibilité physique et morale de ces peuples sauvages 150 , ne serait-il pas bien digne aussi de fixer l'attention des Philosophes et [117] des naturalistes? Sans doute des recherches à cet égard pourraient conduire à une foule de résultats du plus grand intérêt pour I'histoire physique et morale de l'homme. Je ne dois entrer ici dans aucun détail sur les idées entièrement neuves que peut présenter un tel sujet: je me contenterai donc d'établir deux doutes bien importants, et à la solution desquels l'étude de l'homme sauvage peut seule fournir des données suffisantes.

150 Il serait peut-être assez difficile de déterminer lequel nous étonne le plus ou l'insensibilité physique du sauvage qui, du sein même des flammes qui le dévorent tout vivant, insulte à la rage impuissante de ses bourreaux, et meurt sans pousser un seul cri de douleur, ou bien l'insensibilité morale de ses bourreaux eux-mêmes, qui pendant ce sacrifice épouvantable chantent joyeusement et dansent autour du bûcher, attendant avec impatience le repas de cannibales dans lequel ils vont dévorer les membres encore palpitants de leurs malheureuses victimes... L'une et l'autre répugnent tellement à notre manière de sentir et de juger que nous avons peine à en concevoir la possibilité. Certains philosophes ont appelé l'une grandeur d'âme, courage héroïque, etc., mais tous les hommes civilisés traitent l'autre de férocité... Peut-être, en s'aidant des principes de la physiologie, verrait-on que ce n'est rien de tout cela; mais que l'impassibilité physique de la victime, et la farouche indifférence de l'assassin dépendent en grande partie de l'organisation physique de l'un et l'autre, et en sont une conséquence effroyable sans doute, mais nécessaire. Peut-être pourrait-on porter le même jugement de cette stoïque impassibilité des Spartiates: en analysant les institutions vraiment singulières du législateur de Lacédémone, il est aisé de sentir que Lycurgue avait eu pour but unique le développement de la force physique, aux dépens même de toutes les facultés morales et intellectuelles; et ces actions d'insensibilité physique et morale, sans doute trop vantées, ne me semblent prouver autre chose, sinon que le législateur avait atteint son but, un très grand degré de perfection physique. 
$1^{\circ}$ La perfection morale ne doit-elle pas être en raison inverse de la perfection physique?

$2^{\circ}$ Cette perfection physique n'exclut-elle pas non seulement la sensibilité morale, mais encore la sensibilité physique ellemême 151 ?

\section{Aux citoyens professeurs de l'École de médecine}

Vous pourrez voir, citoyens professeurs, dans les observations qui précèdent, l'exposition du plan de travail que je m'étais proposé lorsque je demandai à faire partie de l'expédition du capitaine Baudin : mon entreprise, sans doute, était immense, je le sentais bien: mais encouragé par l'approbation que plusieurs d'entre vous, à qui je l'avais communiquée, lui avaient donnée; dirigé même d'après les plans particuliers que plusieurs aussi d'entre vous se proposaient de me tracer ; profitant d'ailleurs des recherches et des observations de tous les autres savants de l'expédition: soutenu par un zèle ardent, une activité sans bornes, l'amour de l'étude, de l'observation et de la vérité: animé du glorieux désir de répondre à la confiance dont j'eusse été honoré : que de motifs puissants d'espérer que mon voyage n'eût pas été inutile pour mon pays, pour les sciences en général, et surtout pour celle de l'homme en particulier! [118]

Les démarches que j'ai faites ont été trop tardives: malgré les recommandations les plus pressantes et les plus flatteuses de la plupart d'entre vous et de plusieurs autres savants à qui

151 Notre savant professeur, le citoyen Hallé, a déjà émis dans son cours d'hygiène, une foule d'idées non moins nouvelles que philosophiques sur cet important objet. 
j'avais fait part des vues que je me proposais dans ce voyage: malgré le désir même que m'a témoigné le citoyen de Jussieu de favoriser mon utile entreprise, je n'ai pu rien obtenir, le nombre des places déterminées pour cette expédition s'étant trouvé rempli.

Une démarche auprès du Gouvernement ou de l'Institut national ne pourrait-elle pas, citoyens professeurs, vous faire obtenir l'autorisation nécessaire pour faire recevoir à bord de la floue un ou plusieurs jeunes étudiants en médecine, spécialement chargés sous le titre d'anthropologistes, de recueillir, d'après et conformément aux instructions que vous leur auriez tracées, toutes les observations propres à hâter le perfectionnement des diverses branches de la médecine? Dans le cas où vous jugeriez, citoyens professeurs, qu'un tel voyage pût en effet contribuer à l'avancement de notre art et à l'avantage de la société, il serait instant de faire les démarches nécessaires; le départ de l'expédition étant très prochain.

Dans le cas enfin où, suffisamment autorisés à faire partir quelqu'un, vous ne me jugeriez pas indigne d'être honoré de votre choix, j'ose vous assurer du zèle le plus soutenu, de la constance la plus infatigable pour répondre à cette confiance; et j'ose vous promettre que je ne négligerai rien pour que l'École ait un jour à s'applaudir d'une telle démarche. 
[119]

Aux origines de l'anthropologie française. (1994)

Édition revue et corrigée par les auteurs, 1993.

\section{Texte 5}

\section{Le Chinois Tchong-A-Sam (1800)}

\section{Par Louis-François Jauffret et Leblond}

\section{Retour à la table des matières}

Ce texte a été édité et publié par Georges Hervé dans son article: «Le Chinois Tchong-A-Sam à Paris», Revue de l'École d'anthropologie de Paris, Xème année, 1909, pp. 171-179. Les notes sont de Hervé, sauf indication contraire.

Le Chinois Tchong-A-Sam, capturé par un navire corsaire français, fut ramené à Paris et pris en charge par Eustache Broquet, instituteur, qui apprit lui-même, nous dit Hervé, la langue chinoise pour entrer en contact avec lui. Protégé par Bonaparte, Tchong-A-Sam fut embarqué à bord du Naturaliste le 19 octobre 1800, afin «qu'il fût rendu à sa patrie, à sa famille, qu'embarqué à bord de nos vaisseaux, il y fût traité comme officier... Heureuses les nations où de pareils soins sont accordés à l'étranger malheureux! Béni soit le chef ainsi généreux et bienfaisant! 152 »

152 F. PÉRON, Voyage de découvertes aux Terres australes, Paris, Imprimerie nationale, 1807 , p. 11. 
Quelque temps auparavant, Jauffret avait offert à Tchong-A-Sam un exemplaire de son livre: Les Charmes de l'enfance et les plaisirs de l'amour maternel 153. "Ce livre, notait Jauffret 154 , si analogue aux mœurs de la Chine, lui fut une preuve de l'intérêt qu'il m'avait inspiré. Il fut sensible à cette attention, quoiqu'il ne pût rien entendre à l'ouvrage. »

[120]

Les deux documents qui suivent, le premier de Jauffret, le second de Leblond ont été lus lors de la séance publique de la Société des observateurs de I'homme le 18 thermidor de I'an VIII. Le rapport du citoyen Broquet dont il est ici question n'a malheureusement jamais été retrouvé.

LA SOCIÉTE DES OBSERVATEURS DE L'HOMME ne peut remplir avec succès le but utile qu'elle s'est proposé dans ses travaux, qu'en multipliant les recherches et les observations sur un sujet si intéressant à connaître et si peu connu jusqu'ici. Elle doit être plus jalouse de recueillir des faits, plus portée à applaudir au mérite modeste de ceux qui se bornent à en amasser, qu'ambitieuse de présenter des systèmes ou de les admirer. Un auteur systématique cherche moins la vérité que l'illusion. Il prend pour la réalité les chimères d'une imagination exaltée, et lors même que la force de la vérité l'entraîne, il se raidit contre elle, si la direction qu'elle lui donne l'écarte de la route qui lui fut tracée par son orgueil.

Les réflexions que je fais à cet égard s'appliquent naturellement au mode à suivre en étudiant un individu fort intéressant à connaître, mais fort difficile à observer. Je veux parler du jeune Chinois qui est actuellement à Paris, et avec lequel un observateur intelligent entre-

153 L.-F. JAUFFRET, Les Charmes de I'enfance et les plaisirs de l'amour maternel, Paris, Moutard, 1791.

154 Cf. G. HERVÉ, «Les premiers cours d'anthropologie», Revue anthropologique, vol. XXIV, 1914, pp. 255-276, où est publiée la 11ème Leçon de Jauffret sur I'Histoire naturelle de l'homme, consacrée aux peuples asiatiques et à la civilisation chinoise en particulier. 
tient déjà des relations assez intimes pour en obtenir des réponses précieuses.

La Société entendra sans doute avec intérêt le citoyen Broquet faire part des efforts qu'il a fait pour venir à bout d'établir une communication d'idées entre le jeune Chinois et lui. Ces efforts successifs, que le succès le plus complet commence à couronner, sont d'autant plus dignes de fixer votre attention qu'ils ne sont le fruit d'aucune combinaison systématique. Ils méritent d'être recueillis et conservés. Le citoyen Broquet, instruit du désir que la Société des observateurs de l'homme a témoigné de les connaître, s'est empressé de venir lui en faire part. Il aurait amené au milieu de vous son élève et son ami, si ce dernier, retenu au lit par un petit mouvement de fièvre avait eu la liberté de le suivre. Le citoyen Broquet, craignant d'oublier quelque fait intéressant dans le rapport succinct qu'il va vous faire, a bien voulu promettre de faire hommage à la Société de ses propres notes, pour la mettre dans le cas de recueillir celles qui lui paraîtront plus utiles. Il a proposé, de plus, de faire au jeune Chinois, devant des commissaires nommés par la Société, toutes les questions sur lesquelles une réponse pourrait être jugée utile.

\section{Rapport sur le jeune Chinois qui est actuellement $\dot{a}$ Paris}

La Société des observateurs de l'homme ne pouvait être indifférente au bruit public qui annonçait l'existence à Paris d'un Chinois.

Elle éprouva naturellement le besoin de recueillir, dans une telle occasion, des matériaux précieux pour la connaissance d'un peuple qui se tient religieusement isolé du reste de l'univers. Elle nous a chargés, Jauffret et moi, des premiers renseignements; elle a voulu que nous puissions la mettre à portée de juger ce qu'elle doit espérer ou tenter, et de déterminer la série d'observations que peut fournir, au physique, au moral et à l'intellectuel, le représentant de la plus ancienne et de la plus nombreuse des associations existantes. 
Nous ne nous dissimulons pas combien sont encore imparfaits les résultats de nos recherches; mais nous croyons avoir fait ce que permettaient les circonstances, et la Société pourra estimer le moment où il sera convenable d'en faire davantage.

La difficulté la plus grande, sans doute, était d'obtenir quelque point de contact avec le jeune étranger. Et s'il nous est permis d'épancher ici des sentiments amers, ce sera moins sur le malheur que prépare à cet enfant d'un autre monde, l'abandon où il est resté que sur la déshonorante indifférence qui, dans le sein d'une nation policée, n'a laissé produire aucun acte de l'antique hospitalité dont nos pères s'honoraient. Mais fallut-il un autre motif d'intérêt? Combien il était puissant, celui de mettre en action une grammaire vivante de la langue près de laquelle échoue tout l'orgueil de nos savants! Aucun d'eux ne sent-il donc plus cet aiguillon impérieux, ce besoin de savoir qui ne demande que des occasions, que des sujets d'étude?

$\mathrm{Ce}$ que les savants, ce que les initiés dans les langues orientales n'ont pas fait, un homme zélé, éloigné par lui-même de toutes les faveurs du gouvernement, obligé de dérober à ses propre occupations tous les instants qu'exigeaient ces nouveaux rapports; un homme enfin qui avait tout à surmonter pour cette entreprise, a osé se dévouer. Le citoyen Broquet doit recevoir nos éloges, puisqu'il a pris sur lui tout ce que les premiers abords ont eu de difficile.

Figurez-vous, estimables confrères, l'infortuné abandonné dans un hôpital après six mille lieues de traversée, arraché à ses compatriotes pour ne plus voir, ne plus entendre que des êtres insensibles et au moins sourds aux expressions de sa douleur et de ses besoins. Voilà I'homme que, sur son lit de misère, le citoyen Broquet a voulu rendre au bonheur, à la société.

Le premier, il a cherché à deviner ses désirs, à lui faire soupçonner qu'on pouvait encore lui être utile, qu'on saurait apprécier ses maux, et qu'on se flattait de les adoucir. 
C'est au citoyen Broquet lui-même qu'il appartient de retracer la succession de tentatives et d'essais qui l'ont amené à ce degré d'intimité par lequel il est à la fois l'interprète et l'élève de celui qui est aussi jaloux d'entendre que d'être entendu.

Dans les observations dont le citoyen Broquet enrichira nos Mémoires, vous serez surtout pénétrés de la sainte retenue à laquelle il a soumis ses opérations. Ce [122] n'était pas à l'intelligence, c'était au cœur qu'il fallait tout adresser. Le sentiment est de tous les pays, de tous les âges. Honneur, honneur à la délicatesse qui a subjugué cette âme trop justement aliénée par ses malheurs! Honneur surtout à la longue patience qui se pressait moins de recueillir des faits que d'accoutumer la plante à la nouvelle vie qu' un autre ciel lui préparait !...

Deux voies se présentaient: enseigner à l'étranger le français: apprendre de lui sa langue. Le citoyen Broquet a préféré celle-ci, parce que, fort de ses dispositions personnelles à l'étude des langues, soutenu par le concours de différents dictionnaires et ouvrages grammaticaux, il était bien plus sûr d'accélérer les rapprochements.

La Société verra dans quel cas il vaut mieux prendre l'autre voie pour étudier les développements de l'esprit qui arrive à la lumière par la force même de ses rayons. Mais elle sentira probablement que ces développements ne pouvaient avoir d'intérêt réel qu'en raison de l'intelligence déjà manifestée dans les premiers dialogues du Chinois et de son laborieux interprète.

En effet, s'il eût été de cette classe obscure, plus ignorante en Chine que chez nos peuples policés, quelle utilité y eût-il eu à recommencer son éducation? Telle philosophique et telle perfectible qu'on eût espéré de la rendre, elle ne nous eût pas fait acquérir sur la Chine des connaissances que l'individu n'en eût pas rapportées. Nous aurions eu un homme de plus, mais cet homme n'aurait rien eu de chinois.

Du moment, au contraire, où nous pouvons retrouver en lui les traces précieuses d'une éducation soignée et l'aptitude qui en résulte pour saisir et comparer les notions nouvelles, nous sommes sûrs que le parallèle de nos mœurs avec celles dont il est lui-même le résultat, donnera lieu à des rapprochements aussi nombreux que piquants. 
Il était d'autant plus essentiel de reconnaitre, avant tout, si c'était réellement un Chinois que nous avions à notre disposition, que I'on a toujours regardé comme un phénomène de supposer un Chinois hors de ses antiques limites 155 . Tous ceux qui, journellement, s'embarquent pour négocier aux îles de la Sonde, au Bengale et même au Golfe persique, appartiennent à la horde confuse qui peuple Macao: mélange de diverses nations, parlant un méchant portugais, et n'ayant du grand peuple que l'habit et le stupide asservissement aux Quum de tous les ordres.

Notre voyageur nous a bien expliqué qu'il était de Nankin, et seulement établi à Canton pour le commerce ${ }^{156}$. D'ailleurs, la forme même de sa tête, dont le crâne se recule sous un angle de 40 degrés, est aux yeux des naturalistes le caractère de la race mongole. Notre confrère Cuvier a bien précisé cette observation dont il enrichira nos Mémoires.

[123]

C'est encore un caractère national que le plaisir avec lequel il s'est vu revêtir d'habits chinois, au cabinet des Antiques et chez le citoyen Sylvestre ${ }^{157}$.

Il s'appelle Tchong-A-Sam. Tchong serait le nom de famille: $A$ une sorte d'article de Sam, terme numéral annonçant qu'il est le troisième en ordre de progéniture.

Le frère qui l'accompagnait dans l'expédition, et pour lequel il témoigne une grande vénération, s'appelle Tchong-A-Gui, avec le terme numéral de deuxième. Ce Tchong-A-Gui a vingt-cing ans. Le nôtre n'en a que vingt-trois. Il est marié à une femme de dix-neuf. Mais, ce qui

155 Cependant, comme le notait Le Magasin encyclopédique, dans un article sur le jeune Chinois (6e année, T. III, pp. 100-102), « les excursions des particuliers chinois en Europe ne sont pas aussi rares qu' on se l'imagine vulgairement; elles se font surtout par le moyen des Anglais. "

156 Avec sa famille composée de quatre frères et deux sœurs (Ibid., T. III).

157 Le cabinet des Antiques, dont Millin de la Société des observateurs, était le conservateur. Le citoyen Sylvestre de Sacy, professeur d'arabe à la Bibliothèque nationale. 
nous ramène à la piété filiale si chère aux Chinois, ce n'est pas pour sa femme, c'est pour sa mère que ses yeux se sont baignés de larmes quand il a parlé du retour de ses compatriotes, au milieu desquels on le redemanderait en vain. Et prenant aussitôt le ton douloureusement maternel, il s'écrie : « Manque A-Sam ! manque A-Sam! »

Cette famille est vouée au commerce; il paraît que les Anglais avaient déterminé les deux frères à venir négocier directement en Europe, avec l'assurance de les ramener aussitôt. Du thé, de l'encre de la Chine, des éventails, des colliers odoriférants, voilà tout ce qu'il a encore été possible de reconnaître dans l'énumération des marchandises qui formaient leur pacotille. L'expédition était composée de dixsept Chinois, dont quatre négociants, trois ouvriers (tailleurs et cordonniers) et dix matelots; dans le surplus de l'équipage se trouvaient quatre Portugais et soixante Anglais. Tous ont été faits prisonniers par un de nos corsaires, qui les a débarqués à Bordeaux 158. Les Chinois y ont figuré plusieurs mois. Nous croyons même avoir démêlé qu'ils y ont donné le spectacle d'une course de chevaux et d'une lutte à la chinoise, qui a causé aux Bordelais le plus grand plaisir.

De Bordeaux à Orléans, d'Orléans à Valenciennes, les prisonniers ont été confondus jusqu'au moment de l'échange. A-Sam, s'étant trouvé malade, a été abandonné dans I'hôpital d'où, par de nouvelles mutations, il a été transféré jusqu'à celui du Val-de-Grâce, où il reste sous la police militaire.

Sa santé, toujours faible, nous a paru souffrir beaucoup plus du désagrément de sa position que de causes intérieures; et, dans le fait, pouvons-nous croire qu'un homme né dans l'aisance ne soit pris du désespoir lorsque, sans aucun moyen d'exprimer ses besoins et de connaître le sort qui lui est réservé, il se sent éloigné de tout secours et de toute consolation? Si la vie des hôpitaux n'est pour la majeure partie de nos soldats qu'une vie de souffrances et de privations, que doit-elle être pour un individu qui ne participe à nos mœurs, à nos usa-

158 La traversée avait été de près d'un an, sans relâche nulle part. Les prisonniers restèrent environ sept mois à Bordeaux, et, après un séjour de quatre mois à Valenciennes, on les fit, à l'exception d'A-Sam, passer en Angleterre (Magasin encyclopédique, be année, T. III). 
ges, que par les contrariétés que nous lui faisons éprouver et par l'esclavage, les jeûnes auxquels nous le réduisons?

[124]

Cet état habituel de peine était un grand obstacle aux opérations intellectuelles dont il eût été si précieux que rien ne ralentit l'enchâ̂nement. Comment suppléer à ce calme de l'esprit, si nécessaire pour tous les hommes et surtout pour ceux qui, comme A-Sam, sont séparés de la société par toute l'organisation de la société ?

Toutes les figures que nous lui avons montrées ont servi de texte à autant de leçons qu'il donnait au citoyen Broquet sur la manière de prononcer les noms et d'exprimer les idées. Celles que nous savions copiées d'un manuscrit du cabinet Bertin lui ont paru très vraies: quelques-unes, copiées de Du Halde et dont nous-mêmes savions nous méfier, ont attiré sa juste critique, entre autres celle de l'empereur: non pas qu'il voulût se glorifier de l'avoir jamais vu, personne nous exprima-t-il avec une sorte de respect, personne ne le voit. Il y a ici quelque contresens, car, dans une autre occasion, il a décrit la manière dont l'empereur paraît en palanquin dans les cérémonies.

Un de ces contresens s'est expliqué avec un peu de réflexion: nous lui montrions un planisphère chinois; il en reconnut la forme, en fit entendre l'usage et en prit occasion de dire, avec une sorte de contentement, qu'à la différence de notre pays, dans le sien les étoiles paraissaient toujours. Ce toujours n'est bien évidemment qu' une opposition à la fréquence des nuages qui, dans nos climats, voilent la majeure partie de nos nuits.

Nous lui avons présenté aussi une grande pancarte, par-dessus laquelle brochaient plusieurs configurations en encre rouge. Le titre seul et quelques caractères de la première colonne lui ont paru intelligibles. Nous avons lieu de croire que cet écrit est un placard de défenses s'affichant à un poteau, et dont le titre, l'intitulé sont en chinois, le reste en quelque idiome de nation dépendante. Les signes rouges sont les paraphes de chaque mandarin qui a ainsi apostillé ou sanctionné cette défense, en lui imprimant le caractère qu'on ne peut méconnaître sous peine de la vie. 
Nous n'avons pu asseoir aucune idée nouvelle sur l'écriture chinoise. Il n'y a pas de doute que chaque clef ne soit un composé de plusieurs signes rappelant chacun leur radical ; mais comment le composé de ces radicaux finit-il par donner une idée implexe, s'exprimant par un son monosyllabique?

Il épelait, pour ainsi dire, 8 ou 10 éléments combinés dans une clef, prononçait ensuite le son unique pou, dont Fourmont donnait en marge l'indication: mais il ne pouvait y attacher d'idée. C'était le mot servus, pris comme politesse de style épistolaire. Ce contraste mérite d'être approfondi et peut contenir une des questions les plus intéressantes sur la langue chinoise.

Nous ménagions comme l'exercice le plus intéressant celui du petit instrument qui sert aux Chinois pour leur calcul : au moyen de petites boules d'ivoire enfilées 7 par 7, par colonnes, dans une espèce de châssis. Dès que nous lui avons montré l'instrument, il a répété le nom sous lequel nous le connaissions de souan pan, et il a affecté aux colonnes ceux de la numération. Puis il s'est mis à exécuter tous les [125] calculs, comme la Chambre des comptes les faisait, avec des jetons qu'on relève de 5 en 5 ou de 10 en 10.

En Chine donc, le calcul décimal est dans toute sa pureté, et le même souan pan, en affectant telle ou telle colonne à celle des unités satisfait aussi bien aux milliards qu'aux millièmes parties. Mais il ne s'est pas borné à répondre à nos questions, à effectuer les calculs que nous lui donnions. Il a aussi voulu éprouver nos connaissances; il nous a dicté une addition, l'a figurée avec le souan pan et l'a articulée en français.

Cette articulation n'est pas la moindre difficulté à surmonter pour son éducation : car, de même que beaucoup de ses intonations nous paraissaient très pénibles à former, les nôtres lui présentent des nuances qu'il ne peut saisir.

Toute en monosyllabes, la langue chinoise se refuse aux sons liés, et non seulement d'une syllabe à l'autre A-Sam laisse un intervalle mais, dans une syllabe, il ne peut émettre assez rapidement les sons que nous appelons consonnes doubles. Il place entre deux une voyelle, 
et la plus analogue à celle qui doit suivre. Ajoutez à cela la substitution des consonnes fortes aux faibles, et vous verrez le mot grand devenir caland; gros, kolos; français, falançais. Nous récrierions-nous encore lorsqu' un gn, un tch plus ou moins guttural, un ia plus ou moins modulé, nous échappant dans sa prononciation, nous fera confondre des idées très séparées pour lui?

Nous ne pouvons finir sans consigner quelques-unes de ses réponses sur une matière que nous avions bien peu de données même pour effleurer:

«Y a-t-il longtemps que Confucius est mort?

" Cien, çien, çien, çien, et tout plein de çien»; çien est le nombre 1000.

«Où est-il ? »

Il nous a figuré un buste, nous a montré que ce buste se retrouvait partout et qu'on le saluait avec beaucoup de respect. "Les Chinois, ajoutait-il, sont tous des hommes de Confucius. »

Nous aurions dû faire observer qu'il a saisi dans la traversée quelques mots portugais et quelques mots anglais: mais la manière dont il les défigure ne permet pas de s'en servir pour communiquer avec lui. Laissons plutôt le citoyen Broquet devenir chinois; admirons dans le jeune A-Sam la délicatesse avec laquelle il redresse la prononciation et fait répéter les mots mal rendus: voyons dans cette attention une double preuve de la bonne éducation d'A-Sam. En effet, en même temps que son oreille est assez susceptible pour ne pas se contenter de sons grossiers ${ }^{159}$, il a ce dernier degré de sociabilité qui craint de manifester quelque apparence de reproches ou d'offenser l'amourpropre des autres.

159 A-Sam, d'après Le Magasin encyclopédique (be année: T. III) avait quitté Nankin à dix ans, et appris à lire, à écrire et à compter durant les trois dernières années qu'il y avait passées. Il sait tracer en chinois les caractères correspondants à tous les mots qu'il emploie et qu'il profère, et sait lire les caractères chinois qu'on lui présente. 
[126]

Il est de l'essence de la Société des observateurs de l'homme, il est du devoir de tous ceux qui aiment leurs semblables, de désirer pour A-Sam une position plus heureuse. Quand on pense aux faibles secours qui suffiraient pour assurer son existence dans une autre maison publique, on ne peut douter que bientôt I'humanité, I'hospitalité, l'amour des sciences, ne réparent les injures qui leur sont faites dans la personne de cet étranger. Puissent les regards de la Société hâter ce moment et jeter, en attendant, quelques douceurs sur le sort de ASam, qui est pour nous Res sacra, miser! 
Aux origines de l'anthropologie française. (1994) Édition revue et corrigée par les auteurs, 1993.

\section{Texte 6}

\section{Rapport fait à la Société des observateurs de l'homme sur l'enfant connu sous le nom de sauvage de l'Aveyron (1801)}

\section{Par Philippe Pinel}

\section{Retour à la table des matières}

Ce rapport fut découvert et publié par Georges Hervé en 1911 dans son article: "Le Sauvage de l'Aveyron devant les observateurs de I'homme», Revue anthropologique, vol. XXI. 1911, pp. 383-398. Le rapport lui-même est reproduit de la page 411 à la page 454 de ladite revue.

Ni l'article de Hervé, ni le mémoire de Pinel ne sont mentionnés par Lucien Malson dans son livre Les Enfants sauvages, mythe et réalité (Paris, U. G. E., 1964), où sont pourtant reproduits et commentés les rapports d'Itard sur le «sauvage de l'Aveyron », non plus que par Robert Castel dans son ouvrage L'Ordre psychiatrique, l'Age d'or de I'aliénisme, (Paris, Minuit, 1976) qui se réfère souvent et amplement aux travaux de Philippe Pinel. 
En plus de l'édition que nous en avons proposée en 1978, le rapport de Pinel est repris in extenso dans l'ouvrage qu'Harlan Lane a consacré à l'enfant sauvage de l'Aveyron (Paris, Payot, 1979 ; édition originale américaine, Harvard University Press, Cambridge, Mass., 1976) puis, en deux parties - correspondant à sa lecture par Pinel lors de deux séances de la Société des observateurs de l'homme (octobre 1800 et mai 1801) - par Thierry Gineste dans son livre Victor de I'Aveyron. Dernier enfant sauvage, premier enfant fou (Paris, Le Sycomore, 1981 ; réédition revue et augmentée, Paris, Hachette, 1993) qui, par l'importance des matériaux réunis et analysés, est à ce jour l'ouvrage de référence sur cette affaire.

[128]

C'est en 1799 que la Société des observateurs de I'homme fut informée de l'existence de Victor de l'Aveyron, et que Louis-François Jauffret adressa la lettre suivante aux administrateurs de I'hospice de l'Aveyron, à Saint-Affrique, où il fut recueilli :

Paris, 9 pluviôse an VIII

Citoyens,

S'il est vrai que vous avez maintenant dans votre hospice un jeune sauvage de douze ans trouvé dans les bois, il serait bien important pour le progrès des connaissances humaines qu'un observateur plein de zèle et de bonne foi pût en s'emparant de lui, en retardant de quelque temps sa civilisation, constater la somme de ses idées acquises, étudier la manière dont il les exprime, et voir si la condition de l'homme abandonné à lui-même est tout à fait contraire au développement de l'intelligence.

C'est à Paris, par les soins de mon ami et confrère Sicard, instituteur des sourds-muets, et sous les yeux de quelques autres observateurs de l'homme, que ces recherches intéressantes devraient se faire. Elles attacheraient sur le jeune sauvage les regards du public et lui assureraient un sort avantageux. Ce serait donc de votre part une cuvre bien méritoire que de le 
faire conduire à Paris. Sur votre réponse, il vous sera sur-lechamp expédié des fonds, et si, pour hâter son arrivée, vous en faisiez l'avance, ils vous seraient aussitôt remboursés.

\section{Salut et considération,}

\section{L.-F. Jauffret}

Après que le "sauvage de l'Aveyron » eut été conduit à Paris sur ordre du ministre de l'Intérieur, Champagny, à l'institution des sourds et muets (dirigée par l'abbé Sicard), et confié à Itard, médecin de cette institution, la Société des observateurs de l'homme désigna une commission de cinq membres chargés d'observer le jeune "sauvage». Composée de Cuvier, Gérando, Jauffret, Pinel et Sicard elle devint vite le lieu de divergences théoriques, voire de controverses et polémiques, entre Gérando et Pinel notamment, qui mettent en lumière les points sur lesquels portait le débat philosophique et anthropologique de l'époque : l'idée de nature humaine, l'influence de la civilisation, de la société, sur la constitution et le développement de cette nature humaine, la part de l'inné et de l'acquis, etc.

On observe en effet que les conclusions et les interprétations d'Itard vont dans le sens de celles de Péron, c'est-à-dire tendent à démystifier "l'homme de la nature», à considérer que l'homme est inférieur à un grand nombre d'animaux dans le «pur état de nature », cet état le privant, selon Itard, des facultés imitatives qui, dès son plus jeune âge, permettent l'éducation de ses organes, l'apprentissage de la parole, la formation de l'affectivité et le développement des connaissances, permettent en somme qu'il soit un homme dont la nature sociale est ainsi présupposée et affirmée.

Pinel, au contraire, faisant valoir son expérience d'aliéniste à Bicêtre et des comparaisons qu'elle lui permit d'établir entre le développement psychomoteur de [129] Victor de l'Aveyron et de celui observé chez certains de ses patients atteints d'idiotisme ou de démence, fait preuve d'une prudence et d'une réserve plus grandes, tant dans les observations que dans les conclusions qu'il tire du cas «Victor»; il soutient même que le défaut de sociabilité et d'intelligence constaté, loin d'être l'effet d'une désocialisation, peut être, à l'inverse, impu- 
table à une arriération mentale, à une affection congénitale qui l'aurait provoquée et qui aurait donc rejeté Victor du monde des hommes, soit qu'il fut abandonné par ses parents peu après sa naissance, soit qu'il se fût égaré dans les bois 160 .

Du tableau ainsi tracé et de la comparaison établie avec ses propres patients, Pinel n'infère pas de correspondances entre le sauvage et le débile ou l'idiot, entre le défaut de sociabilité, d'insensibilité morale et l' «état de nature», entre la mentalité des peuples dits primitifs et celle de Victor, lequel en serait un témoin en quelque sorte « à domicile». Pinel invite justement à se garder de généralisations abusives, d'assimilations hasardeuses faites à partir d'un cas qu'il persiste à croire pathologique: «Veut-on prendre, dit-il, le mot de sauvage dans une acception plus déterminée et seulement d'après les relations les plus authentiques des voyageurs qui nous ont fait connaître les premiers degrés de civilisation des divers Peuples de la terre? Les objets de comparaison sont alors beaucoup plus fixes et plus précis, mais il n'en peut résulter aucune sorte de lumière relativement à l'enfant de l'Aveyron, puisqu'on ne trouve presque aucun point de conformité entre lui et les individus qui composent les hordes sauvages. Il ne faut, pour s'en convaincre, qu'une simple lecture d' un recueil qu'on vient de publier sous le titre de Voyages chez les peuples sauvages, ou l'homme de la nature 161 ».

Au-delà du diagnostic de Pinel, s'esquisse cependant un débat dont la portée théorique, et même politique, n'est pas négligeable en cette

160 Interprétation qu'avancera Claude Lévi-Strauss dans Les Structures élémentaires de la parenté (Paris, Mouton, 1967, p. 5) sans pourtant se référer explicitement au rapport de Pinel : «Mais, écrit-il, il apparaît assez clairement des anciennes relations que la plupart de ces enfants [« sauvages»] furent des anormaux congénitaux, et qu'il faut chercher dans l'imbécillité dont ils semblent avoir à peu près unanimement fait la preuve, la cause initiale de leur abandon, et non, comme on le voudrait parfois, son résultat. »

161 Ouvrage de C.-F. BABIÉ dont le compte rendu fut publié dans Le Magasin encyclopédique, $\mathrm{n}^{\circ} 17,1800, \mathrm{pp} .138 \mathrm{sq}$. 
époque de fondation de sciences qu'on appellera plus tard humaines et sociales 162 .

Selon Pinel donc, « idiot » et parce qu'idiot, Victor aurait été rejeté dans l'état de nature: «idiot» et parce qu'idiot, Victor devenait un patient, un assisté. Pour Itard et, à sa suite, Gérando, Victor était au contraire « normal » à la naissance, mais c'est l'état de nature dans lequel il était tombé accidentellement (et traumatiquement) qui avait érodé ses facultés de départ et, par conséquent, l'avait rendu «idiot». De là l'«attitude missionnaire» d'Itard qu'Octave [130] Mannoni a bien mise en évidence dans sa critique de l'attitude du médecin des sourds-muets : normal au départ, Victor devenait un assisté rééducable ${ }^{163}$. La relation thérapeutique se transformait en relation pédagogique. Si Pinel laissait encore une place au regard décentré, insistait sur la méthode comparative, suggérait une observation in situ (comme du reste le fit Jauffret dans sa lettre à l'hospice de SaintAffrique), Itard, quant à lui, s'engagea moins vers une observation participante $d u$ "sauvage» que vers une observation qu'on pourrait appeler intervenante, faisant preuve parfois d'une sorte de "terrorisme pédagogique " qui le conduisit à négliger aussi bien les facultés acquises par Victor dans le "pur état de nature » (son exceptionnelle "habileté motrice») que ses jeux, ses "enfantillages" avec la gouvernante, Mme Guérin, qui s'occupa de lui, et à réprimer grâce aux saignées l'éveil de sa sexualité. "Le monde sérieux d'Itard, écrit Mannoni ${ }^{164}$, une fois la récréation (avec Mme Guérin) terminée, c'est un monde où tout plaisir est une récompense, toute peine une punition, sinon ils sont sans signification. Le désir doit ramener au besoin. 》

La fierté avec laquelle Itard relate et commente le premier rhume que Victor attrapa à la suite de « douches écossaises» qu'il lui fit subir et l'extraordinaire " théorie » qu'il en bâtit, avançant à partir de cette anecdote que la «fréquence et la multiplicité des maladies de l'espèce humaine tiennent moins à la nature particulière de son organi-

162 Pour plus de détails sur ce débat, Voir J. JAMIN, «L'indigent, l'indigène et les Idéologues », Anthropologie et sociétés, vol. 3, n², 1979, pp. 55-80.

163 Cf. O. MANNONI, Clefs pour /'imaginaire ou l'Autre Scène, Paris, Seuil, 1969.

164 O. MANNONI ; op. cit., 1969, p. 191. 
sation qu'à l'influence de la civilisation», que «le premier résultat de celle-ci est de développer la sensibilité nerveuse», et que "l'exaltation de cette sensibilité devient la source de nos maladies, comme celle de nos jouissances $\$ 165$ - argumentation tout à fait proche dans l'esprit de la non moins extraordinaire théorie que Péron échafauda à partir de ses observations sur la force musculaire des « sauvages » de Tasmanie -, tout ceci est désarmant de naïveté mais révélateur de sa démarche et de l'idéologie ethnocentriste qu'elle véhiculait. Il s'agissait de conformer le comportement du «jeune sauvage», malgré son esprit certes, mais également malgré son corps, à celui du civilisé.

La maladie devient ainsi un signe d'acculturation, - l'insensibilité corporelle vaincue implique dans cette perspective que l'insensibilité morale le soit. Le passage de la nature à la culture est alors perçu comme un accroissement de la sensibilité humaine - au sens strict - à son environnement naturel et social. Mais au-delà du signe, la maladie (Itard) et la force ou la jouissance (Péron) renvoient l'une à un appareil technique de détection et de guérison, l'autre à un appareil social et culturel d'apprentissage, toutes deux à un dispositif d'éducation et de rééducation au sens large de la sensibilité.

[131]

Avec Victor, Itard a voulu réaliser un pur chef-d'œuvre pédagogique, croyant, comme ses collègues observateurs de l'homme l'avaient cru au début, que la nature lui « offrait la pure nature à instituer $\gg 166$. Cependant Victor, comme le suggère Pinel, était déjà «structuré » au moment du « contact»; il l'était d'une manière certes différente mais qu'Itard négligea de prendre en considération et d'observer. On a pu ainsi reprocher à Itard 167 de s'être trop polarisé sur l'apprentissage du langage parlé puis écrit, alors que, médecin des sourds-muets, il aurait pu tenter lui-même l'apprentissage du langage gestuel ou chercher à analyser ce langage d'action que Victor utilisait pour exprimer ses besoins et manifester ses désirs...

165 Cité par H. LANE, L'Enfant sauvage de l'Aveyron, Paris, Payot, 1979, p. 107.

166 O. MANNONI, op. cit., 1969, p. 201.

167 H. LANE, op. cit., 1979, p. 171. 
En dépit de la persistance du mythe de l'« enfant nu», venu au monde insensible, indifférencié, vierge de toute culture et de toute structure - un peu comme le sauvage lointain devait l'être aux yeux d'Itard, plus tard à ceux de Péron -, Pinel venait suggérer qu'au moment du «contact» Victor était déjà en possession d'une structure dont la qualification - « idiotisme »-nous paraît moins significative et peut-être moins importante que la démarche qui l'amena, et que l'attitude d'observation, d' « écoute» dirait-on actuellement, qu'elle aurait pu impliquer. Itard, au contraire, d'abord convaincu de la «normalité »native de Victor et de I' "arrêt» de son développement intellectuel, chercha moins à la comprendre, à l' « écouter » qu'à le redresser. Or Victor, dès son retour à la société, était autre que la statue de Condillac. Il était déjà dans la culture, ne serait-ce que par le fait paradoxal il est vrai - qu'il avait été abandonné par ses parents à un âge certainement postérieur à trois ans (Pinel avance qu'il a pu l'être vers huit ou neuf ans), - âge où les projections fantasmatiques parentales, voire sociales, avaient commencé de structurer sa personnalité.

Quoi qu'il en soit, le rapide passage de Victor chez les observateurs de l'homme souleva d'autres problèmes sans doute plus importants que ceux qui étaient apparus au sujet du diagnostic - et du pronostic - qu'il convenait de porter à propos de Victor. L'un d'eux fût certainement d'ébranler les hypothèses anthropologiques des observateurs. Victor n'était certes pas ce sujet d'expérience dont Destutt de Tracy et Jauffret avaient rêvé pour analyser l'origine des idées et établir la généalogie des connaissances humaines: mais les méthodes d'observation utilisées avaient déjà « faussé » le terrain : ni Itard, ni Pinel, ni Gérando ne cherchèrent en effet à observer l'enfant en dehors de l'institution qui l'avait pris en charge ou en dehors d'une relation qui se révéla être moins d'observation que de subordination. Ceci contraste avec l'attitude que Leblond et Jauffret adopteront au sujet du Chinois Tchong A -Sam (cf. supra).

Un autre problème, plus moral sinon plus politique, touchait de près au projet philanthropique, posant notamment la question des moyens et celle, quasi théorique, de l'interaction entre l'observation et l'intervention. D' une certaine façon, [132] Gérando - qui fut et resta un ardent partisan des idées et méthodes d'Itard (cf. infra, son Rapport 
sur le rapport d'Itard) - vit peut-être dans « l'art de s'emparer de la sensibilité [de Victor], de la diriger, de la développer $\$ 168$ un moyen de lever la paralysie des facultés intellectuelles qui, frappant le jeune Victor, frappait aussi, à un degré moindre, les déshérités de la société: un moyen d'amener ces derniers « sur notre territoire »169, même si la dureté des procédés et la pédagogisation intense risquaient d'altérer l'observation. "Il ne s'agit pas seulement, écrit Gérando 170, comme on le suppose, de procurer du travail à l'indigent; il s'agit souvent de lui donner l'éducation au travail à tout âge; c'està-dire de lui en inspirer le goût, de lui en faire acquérir la capacité et contracter l'habitude. Il ne s'agit pas seulement, comme on le suppose, d'atteindre un but économique [....], il s'agit surtout d'atteindre un but moral [...]. Il y a peu à espérer comme spéculation du produit d'une telle industrie; mais il y a beaucoup à attendre de ses effets sur les moeurs des pauvres, alors même que la spéculation serait infructueuse. "Pour Gérando, I'observation et la connaissance de l'indigent doivent être utiles: elles doivent permettre d'intervenir, de soulager, d'améliorer. Mais pour lui, plus précisément, elles doivent aussi permettre de choisir, de classer, de mesurer l'opportunité de l'intervention. En 1824, dans Le Visiteur du pauvre 171 , se souvenant sans doute des Considérations... et du cas Victor, Gérando tentera de concilier I'observation et l'intervention dans I'« inspection sociale", - celle qui devrait amener à distinguer les vrais des faux besoins "en pénétrant à l'intérieur du pauvre», "en le bien visitant». Ainsi l'inventeur de I'observation participante met-il en place une véritable technologie du besoin qui « impliquera une surveillance continue de la famille, une pénétration intégrale de tout le détail de sa vie $172 »$.

168 Cf. le rapport de J.-M. DE GÉRANDO reproduit infra ainsi que H. LANE, op. cit., 1979, p. 78.

169 H. LANE, op. cit., 1979, p. 130.

170 J. -M. DE GÉRANDO, De la bienfaisance publique., Paris, Renouard, 1939, T. II, p. 287.

171 J.-M. DE GÉRANDO, Le Visiteur du pauvre, Paris, L. Colas, 1924 (réimpression Jean-Michel Place, Paris, 1990).

172 Cf. J. DONZELOT, La Police des familles, Paris, Minuit, 1977, p. 68. 
Avec Itard, suivi en cela par Gérando, l'indigent n'est plus ce sujet d'observation qui accepte de l'être et auquel Jauffret songeait; il devient un sujet d'inspection, objet de surveillance et non plus de connaissance. Leurs réflexions, en gauchissant quelque peu celles des observateurs de l'homme, annoncent l'analogie qui sera faite plus tard entre éducation et civilisation, - celle qui sous la plume de Bertin et Le Play connaîtra tout au long de la deuxième moitié du XIXe siècle une grande audience 173. Quelques décennies après, ce sera en effet au tour de l'indigène de devenir un sujet d'inspection et un objet d'éducation.

[133]

On serait tenté, enfin, de voir dans le destin de Victor de l'Aveyron la métaphore du destin intellectuel que l'histoire française des idées réserva aux Idéologues. A peine Victor eut-il commencé d'apprendre à parler et de franchir le ténébreux passage qui sépare l'état de nature de l'état de culture qu'il se tut puis disparut, comme disparurent ceux pour qui la recherche du degré zéro de la pensée et de l'expérience sociale était devenue une exigence épistémologique.

UN ENFANT TROUVÉ errant dans les bois d'un de nos départements du Midi et réduit à l'état le plus agreste, fixe depuis quelque temps l'attention publique. L'intérêt naturel qu'on inspire toujours à cet âge joint à l'idée d'un abandon absolu et des périls extrêmes qui en ont été la suite, a fait remonter à l'histoire des autres enfants réduits dans différentes époques au même degré d'isolement. On s'est félicité d'être le témoin d'un de ces phénomènes qui ne s'offrent qu'à des distances très éloignées et sur lesquels on n'a encore que des rapports vagues et infidèles. Les savants d'un certain ordre, ceux qui s'occupent spécialement de l'histoire de l'entendement humain, se sont applaudis de pouvoir étudier le caractère primitif de l'homme et

173 Cf. G. LECLERC, L'Observation de I'homme, Paris, Seuil, 1979, p. 174. 
de connaître la chaîne particulière d'idées et de sentiments moraux qui sont indépendants de l'état social. Mais bientôt on a vu disparaître cette brillante perspective par les bornes très circonscrites des facultés morales de cet enfant et sa privation absolue de l'usage de la parole. Plusieurs mois de séjour dans l'établissement national des sourds et muets n'ont laissé apercevoir aucun progrès sensible, aucun signe de perfectibilité et quelque circonspection qu'il faille mettre dans les présages, rien ne paraît annoncer un avenir plus heureux. Il nous a donc fallu abandonner la marche suivie par notre confrère Bonnaterre qui a considéré cet enfant dans son rapport avec de prétendus sauvages sur lesquels nous n'avons que des notions très incomplètes ou des relations exagérées, et on s'en est tenu à la simple exposition et au rapprochement de faits faciles à constater et à vérifier. On a remarqué que le prétendu sauvage de l'Aveyron avait plusieurs attributs, plusieurs traits caractéristiques communs avec plusieurs enfants dont les fonctions des sens ou les facultés morales sont plus ou moins lésées et qui sont condamnés à végéter tristement dans nos hospices, comme non susceptibles d'aucune culture; dès lors la marche à suivre est simple: c'est de commencer par décrire l'état moral actuel du prétendu sauvage, de faire succéder des notices détaillées sur un certain nombre d'enfants dont les fonctions organiques ou les facultés morales sont plus ou moins lésées. L'autre partie du mémoire, qui sera réservée pour une autre séance, sera destinée à faire le rapprochement des faits antérieurement exposés, et à rapporter les inductions qui en sont la suite naturelle. 


\section{I - État actuel des fonctions organiques et des facultés morales de l'enfant connu sous le nom de sauvage de l'Aveyron.}

Ses yeux ne semblent se fixer avec une certaine attention que sur les objets de sa subsistance, ou sur les moyens d'évasion qui lui sont offerts lorsqu'il est dans une chambre; dans toute autre circonstan$\mathrm{ce}$, il laisse errer vaguement sa vue et sans montrer une intention directe excepté pour tout ce qui excite un moment sa surprise. Une camée fixa un jour sa vue, et il appliqua sur elle ses lèvres pour la baiser, mais, l'instant d'après, on la lui montra vainement à plusieurs reprises. Se mirer dans une glace, comme il l'a fait quelquefois, est-ce s'élever au-dessus de l'instinct animal, et ne voit-on pas faire la même chose à un chat, à un singe? Sa vue est si peu exercée, qu'il ne paraît pas distinguer un objet en peinture de celui qui est en relief, et il porte également sa main sur l'un comme sur l'autre pour le saisir.

Quoique privé de l'organe de la parole, il est loin d'être affecté de surdité. Qu'on pousse un cri derrière lui, qu'on produise un bruit intense, il se retourne aussitôt, mais ce n'est que pour la première fois et lorsque la surprise se joint à l'impression faite sur l'organe de l'ouie: car si ensuite le même bruit se répète, il n'y fait plus attention. Une impression plus légère suffit pour le faire regarder en arrière, lorsqu'elle se rapporte à ses besoins physiques, comme lorsqu'il entend casser une noix: mais il est entièrement insensible à toute sorte de musique, et il est sur ce point beaucoup au-dessous de plusieurs individus renfermés dans nos hospices. Doit-on craindre même de dire que les éléphants ont à cet égard sur lui un avantage marqué ?

L'odorat est le sens sur le témoignage duquel il s'en rapporte le plus pour juger des bonnes ou mauvaises qualités des aliments, et c'est là sans doute une suite de la vie agreste qu'il a menée dans les bois. Qu'il ouvre une armoire, qu'il y trouve de la viande, ou des racines potagères cuites ou crues, il les flaire aussitôt avant de les porter à la bouche et, s'il est à portée d'un brasier, il y jette ces comestibles, les 
retire aussitôt, et les flaire de nouveau avant de les manger. Mais cette préparation grossière des aliments est-elle autre chose que l'effet d'une habitude automatique et contractée depuis qu'il vit dans la société ? On pourrait supposer que l'odorat est en lui très délicat et très cultivé si on ne savait point d'ailleurs qu'il est d'une saleté dégoûtante, et qu'il fait ses ordures dans sa couche même, ce qui semble le mettre au-dessous de l'instinct de presque tous les animaux, soit sauvages, soit domestiques.

La suite de ses progrès dans le choix et la préparation grossière de certains aliments, à mesure qu'il a été témoin des procédés suivis dans les cuisines, montre moins une sorte de culture dans l'organe du goût qu'une imitation automatique de ce qu'il a vu faire, imitation provoquée par les besoins physiques.

Suivant les premiers rapports qu'on a eus sur son état, il ne mangeait que des pommes de terre, des châtaignes crues et du gland; il a vu ensuite qu' on faisait [135] cuire les pommes de terre, et, depuis cette époque, il s'est borné à une ébauche grossière de ce procédé, c'est-à-dire qu'il s'est contenté de les mettre un moment sur la braise et de les retirer aussitôt. Cette sphère s'est encore agrandie pour lui, et il a appris a manger du pain de seigle, du potage, des légumes, des noix, des pommes de terre à demi brûlées, enfin de la viande crue ou cuite. On peut voir, dans la notice historique qu'on a donnée sur sa vie, la série des progrès successifs que son instinct pour sa subsistance a faits jusqu'à ce jour : mais ces limites sont encore très étroites, puisqu'il se borne à fouiller dans l'armoire de la cuisine, et que, sans aucun discernement pour les viandes crues ou cuites, il les porte indistinctement sur la braise, les enlève au même instant, les flaire et les porte à sa bouche. Son industrie n'a pas pu même s'élever jusqu'au point de couper du pain avec un couteau, et c'est un effort suprême et une combinaison inouie de force et d'adresse qui semble le confondre puisqu'il en laisse toujours le soin à une autre personne.

On a dit avec raison que le tact est le sens de l'intelligence, et il est facile de voir combien il est imparfait dans le prétendu sauvage de l'Aveyron. Il est loin de consulter cet organe pour juger des diverses formes des corps, et d'appliquer industrieusement les phalanges de ses doigts autour d'eux pour mieux les palper; il montre, au contraire 
beaucoup de gaucherie dans la manière dont il saisit avec la main les divers comestibles dont il use; ses doigts restent allongés, et l'organe du tact absolument sans action. Il est par conséquent très éloigné de faire servir cet organe pour rectifier les erreurs de sa vue, puisqu'il ne paraît pas distinguer un objet peint sur une surface plane d'un objet saillant et en relief, et puisque en même temps qu'il porte sa main sur un objet pour le saisir, il détourne ailleurs sa vue, ou la laisse errer de côté et d'autre, sans aucune intention directe. On remarque donc en lui une sorte de dissonance entre l'exercice de la vue et celui du tact, et c'est là un caractère que je remarque dans les hospices, parmi les enfants sans intelligence.

Quel autre moyen peut-on avoir de juger de la nature des idées d'un individu de l'espèce humaine, que par des gestes d'un certain ordre, certaines inflexions de la tête et du tronc, ou bien l'usage de la parole? Or l'enfant dont nous parlons est dépourvu de tous ces avantages extérieurs, puisqu'il ne peut parler, et que tous ses gestes et toutes ses inflexions du corps sont insignifiants, ou bien ils se rapportent simplement à ses moyens de subsistance. Dès lors, comment peuton s'assurer s'il a des idées d'une certaine nature, et n'est-on point fondé à présumer qu'il n'a que celles qui sont relatives à l'instinct purement animal ? N'est-on point porté à faire les mêmes conjectures par la manière incomplète dont les organes de ses sens sont affectés par les impressions des agents extérieurs? Il paraît que sur tout ce qui ne se rapporte point à sa subsistance ou à ses moyens d'évasion cet enfant ne conserve aucune idée, ou que, dépourvu d'attention, il n'a que des idées fugaces, et qui disparaissent aussitôt qu'elles sont produite.

Dans quelles bornes étroites n'est point d'ailleurs renfermée la faible combinaison d'idées qui se rapportent à sa nourriture ou aux moyens de vivre dans l'indépendance! [136] Que quelqu' un lui arrache une pomme de terre qu'il tient entre ses mains, il s'approche de lui pour la lui enlever à son tour: mais que cet autre monte sur une chaise pour mettre la pomme de terre hors de sa portée, le prétendu sauvage n'a pas même l'instinct de monter sur une chaise voisine pour s'élever au niveau de l'objet, et il ne s'y détermine qu'après qu'on lui en a donné l'exemple. C'est-à-dire qu'il ne paraît agir que par une imitation 
automatique. Quand il est enfermé dans une chambre avec d'autres personnes, il se rappelle très bien qu'il faut tourner la clé de la serrure dans un certain sens pour ouvrir la porte: mais, depuis plusieurs mois que je l'observe, il n'est pas encore parvenu à savoir imprimer le léger mouvement de rotation à la clé de la serrure, et, étonné de la haute difficulté de l'entreprise, il conduit une personne vers la porte pour lui faciliter sa sortie.

On pourrait attribuer à une forte réminiscence, ou à un essor d'une imagination vive, ces accents aigus, ces éclats de rire immodérés qui se renouvellent brusquement par intervalles, sans aucune cause connue, et qui animent parfois les traits de son visage : mais je puis assurer qu' on observe le plus souvent ces rapides élans d'une hilarité vague et délirante, dans plusieurs enfants ou adultes tombés dans l'idiotisme, et renfermés dans nos hospices 174 : ce sont des saillies vives et spontanées, qui se renouvellent soit le jour, soit la nuit, sans aucune cause connue, et je les regarde depuis longtemps comme des accès passagers de manie et d'extravagance, et quelquefois même comme le partage d'une absence totale d'idées, comme j'en donnerai des exemples dans la suite de ce mémoire. Rien ne peut faire soupçonner, dans ces accès momentanés, aucune réminiscence, aucune expression d'une sensation agréable dont l'imagination soit encore bercée.

Les affections morales dont il paraît doué sont encore très bornées, et ne s'étendent guère au-delà du plaisir que lui donnent des aliments de son goût, ou bien des mouvements de colère qu'il manifeste lorsqu'on les lui enlève ou qu'on l'irrite. N'est-ce point une faible ébauche de sentiment que le léger sourire qu'on obtient quand on lui fait des prévenances?

174 On sait que Philippe Pinel devint le personnage clef du mythe de fondation de la psychiatrie en France, mythe formé autour de la « libération des chaînes » des aliénés de Bicêtre, en l'an II de la République. Sur ce point, voir les analyses et interprétations de M. FOUCAULT, Histoire de la folie à l'âge classique, Paris, Pion, 1961 ; et, s'agissant de ce mythe, les mises au point historiques et épistémologiques de G. SWAIN, Le Sujet de la folie. Naissance de la psychiatrie, Toulouse, Privat, 1978 ; et de J. POSTEL, Genèse de la psychiatrie. Les premiers écrits de Philippe Pinel, Paris, Le Sycomore, 1981. 
Les organes sexuels sont encore sans développement, et il est entièrement étranger à la violence des désirs qui se manifesteront peutêtre à l'époque de la puberté. Il serait peu sage de vouloir juger des circonstances de cette époque orageuse, et du degré d'influence qu'elle pourra exercer sur les facultés morales. Cette période de la vie offrira sans doute un objet piquant pour un esprit observateur, mais en se bornant à l'état présent qui est beaucoup moins problématique, tout annonce que cet enfant est très peu susceptible de s'affectionner même pour les personnes qui lui rendent de bons offices, et comment peut-on séparer ces marques [137] d'affection d'avec les dispositions favorables qu'il manifeste pour tout ce qui se rapporte à sa subsistance?

Nous venons de remplir le simple rôle d'historien, et nous nous sommes renfermé dans l'exacte exposition des faits, pour donner une idée précise des facultés intellectuelles et affectives de l'enfant connu sous le nom de sauvage de l'Aveyron. Avant de nous permettre aucun jugement ultérieur, aucune sorte d'induction, nous allons rapporter, comme objet de comparaison, les traits principaux de plusieurs enfants ou adultes de l'un et l'autre sexe, détenus dans les hospices, à titre d'un état plus ou moins complet d'idiotisme ou de démence. .

\section{II - Notices sur plusieurs enfants ou adultes dont les facultés intellectuelles ou affectives sont plus ou moins lésées.}

Les bornes de ce mémoire ne peuvent permettre que de donner de simples notices de plusieurs enfants, ou plutôt de plusieurs infortunés d'une organisation vicieuse ou mutilée, dont on a recueilli avec soin les histoires particulières, et nous allons d'abord parler des enfants mâles, dont l'état peut offrir des rapprochements plus ou moins marqués avec celui de l'enfant de l'Aveyron.

Un des premiers est privé de l'usage de la parole par un vice physique. C'est-à-dire que sa langue est très courte, et qu'il ne peut absolument $s$ 'en servir pour articuler aucune syllabe, il ne fait entendre qu'un son guttural et obscur: mais d'ailleurs il paraît plein d'intelli- 
gence, et on ne remarque aucune sorte de lésion dans ses facultés morales.

À côté de cet enfant, on peut en citer un autre qui est sourd et muet, et qui, sans avoir eu aucun maître, rend ses idées d'une manière plus ou moins incomplète par des gestes; ce dernier aussi est plein de discernement et susceptible de culture. On voit que M. Sicard doit le réclamer comme entrant dans son domaine.

Un troisième enfant, âgé de neuf ans, est l'image même de l'idiotisme ; il rit ou pleure par une pure imitation automatique, et il n'est sensible qu'aux besoins physiques. Il fait servilement tout ce qu' on lui commande, et ne répond que par oui et par non, et sans discernement, aux questions qu'on lui propose. Sent-il l'aiguillon de la faim, il demande des aliments, et il s'oppose aux efforts qu' on fait pour les lui enlever : mais, sans prévoyance pour l'avenir, il ne met rien en réserve.

Un quatrième enfant, âgé de dix ans, porte tous les caractères d'un albinos: peau fine et blanche, cheveux et sourcils blancs comme neige, les yeux colorés d'un rose pâle, et très sensibles à la lumière: le globe de l'œil, de côté et d'autre, est très saillant, et dans une mobilité continuelle. Cet enfant jouit d'ailleurs des autres fonctions des sens, et ses idées sont bornées aux objets de première nécessité.

Un cinquième enfant, ou adolescent, âgé de seize ans, possède aussi un entendement très borné, et qui n'excède point le cercle des besoins physiques; des attaques réitérées d'épilepsie ont porté l'atteinte la plus funeste à ses facultés morales; il [138] s'isole sans cesse des autres enfants, et passe sa journée à jouer seul avec de petits cailloux. Il jouit cependant d'un faible degré de mémoire, relativement à sa nourriture ou aux traitements durs qu' on lui fait éprouver.

Un sixième adolescent, âgé de dix-neuf ans, et attaqué d'épilepsie paraît au contraire entièrement privé de la mémoire sur certains objets, et oublier avec la même facilité les mauvais traitements comme les bons offices qu'on cherche à lui rendre. Sous d'autres rapports, il a quelque faible degré d'intelligence, et il lie l'idée de ses besoins avec celle des objets propres à les satisfaire: il connaît même la valeur de certaines pièces de monnaie, et dans quelques circonstances il est très irascible, mais il reçoit aveuglément toutes les impulsions qu'on lui 
communique: il articule faiblement les sons, et si on lui dit de chanter il répète éternellement le même couplet comme une machine automatique, à moins qu'on ne l'oblige de cesser ; il ne distingue point encore la différence des sexes.

Un septième adolescent, âgé de vingt et un ans, et attaqué aussi d'épilepsie, mais doué de l'usage de la parole, est remarquable par son inertie apathique, la pâleur de son teint, et une physionomie sans expression. Réduit un jour à pleurer dans un coin, il fut interrogé sur la cause de ses pleurs, et il répondit qu'il n'en savait rien: un instant après, il pousse des éclats de rire en voyant un autre enfant faire des gambades. Tout objet lui est indifférent s'il n'a du rapport à sa nourriture, et, s'il ne se sentait pressé par la faim, il resterait toujours assis ou couché, et dans une attitude immobile. L'avenir est d'ailleurs pour lui comme s'il ne devait point exister, et on ne remarque en lui aucun témoignage de prévoyance.

Je me hâte de passer à d'autres objets de comparaison, pris parmi quelques jeunes filles de l'hospice de la Salpêtrière.

L'une de ces filles, âgée de sept ans, annonce au premier aspect tous les attributs de la santé et de l'intelligence, couleurs vermeilles, cheveux et sourcils noirs, regard vif et animé; elle fixe les objets avec un air d'assurance et une sorte d'attention; mais elle est absolument privée de la parole, et ne fait entendre par intervalles qu'un son sourd et guttural. Entièrement insensible aux caresses comme aux menaces et dans une sorte d'état de stupidité, même pour les besoins physiques elle reçoit les aliments qu' on lui donne sans manifester un air satisfait et les laisse enlever sans aucune opposition. Elle ne rit jamais, et si on la pince, ou qu' on la blesse, elle crie et pleure, mais sans chercher à écarter l'objet nuisible. La seule nuance d'un sentiment agréable qu'elle a manifestée, a été lorsqu' un autre enfant a fait retentir dans son oreille le son d'un chalumeau.

On peut mettre presque sur la même ligne d'idiotisme une autre fille, âgée de dix ans: constitution délicate, visage coloré, le regard vif et animé. Elle fut attaquée de convulsions dès sa naissance, et elle 
a été toujours privée de la locomotion. Chaque jour elle a des accès convulsifs d'environ un quart d'heure, et elle exerce des mouvements si singuliers avec le tronc et les membres qu'on ne peut mieux les [139] comparer qu'à ceux d'un pantin. Elle est insensible aux menaces comme aux caresses, ne distingue personne, ne met aucune opposition, ne témoigne aucune répugnance quand on feint de lui enlever ses aliments; elle laisse échapper par intervalles des éclats de rire immodérés et sans cause, des élans passagers d'une gaieté délirante; elle ne peut articuler aucun son ni prononcer aucune syllabe quoique sa langue ait ses dimensions et sa mobilité ordinaires, elle pousse seulement des sons confus par instants, mais quelques gestes qu' on emploie, quelques objets qu'on lui présente, elle ne donne aucun signe de sensibilité ni d'intelligence, et tout annonce en elle une absence totale d'idées.

Une troisième jeune fille, âgée de onze ans, peut être encore mise au niveau des deux précédentes. Elle avait été bien portante jusqu'à l'âge de sept ans, et paraissait douée de tous les attributs de l'entendement qu'on peut attendre de cette période de la vie. La deuxième dentition donna lieu bientôt après à des convulsions, et, dès lors, elle perdit l'usage de la parole et le libre exercice des fonctions intellectuelles. Des attaques d'épilepsie, qui continuent encore, ont entraîné les changements les plus remarquables; elle a un air d'étonnement et ses yeux sont presque toujours dirigés au hasard; réduite à une sorte d'engourdissement, elle reste toujours accroupie dans son lit, la colonne vertébrale un peu pliée, et les membres dans un état de flexion. Lorsqu' on la contrarie, elle pousse un cri aigu, et fait avec son bras un mouvement automatique, mais en frappant l'air au hasard, et sans aucune intention directe. Elle ne paraît, d'ailleurs, avoir aucune réminiscence, et le sentiment confus de la vengeance cesse avec celui de la douleur. Elle paraît entendre les sons, mais elle ne peut prononcer aucune syllabe, et est entièrement privée de ]'usage de la parole.

Je ne dois point omettre de parler d'une autre épileptique âgée de quatorze ans, qui ne peut prononcer aucune syllabe, quoiqu'elle conserve les fonctions de l'ouie: elle met une vive résistance aux efforts qu' on fait pour lui enlever ses aliments, mais elle semble tout oublier aussitôt qu'ils sont éloignés de sa vue. Il lui arrive souvent de pousser des cris aigus sans aucune cause, de se livrer à des éclats de rire im- 
modérés et à une explosion brusque d'une gaieté délirante, mais elle ne témoigne aucune sensibilité pour les bons offices qu' on lui rend. Ce qui marque surtout un état de stupidité, c'est qu'elle se salit de la manière la plus dégoûtante, et qu'elle ne manifeste aucune répugnance de se vautrer dans ses ordures.

On peut placer à un degré un peu plus élevé trois autres jeunes personnes, dont les facultés morales offrent cependant des lésions manifestes.

La première, âgée maintenant de vingt ans, annonce, au premier aspect, tous les attributs d'un entendement sain: petite taille, teint brun, regard animé, cheveux noirs et épais. Son égarement semble tenir à un amour malheureux ; elle prononce souvent le nom de Debreuil, mais sa raison est tellement troublée qu'elle applique indistinctement ce nom à toute personne, soit homme, soit femme, qui se présente à sa vue; elle passe avec la rapidité de l'éclair d'un langage tendre et affectueux aux [140] invectives les plus grossières; elle parle nuit et jour, et ses affections morales, même les plus disparates, se succèdent ou s'alternent sans ordre, sans suite, sans aucune cause connue.

La deuxième dentition a produit encore un effet plus profond sur une autre personne, maintenant âgée de quarante ans. Elle fut alors attaquée de convulsions, ce qui fut suivi de la perte presque totale des fonctions de l'entendement. Son extérieur, d'ailleurs, se rapporte à son état moral ; elle répond tour à tour oui ou non à la même question, avec un sourire niais, et toute la sphère de ses connaissances semble se borner à satisfaire ses premiers besoins Elle paraît d'ailleurs insensible aux mauvais traitements comme aux prévenances qu'on lui fait. On lui demandait un jour si elle voulait sortir des loges; elle répondit qu'elle voulait être écartelée : c'est qu'elle répétait automatiquement cette dernière phrase, qu'elle venait d'entendre d'une de ses compagnes.

Je placerai enfin en dernière ligne, et à un degré au-dessus de l'enfant de l'Aveyron, une personne qui a maintenant vingt-huit ans, et dont la mère éprouva la frayeur la plus vive au moment de l'accouchement. Elle reste constamment à la même place, sans pouvoir presque articuler aucun son, quoique les organes de la parole ne manifestent aucune lésion physique. Elle prononçait autrefois la voyelle a, seule, et 
ce n'est que par des essais réitérés qu'elle est parvenue à faire entendre les voyelles $e, 0$; mais il n'a pas été possible d'en faire autant pour les voyelles $i, u$. Un élève avait été chargé d'essayer de lui faire articuler quelques syllabes, en lui faisant examiner la position et les mouvements qu'il fallait donner aux lèvres et à la langue. Mais on n'a pu parvenir, après des essais multipliés, qu'à lui faire prononcer les syllabes pa, ba. Elle obéit d'ailleurs servilement à tout ce qu'on lui commande, sans distinguer si ses actions sont sages, extravagantes ou absurdes. Une sorte d'habitude, contractée depuis longtemps par la crainte, lui fait éloigner avec soin toute sorte d'ordures, et personne n'est d'une propreté plus recherchée. Au défaut de l'usage de la parole, les organes de la voix sont doués d'une justesse rare, quoique les sons ne soient formés que par la voyelle a, elle chante quand on le lui ordonne, ou qu'elle entend chanter quelqu'un, et, dans ce dernier cas, elle prend tout de suite l'unisson ou l'octave de la voix qu'elle entend. Si on accélère la mesure, si on change de ton, ou si on appuie sur quelques notes, elle en fait de même, presque avec autant de perfection qu'un écho: mais que, dans ce même moment, elle entende une autre personne chanter un air plus animé, ou qui lui soit plus agréable, elle abandonne le premier et $s$ 'attache à celui qui fait sur elle une impression plus vive. Ses facultés affectives paraissent d'ailleurs oblitérées, et tous les mouvements actifs se rapportent uniquement à sa subsistance.

Je viens d'exposer les faits et les objets de comparaison qui peuvent conduire à la solution de la question proposée. Dans l'autre partie du mémoire, qui sera exposée dans une autre séance, j'examinerai les vérités qui doivent en résulter et j'indiquerai [141] si le prétendu sauvage de l'Aveyron peut être soumis, avec un espoir fondé, à une sorte d'institution et de culture, ou bien s'il faut abandonner cette riante perspective, et s'il faut le confiner simplement dans nos hospices, avec les autres victimes infortunées d'une organisation incomplète et mutilée. 


\section{III - Comparaison entre l'exercice des facultés physiques et morales de l'enfant de l'Aveyron et des enfants réduits à la démence ou à l'idiotisme.}

La vivacité du regard de l'enfant de l'Aveyron est une preuve très équivoque d'un discernement propre à être cultivé, puisque la plupart des idiots des hospices ont les mêmes apparences extérieures et que très peu sont réduits à avoir une physionomie sans expression. Un enfant de sept ans, dont j'ai déjà parlé, et qui est réduit à un idiotisme complet, est remarquable par une extrême vivacité du regard, et de vaines apparences d'un entendement sain: on dirait même qu'il a une sorte d'avantage sur l'enfant de l'Aveyron, puisque son attention n'est pas seulement réveillée par ses moyens de subsistance, mais que souvent il fixe ses doigts et s'amuse à les croiser ou a diversifier leur position avec une apparence d'air méditatif. Une autre fille de vingt ans, réduite aussi à une démence complète, est remarquable par des yeux noirs pleins de vivacité, et une figure très animée. Le peu d'accord qui règne d'ailleurs entre l'exercice de la vue et celui du toucher de l'enfant de l'Aveyron ne doit-il point inspirer une juste défiance? Qu'on lui présente un objet nouveau, soit peint, soit en relief, il y porte quelquefois sa main, mais d'une manière très gauche et de sorte que l'axe de la vision n'est nullement dirigé sur cet objet, au contraire, son regard est errant, et se tourne en général vers la fenêtre ou la partie la plus éclairée de la chambre. C'est ce que j'ai remarqué à plusieurs reprises dans le temps même qu'on traçait son portrait. D'un autre côté, quelques enfants ou adultes idiots savent très bien mettre de l'accord entre l'exercice de la vue et celui du toucher, même pour des objets qui ne se rapportent point à la subsistance. Un d'entre eux passe une partie de la journée à compter de petits cailloux avec une sorte d'attention. Un autre, dont j'ai déjà parlé, conserve des épingles et d'autres petits objets dans un étui qu'il ferme et rouvre à volonté avec toutes les preuves d'une vraie réminiscence. Une autre idiote bien plus avancée que l'enfant de l'Aveyron, est parvenue à calculer 
les nombres jusqu'à seize, mais elle n'a jamais pu concevoir que deux doigts de sa main droite, ajoutés avec deux autres doigts de sa main gauche, formaient le nombre quatre.

La privation de l'usage de la parole, qu'on pourrait prendre comme le résultat d'un défaut d'exercice des organes de la voix, à la suite d'un long isolement, est encore un point de rapprochement de plus avec plusieurs idiots, qui n'éprouvent aucune affection nerveuse. Une jeune fille réduite à l'idiotisme n'est capable d'articuler aucune syllabe, quoique l'organe de l'ouie paraisse dans un état sain, et que la langue exécute librement tous ses mouvements: elle ne fait entendre, non plus que l'enfant de l'Aveyron, que des sons inarticulés et, par intervalles, des cris plus ou [142] moins perçants; il en est de même d'un enfant de sept ans qui jouit de tous les attributs de la santé, mais qui est entièrement privé de l'usage de la parole, et qui laisse seulement échapper de distance en distance un son sourd et guttural. Quel avantage enfin n'a pas sur l'enfant de I'Aveyron une fille de vingt-huit ans, qui semble seulement éprouver une lésion partielle des organes de la voix, et qui n'est parvenue que par des efforts multipliés à prononcer certaines voyelles, répétant d'ailleurs avec une précision et une justesse extrême tous les airs et les cadences qu'on lui fait entendre!

Il existe une disparité remarquable entre l'enfant de l'Aveyron et les idiots des hospices relativement aux objets de subsistance. Ces derniers, sans aucune sollicitude pour l'avenir, sans aucun concours d'efforts, reçoivent à des heures fixes leurs aliments préparés, et sont à cet égard dans une sorte d'état passif, sans connaître, qu'à un très faible degré, l'aiguillon de la faim. L'enfant de l'Aveyron, réduit pendant longtemps à une vie errante et vagabonde, soit dans les bois, soit dans les hameaux, et pressé souvent par une faim dévorante, a dû encore prendre l'habitude de se nourrir des aliments les plus grossiers, et juger d'abord par l'odorat de leur qualité salutaire ou nuisible. Ramené ensuite au sein de la société, son organe du goût a acquis une sorte de développement et lui a appris à rechercher des mets plus soigneusement préparés : de là l'usage progressif du gland, des raci- 
nes, des pommes de terre crues, puis de noix, de châtaignes, de pommes de terre cuites, de légumes, de la viande; mais n'est-ce pas là plutôt le résultat simple du principe de l'imitation que les preuves d'un discernement cultivé ? Ne voit-on pas, même parmi les idiots, des différences qui les mettent les uns au-dessous, les autres au-dessus de l'enfant de l'Aveyron? Un enfant que j'ai souvent sous les yeux possède à un degré si borné l'instinct relatif aux premiers besoins, qu'il ne sait pas même saisir les aliments qui sont à sa portée, quoiqu'il sente vivement la faim; il ne fait alors aucun geste, aucun effort pour les atteindre, et il avance seulement la tête et les lèvres pour les saisir lorsqu' on les lui offre à une très petite distance de la bouche. Un autre enfant réduit à l'idiotisme, mais capable d'articuler les sons, nomme les objets de ses besoins, et marque ses désirs par des caractères extérieurs, au lieu que l'enfant de l'Aveyron n'en a que la simple réminiscence, qu'il ne peut les désigner ni par des sons articulés, ni par des gestes, qu'il ne fait que les reconnaître à la simple vue, c'est-à-dire que la sensation actuelle ne fait que lui rappeler une sensation antérieure. Un autre idiot regarde son dîner avec satisfaction lorsqu' on le lui apporte, et il le mange avec avidité, si même alors on feint de vouloir le lui enlever, il pousse un cri aigu et fait des gestes menaçants: mais aussitôt que son appétit est assouvi, il voit avec indifférence enlever les restes de son dîner, sans marquer aucune prévoyance pour l'avenir, ce qui le met au-dessous de l'enfant de l'Aveyron, qui met des aliments en réserve pour le retour de l'appétit. Enfin on doit mettre dans un degré bien supérieur à ce dernier, une fille idiote qui indique par des sons articulés les objets de [143] ses premiers besoins, qui conserve avec soin les restes de ses repas, et s'irrite même quand on veut les lui enlever, qui sait même que l'argent fournit les moyens de s'en procurer, qui met à contribution les étrangers et apporte à sa fille de service, comme un tribut de reconnaissance, les pièces de monnaie qu'on lui donne: mais toute la sphère de ses connaissances se réduit aux objets de première nécessité.

Ne doit-on pas regarder comme insoluble le problème de l'absence totale ou de la non absence d'idées relativement à l'enfant de l'Aveyron, puisqu'il ne peut s'exprimer ni par des sons articulés ni par des gestes, et que tout ce qu'il fait semble se rapporter uniquement au principe de l'imitation? Peut-on d'ailleurs faire valoir en sa faveur 
le penchant servile qu'il manifeste à imiter ce qu'il a vu faire relativement au choix ou à la préparation très grossière des aliments, puisque plusieurs animaux domestiques sont susceptibles, sous ce point de vue, d'une sorte d'éducation, et que d'ailleurs on trouve cette faculté plus ou moins perfectionnée parmi les idiots des hospices, même pour des objets qui ne se rapportent point à la subsistance?

C'est ce qu'on remarque sur une fille qui parle sans ordre et sans suite, mais qui change brusquement ses propos incohérents au moment qu'un objet nouveau la frappe; elle a même un chant très agréable et elle exécute des danses avec les gestes les plus passionnés. Une autre fille, réduite à un idiotisme complet, est dominée par le penchant le plus marqué et le plus irrésistible pour l'imitation puisqu'elle simule aussitôt tout ce qu'elle voit faire, ou qu'elle répète automatiquement tout ce qu'elle vient d'entendre sans juger nullement des convenances, et sans distinguer si elle parle bien ou mal, elle retient avec une extrême facilité une suite de couplets qu'elle entend chanter une fois, mais sans attacher aucun sens aux paroles qu'elle prononce. La faculté imitative est si entraînante, pour une autre fille dont j'ai déjà parlé, qu'au milieu d'une réponse qu'elle fait elle mêle d'autres mots qu'elle entend prononcer, et qui n'ont aucun rapport avec l'objet primitif dont elle paraissait occupée.

Le penchant à l'imitation est bien plus faible dans l'enfant de I'Aveyron, puisqu'il se borne aux objets de première nécessité, et à des essais informes relatifs à la préparation des aliments ou aux moyens de s'échapper ; encore même est-il circonscrit, sur ces objets, dans des limites très étroites, et n'a-t-il pu encore parvenir ni à couper du pain avec un couteau, ni à tourner la clé d'une serrure dans un certain sens pour ouvrir la porte.

Les éclats de rire immodérés, les accès d'une gaieté vive et folâtre que manifeste l'enfant de l'Aveyron à différentes heures du jour ou de la nuit, loin d'être un signe favorable, ne sont qu'un point de rapprochement de plus qu'on peut établir entre lui et certains idiots des hospices. Une très jeune fille de dix ans dont j'ai déjà parlé, passe quelquefois des heures entières dans des cris inarticulés entremêlés d'éclats de rire, sans qu'on puisse leur assigner d'autre cause déterminante qu'une sorte d'excitation nerveuse et purement automatique. 
Une fille de quatorze ans réduite à un état complet de stupidité et entièrement privée de l'usage de la parole, éprouve par moments ces brusques saillies d'une hilarité vaine et délirante; elle pousse par [114] intervalles, soit le jour, soit la nuit, des cris perçants qui semblent exprimer tantôt le malaise, tantôt une situation agréable.

Dans d'autres personnes affectées d'idiotisme ou de démence, ces accès passagers se prolongent plus ou moins, et prennent même le caractère d'accès maniaques. Une idiote âgée de vingt-six ans éprouve tous les matins une semblable excitation nerveuse de très peu de durée, mais durant laquelle elle peut exercer des actes de la plus haute grande violence. Les faibles nuances de sensibilité que marque l'enfant de l'Aveyron aux prévenances qu' on lui fait, le mettent sans doute audessus de certains idiots des hospices, qui ne paraissent sensibles ni aux menaces ni aux caresses, et qui ne marquent par aucun signe extérieur leur reconnaissance pour les bons offices qu' on leur rend: mais on en peut citer d'autres qui manifestent une sensibilité plus ou moins vive pour ce qu' on fait en leur faveur, et une d'entre elles ne se montre-t-elle pas bien supérieure à cet égard à l'enfant de l'Aveyron, puisqu'elle témoigne un attachement pour la fille de service qui prend soin d'elle, et qu'elle lui rapporte en tribut de reconnaissance les pièces de monnaie qu'elle recueille des personnes qui visitent I'hospice? 


\section{IV - Inductions que font naître les ressemblances et les points de conformité observés entre l'enfant de l'Aveyron et les enfants des hospices réduits à l'idiotisme ou à la démence.}

Un naturaliste distingué a cherché à fixer l'opinion publique au sujet du sauvage de l'Aveyron 175, soit en communiquant le résultat de ses observations sur cet enfant confié quelque temps à ses soins, soit en remontant à des époques antérieures, d'après des relations qu'il a recueillies. Il a cru d'ailleurs devoir rapprocher de ces détails historiques certains fragments qui nous restent sur les relations de quelques autres enfants égarés dans leur bas âge, et retrouvés dans les déserts, loin de la société des hommes. Nous ne ferons point ici la critique de ces recherches, et nous nous bornerons à remarquer seulement que les objets de comparaison que prend ce naturaliste sont très loin d'avoir été transmis avec des détails assez circonstanciés et une exactitude propre à faire cesser les incertitudes. Ce ne sont que des notices vagues recueillies dans des dictionnaires, des journaux, ou des ouvrages de littérature et peut-on citer un seul de ces prétendus sauvages dont l'organisation, les mœurs et les habitudes aient été approfondis et analysés avec un esprit observateur? Quel avantage, dès lors, peut-on retirer d'un semblable rapprochement?

Veut-on prendre le mot de sauvage dans une acception plus déterminée et seulement d'après les relations les plus authentiques des voyageurs qui nous ont fait [145] connaître les premiers degrés de civilisation des divers peuples de la terre? Les objets de comparaison sont alors beaucoup plus fixes et plus précis, mais il n'en peut résulter aucune sorte de lumière relativement à l'enfant de l'Aveyron puisqu' on ne trouve presque aucun point de conformité entre lui et les individus qui composent les hordes sauvages. Il ne faut, pour s'en convaincre,

175 J. BONNATERRE, Notice historique sur le sauvage de l'Aveyron et sur quelques autres individus qu'on a trouvés dans les forêts à différentes époques, Paris, an VIII. (août 1800). Cette notice est intégralement reproduite dans l'ouvrage de TH. GINESTE, op. cit, 1981, pp. 148-173. 
qu'une simple lecture d'un recueil qu' on vient de publier sous le titre de Voyages chez les peuples sauvages, ou I'homme de la nature.

Il a fallu donc reprendre cet objet de recherches sous un autre point de vue, ou plutôt chercher à varier les soupçons d'imbécillité que Bonnaterre avait déjà formés sur l'enfant de l'Aveyron. "Cet état d'imbécillité, dit ce naturaliste, se manifeste dans ses regards, il ne les fixe sur aucun objet; dans les sons de sa voix, ils sont discordants, inarticulés, et il les fait entendre la nuit et le jour: dans sa démarche, il va toujours au trot ou au galop; dans ses actions, elles sont sans but et sans détermination. » Ces soupçons n'ont pu que se confirmer par une considération attentive des mours et des habitudes de cet enfant à diverses époques, et du défaut d'un nouveau développement de ses facultés morales depuis son arrivée à Paris. Ses actes extérieurs bornés à une sorte d'instinct animal, nous ont donné l'idée de le comparer avec les enfants et les adultes dont les facultés morales sont plus ou moins lésées, et qui, incapables de pourvoir à leur subsistance, sont confinés dans les hospices nationaux. L'histoire des uns et des autres a rendu saillants tous les points de conformité qui peuvent exister entre eux. Les objets de comparaison sont ici sous nos yeux, chacun est le maître de venir examiner, étudier, constater les faits sur lesquels nous nous sommes fondés. Quelques-uns des enfants réduits dans nos hospices à un état d'idiotisme ou de démence, sont inférieurs pour les facultés morales, à l'enfant de l'Aveyron; d'autres, comparés à ce même enfant, lui sont égaux ou même supérieurs. N'avons-nous donc pas les plus grands degrés de probabilité pour penser que l'enfant de I'Aveyron doit être assimilé aux enfants ou adultes réduits à un état de démence ou d'idiotisme?

Quelles sont maintenant les circonstances qui ont amené l'enfant de l'Aveyron à cet état d'idiotisme? Ici nous manquons de détails authentiques, et rien ne paraît d'abord pouvoir dissiper à cet égard nos incertitudes. Les parents sont inconnus : l'enfant est privé de l'usage 
de la parole, et de l'avantage de se faire entendre par des gestes. Le passé est pour lui comme s'il n'avait point existé, et nous n'avons aucune autre source certaine de lumières; nous ne pouvons ici nous diriger que par l'analogie des faits, en recherchant quelles sont les causes ordinaires qui produisent la démence ou l'idiotisme dans l'enfance. Or, en excluant de cet état une complication avec l'épilepsie, ou un vice rachitique, ces causes se réduisent à trois points principaux:

$1^{\circ}$ une vive frayeur éprouvée par la mère pendant la grossesse ou l'enfantement:

[146]

$2^{\circ}$ une frayeur ou des convulsions survenues durant l'enfance par des affections vermineuses:

$3^{\circ}$ le travail pénible et orageux de la première ou deuxième dentition.

Rien ne peut déterminer laquelle de ces trois causes a pu agir sur l'enfant de l'Aveyron, et porter une atteinte funeste à ses facultés morales: mais quelle que soit celle des trois qu'on adopte, on peut conjecturer que des parents inhumains ou réduits à un état de disette ont abandonné cet enfant comme incapable de culture, vers l'âge de neuf à dix ans, à une certaine distance de leur demeure, et que l'aiguillon du besoin l'a porté à se nourrir des aliments grossiers que la nature lui faisait trouver sous sa main, sans d'autres moyens de juger de leur qualité salutaire ou nuisible que les impressions faites d'abord sur l'organe de l'odorat, Puis sur celui du goût. Il paraît être ainsi resté errant et vagabond, dans les bois ou dans les hameaux, les années suivantes, toujours réduit à un instinct purement animal, et uniquement occupé des moyens de pourvoir à sa subsistance et d'échapper aux dangers dont il était menacé. 
On connaît tous les autres détails de sa vie depuis qu'il est entré dans la société mais son discernement toujours borné aux objets de ses premiers besoins, son attention uniquement fixée par la vue des substances alimentaires, ou sur les moyens de vivre dans un état d'indépendance dont il a fortement contracté l'habitude, le défaut total de développement ultérieur de facultés morales pour tout autre objet, $n$ 'annoncent-ils point qu'il doit être entièrement rangé parmi les enfants atteints d'idiotisme et de démence, et qu'on n'a aucun espoir fondé d'obtenir des succès d'une instruction méthodique et plus longtemps continuée? 
Aux origines de l'anthropologie française. (1994)

Édition revue et corrigée par les auteurs, 1993.

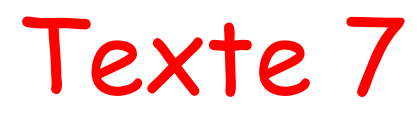

\title{
Présentation du rapport de J.M.G. Itard sur l'enfant sauvage de I'Aveyron (1801)
}

\author{
Par Joseph-Marie de Gérando
}

\section{Retour à la table des matières}

Cet écrit posthume de Joseph-Marie de Gérando a été publié dans les Annales de l'éducation des sourds-muets et des aveugles, Paris, 1848 , tome $5, n^{\circ} 2, p p .110-118$. Il a été lu par l'auteur lors d'une séance de la Société des observateurs de l'homme, précisément le 28 vendémiaire de l'an X (20 octobre 1801). Il a été repris par Thierry Gineste dans son livre, Victor de l'Aveyron. Dernier enfant sauvage, premier enfant fou, Paris, Le Sycomore, 1981 (réédition revue et augmentée, Paris, Hachette 1993).

LORSQUE LE JEUNE ENFANT, appelé le sauvage de l'Aveyron, fut découvert dans les forêts de la Caune, et amené à Paris par le professeur Bonnaterre, cette nouvelle, pendant quelque temps, retentit dans tous les papiers publics, occupa les oisifs, attira près de lui les 
curieux, et fournit le sujet d'une foule de discussions, au moins prématurées, puisqu'elles ne pouvaient se fonder encore que sur des conjectures.

Cependant, le public, qui accourait en foule près de cet enfant, fut fort surpris de ne voir en lui qu'un être à peu près hébété, qui semblait ne rien voir et ne rien entendre, qui ne donnait aucun signe d'attention, qui ne paraissait avoir aucune espèce d'industrie, et l'intérêt fut refroidi aussi promptement qu'il s'était manifesté.

L'esprit de système vint bientôt le frapper d'un nouvel arrêt. Quelques personnes qui veulent voir dans I'homme solitaire I'homme de la nature, selon lesquelles l'état sauvage est non seulement l'état primitif de l'homme, mais encore son [148] état le plus parfait pendant que la civilisation et l'état de société n'en sont au contraire que la dégénération s'indignèrent que l'homme de la nature répondît si peu à leurs espérances, ou redoutèrent qu'une expérience sans réplique vint démentir leur hypothèse. Ils se hâtèrent donc d'affirmer que cet enfant était né imbécile, et qu'une lésion naturelle de ses organes physiques ou de ses facultés morales pouvait seule avoir produit des résultats si contraires à leur attente.

Les esprits superficiels confirmèrent cette sentence. Le sauvage de l'Aveyron ressemblait à un imbécile; donc il était imbécile. Cette induction était à leurs yeux sans réplique; elle était fondée sur les apparences.

Quelques hommes éclairés enfin partagèrent aussi cette erreur, et l'autorisèrent par leur opinion. Les uns, ayant vainement essayé sur lui leurs méthodes d'instruction, supposèrent qu'il était incapable d'être instruit, plutôt que de soupçonner l'insuffisance de ces méthodes elles-mêmes justifiées par d'autres succès dans des circonstances différentes. Les autres rapprochèrent le phénomène offert par l'enfant de l'Aveyron de ceux que l'observation leur avait déjà présentés. Le citoyen Pinel, médecin, qui s'est acquis, par ses traitements sur les aliénés, une si juste réputation, aussi distingué d'ailleurs par sa philanthropie que par l'étendue de ses connaissances, ayant analysé en détail toutes les circonstances dont se composait l'état moral et physique de cet enfant, et les ayant méthodiquement rapprochées de celles qu'il avait remarquées dans un grand nombre de jeunes sujets idiots ren- 
fermés à l'hospice de la Salpêtrière, ayant reconnu des caractères a peu près semblables, se crut fondé à conclure de l'analogie des effets à l'analogie des causes, et, dans un rapport, il annonça qu'il le regardait comme condamné par la nature même à un idiotisme qui laissait peu d'espérance.

Cependant un petit nombre de philosophes s'élevèrent contre une décision si sévère. Ils opposèrent du moins le doute à des assertions qui leur parurent trop précipitées. Ils firent observer qu'avant de conclure de la ressemblance aperçue entre l'état de l'enfant de l'Aveyron et celui des idiots pour cause de lésion dans les organes, il fallait démontrer auparavant qu'il n'y avait pas une sorte d'idiotisme moral, semblable dans ses effets à l'idiotisme physique, et que certaines circonstances extraordinaires, comme un long isolement, une existence brutale, ne pouvaient pas, dans un sujet qui n'avait reçu encore aucune instruction, produire ces habitudes d'inattention, cette paralysie des facultés intellectuelles qu' on remarquait dans le jeune sauvage. Ils rappelèrent à l'appui de ce doute toutes les observations qui prouvent l'influence de l'état de société sur le développement des facultés humaines, et les raisonnements qui montrent l'étroite liaison de nos idées avec nos besoins. Le lieu dans lequel avait été trouvé l'enfant de I'Aveyron, l'ignorance où l'on était de sa vie antérieure, ajoutaient une nouvelle force à cette supposition. Du moins ils assuraient qu'il était imprudent d'affirmer une opinion sur la cause de ce phénomène, avant que l'expérience eût permis de l'approfondir avec plus de soin, et ils conclurent que les hommes sages devaient suspendre leur jugement jusqu'à ce [149] qu'on eût fait sur l'enfant l'essai des véritables méthodes propres à développer son intelligence dans le cas où elle en serait susceptible.

Ils indiquèrent en même temps quelles étaient, à leur avis, ces méthodes. Locke et Condillac les avaient fait pressentir. Ils répétèrent, d'après ces grand maîtres, qu'avant de vouloir faire lier des idées à cet enfant, il fallait les faire naître: que, pour les faire naître, il fallait fixer son attention, et que le seul moyen de fixer son attention était d'intéresser ses besoins. Ils ne conçurent pas qu'on voulût lui faire comprendre des signes, lorsqu'il n'avait encore aucune des notions exprimées par ces signes, lorsqu'il n'avait aucun motif pour re- 
marquer ces signes eux-mêmes, lorsqu'il n'avait aucune méthode pour les comparer. Ils virent donc dans l'art de s'emparer de sa sensibilité, de la diriger, de la développer, le seul moyen de détruire cette inattention habituelle, universelle qui pouvait n'être que l'effet de son indifférence absolue pour ce nouveau monde dans lequel il était encore si étranger. Ils ajoutèrent que ce ne serait que par un exercice très long et très réitéré qu'on pourrait apprécier l'utilité de ces méthodes, parce qu'avant de faire naître de bonnes habitudes on aurait à combattre, à détruire des habitudes contraires, parce que les organes de ce jeune homme, retenus dans une longue inaction, n'avaient plus la souplesse ni la docilité du premier âge.

Les amis de l'humanité se récrièrent aussi contre un arrêt qui pouvait fixer à jamais de la manière la plus fâcheuse le sort de cet infortuné. Ils demandèrent qu'avant de reléguer cet enfant au nombre des idiots, on eût acquis une certitude plus complète de l'impuissance de ses organes. Ils firent sentir qu'un an de délai et d'expérience était bien peu de chose, en comparaison de l'affreuse possibilité de condamner un être sensible à l'existence des animaux.

Leur vœu fut entendu par la bienfaisante administration des sourds-muets et par un ministre protecteur des lumières. Le jeune homme de l'Aveyron fut confié aux soins du citoyen Itard, médecin de I'Institution nationale des sourds-muets, afin que, par la combinaison des remèdes physiques et du traitement moral, il essayât du moins de conserver cet être à la société, avant de le proscrire à jamais de son sein.

Il m'est bien doux de pouvoir aujourd'hui vous apprendre que les espérances des philosophes commencent à se réaliser, et le zèle des amis de l'humanité à obtenir sa récompense. En très peu de temps le citoyen Itard a obtenu des succès qui tiennent du prodige: je les ai vérifiés par moi-même. J'invite ceux de mes collègues qui y attacheraient quelque intérêt, à imiter mon exemple. Les moyens qu'a employés le citoyen Itard, et les progrès par lesquels son élève y a répondu, sont exposés dans un écrit qu'il publie, et dont il vous fait hommage.

Il y rappelle d'abord l'état dans lequel il a trouvé cet enfant, et le témoignage du public est ici d'accord avec le tableau qu'il en trace. Il 
reproduit la suite des inductions qui l'ont conduit à penser que son élève avait en effet vécu longtemps seul au milieu des bois, et que son idiotisme apparent n'était que la suite des habitudes qu'il avait dû contracter dans cette déplorable existence. En partant de cette supposition, [150] le citoyen Itard a fait diverses tentatives pour tirer les facultés morales de l'enfant de l'état d'engourdissement dans lequel elles étaient plongées. Bientôt la méditation des principes que Locke et Condillac ont établis, lui a fait reconnaître que c'était en éveillant sa sensibilité, en la prenant ensuite pour guide, qu'il pourrait arriver à son but. Au lieu donc de contrarier les besoins de son jeune élève, il a cherché à les multiplier, et c'est en épiant le moment où ils se manifestaient qu'il a cherché à s'emparer de son attention. Voici à peu près la marche qu'il a suivie.

L'organe du tact paraissait être entièrement paralysé dans l'enfant : il ne se montrait sensible ni au froid ni à la chaleur. L'odorat et le goût étaient plongés dans un égal sommeil. Des bains chauds et réitérés développèrent bientôt sa sensibilité nerveuse. Les émotions de la joie et de la colère, excitées et ménagées à propos, développèrent aussi sa sensibilité nerveuse. En peu de temps son tact acquit une assez grande délicatesse; il devint difficile dans le choix de ses mets, il y mit une recherche de propreté toute nouvelle pour lui ; il se guida par leurs odeurs.

L'œil de l'enfant était errant et hagard. Il voyait sans doute, mais ne regardait point. Les bruits les plus éclatants paraissaient à peine frapper son oreille, une sonnette, un coup de pistolet né le faisaient point se retourner. Des observateurs superficiels n'avaient pas manqué d'en conclure qu'il était sourd. Le citoyen Itard a sagement aperçu qu'écouter n'est pas la même chose qu'entendre, que la violence d'un son ne suffisait pas pour attirer sur cette impression l'attention d'un être qui n'avait aucun intérêt à le remarquer. Il en a vu une nouvelle preuve dans l'attention que son élève donnait aux moindres bruits qui pouvaient l'intéresser, comme celui d'une noix brisée ou d'une clef tournant dans la serrure. Il en a donc conclu qu'il fallait fixer son oreille, comme son œil, par un intérêt immédiat. Il a réussi par divers artifices très ingénieux, dont le succès a confirmé ses conjectures. 
Cependant de nouvelles habitudes se formaient dans le jeune homme: autant de besoins nouveaux prenaient naissance. La nourriture, I'habillement, le coucher, la promenade, offraient chaque jour des moyens multipliés d'augmenter sa dépendance. En se trouvant placé dans la dépendance de ceux qui l'entouraient, l'enfant a commencé à connaître les affections morales: il s'est vivement attaché à sa gouvernante, il a vu avec plaisir son médecin. Toutes ces affections, tous ces besoins sont devenus, entre les mains de l'instituteur, des leviers puissants qu'il a mis successivement en jeu pour développer son industrie. Ses idées se sont multipliées, se sont liées entre elles. On a commencé à lui créer des amusements ; on a cherché à convertir ces amusements en instruction; on a exercé l'élève à des comparaisons ; on l'a accoutumé à rapprocher les objets de leurs images; on l'a contraint à n'employer dans ces rapprochements que les secours réunis du jugement et de la mémoire. Le citoyen Itard a cru ce moment favorable pour lui apprendre à reconnaître les signes de notre écriture ; il a voulu essayer la méthode qu'on emploie pour l'instruction des sourdsmuets. Il a tracé le nom de l'objet autour de l'image, et effaçant ensuite cette image, il a espéré lier le nom au souvenir de l'objet ; mais la méthode s'est [151] trouvée en défaut. Ici rien de plus philosophique que les réflexions auxquelles ce résultat a conduit l'instituteur, rien de plus heureux que la suite des moyens qu'il a employés pour franchir l'immense distance qui sépare, dans l'histoire du langage, la peinture des objets de leur expression conventionnelle par les caractères de l'écriture. Il faut lire dans l'ouvrage même le détail de ces moyens. Qu'il me soit permis d'annoncer seulement leur effet. Le jeune homme, aujourd'hui, distingue, classe les caractères de l'alphabet: il fait plus : en prononçant sur un ton de voix ordinaire les mots lait, soupe, il va chercher de suite les caractères nécessaires pour ces mots; il les assemble sur une planche, et compose le mot avec toute l'exactitude grammaticale. Chaque jour il acquiert un nouveau terme: ce ne sont, il est vrai, que ceux qui ont un rapport immédiat avec ses besoins, mais ce sont les seuls qu'il soit même permis à un philosophe de lui présenter. Enfin le voici admis non seulement à communiquer avec nous, le voici en possession de nos signes conventionnels. Il a franchi la limite, il est sur notre territoire. 
Sans doute vous n'apprendrez pas sans intérêt cette nouvelle; sans doute vous partagerez la reconnaissance et l'estime qui sont dues au jeune et modeste philosophe qui, en si peu de temps, a opéré un changement si fort au-dessus de notre attente, qui le premier a tenté, a exécuté un genre d'éducation entièrement nouveau, et dont le zèle a surmonté tous les obstacles. Son ouvrage est écrit avec simplicité et candeur ; exempt de tout esprit de système, il y a raconté ses erreurs comme ses succès, il y a exposé ses doutes sur l'avenir en même temps que ses réflexions sur le passé ; il n'y a tu qu'une seule chose, la touchante bienfaisance qu'il a su associer dans mille détails au traitement de son élève. Après avoir lu l'ouvrage, après avoir vu le jeune homme, on ne peut s'empêcher de payer un tribut d'éloges aux soins, au caractère de Madame Guérin, gouvernante de cet élève, qui a secondé si heureusement les vues de son instituteur.

Ici une dernière question se présente. Chacun de nous se demande quel augure on peut tirer des progrès passés de l'enfant en faveur de ses progrès futurs. Sans doute, nous ne nous livrerons pas sans réflexion au besoin de convertir nos espérances en certitude; sans doute, ce doute philosophique qui est la première condition de la vraie science sera placé encore par nous à ces premier résultats. Nous n'affirmerons point d'une manière décisive qu'il n'y ait aucune lésion physique dans les organes du sauvage de l'Aveyron, quoique nous puissions assurer que cette lésion est, dans tous les cas, beaucoup moins sensible qu'on ne l'avait appréhendée: nous n'affirmerons pas même que les seules habitudes morales contractées par cet enfant dans un long séjour au sein des forêts, dans une vie tout animale, puissent entièrement s'effacer. Cependant il est permis de fonder quelque analogie sur les succès déjà obtenus, et d'attendre beaucoup d'une méthode qui a déjà tant produit. Cet enfant est entré dans la société : que ne pourront pas les relations sociales? On a fixé ses organes, pourquoi ne les développerait-on pas par l'exercice? Le doute le plus sérieux naîtrait ici des obstacles que le citoyen Itard a éprouvés dans les efforts [152] qu'il a faits pour amener son élève à l'articulation des sons. Il ne dissimule aucun de ces obstacles, le mot lait et un petit nombre d'autres sont les seuls que jusqu'à cette heure il ait pu lui faire imiter. Cependant, et cette réflexion appartient au citoyen Itard, il faut observer que l'enfant de l'Aveyron ne peut plus avoir dans les organes de 
la parole cette flexibilité qui appartient à la première enfance: une longue inaction doit leur avoir donné une raideur difficile à vaincre. D'ailleurs on ne peut tout faire à la fois. La prononciation, chez les enfants eux-mêmes, est lente et graduelle. Cet enfant n'a aucun intérêt bien prochain à imiter notre prononciation, son attention est partagée par un grand nombre d'autres objets. Attendons que le temps et des essais répétés viennent nous fournir sur ce point, comme sur les autres rapports de son perfectionnement possible, des lumières plus certaines. 
[153]

Aux origines de l'anthropologie française. (1994)

Édition revue et corrigée par les auteurs, 1993.

Texte 8

\section{Mémoire sur l'établissement d'un Muséum anthropologique (1803)}

Par Louis-François Jauffret

Retour à la table des matières

Une version plus élaborée de ce texte aurait été éditée en 1803 à Paris par l'imprimerie de Gillé. Nous n'avons jamais retrouvé la trace de cet ouvrage, qui apparaît pourtant au catalogue de la Bibliothèque nationale.

Une partie du manuscrit fut par contre publiée en annexe et en anglais dans Nicolas Baudin, The Journal of Post Captain Nicolas Baudin, Adélaïde, Libraries Board of South Australia, 1974, 609 p. L'original a été retrouvé par J. -P. Faivre dans le Journal du capitaine Baudin (Archives nationales, Marine, 5 JJ 35, pp. 39-41, in folio).

Le début du texte manque, le Journal s'interrompant de la page 31 à 38 (les notes sont des éditeurs). 
Ces premiers aperçus et ces vues générales 176 pourraient sans doute suffire à la sagacité de nos correspondants, leur faire connaître nos besoins, et les instruire sur les idées fondamentales qui doivent nous diriger dans la formation d'un Muséum spécial où nous espérons par la suite, classer avec reconnaissance et distinction, les preuves de leur féconde et généreuse amitié.

[154]

Cependant, nous croyons devoir ajouter à ces premières réflexions quelques développements qui laissent au moins entrevoir qu'elles n'ont pu être que le résultat d'une longue et laborieuse méditation. En conséquence, nous reprendrons les chefs principaux de division qui viennent d'être énumérés, pour indiquer quels objets, quels produits, quels monuments peuvent $s$ 'y rapporter et figurer dans une collection uniquement consacrée aux progrès de l'étude et de la science de I'homme.

\section{Section I}

De l'homme considéré en simple naturaliste et sans avoir égard aux résultats que peuvent fournir pour en favoriser l'étude, l'analyse de I'anatomiste et celle du métaphysicien.

L'homme considéré de cette manière, objet d'un examen superficiel et ne livrant pour ainsi dire à l'observateur qu'une draperie qu'il faut ensuite écarter et soulever pour arriver à de plus importants résultats. Enfin l'homme étudié en naturaliste présente neuf aspects principaux que j'ai indiqués de manière suivante dans une des tables

176 Manque le début du texte. 
synoptiques qui font partie de mon esquisse d'un cours d'histoire naturelle de l'homme et d'hygiène.

$1^{\circ} \mathrm{L}$ 'homme observé pour connaître les divisions principales de son corps, ses régions ou proportions.

$2^{\circ} L$ 'homme comparé aux animaux afin de déterminer sa place dans l'échelle des êtres vivants et d'après les traits extérieurs qui le caractérisent.

$3^{\circ} \mathrm{L}$ 'homme observé relativement aux différences d'où résultent les races et variétés.

$4^{\circ} \mathrm{L}$ 'homme comparé à l'homme relativement aux différences qui dépendent de dispositions contraires aux lois ordinaires de la nature.

$5^{\circ}$ L'homme comparé à I'homme relativement aux différences qui résultent de l'expression physique des sentiments et du caractère moral.

$6^{\circ} \mathrm{L}$ 'homme considéré relativement aux différences de sexe.

$7^{\circ} L$ 'homme considéré relativement aux différences d'âge.

$8^{\circ} \mathrm{L}$ 'homme qui va cesser d'être ou qui n'est plus.

$9^{\circ} \mathrm{L}$ 'homme livré aux mains qui vont le confier à la tombe, ou retarder par différents procédés l'époque de sa décomposition.

Des squelettes entiers, de différentes grandeurs et où tous les progrès de l'ossification pourraient s'apercevoir et se comparer; des têtes, des crânes, dont on puisse apprécier les formes variées, le type primitif, la déformation, le rapport de leur forme avec l'intelligence, soit par l'angle facial, soit par la proportion entre les aires [155] de la face et du crâne, observées, comme le conseille le citoyen Cuvier, à l'aide d'une coupe longitudinale de la tête.

On peut encore rapporter au premier chef de division différents exemples de monstruosités ou de maladies: les altérations variées de la peau qu'à l'exemple des Allemands il conviendra de dessiner avec une grande fidélité, les ongles coupés ou non coupés et formant dans 
ce dernier cas de faibles instruments d'appréhension: quelques morceaux de téguments remarquables par l'épaisseur et la force des poils qui la couvrent, de fortes callosités, ou la diversité des couleurs que leur donnent différentes pratiques plus ou moins bizarres. Enfin, les pièces des dessins, différents exposés graphiques qui pourront nous faire connaître la hauteur de la taille dans les différents climats, les proportions des membres, les traits propres à l'âge et au sexe, les soins donnés à la vieillesse, ou son état de souffrance ou d'abandon, la conduite envers les mourants, les sépultures considérées sous le double point de vue de la salubrité et des mours, etc.

Tels sont les différents objets qui peuvent éclairer sur un ou plusieurs des aspects que nous venons d'indiquer, et dont la collection et l'envoi seront pour la Société [des observateurs de l'homme] des bienfaits reçus avec gratitude, et des preuves remarquables du zèle de ses correspondants, ou du dévouement et de l'amitié de tout philanthrope qui voudra partager leurs utiles travaux.

Quelques objets importants pourraient encore occuper une place distinguée dans le département de notre Muséum consacré au premier aspect de notre objet spécial d'observation.

Lorsque des rives qu'ils cherchèrent et connurent pour y porter nos arts et le bonheur, lorsque de ces rives lointaines Cook et Bougainville revinrent en Europe, ils y ramenèrent deux Otaitiens, A-outourou 177 et Omai, dont les noms unis à ceux de leurs bienfaiteurs ne seront jamais oubliés. Oserions-nous exprimer le désir de voir notre illustre correspondant imiter cette conduite et faire arriver jusqu'à nous quelques échantillons des variétés humaines qu'il pourra découvrir 178. Ces étrangers seraient surtout accueillis par les observateurs

177 Cf. G. HERVÉ, « Aoutourou ou le Tahitien à Paris», Revue anthropologique, vol. XXIII, 1914, pp. 207-219.

178 Allusion à Nicolas Baudin, correspondant de la Société des observateurs de I'homme, à qui donc ce texte est destiné. 
de l'homme, et si nos soins hospitaliers pouvaient les retenir, les faire consentir en adoptant notre patrie à mettre un espace immense entre leur tombe et leur berceau: leurs restes chéris et conservés dans la suite par le génie de la science et le besoin d'une douce sensibilité viendraient figurer dans notre Muséum, au milieu des attributs de leur patrie dont ils compléteraient le tableau.

[156]

\section{Section II}

De l'homme considéré dans l'exercice de sa sensibilité extérieure, ou sensibilité de relation.

Les instruments qui favorisent ou perfectionnent les principaux organes de cette sensibilité, ceux de l'ouie et de la vision, les machines, les appareils qui donnent une extension plus ou moins grande à leurs sphère d'activité, les instruments plus ou moins nombreux et féconds qui ouvrent des sources nouvelles de plaisir, de bonheur ou d'instruction, enfin tous les monuments qui peuvent faire connaître les différent états de la musique, de la danse, de la sculpture, de la peinture, de la gravure, du dessin, etc. Tels sont les différents objets qui se rapportent à notre second chef de division.

\section{Section III}

De l'homme considéré dans le double exercice de sa moralité et de son esprit.

Les monuments, les objets qui doivent remplir la section de notre Muséum consacrée à ce grand aspect de la monographie humaine sont aussi variés que nombreux. Dans l'impossibilité d'en offrir avec exactitude et complément l'énumération détaillée, je me bornerai à indiquer comme tels les signes hiéroglyphiques ou écrits, les produits qui constatent les différents degrés de perfection dans plusieurs arts utiles ou agréables, les matières employées dans le commerce et les 
échanges, les procédés pour diviser le temps; les moyens variés de tradition, les usages domestiques, les marques du pouvoir et de la subordination: enfin tout ce qui peut instruire sur les rapports des hommes entre eux ou avec la divinité dont le culte varié, la multiplicité, les symboles divers, les attributs et les images sont surtout indiqués par la Société à ses bienveillants collaborateurs.

\section{Section IV}

De l'homme considéré dans 1/'exercice de la locomotion.

Cette fonction ne se bornant pas dans I'homme à de simples déplacements ni à différents modes de transport, il faut réunir à tous les moyens qui peuvent favoriser ces derniers sur la terre et sur les eaux les instruments variés de chasse, de pêche, les armes diverses, les moyens de défense ou d'attaque, la sagaie, l'arc, la fronde, toutes les machines de guerre et de combat.

[157]

\section{Section $V$}

De l'homme considéré dans l'emploi de ses moyens de nutrition, et relativement au fonds de subsistance qu'il a trouvé, créé conquis ou modifié.

La cage du Muséum spécial où doivent se rassembler les différents objets compris sous ce cinquième titre, comprendra principalement différents échantillons de plantes alimentaires, de leurs graines et de leurs fruits, des instruments plus ou moins perfectionnés d'agriculture, des échantillons de différents terrains amendés ou non amendés par la culture, les résultats de leurs analyses, l'analyse des eaux également et ses résultats, les moyens divers de conservation, de préparation et d'assaisonnement des substances liquides et solides qui peuvent nourrir et satisfaire le double besoin de la soif et de la faim. 


\section{Section VI}

De I'homme observé dans ses rapports avec l'atmosphère.

Les objets compris sous ce sixième titre sont nombreux et variés: il faudra surtout distinguer les différents moyens et procédés pour élever la température. Les modes divers et l'usage du feu, les formes variées des habitations soit qu'elles offrent à peine des abris grossiers, des cabanes, des huttes, ou que plus perfectionnées elles se disposent en maisons, les matières employées dans la construction, les pierres diversement taillées, simplement juxtaposées ou maintenues par du ciment, le bois, ses coupes, la réunion des pièces avec ou sans tenons, - différences qui en dégagent de très grandes dans la civilisation; enfin les différents couchers, les nattes, les hamacs, les lits de coton ou de matières analogues, les vêtements, les matières qui les forment, les fourrures, les peaux tannées ou non tannées, les matières pour le tannage ou la teinture, les écorces et les toisons des animaux, ouvrées ou dans leur état de produits bruts, les instruments qui les changent ou les modifient, enfin les costumes plus ou moins simples et variés suivant les différences des peuplades ou les circonstances d'âge et de dignité, etc.

\section{Section VII}

De l'homme considéré dans l'exercice de la reproduction.

Nos correspondants rallieront aisément à ce chef de division, et remarqueront pour les recueillir, les marques distinctives et extérieures du sexe et de la puberté, les signes plus ou moins simples qui cimentent l'union conjugale, les présents accordés ou exigés, la forme des contrats, les moyens plus ou moins grossiers [158] employés pour favoriser l'accouchement, les témoignages monumentaires et traditionnels de la naissance; enfin tout ce qui peut également se connaître pour monuments, de l'éducation physique et morale, des habitudes et de la condition des femmes et des enfants. 
Telles sont les vues générales, les indications que j'ai cru devoir rassembler pour répondre à la demande qui nous est adressée par le capitaine Baudin.

En les offrant à ce célèbre navigateur, je réclame à la fois le produit de ses méditations et le résultat de ses découvertes pour perfectionner et utiliser cette faible esquisse que je n'aurais point osé vous présenter, si mon silence et mon refus avaient pu s'accorder avec mon désir de donner à la Société des preuves de mon zèle et de mon dévouement. 
[159]

Aux origines de l'anthropologie française. (1994)

Édition revue et corrigée par les auteurs, 1993.

\section{Texte 9}

\section{Inventaire général de tous les objets \\ relatifs à l'histoire de l'homme (1804)}

\section{Par François Péron}

\section{Retour à la table des matières}

Le titre complet de cet écrit est: Inventaire général de tous les objets relatifs à l'histoire de l'homme (recueillis pendant le cours de l'expédition ou remis à M. Péron, naturaliste zoologiste du Gouvernement dans cette expédition et présentés par M. Geoffroy et lui à Sa Majesté I'Impératrice Joséphine le 9 prairial an XII).

Considérés comme des objets d'art par les professeurs du Muséum, les objets ethnographiques collectés par Baudin et Péron à l'île Bruny, à la terre de Diémen (Tasmanie), à Port-Jackson et à Timor, ont été dispersés lors du retour de l'expédition en France. Sur les 206 objets inventoriés par Péron, 160, dont un lot particulièrement riche d'objets océaniens offerts par le navigateur George Bass afin qu'ils ornent le Musée de la Société des observateurs de l'homme, tel que l'avait projeté Jauffret, furent donnés à Joséphine Bonaparte, avec le consentement du Muséum d'histoire naturelle. 
Ces collections ont été pillées en 1815 à la Malmaison ou vendues en 1829, après la mort du prince Eugène de Beauharnais. Il n'existe aucun catalogue de cette vente. L'inventaire reproduit ici est de la main de Péron. Il a été retrouvé par E,-T. Hamy en 1906, dans les archives du Muséum du Havre, puis publié pour la première fois par lui dans l'article: «Les Collections anthropologiques et ethnographiques du voyage de découvertes aux terres australes (1801-1804) ) », Bulletin de géographie historique et descriptive, $n^{\circ} 1,1906, \mathrm{pp} .24-34$. Aucune trace de ces objets n'a été retrouvée depuis. (Les notes sont renvoyées enfin de chapitre.)

[160]

1. Bougie bénite de Notre-Dame de Candelaria,

Ténérife protectrice des îles Canaries.

2. Bras d'une momie des Gouanches (anciens habi- Idem tants des îles Canaries).

3. Canne de bois de santal (Santalum album Linn) Timor [2] 179 .

4. Grosse torche de cire dont les principaux chefs Idem malais se font précéder le soir.

5. Sagaie des naturels de l'intérieur de l'île, avec Idem une pointe de fer et un manche de bambou 180 .

6. Sagaie des rois de Timor avec une longue hampe Idem de bois très brun, armée d'une longue pointe de fer assez bien travaillée, laquelle est enchâssée dans un étui de cuivre. Le manche est orné de deux touffes de poil rouge très fin 181.

7. Turban d'un roi malais musulman. Idem

8. Plumet du turban. Idem

179 Les chiffres entre crochets à la suite des descriptions sommaires des objets indiquent leur nombre.

180 Les numéros 5 à 9 avaient été donnés par M. Losfett, alors gouverneur de l'île.

181 On s'en sert plus ordinairement à cheval (F. PÉRON). 
9. Sabre d'un Rajah malais garni d'une belle houppe Idem de poils rouges 182 .

10. Pâte de riz dont les Chinois de Coupang font plu- Idem sieurs petits ouvrages.

11. Pantoufles chinoises en maroquin rouge (1 paire). Idem

12. Pantoufles chinoises en maroquin noir (1 paire). Idem

13. Sac à bétel en forme de ridicule ornée de touf- Idem fes de soie d'un Rajah de Timor (provenant d'Amadima, roi de Suabawa).

14. Boîte à chaux appartenant au sac à bétel $n^{\circ} 13$. Idem

15. Boîte à bétel garnie en étain. Idem [161]

16. Boîte à tabac. Idem

17. Giberne d'un roi malais, garnie de ses cartou- Idem ches en bambou.

18. Bonnet de guerre des naturels de l'intérieur de Idem Timor.

19. Boîtes en feuilles de latanier (sans couvercle) Idem servant à mettre le bétel [2].

20. Boîtes en feuilles de latanier comprimées, rec- Idem tangulaires [5].

21. Boîtes en feuilles de latanier pour tabac et bé- Idem tel, cylindroïdes [5].

22. Boites en bambou pour la feuille de bétel [10]. Idem

23. Boîte en bambou garnie en étain, pour la feuille Idem de bétel.

24. Cuiller en coco dont presque tous les habitants Idem se servent à Timor.

25. Cuillers en corne de bure [3].

Idem

26. Boîtes à chaux pour le bétel [3]. Idem

27. Calebasse ronde et plate servant de vase à eau. Idem

28. Collier de grosses graines violettes qui sert Idem d'ornement à quelques femmes.

29. Ficelle très fine faite avec les fibres des feuil- Idem les de bananier.

182 Il se porte pendu à l'épaule gauche (F. PÉRON). 
30. Espèce de petite boîte fermée, triangulaire, en Idem feuilles de latanier plissées.

31. Boîtes plates quadrangulaires en latanier. Idem

32. Samiote chinois représentant le dieu Fô, en ter- Idem re vernissée (Coupang).

33. Écritoire chinoise en marbre blanc. Idem

34. Écritoire en pierre argileuse très fine et d'un Idem gris verdâtre.

[162]

35. Cuiller en porcelaine brune à l'usage des Chinois Idem de Coupang.

36. Espèce de boîte conique en feuille de latanier. Idem

37. Touffe de cheveux graissés et papilloté avec de Canal d'Enl'ocre rouge (naturels du canal d'Entrecasteaux. trecastaux Péron).

38. Modèle d'un catamaran d'écorce dont se servent Idem les naturels du canal d'Entrecasteaux.

39. Sagaie des naturels de la Terre de Van Diémen. Île Maria

40. Sabres à ricochet [8], naturels du Port Jackson Port Jackson (armes terribles et jusqu'à ce jour complètement inconnues) [Péron].

41. Crochet à lancer la sagaie $n^{\circ} 46$ [5]. Idem

42. Crochet à lancer la sagaie, fait en forme de lan- Idem ce.

43. Casse-tête à pointe [6].

Idem

44. Massue dont l'extrémité très grosse et très Idem renflée est peinte en rouge.

45. Massue terminée par une grosse et forte pointe. Idem

46. Sagaies de trait [5].

Idem

47. Sagaies de main [4].

Idem

48. Hameçon presque circulaire d'un seul morceau Idem de coquille.

49 [5]. Massue sculptée dans toute sa surface, garnie à son extrémité de portions osseuses et présentant sur l'une de ses faces beaucoup de figures très grossières d'un homme armé d'un casse-tête (avec la lune et le soleil à son

Îles des navigateurs 
extrémité).

50. Massue sculptée dans toute son étendue, tron- Idem quée à son extrémité représentant un homme armé d'une massue, un second naturel pareillement armé d'une massue et d'un bouclier et, ce qui peut paraître plus singulier, une espèce d'ancre de navire assez semblable à celle des Européens et vraisemblablement imitée d'eux.

[163]

51. Massue sculptée dans toutes ses parties, mais Idem sans figure particulière (obtusément pointue à une de ses extrémités).

52. Massue sculptée dans toutes ses parties, elle est la plus courte de toutes et la plus renflée vers la pointe.

53. Massue sculptée sur toute sa surface, dentée sur ses bords de distance en distance, plus aplatie, plus étroite que les précédentes.

54. Casse-tête, très gros et très renflé vers son extrémité, qui se trouve en même temps rehaussée de gros tubercules sculptés (et réunis entre eux par une sorte de ligature assez singulière, le manche seul est sculpté).

55. Espèce de rame en forme de hallebarde, d'un Idem bois noir très dur et très pesant, taillée en forme de scie tranchante sur ses bords et qui la rend une arme très redoutable [2].

56. Espèce de petit banc courbe à quatre pieds sur lequel les habitants peuvent reposer leur tête.

57. Filet de pêche en forme de seine, garni d'un bois très léger sur son bord flottant, tandis que l'autre est chargé de galets ou de petites pierres dont quelques-unes sont madréporiques et la plupart volcaniques.

58. Autre seine semblable à la précédente, mais plus Idem grande et faite avec du fil blanc.

59. Espèce de tresse plate, d'un roux jaunâtre et 
qui paraît être faite avec les fibres du brou de coco.

60. Lignes de pêche garnies chacune d'un hameçon Idem dont la hampe est faite avec un morceau de coquille et le crochet avec un morceau d'écaille de tortue 183.

61. Espèce de ceinture de cordes peinte en rouge et Idem noir.

62. Espèce de parasol de tête triangulaire en écaille Idem de tortue avec deux cordons sur les côtés pour le fixer aux oreilles.

63. Espèce de parasol de tête triangulaire et garni Idem de latanier.

[164]

64. Espèce de chasse-mouches.

Idem

65. Statue grossière et très informe représentant une femme.

66. Coco très volumineux entouré d'un treillage.

67. Espèce de cordage quadrangulaire à petits anIdem neaux articulés ensemble, comme nos chaînes de montre, ce qui le rend élastique.

68. Espèce de panier quadrangulaire oblong d'un tis- Idem su très serré, peint en noir.

69. Espèce de double coupe en bois supportée par Idem quatre pieds dont deux sous chaque coupe.

70. Espèce de double portefeuille en écorce, dont la Idem poche intérieure contient les peignes du numéro suivant.

71. Peignes élégamment travaillés, formés d'une douzaine environ de petites baguettes de bambou écartées les unes des autres par un tissu très fin, très serré, établi entre chacune d'elles à la base du peigne.

72. Espèce de poche ou de panier quadrangulaire

Idem

Idem

\author{
Idem
}

Îles des navigateurs

\title{
Idem
}

183 Ce qui suit, de 49 à 101, forme une collection donnée par le capitaine Bass pour le Muséum de la Société des observateurs de l'homme (F. PÉRON). 
aplati, flexible, faite avec un cordage très rude, très raide, dont le treillis est hérissé de gros nouds, sa couleur est d'un brun très foncé.

73. Natte grossière d'une couleur grise uniforme ${ }^{184}$.

74. Grande pièce d'étoffe de plusieurs aunes de lon- Île Sandwich gueur en trois doubles, dont I'un noir, l'autre jaune, le $3 e$ jaune strié de noir.

75. Espèce de natte de la forme d'une serviette ayant les bords profondément dentés.

76. Grande natte jaune d'un tissu très fin.

Îles des navigateurs

77. Natte d'un tissu plus grossier que la précédente, Idem d'une couleur jaune blanchâtre.

78. Rame d'une grande embarcation avec la poignée Îles des Amis ornée d'une belle nacre verdâtre en même temps qu'elle est sculptée avec soin.

[165]

79. Rame d'une petite embarcation avec un simple anneau de sculpture vers son milieu.

Idem

80. Rame en forme de hache très large à son extrémité et très aplatie, tronquée et $n$ 'ayant qu'un seul anneau sculpté vers la poignée.

81. Sagaie renflée en massue vers sa poignée sculp- Idem tée dans ses deux tiers antérieurs, atténuée vers sa pointe et armée de forts aiguillons dans le bas et alternativement opposés par leurs pointes.

82. Habillement jaune à grandes fleurs pennées rouges, la matière en est d'écorce frappée.

83. Trois morceaux d'étoffe d'écorce blanche.

Idem

84. Espèce d'habillement jaune avec des fleurs pennées et de larges bandes anguleuses.

85. Espèce d'écharpe jaune avec des dessins noirs Idem transversalement dirigés.

86. Espèce de banc sur lequel les naturels reposent Idem

184 Les numéros 5 à 9 avaient été donnés par M. Losfett, alors gouverneur de l'île. 
leur tête comme sur un coussin. Cet échantillon

n'a que 3 pieds résultant de la formation elle-

même de la branche d'arbre employée à sa construction.

87. Espèce de tresse plate et noire.

Îles des Navi-

gateurs

88. Espèce de grand vase de bois à quatre pieds, Idem très poli dans son intérieur, peu profond et d'un bois très odorant.

89. Espèce de tabouret à quatre gros pieds courts et très polis ainsi que le siège lui-même.

90. Espèce de sagaie sans aucune sorte de sculpture que de grosses entailles à la plus grosse extrémité.

91. Sagaie extrêmement longue sans aucune espèce de sculpture, atténuée à ses deux bouts, plus renflée vers son milieu; le bois en est très soli (elle est cassée dans son milieu).

92. Sagaie très longue, cylindroïde, sans aucune apparence de sculptures que 24 anneaux de barbillons épais et fort obtuse à sa pointe.

[166]

93. Ornement en forme de hausse-col, très large et très grand, fort adroitement incrusté d'un très grand nombre de grains de cascavèle.

94. Espèce de bonnet d'un tissu d'écorce très dure, Waitao $d$ 'une couleur rousse très foncée, avec des taches noires triangulaires.

95. Hameçon de pêche dont la hampe est de bois et le crochet d'un morceau d'os.

Île Mangea

95 bis. Hameçon de pêche dont la hampe est de bois et le crochet d'un morceau de coquillage.

96. Hameçon de pêche de deux morceaux de coquilIdem lages larges réunis en angle, la nacre de la hampe est d'un beau vert irisidiant.

97. Hameçon de pêche très petit d'un seul morceau de coquille.

Nelle Zélande

Idem 
98. Espèce de sabre de bois avec, sur les côtés, des Idem dents de squale très acérées et très pointues.

99. Espèce de tresse blanche quadrangulaire, tissée Idem d'une espèce de poils très forts.

100. Espèce de hache de pierre.

101. Paquet d'une espèce de tresse plate et rougeâtre ${ }^{185}$.

102. Hache de pierre des naturels de Port du Roi George, singulièrement remarquable par la solidité de la résine qui sert à souder le manche.

103. Petit panier de la jeune Kanaga, fille du Roi de Babao.

[167]

104. Petit panier à eau qui m'a été donné par cette Idem même jeune fille.

105. Deux boucliers des naturels de la Nouvelle Hollande dont un provenant de Bannelou chef des sauvages de Sydney, lequel a été envoyé en Angleterre et présenté au Roi qui l'a comblé de ses bienfaits.

Nelle Hollan-

Idem

Île des navigateurs

Nelle Hollan-

de

Timor

de

185 Ici finit la collection de Bass. 


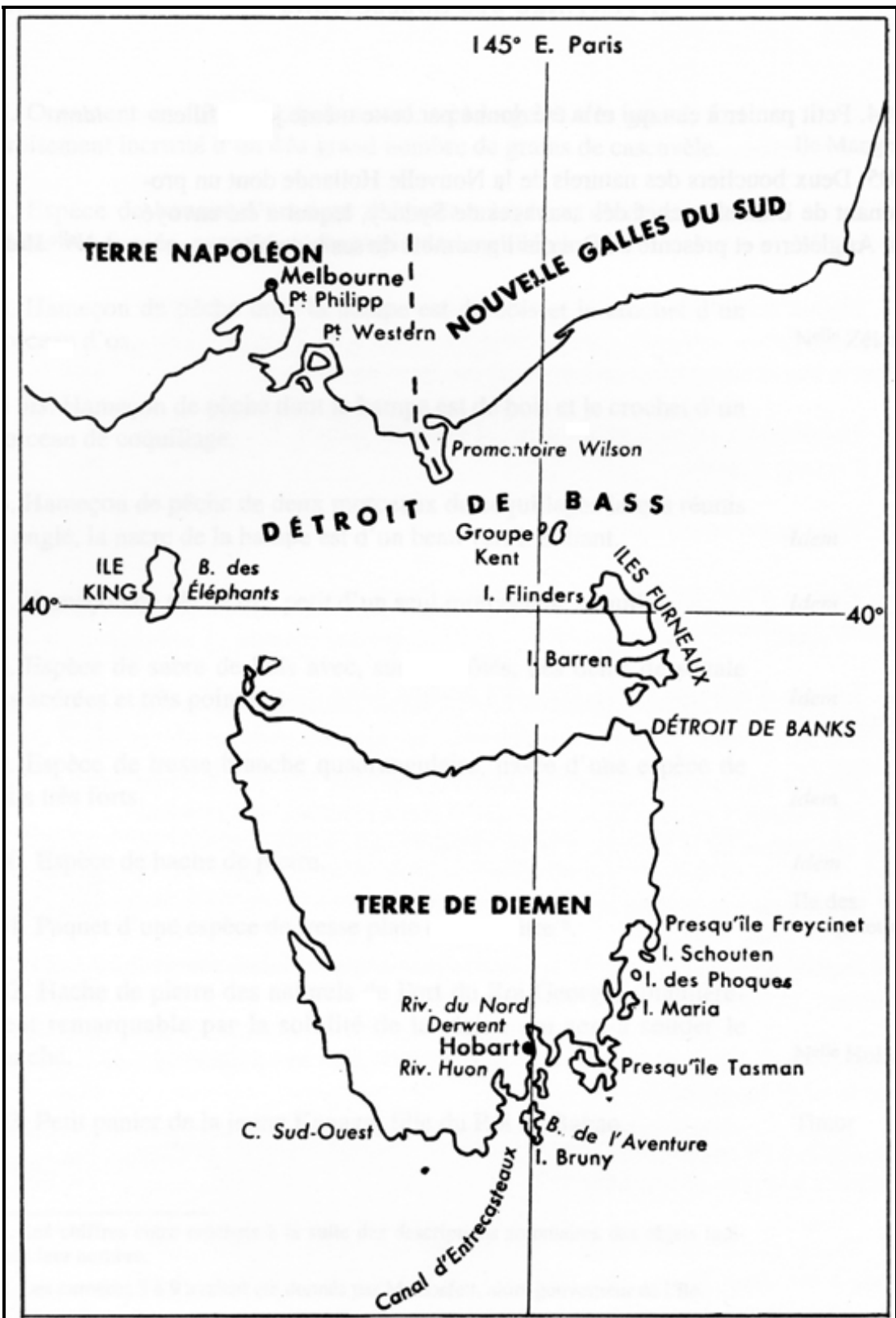

La Terre de Diémen et l'Île Maria en 1802, d'après l'Atlas historique, in Faivre 1953

La Terre de Diémen et I'Île Maria en 1802, d'après I'Atlas historique, in Faivre 1953 
Aux origines de l'anthropologie française. (1994)

Édition revue et corrigée par les auteurs, 1993.

Texte 10

\section{Des naturels que nous trouvions et de leur conduite envers nous (1804)}

\section{Par Nicolas Baudin}

Retour à la table des matières

Ce texte de Nicolas Baudin est inédit; il est extrait de la correspondance que Baudin entretint avec A.-L. Jussieu (alors directeur du Muséum d'Histoire naturelle) pendant l'expédition aux terres australes. Cette correspondance fut signalée par J.-P. Faivre et retrouvée par nous à la bibliothèque du Muséum national d'Histoire naturelle sous la cote: manuscrits, $n^{\circ}$ 2082. Elle est composée de huit lettres à Jussieu et d'une lettre adressée à Hamelin, capitaine du Naturaliste, et commandant en second de l'expédition. Ces lettres sont ainsi datées: 7 vendémiaire an IX (4 feuillets); 14 brumaire an IX (4 feuillets) : 4 floréal an IX ( 5 feuillets) - 13 vendémiaire an $X$ ( 1 feuillet) : 20 brumaire an XI (56 feuillets); 9 prairial an XI (4 feuillets); 23 thermidor an XI (I feuillet) ; 26 brumaire an XI (8 feuillets - lettre à Hamelin). Les passages présentés ici sont extraits de la longue lettre 
du 20 brumaire an XI, véritable rapport sur l'expédition. Cette correspondance de Baudin a une double importance : ethnologique certes nous y avons insisté dans notre présentation - mais aussi et surtout historique. Elle compte en effet parmi les rares documents dont nous disposons aujourd'hui sur les populations tasmaniennes qui ont été victimes d'un des plus méconnus mais des plus odieux génocides que les politiques et pratiques coloniales aient jamais commis. Découverts à la fin du XVIIIe siècle, leur territoire occupé et colonisé dès 1803 par les Anglais, ces peuples devaient ère exterminés jusqu'à leur dernier représentant en moins de soixante ans. Sur cette question, voir J. Bonwick, The Last of the Tasmanians, or the Black War of Van Dieme$n$ 's Land, Londres, Sampson Low, 1870 ; et A. Quatrefages, Hommes fossiles et hommes sauvages, Paris, Baillères, 1884, pp. 292-400 (réimpression Jean-Michel Place, Paris, 1988).

[170]

\section{Des naturels du Canal d'Entrecasteaux}

PENDANT NOTRE SÉJOUR à la grande Ance qui peut-être méritera un autre nom, nous eûmes de fréquentes entrevues avec les naturels qui pour ce moment se trouvaient sur l'île Bruny, car il n'y a pas de doute que les habitants de la terre de Diémen ne soient une peuplade errante n'ayant pour domicile passager que le lieu qui leur offre des moyens de subsistance ou plus facile ou plus abondante, mais je suis bien loin de la croire aussi bonne et aussi paisible qu'il en a plu au citoyen Labillardière 186 de nous la présenter dans la relation imparfaite

Jacques-Julien LABILLARDIERE (1755-1834), voyageur et naturaliste français. De 1791 à 1792, il fit partie de l'expédition commandée par d'Entrecasteaux et Huon de Kermadec, expédition dont le but était de retrouver les traces de Lapérouse. Labillardière en fit le récit dans: Relation du voyage à la recherche de Lapérouse, Paris, an VII, 2 vol. C'est à ce récit que Baudin s'en prend avec humeur, cherchant sans doute à régler un compte avec un « savant » qui avait sévèrement et partialement jugé les chefs de son expédition. Nul doute que la hargne et la critique de Baudin se trouvent ici exacerbées par ses propres rapports avec les savants de l'expédition dont il a le commandement, et dont on sait qu'ils ne furent pas des plus heureux. Baudin s'en 
et critique qu'il s'est permis de faire de l'expédition dans laquelle il n'était employé que comme botaniste.

Malgré que nous ayons comblé les habitants de l'île Bruny de présents, cela ne nous a pas garantis de plusieurs coups de sagaies, sans qu'aucune provocation de notre part y ait donné lieu. Heureusement les blessures qu'ils nous ont faites n'ont pas été dangereuses malgré la profondeur des plaies, ce qui me porte à croire que, [171] dans l'opi-

confiait déjà à Jussieu dans une lettre datée du 4 floréal an IX: «Pendant mon séjour à l'île de France, écrivait-il, quelques-uns des savants se sont occupés des productions du pays mais le plus grand nombre et surtout ceux qui, sous prétexte de maladie, se sont débarqués, sont constamment restés sur le port ou dans les habitations, occupés de leur "plaisir". Il est pénible pour moi d'avoir à m'en plaindre, mais comme je me suis fait une loi de dire la vérité telle qu'elle est, je vous dis franchement ce qui est arrivé et ce qui arrivera toujours quand ceux qu'on emploiera seront trop jeunes pour savoir apprécier la perte du temps. »

Dans la lettre du 20 Brumaire de I'an XI, Baudin devient plus incisif : " Mon opinion souvent opposée à la leur [aux savants de l'expédition] sur la manière de se conduire, d'examiner et de réfléchir, mes invitations réitérées pour qu'à l'exemple des citoyens Maugé [zoologiste, mort à I'île Maria en 1802] et Riedlé [chef jardinier, mort à Timor en 1801], qu'ils jugeaient bien au-dessous d'eux, ils fissent beaucoup d'ouvrage et peu d'esprit, sont cause qu'ils ont refusé depuis longtemps de me faire connaître les résultats de leurs travaux scientifiques. Peut-être ont-ils craint la censure d'un homme qui n'est pas savant à leur manière et qui plus d'une fois n'a pas approuvé des descriptions systématiques ou des conjectures hasardées. Mais ce qui me paraît le plus vraisemblable est qu'ils ont pensé que je pouvais m'approprier le mérite qu'ils accordent au moindre objet et que leur imagination féconde dénature en le voyant, comme quand ils le décrivent. Quoi qu'il en soit, je n'en désire pas moins qu'ils aient rempli l'attente du gouvernement et l'espoir des personnes véritablement instruites qui les ont jugés devoir être utiles dans cette campagne, et qu'il serait temps de terminer pour que la peur de la mort n'en conduise pas la plupart au tombeau avant l'époque fixée par la nature. Peu accoutumés aux privations habituelles à I'homme de mer, qui sait les supporter sans murmures, jusqu'à ce qu'une circonstance heureuse les lui fasse oublier: les conseils dictés par la raison suivis de l'exemple ont été méprisés par des savants et des officiers trop nouveaux pour avoir de l'expérience; en sorte que des consommations outrées dans le principe et dont tout l'équipage s'est ressenti par la suite, ont devancé le moment de la misère et produit les effets qui en sont inséparables. » 
nion du citoyen Labillardière, elles devraient être mortelles pour qu' on puisse dire qu'ils sont généralement traîtres et méchants ${ }^{187}$. En quittant la grande Ance, nous nous rendîmes, comme je l'ai déjà observé, au port nord-ouest; une partie des naturels que nous avions visités à notre première station vinrent se joindre à quelques autres que nous trouvâmes établis dans les environs de ce port. Tous nous ont paru ne faire qu' une même famille tant par la ressemblance de leur figure que par l'inconstance et la légèreté de leur caractère qu'il me paraît assez difficile de définir d'après ce qui m'est arrivé à moi-même.

À la première visite que nous fîmes à ceux du port nord-ouest le lendemain de notre arrivée, ils nous reçurent amicalement et sans armes, se firent peu prier pour s'approcher de nous, reçurent nos présents avec plaisir et enfin se prêtèrent de bonne grâce aux différentes attitudes que nous entendîmes prendre pour les dessiner 188 . Nous conversâmes plus de trois heures ensemble par signes ou autrement, assis sur l'herbe les uns parmi les autres et quiconque nous eût vus, nous aurait pris pour les meilleurs amis du monde. Néanmoins, leurs regards inquiets et leur conversation particulière me faisaient soupçonner quelques projets hostiles de leur part: j'annonçai que nous allions nous séparer en les invitant à se trouver le lendemain au même lieu. Ils comprirent bien ce que je cherchais à leur faire entendre et

Le citoyen Maurouard au moment où il s'embarquait pour retourner a bord fut atteint d'un coup de sagaie qui lui traversa la partie supérieure de l'épaule droite de part en part. La blessure fut large et profonde et ne se cicatrisa qu'au bout de dix jours. (Note de BAUDIN).

188 Dans le Voyage de découvertes aux Terres australes, F. Péron relate cet épisode d'une manière sensiblement différente: «Peu de temps après notre retour, le commandant lui-même revint d'une petite incursion qu'il était allé faire sur la grande terre avec le capitaine Hamelin, MM. Leschenault et Petit. Ces messieurs avaient de nouveau rencontré les naturels, et cette entrevue s'était encore terminée par une agression violente de leur part. En effet, M. Petit ayant dessiné plusieurs de ces sauvages, on se préparait à retourner au vaisseau, lorsqu'un d'entre eux s'élança sur le dessinateur pour lui arracher les dessins qu'il venait de faire: celui-ci voulant opposer quelque résistance, le sauvage, furieux, saisit une bûche, dont il allait assommer notre faible compagnon, si les autres ne fussent accourus à son secours. »F. PÉRON, Voyage de découvertes aux Terres australes, Paris, Imprimerie nationale, 1807, T. I, p. 237. 
promirent de s'y rendre. Nous nous dirigeâmes ensuite vers nos embarcations avec d'autant plus de confiance que nous avions acheté plusieurs de leurs sagaies; cachés derrière des arbres, ils nous suivirent à peu de distance des bords de mer, paraissant toujours gais et contents. Avant de nous quitter, je leur fis encore quelques présents et nous nous séparâmes, car ils ne voulurent pas nous accompagner jusqu'au bord du rivage malgré toutes nos insistances pour les y engager.

La distance à laquelle ils se trouvaient de nous paraissait suffisante pour n'avoir rien à craindre d'eux, néanmoins ils nous assaillirent d'une grêle de coups de pierres au moment de l'embarquement, dont heureusement personne ne fut atteint que moi et même assez vigoureusement, quoique lancées à la distance d'une portée de fusil ; comme je n'étais pas encore embarqué, je me retournai sur-le-champ en les mettant en joue. Ce seul mouvement les ayant aussitôt dispersés, ils se retirèrent dans les bois en jetant des cris très aigus que nous jugeâmes devoir être un signal de ralliement : [172] conjecture qui se vérifia bientôt car nous les vîmes en effet reparaître en bien plus grand nombre qu'ils n'étaient dans l'entrevue que nous venions d'avoir avec eux. Quoique tous fussent armés à leur manière, nous cherchâmes à les accoster de nouveau, mais ils se retirèrent dans les bois à mesure que nous avancions ; nous prîmes le parti de retourner à bord ${ }^{189}$.

189 Le ton légèrement ironique utilisé par Péron pour décrire et commenter l'incident de l'île Bruny semble traduire sa désapprobation à l'égard de l'attitude qu'eut Baudin : «Bien loin, cependant, écrit-il, de chercher à tirer vengeance d'une telle audace [celle du "sauvage" qui avait saisi une bûche pour la lancer sur Petit], on se plut à combler l'agresseur de nouveaux présents, dans l'espérance, sans doute, de calmer sa fureur par cette conduite généreuse, et de gagner sa bienveillance, ainsi que celle de ses compatriotes: mais à peine ces hommes farouches virent nos gens occupés à se rembarquer, qu'ils rentrèrent eux-mêmes dans le bois et un instant après il en sortit une grêle de pierres, dont une atteignit le commandant vers le bas du dos, et lui fit une large et forte contusion. Nos camarades, malgré cette perfidie, ne voulurent pas cesser d'être généreux. Vainement les sauvages se présentèrent à leurs coups, en les provoquant de dessus le rivage qu'eux-mêmes venaient de quitter, vainement ils brandissaient leurs sagaies, et multipliaient les gestes menaçants : pas un seul coup de fusil ne fut tiré contre eux. " Citant alors la relation de Leschenault, Péron continue ainsi : « Ces dernières hostilités furent commises de la 
On pourrait demander au citoyen Labillardière qui les a trouvés partout si bons et si paisibles, quels motifs ont pu les déterminer à attaquer de la sorte, et pour la seconde fois, ceux qui non seulement venaient de les combler de présents, mais encore qui, pour leur plaisir, s'étaient prêtés à leurs fantaisies, très désagréables pour ceux qui en furent l'objet car nous les laissâmes nous examiner de toute manière et satisfaire pleinement leur curiosité.

Je ne doute point que le citoyen Labillardière ne trouve dans les ressources de son imagination fertile une raison propre à accuser leur conduite dans ces deux circonstances, mais il ne lui serait peut-être pas aussi aisé de concevoir comment ces mêmes hommes qui devaient craindre les suites de notre ressentiment, se trouvaient le lendemain au rendez-vous désigné la veille, sans armes, suivis de leurs femmes et de leurs enfants, se livrant à nous à discrétion, quoique nous fussions en plus grand [173] nombre et mieux en état de défense. Pour moi,

part des naturels, sans que nous eussions donné lieu en aucune manière: au contraire, nous les avions comblés de présents et de bienfaits, et rien dans notre conduite n'avait pu les offenser. J'avoue que je suis surpris, après tant d'exemples de trahisons et de cruautés rapportés dans tous les voyages de découvertes, d'entendre répéter à des personnes sensées, que les hommes de la nature ne sont point méchants, que l'on peut se fier à eux ; qu'ils ne seront agresseurs qu'autant qu'ils seront excités par la vengeance, etc. Malheureusement beaucoup de voyageurs ont été les victimes de ces vains sophismes. Pour moi je pense, d'après tout ce que nous avons pu voir, qu'on ne saurait trop se méfier des hommes dont la civilisation n'a pas encore adouci le caractère, et qu'on ne doit aborder qu'avec prudence sur les rivages habités par de tels hommes ». PÉRON, op. cit., 1807, p. 237-238.

Bien que souvent dépeint comme un homme rigide et brutal, par Péron et par les savants de l'expédition, Baudin, si l'on en juge par la comparaison de ces deux récits et de leurs commentaires respectifs, ne se montre guère sous un tel jour ; il est loin d'être ce « rustre», ce «béotien» qu'ont volontiers décrit Péron et Leschenault. Les substantifs utilisés par l'un et par les autres pour désigner les autochtones («naturels» chez Baudin, « sauvages, hommes farouches » chez Péron et Leschenault); ceux employés pour définir et juger leur comportement (Baudin se garde de juger, alors que Péron et Leschenault parlent de «perfidie», de «trahison» de « cruauté ») ; la manière enfin d'envisager la conduite à tenir à leur égard, ne sont certes pas à l'avantage des savants, et notamment pas à celui de Péron qui, se piquant d'anthropologie, montre là une bien curieuse façon de la faire et de la concevoir, et, se voulant /'anthropologiste de l'expédition, une bien curieuse manière d'en être un. 
j'avoue tout bonnement qu'il m'est impossible de me faire une idée de leur caractère et j'attribue à un défaut de mémoire plutôt qu'à toute autre cause, leur conduite dans cette occasion.

Comme ce qui venait d'arriver ne me tranquillisait pas pour la suite du séjour que nous avions à faire dans ce port, je résolus de retourner à terre le lendemain, ne jugeant pas à propos de confier à d'autres, ni les moyens de défense que je comptais employer en cas de besoin, ni ceux que je voulais tenter pour rétablir entre les naturels et nous la bonne intelligence qu'il était nécessaire d'y voir régner. Le besoin de renouveler notre provision d'eau et de bois à brûler était une raison plus que suffisante pour ne rien négliger afin d'y parvenir. L'expérience, qui d'ailleurs $m$ 'avait appris que la supériorité en force n'est pas toujours I'unique ressource à employer pour se garantir des pièges de I'homme de la nature, fit que j'aimai mieux m'exposer avec prudence que d'avoir des inquiétudes continuelles sur le sort de ceux qui devaient être employés aux travaux qui nous restaient à faire.

Ayant débarqué dans le lieu désigné pour le rendez-vous, nous examinâmes les environs et n'y trouvâmes personne. J'en fus d'autant moins surpris qu'il ne me paraissait pas vraisemblable que les naturels osassent s'y montrer à découvert depuis leur conduite de la veille, aussi retournâmes-nous au bord de la mer pour y pêcher à la seine 190. Tandis que nous étions occupés à ce travail, on s'aperçut que trois d'entre eux étaient cachés derrière des arbres, d'où ils observaient attentivement tout ce que nous faisions. Je ne voulus point qu' on fit attention à eux et nous continuâmes à pêcher comme si nous avions ignoré qu'ils fussent près de nous, de sorte que nous avions déjà donné plusieurs coups de seine quand ils se décidèrent à se montrer de plus près et même à paraître sur le bord du rivage, ayant chacun une sagaie à la main.

Le capitaine Hamelin, suivi de deux hommes, sans arme apparente, fut alors au-devant d'eux en leur faisant des signes d'amitié et leur montrant les nouveaux présents que nous voulions leur faire. Ils se laissèrent approcher sans faire le moindre geste offensif, et bientôt ils furent tranquillisés sur nos intentions par les caresses qu' on leur

190 Filets disposés en nappe, et formant un demi-cercle. 
prodigua ou mieux encore par les présents qu'ils reçurent. La confiance même fut si promptement établie qu'ils ne firent aucune difficulté d'échanger leurs armes contre des boutons de métal et vinrent de leur propre mouvement au milieu de nous nous aider à tirer la seine. Ces hommes étaient cependant les mêmes que ceux qui, la veille, nous avaient assaillis de coups de pierres.

J'ignore si la manière de pêcher à la seine leur était connue avant cette époque, mais il paraîtrait que non, car, l'un des trois, et c'était le plus vieux, s'étant séparé des deux autres, revint peu de temps après suivi de dix-huit personnes au nombre desquelles se trouvaient trois femmes et huit enfants. Cette nouvelle compagnie ne tarda pas à se mêler avec nos gens, mais les enfants parurent dans le principe [174] timides et craintifs. Plusieurs même restèrent à l'écart malgré l'invitation de leurs parents, et ce ne fut que par les cris de joie et les marques de contentement qu'ils donnaient quand on retirait la seine, qu'ils se décidèrent à les venir joindre : ils devinrent par la suite aussi familiers qu'on pouvait le désirer.

Je ne m'étais pas attendu en descendant à terre à trouver autant de facilités à nous rapprocher les uns des autres, aussi je ne négligeai rien pour leur faire prendre de nous une idée avantageuse. Quelqu'un voulut leur faire des reproches sur ce qui s'était passé la veille, mais je lui imposai silence afin de les convaincre, si toutefois ils s'en rappelaient, que nous I'avions entièrement oublié.

Le contentement et la satisfaction qu'il avaient témoignés pendant le temps de la pêche me portaient à croire qu'ils recevraient avec plaisir une partie du poisson que nous avions pris et qui leur revenait de droit puisqu'ils nous avaient aidés: mais ils refusèrent constamment d'y toucher en nous faisant entendre qu'ils n'en mangeaient pas. Nos gens, en ayant préparé et fait bouillir devant eux pour leur dîner, leur en offrirent également sans pouvoir parvenir à leur en faire accepter. Ils ne firent pas plus de cas du biscuit ni du pain frais qu'ils ne recevaient que pour le jeter par terre après l'avoir considéré. Les enfants tinrent absolument la même conduite.

Parmi les présents que nous leur fîmes ce jour, les bouteilles parurent être celui qui leur fut le plus agréable. Aussitôt qu'on leur en 
donnait une, celui qui la recevait s'en allait bien vite la cacher comme s'il eût craint qu' on la lui ait redemandée.

La marmite qui avait servi à cuire le dîner de l'équipage fixa un moment leur attention. Comme elle pouvait leur être utile, j'en fis offre à celui qui me parut être le chef de famille; il l'examina de différentes manières et la jeta sur le sable comme une chose dont il faisait peu de cas.

Ces naturels ont une parfaite connaissance des armes à feu et paraissent en redouter les effets. Quand il nous arrivait de toucher aux nôtres, tous se levaient spontanément et la peur ou l'inquiétude se montraient dans tous leurs mouvements. Il n'était guère possible de les rassurer qu'en éloignant ceux qui les portaient. Pour nous prouver combien ils aimaient peu en entendre le bruit, ils nous engagèrent à ne point même tirer sur les oiseaux en nous faisant comprendre qu'ils nous les tueraient à coups de pierres, mais ils ne furent ni adroits ni heureux dans cette expérience, car ils ne touchèrent aucun de ceux qu'ils ajustèrent, quoiqu'à une petite distance!

Cette journée dont je ne m'étais pas fait une idée fort agréable devint, sans que j'en puisse dire la raison, une des plus amusantes que nous ayons passées dans le Canal, et depuis cette époque, nous n'eûmes que de légers mécontentements de leur conduite à notre égard. Les navigateurs qui par la suite seront dans le cas de communiquer avec eux, n'en doivent pas moins être sur leurs gardes. Trop de confiance comme trop de sévérité pourraient également les compromettre!

Les naturels de l'île Bruny, comme ceux de différentes autres parties du Canal, sont généralement d'une hauteur médiocre et assez mal faits. Presque tous ont les jambes épaisses, les pommettes des joues saillantes et le nez un peu écrasé, sans [175] cependant l'être autant que celui des Africains. Leur front large et avancé leur fait paraître les yeux petits au premier aspect, ce qui ne contribue pas à l'embellissement de leur figure.

Leur regard, sans être farouche, n'a rien d'agréable, quoiqu'ils l'aient vif et animé. La couleur de leur peau est d'un brun clair se rapprochant plus ou moins du noir, suivant qu'ils sont plus ou moins barbouillés de charbon pilé ou d'une terre rouge dont ils font usage pour 
plaire ou se rendre plus redoutables. Tous ont les dents bien rangées, très larges et blanches. Nous n'en avons point vu auxquels il en manque, mais plusieurs en avaient de cassées. Quelques-uns de nos savants ne manqueront pas de dire qu'ils leur ont trouvé les dents limées. C'est une erreur. Leurs cheveux sont laineux, peu épais, frisés et courts. Les élégants les enduisent de graisse ou de terre rouge, de manière qu'ils semblent porter perruque.

Le tatouage est un usage parmi eux, et je crois qu'aucun de nous ne conçoit comment ils $s^{\prime} y$ prennent pour que les marques qu'ils s'impriment sur le corps soient relevées en bosses très saillantes sur la superficie de la peau. Les endroits qui en sont les plus affectés sont la figure, le haut du bras, le derrière des épaules et le bas-ventre.

Leur principale nourriture ne paraît consister qu'en production marine, et particulièrement en homards, oreilles de mer, huîtres et autres coquillages. Les kangourous et les oiseaux en font aussi partie quand ils en peuvent attraper, mais ce n'est qu'accidentellement. Un de nos savants prétend qu'ils font usage d'une espèce de fougère dont la racine est farineuse. Il ajoute même avoir une preuve incontestable que dans les temps de disette, ils se nourrissent d'herbe. Comme il a la preuve de ce qu'il avance dans des excréments bien desséchés on pourra juger jusqu'à quel point cette conjecture est fondée!

Les femmes ne sont ni jolies ni bien faites, du moins celles que nous avons vues m'ont paru telles. Seules elles paraissent être chargées du soin du ménage et ont à pourvoir à la subsistance de toute la famille quant à ce qui concerne la pêche, exercice dans lequel elles ne manquent pas d'adresse. Nous les avons vues plus d'une fois revenir chargées à n'en pouvoir plus d'oreilles de mer ou de homards, qui n'avaient point ces énormes mordants dont parle le citoyen Labillardière quoique véritablement l'espèce existe puisque nous en avons vu l'échantillon.

Les dessins que je donne des huttes qui servent d'abris aux habitants de l'île de Diémen, ou de leurs ustensiles de ménage, feront mieux connaître combien ils sont misérables que tout ce que j'en pourrai dire.

Leurs pirogues, si toutefois on peut donner ce nom à trois faisceaux d'écorce d'arbre ou de tronc liés ensemble, et leurs armes sont 
les seules marques d'industrie que nous ayons remarquées. Les femmes semblent en avoir davantage à en juger par le travail des paniers dont elles se servent pour la pêche. Les enfants de l'un et l'autre sexe sont d'une gaieté aimable et fort enjouée. Quand la peur de nous se fut dissipée, ils devinrent si familiers qu'ils ne cherchaient qu'à jouer ou à s'amuser [176] avec nos gens. Tantôt ils les invitaient à la course, tantôt à cabrioler sur l'herbe ou à jeter des pierres. Toujours en mouvement, ils se plaisaient à faire quelques défis ou espiègleries à ceux auxquels ils donnaient la préférence ; tous en général ont la figure jolie, agréable et même spirituelle, ce qui me porte à croire que ce n'est que quand ils commencent à se défigurer par leur peinture grasse et malpropre qu'ils altèrent les traits du visage. Comme aucun, quoique déjà grand, n'était tatoué, il paraîtrait que cette cérémonie ne se fait qu'à une certaine époque. Ceux qui croient tout deviner assurent que ce doit être l'époque du mariage. Je suis bien loin de partager cette opinion, en ce que plusieurs m'ont paru d'un âge assez avancé pour en avoir exercé les fonctions.

Les habitants de l'île Bruny comme ceux de la grande île de Diémen sont au surplus des hommes primitifs de la nature, sinon dépourvus de toute intelligence, du moins au plus loin degré de civilisation qu'on puisse se les représenter. Nus de la tête aux pieds, il leur arrive parfois de se couvrir les épaules d'une peau de kangourou: usage qui paraît plus habituel aux femmes qu'aux hommes. Si la nature chez eux a des besoins, ils y satisfont au moment même sans s'éloigner avant ni après du lieu où ils se sont soulagés. Qu'ils soient en compagnie ou non, cela leur importe peu, aussi plusieurs fois nous ont-ils obligés de changer de place.

Un usage qui leur paraît particulier est de tenir presque constamment avec l'index et le pouce l'extrémité de leur prépuce, soit qu'ils marchent ou qu'ils restent au repos, ce qui fait qu'ils l'ont extrêmement long. Les femmes pour cacher leur nudité n'ont pas employé devant nous le même moyen dont parle le citoyen Labillardière. Celles qui avaient des enfants à la mamelle les portaient derrière le dos de différentes manières, soutenus par une peau de kangourou qui leur couvrait une partie du sein. 
Tous nos efforts, pendant notre séjour parmi les habitants de cette contrée n'ont pu leur faire comprendre l'usage et l'utilité de la plupart des présents que nous leur fîmes. Nos haches, nos couteaux, nos clous ne les ont jamais tentés. Après en avoir fait usage devant eux, on les leur donnait et un instant après ils nous les renvoyaient.

Plusieurs furent vêtus de casaques et de culottes longues, mais cet habillement était trop gênant pour eux pour qu'ils le conservassent longtemps. Aussitôt qu'ils étaient libres, ils se retiraient à l'écart et après s'en être débarrassés, ils en enlevaient les boutons et les laissaient au pied d'un arbre, ne se montrant que le lendemain dans leur parure ordinaire. Je pense que l'usage du fer leur est absolument inconnu, non seulement par le peu de cas qu'ils firent des outils que nous voulions leur donner, mais aussi par l'indifférence et le peu d'attention qu'ils apportaient à nous voir en faire usage. Semblables aux enfants que la moindre chose étonne, ils passaient successivement d'un objet à un autre, et celui qui avait le plus d'éclat obtenait toujours la préférence. Dans une visite que quelques-uns d'eux firent à ceux d'entre nous établis à l'observatoire, ils eurent l'occasion de voir deux chèvres et un mouton, ces animaux firent sur eux une grande impression: souvent ils leur [177] adressaient la parole, mais revenus de leur premier étonnement, ils ne s'en occupaient plus. La tente sous laquelle étaient déposés les instruments ne fut pas sans intérêt pour eux, et peut-être y seraient-ils restés plus longtemps qu' on ne l'aurait désiré, s'ils n'eussent trouvé le moyen d'y dérober une bouteille. La crainte d'être découverts, ou plutôt la peur de la rendre, les décida sans doute à se retirer, car après l'avoir passée à une de leurs femmes qui la cacha assez maladroitement dans sa peau de kangourou, ils partirent et ne reparurent plus.

Si peu de monde dans un espace aussi étendu porte à croire que la population de la grande île de Diémen n'est pas considérable. Il ne paraît cependant pas qu'ils aient de guerre entre eux, en ce que nous n'en vîmes aucun qui portât des blessures qui puissent nous le faire soupçonner: mais les plus âgés dont nous ayons eu connaissance $n$ 'avaient pas plus de quarante-cinq ou cinquante ans.

Nous n'avons rien rencontré à la terre de Diémen ni dans le Canal qui peut nous donner une idée de la manière dont ils disposent des 
morts. Comme on a cru remarquer qu'ils regardaient souvent le soleil, on s'est facilement persuadé qu'il devait être leur divinité : mais je n'ai rien vu de semblable et je les crois aussi dépourvus d'idées à ce sujet que d'industries pour leur propre conservation.

Je ne m'étendrai pas davantage sur ce qui est relatif aux naturels de la terre de Diémen, laissant à nos savants qui peut-être les ont mieux étudiés que moi, la relation complète des remarques particulières qu'ils ont dû faire et qui ne sont pas parvenues à me convaincre. [...]

\section{Des naturels de l'île Maria}

LES NATURELS que nous trouvâmes établis à l'île Maria nous semblèrent mieux faits et plus courageux que ceux du Détroit. Nos armes leur étaient connues et s'ils en redoutaient les effets, au moins ils aimaient à nous voir nous en servir. Plusieurs de ceux des nôtres qui en portaient furent invités à différentes reprises de leur tuer des oiseaux qu'ils jetaient dans le feu à mesure qu'on leur donnait. Les plumes en étaient à peine brûlées qu'ils les mangeaient aussitôt avec une voracité sans égale.

La famille qui habitait alors dans l'île n'était pas nombreuse. Nous ne vîmes jamais plus de vingt-deux personnes réunies, y compris les femmes et les enfants qui furent beaucoup plus sauvages et timides que ceux du Détroit. De tous nos présents, celui qui parut leur être le plus agréable fut des bouteilles. Leur façon de vivre, de se loger, de se couvrir, de s'armer, de se défigurer, de se tatouer et de parler, était la même que dans le Canal. On ne peut pas douter qu'ils n'aient la même origine. Leurs pirogues, quoique semblables par la forme et l'assemblage, ne différaient que par la matière, étant composées de trois faisceaux de roseaux au lieu de ceux d'écorce d'eucalyptus que nous leur connaissions avant que d'avoir vu celles-ci.

[178]

Pendant cette relâche, nous n'eûmes aucun sujet de mécontentement de la part des naturels. Dès notre première entrevue à laquelle 
j'étais présent, ils nous reçurent amicalement quoique armés de leurs sagaies. Comme ils étaient accompagnés de leurs femmes et de leurs enfants, c'était pour moi une preuve évidente qu'ils n'avaient aucun projet hostile, du moins pour le moment. Leur contenance ferme et assurée à mesure que nous les approchâmes, nous prouva qu'ils n'avaient nullement peur de nous, malgré que nous fussions en plus grand nombre.

Une aventure assez particulière qui arriva à un des charpentiers, du Naturaliste, mérite cependant d'être citée. Occupé ainsi que trois autres à couper du bois à brûler, plusieurs naturels armés à leur manière $s$ 'avancèrent vers eux. Les suites de cette rencontre prouvent qu'ils n'avaient pas de mauvaises intentions, mais la peur s'étant emparée de nos gens, ces derniers ne songèrent qu'à la fuite au lieu de se réunir pour en imposer en cas de besoin. Par cette conduite peu réfléchie, un d'eux se trouva entouré par des naturels auxquels il se livra à discrétion, n'ayant pour toute défense que sa hache. Ceux-ci s'en étant rendus maîtres le conduisirent immédiatement à l'eau et l'ayant mis nu de la tête aux pieds, firent une inspection scrupuleuse de tout son corps: après quoi, ils le laissèrent tranquillement se retirer et s'en aller, sans lui faire aucun mal. Soit que sa hache leur ait paru une chose inutile ou non, ils en firent si peu de cas qu'ils la lui rendirent.

Il serait peut-être aussi intéressant pour l'histoire de l'homme de savoir ce que pensait le charpentier dans la circonstance où il se trouvait que de connaître le motif qui excita la curiosité des naturels. 
Aux origines de l'anthropologie française. (1994) Édition revue et corrigée par les auteurs, 1993.

Texte 11

\section{Expériences sur la force physique des peuples sauvages (1807)}

\section{Par François Péron}

Retour à la table des matières

Le texte qui suit est I'un des mémoires de Péron, placé en annexe du Voyage aux Terres australes, Paris, Imprimerie nationale, 1807, pp. 446-483.

C'est à l'instigation de Buffon et de Guéneau de Montbelliard 191 que, dès le début des années 1780 , I'ingénieur en mécanique Edmé Régnier (1751-1825) s'exerça à fabriquer un instrument qui répondît aux exigences des deux naturalistes: se proposant de mesurer d'une manière comparable les forces relatives de l'homme dans ses différents âges ainsi que dans ses différents états de santé, Buffon et Guéneau voulaient utiliser une machine précise, portative, d'un maniement simple - donnant des appréciations directes et rendant les effets compa-

191 Une première version de ce texte a paru dans Gradhiva n ${ }^{\circ} 1,1986, \mathrm{pp}, 17-21$. 
rables -, avec laquelle pût être mesurée la force musculaire qui agit non seulement sur un doigt ou sur une main mais sur chaque membre pris séparément et sur toutes les parties du corps. Les appareils de Graham et de Leroy inventés quelques années plus tôt, et connus des deux savants, ne les satisfaisaient pas : trop volumineux ou trop spécifiques, ils manquaient de souplesse d'utilisation et, surtout, ne permettaient pas d'effectuer des mesures comparatives 192.

[180]

La mort de Guéneau en I785, celle de Buffon en 1788, puis la Révolution française (pendant laquelle Régnier devint inspecteur de la Manufacture des armes portatives créée par le Comité de Salut public) interrompirent les recherches et essais de l'ingénieur. Ce n'est qu'en 1796 qu'il les reprit, grâce aux recommandations du physicien CharlesAugustin Coulomb qui, dans un mémoire lu à l'Institut la même année, proposait de "déterminer la quantité d'action journalière que les hommes (Français) peuvent fournir par leur travail particulier, suivant les différentes manières dont ils emploient leur force », en vue de développer l'industrie et, ce faisant, de redresser l'économie nationale 193. Mis au point à la fin de 1796, I'instrument rentrait dans la catégorie des dynamomètres, c'est-à-dire des appareils qui permettent de mesurer la force au moyen de l'élasticité des corps solides.

Dans sa forme originale, qui ne variera guère, le dynamomètre de Régnier se compose d'un ressort en acier corroyé et trempé, long de 32 centimètres, ployé en ellipse (soumis à une épreuve plus forte que ne porte sa graduation af in qu'il ne puisse perdre de son élasticité par l'usage), et d'une plaque en cuivre de forme semi-circulaire dont le centre est fixé au milieu de la branche supérieure du ressort par un

192 Cf. BUFFON, De L'homme, Paris, Maspéro, 1971 (édition de Michèle Duchet), p. 130 sq. ; ED. RÉGNIER, « Description et usage du dynamomètre », Journal de I'École polytechnique, 5e cahier, 1796, pp. 160-172.

193 C.-A. COULOMB, «Expériences destinées à déterminer la quantité d'actions journalières », Comptes rendus des Mémoires de l'institut, $n^{\circ} 6,1798$, pp. 1825 : ANONYME, « Sur le dynamomètre de Régnier, Journal des Mines, T. 17, $n^{\circ}$ 97, 1804, pp. 51-76 (cet article contient le compte rendu des expériences faites au Comité central de l'Artillerie le 3 germinal de l'an 4 par F.-M. Aboville, Dulauloy, d'Hennezel et Lariboisière, pp. 69-76). 
support d'acier à patte et à vis. Sur la branche inférieure du ressort, en un point diamétralement opposé, est également vissé un autre support d'acier destiné à recevoir un petit repoussoir en cuivre qui, faisant levier, agit sur une aiguille fixée à son axe par une vis au centre du cadran. Sous la patte de l'aiguille est collée une petite rondelle de peau, afin de déterminer sur le cadran un mouvement doux et uniforme dont l'effet est de maintenir l'aiguille à la place où elle a été amenée par le petit repoussoir faisant levier. Cette aiguille se termine par un double index qui balaye deux arcs gravés sur la plaque en cuivre. L'arc supérieur est divisé en myriagrammes, l'arc inférieur en kilogrammes: l'étalonnage a été réalisé au moyen de poids. Le dynamomètre de Régnier peut être utilisé soit par pression, soit par traction.

Dans le premier cas, pression, il permet de mesurer la force des mains qui, placées au plus près du centre du dispositif tenu à bout de bras de manière à former un angle de quarante-cinq degrés avec l'axe du corps (position optimale), serrent les deux branches du ressort : la force exercée doit être lue sur l'arc inférieur qui donne la valeur en kilogrammes. Chaque degré de l'échelle de pression vaut 500 grammes.

Dans le deuxième cas, traction, il permet de mesurer la force des reins (ou, à l'aide de mousquetons engagés aux extrémités du ressort, toute autre force de traction comme celle des animaux de trait par exemple). L'appareil est alors disposé verticalement. Un oeil du ressort est accroché à un cran de la crémaillère sur [181] l'empattement de laquelle le sujet pose les pieds, le corps d'aplomb; I'autre oeil du ressort est fixé au crochet d'une poignée double au moyen de laquelle le sujet tire bras tendus. La force développée doit être lue sur I'arc supérieur qui donne la valeur en myriagrammes. Chaque degré de l'échelle de tirage vaut un myriagramme, soit dix mille grammes.

D'un maniement simple, d'une lecture aisée et d'un transport facile (l'appareil ne pèse guère plus d'un kilogramme), le dynamomètre de Régnier eut un certain succès. En plus de son utilisation par François Péron de 1800 à 1804 pour mesurer la force des «sauvages» de Timor, de Tasmanie et d'Australie (cf. infra), le dynamomètre de Régnier se prêta à des utilisations plus prosaïques, techniques et militaires comme la mesure de la résistance des différents types de charrues, de chariots ou de voitures, comme celle aussi des affûts de ca- 
nons (mesure qui eut son importance si l'on se rappelle que Bonaparte avait fondé sa stratégie sur la mobilité de l'artillerie), comme celle encore de la force de recul d'un canon (qui devait déterminer la puissance de la poudre) ou de la force musculaire des conscrits, laquelle évaluée pouvait décider de leur arme d'incorporation... 194

Le modèle reproduit en frontispice de cet ouvrage provient du Musée de l'Armée à Paris. C'est là que, après des recherches d'abord infructueuses, nous l'avons retrouvé grâce à la persévérance du lieutenant-colonel Neuville, conservateur et spécialiste des armes du XIXe siècle, qui le découvrit en février 1983 au fond d'une réserve dudit Musée. Bien que la fiche d'inventaire ne comportât aucune indication sur son origine exacte, tout porte à croire qu'il s'agit d'un modèle analogue au prototype de 1796. Le mécanisme et la géométrie de l'appareil - qui connut quelques améliorations et transformations en 1804195 - sont en tous points identiques à la description et aux schémas que Régnier a publiés en 1796 196. Par ailleurs, Régnier fut le fondateur et le premier conservateur du Musée de l'Artillerie en 1796, qui est à l'origine directe du Musée de l'Armée; le poinçon du Musée de l'Artillerie est du reste apposé au centre du cadran. Ce qui laisse à penser que ce dynamomètre y fut déposé par lui ou par l'un de ses tout premiers utilisateurs entre 1796 et 1804.

C'est probablement un instrument de ce type - si ce n'est cet appareil lui-même serré dans une mallette de voyage spécialement conçue pour l'y loger avec tous ses accessoires - que François Péron emporta le 19 octobre 1800 sur la corvette Le Géographe, armée par l'Institut de France et placée sous le commandement du capitaine Baudin, et avec lequel il allait effectuer les premières mesures anthropométriques de l'anthropologie française pendant les quatre années que devait durer son voyage aux Terres australes. En 1817, Edmé Régnier rendait ainsi hommage à [182] «/'anthropologiste » François Péron: «M. Pé-

194 Cf. ED. RÉGNIER, « Mémoire sur le dynamomètre», Bulletin de la Société $d^{\prime}$ 'encouragement pour l'industrie nationale, $n^{\circ} 156,1817$, pp. 133-147.

195 Cf. ANONYME, art. cit., 1804, pl. 1.

196 Cf. ED. RÉGNIER, art. cit., 1796. 
ron, naturaliste d'un mérite distingué, a été porté d'observer que les peuples sauvages étaient moins forts que les hommes civilisés, et il a démontré, d'une manière évidente, que le perfectionnement de l'ordre social ne détruit pas nos forces physiques, comme l'ont prétendu quelques personnes (...). Quoique les sauvages soient ordinairement moins forts que les hommes civilisés, néanmoins ils attachent un grand prix à la force et ne peuvent pas, sans une espèce d'indignation, se voir vaincus, surtout par des hommes qui ne font pas partie de leurs peuplades. Voici comment M. Péron a été conduit à faire cette remarque intéressante (Régnier cite un passage du Tome 1 du Voyage aux Terres australes, rédigé par François Péron en 1807): "[22 février 1802] Fatigué de tous les mauvais procédés des sauvages de l'île Maria, je résolus de terminer enfin cette périlleuse entrevue; mais, voulant à tout prix répéter quelques observations que j'avais commencées déjà dans le Canal [d'Entrecastaux], sur le développement de la force physique des peuples de ces contrées, je fis apporter le dynamomètre de Régnier, du canot où je l'avais laissé jusqu'alors :j'espérais que la forme de l'instrument et son usage pourraient peut-être fixer l'attention des hommes farouches que je voulais soumettre à son épreuve. Je ne me trompais pas: ils admirèrent l'instrument; tous voulaient le toucher en même temps, et j'eus beaucoup de peine à empêcher qu'il ne fût brisé. Après leur en avoir fait concevoir l'objet par un grand nombre d'essais que nous fimes dans cette vue, nous commençâmes à les faire agir eux-mêmes sur l'instrument; et déjà sept individus s'y étaient soumis, lorsqu'un de ceux qui s'étaient essayés d'abord, et qui n'avaient pas pu faire Marcher l'aiguille du dynamomètre aussi loin que moi, parut indigné de cette impuissance; et, comme pour donner à I'instrument un démenti positif il s'approcha, me saisit le poignet avec colère, et semblait me défier de me dégager. J'y parvins cependant après quelques efforts: mais l'ayant à mon tour saisi de toute ma force, il lui fut impossible, quoi qu'il pût faire, de se délivrer ; ce qui parut le couvrir de confusion et le remplir de colère." „ 197

Plus tard, en 1839, Joseph-Marie de Gérando parlera des «belles expériences dynamométriques de Péron qui ont prouvé que la civilisa-

197 Cf. ED. RÉGNIER, art. cit., 1817, pp. 142-143. 
tion donne même un plus haut degré d'énergie aux forces musculaires de l'homme » 198.

Il faudra cependant attendre 1884 pour que quelques doutes soient avancés sur la valeur des expériences de Péron. Reproduisant les mesures obtenues par celui-ci, $A$. de Quatrefages les commente ainsi : «Ces chiffres justifient ce que dit Péron de la vie sauvage. Bien souvent, par ses conditions mêmes d'existence, elle tend à affaiblir les organismes. Toutefois il ne faudrait pas trop généraliser cette conclusion. On sait en particulier combien les Polynésiens l'emportaient sur les Blancs. Dans les expériences de Péron, les Européens familiarisés avec l'emploi des instruments [183] et dirigeant leurs efforts avec intelligence, ont dû se servir du dynamomètre bien mieux que des sauvages. » 199

Quoi qu'il en soit, les mesures dynamométriques de Péron, présentées en leur temps comme rigoureuses, reprises et de fait légitimées pendant près d'un demi-siècle par des philosophes et des naturalistes, pouvaient justifier toute mission civilisatrice, partant toute entreprise colonialiste... L'« état sauvage » n'était plus idyllique, mais il n'était pas irrémédiable. Bien plus, il n'était pas «primitif »puisque Péron pensait que la civilisation peut réduire ces siècles qui, selon Gérando, séparaient le sauvage du civilisé. Le paradis de l'homme naturel était définitivement perdu, mais celui de l'homme civilisé devait le lui faire retrouver. La faiblesse du sauvage venait par contraste souligner la force non seulement morale mais physique du civilisé, rassurer ce dernier dans son corps et l'assurer de son bon droit en lui révélant que le progrès était d'abord une question de force et d'énergie ; en l'occurrence le droit de faire partager au Sauvage les bienfaits de la civilisation laquelle, pour Péron, était plus que jamais un idéal, un paradis à exporter... une source d'énergie.

198 Cf. J.-M. DE GÉRANDO, De la bienfaisance publique, Paris, Renouard, 1839, p. 276.

199 A. DE QUATREFAGES, Hommes fossiles, hommes sauvages, Paris, Baillière \& Fils, 1894, p. 325 (réimpression Jean-Michel Place, Paris, 1986). 
Cela reviendrait-il à trop forcer les mots et la chose que de voir dans le dynamomètre de Régnier - appareil qui, dans sa position de tirage, se présente comme une sorte de ready-made qu'affectionnait Marcel Duchamp ou comme l'un de ces objets détournés que n'eussent pas renié les surréalistes - un instrument au sens strict du colonialisme?

ELLE EST RÉCENTE encore, cette époque singulière, où l'on vit des hommes célèbres, entraînés par une imagination ardente, aigris par les malheurs inséparables de notre état social, s'élever contre lui, en méconnaître les bienfaits, et réserver pour l'homme sauvage toutes les sources du bonheur, tous les principes de la vertu. Leur funeste éloquence égara l'opinion; et pour la première fois on vit des hommes sensés gémir sur les progrès de la civilisation, et soupirer après cet état misérable, illustré de nos jours sous le nom séducteur d'état de nature... Heureusement les voyageurs modernes, en nous faisant successivement connaître tant de peuples sauvages, nous ont permis d'apprécier ces vains sophismes à leur juste valeur; et notre expédition, sous ce rapport, aura pu servir la vraie philosophie.

De tous les biens dont les apologistes de l'homme sauvage se complurent à le gratifier, la force physique est celui sur lequel ils insistèrent plus particulièrement et plus constamment. Produit et compagne d'une santé vigoureuse, la force physique serait, en effet, l'un des premiers titres à la supériorité ; et si véritablement elle 


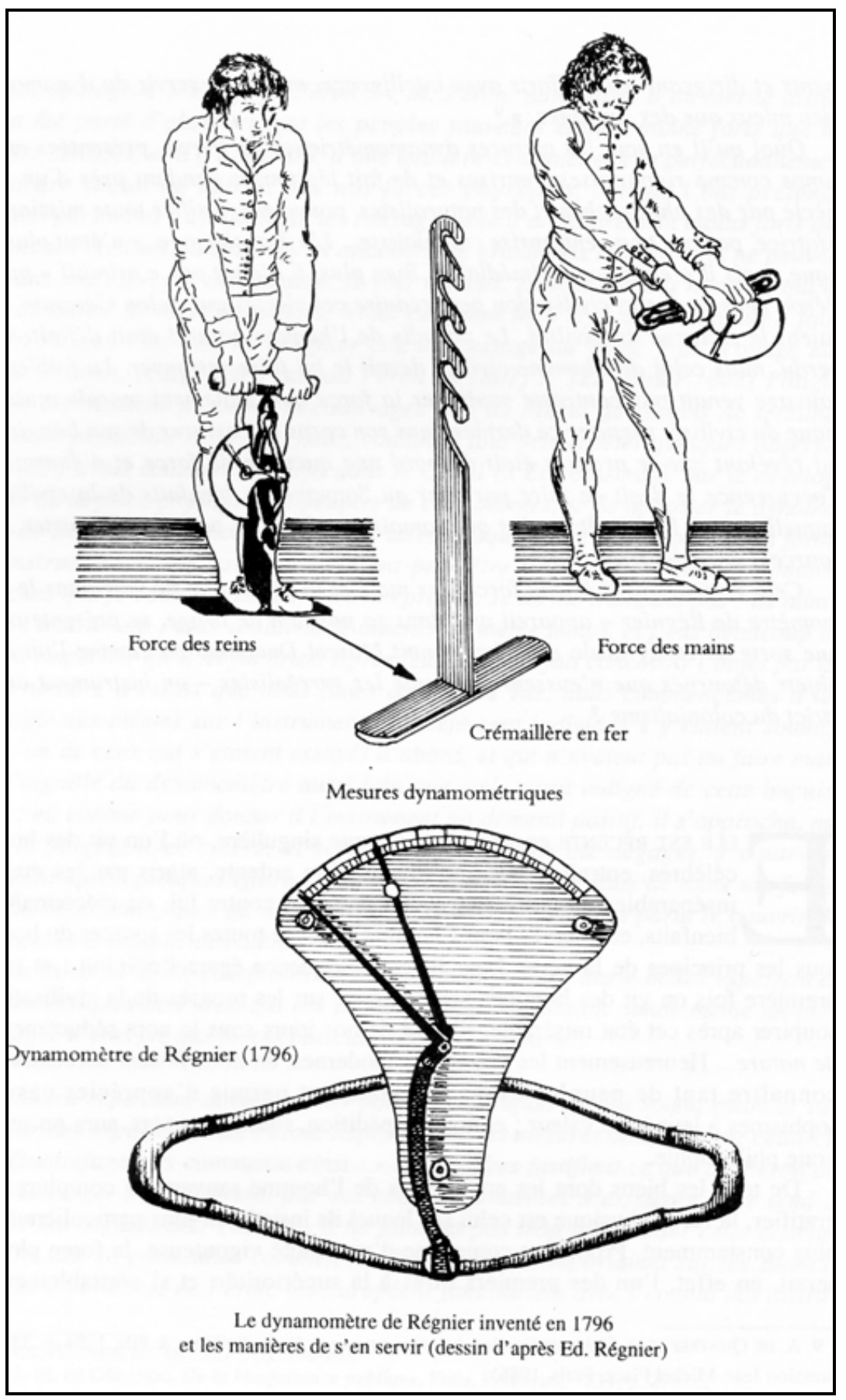

Le dynamomètre de Régnier inventé en 1796 et les manières de s'en servir (dessin d'après Ed. Régnier) 
devait être l'apanage exclusif ou même plus particulier de l'état sauvage, la civilisation, il faut en convenir, nous aurait ravi l'un des gages les plus certains du bonheur. Aussi les détracteurs de l'ordre social ont-ils rapporté leurs déclamations les plus éloquentes à cette espèce de dégradation de l'homme civilisé, et se sont-ils efforcés de la consacrer en principe. Jusqu'à ce jour, cependant, on a manqué de tout moyen pour comparer exactement la force des individus et des peuples: jusqu'à ce jour on n'a fait aucune expérience directe sur cet objet. M. Coulomb, dans le beau mémoire qu'il soumit en l'an VI à I'Institut, n'avait d'autre objet que de déterminer la quantité d'action journalière que les hommes (Français) peuvent fournir par leur travail particulier, suivant les différentes manières dont ils emploient leurs forces. Commandé par les circonstances, cet illustre physicien n'avait pu qu'indiquer les expériences à faire sur les différents peuples, et les modifications diverses que la force devrait présenter suivant les climats et la nature des aliments. Nous aurons bientôt l'occasion de justifier, par nos propres expériences, toute la justesse de ses aperçus à cet égard. Cette partie de l'histoire de l'homme était donc, au moment de notre départ d'Europe, l'une de celles qui réclamaient davantage l'intérêt des naturalistes et leur zèle.

L'invention récente du dynamomètre de Régnier venait d'ouvrir une nouvelle carrière en ce genre. Sans être parfait, sans doute, sans pouvoir fournir le terme précis de la force absolue, cet instrument donne du moins des approximations directes : il rend les effets comparables: et par les nombreuses expériences que j'ai pu faire pendant trois ans, j'ai acquis la certitude qu'il était, à cet égard, beaucoup plus rigoureux qu'on ne pourrait le soupçonner d'abord. J'avais l'avantage de le porter le premier au-delà des mers, et de pouvoir l'employer au milieu des peuples de l'hémisphère austral : je n'ai rien négligé pour en tirer tout le parti possible; et si les circonstances ne m'ont pas permis de donner à mes travaux en ce genre tout le développement dont ils étaient susceptibles, du moins me suis-je efforcé constamment de leur assurer un avantage précieux, celui de l'exactitude. 


\section{Section I \\ Expériences sur les Peuples sauvages \\ de la terre de Diémen}

SUR LA TERRE de Diémen, sur l'île Maria qui l'avoisine, il existe une race d'hommes tout à fait différente de celle qui peuple le continent de la Nouvelle-Hollande. Pour la taille, les individus se rapprochent assez des Européens : mais ils en différent par leur conformation singulière. Avec une tête volumineuse, remarquable surtout par la longueur de celui de ses diamètres qui, du menton se dirige vers le sinciput, avec des épaules larges et bien développées, des reins bien dessinés, des fesses généralement volumineuses, presque tous les individus présentent [186] en même temps des extrémités faibles, allongées, peu musculeuses, avec un ventre gros, saillant et comme ballonné. Du reste, sans chefs proprement dits, sans lois, sans aucune forme de gouvernement régulière, sans arts d'aucune espèce, sans aucune idée de l'agriculture, de l'usage des métaux, de l'asservissement des animaux: sans vêtements, sans habitation fixe, sans aucune retraite qu'un misérable abat-vent d'écorce pour se défendre de la froideur des vents du sud, sans autres armes que le casse-tête et la sagaie: toujours errant au milieu des forêts ou sur le rivage des mers, I'habitant de ces régions réunit sans doute tous les caractères de l'homme non social ; il est par excellence /'enfant de la nature. Combien il diffère cependant, soit au moral, soit au physique, de ces tableaux séduisants que l'imagination et l'enthousiasme créèrent pour lui, et que l'esprit de système voulut ensuite opposer à notre état social! ...

Avec des hommes aussi farouches, nos rapports ont été, sinon rares, au moins difficiles et périlleux, et la plupart de nos entrevues [...] se sont terminées par des agressions hostiles de leur part.

Si nos relations générales étaient si délicates, les observations dynamométriques l'étaient bien davantage encore. Aussi, malgré mon zèle, n'ai-je obtenu sur ces rivages que des données peu nombreuses et même incomplètes, puisqu'il ne m'a été possible d'engager aucun naturel à essayer sa force rénale. Néanmoins toutes mes observations 
ayant été faites sur des individus des mieux constitués de la nation, et les résultats en ayant été bien décidés, et bien constants surtout, on peut, sans crainte d'erreur, les appliquer à la généralité des individus de cette première race : or, ces résultats indiquent tous un défaut de vigueur véritablement extraordinaire. En effet, bien que mes expériences aient été répétées sur la classe la plus vigoureuse de la population, celle de 18 à 40 ans, aucun Diéménois n'a pu faire marcher l'aiguille de pression au-delà du 60e degré ; et le terme moyen des douze observations que j'ai pu faire, n'est que de 50, 6 kilogrammes. Nous verrons bientôt combien sont grandes les différences des épreuves faites sur les Européens. L'opposition des forces d'homme à homme vient confirmer ces premières données de l'instrument. Nos matelots eurent constamment l'avantage lorsqu'ils luttèrent contre les sauvages, et ces dernier ne furent pas plus heureux avec l'un de nos officiers, M. Maurouard : celui d'entre eux qui nous avait paru le plus robuste, après avoir joué quelques instants avec lui, voulut le provoquer à la lutte: l'officier français le terrassa. Contraint moi-même, sur l'île Maria, de subir, pour la force du poignet, une épreuve analogue, j'en sortis avec un succès égal [...].

De l'ensemble de ces faits et de ces expériences, on peut induire que le terme moyen de la force des peuples sauvages de la terre de Diémen et des îles qui l'avoisinent, est de beaucoup inférieur à celui que, par les mêmes moyens et dans les mêmes lieux, nous avons observé à l'égard des Européens.

[187]

Section II

Expériences sur les Peuples sauvages

de la Nouvelle-Hollande

TOUTE LA NOUVELLE-HOLLANDE, depuis le promontoire de Wilson au sud jusqu'au cap d'York au nord, paraît être habitée par une seconde race d'hommes essentiellement différente de celles qu'on a connues jusqu'à ce jour. La stature de ces hommes est à peu près la même que celle des habitants de la terre de Diémen, mais, indépen- 
damment de plusieurs autres caractères qu'il n'est pas de mon objet de retracer maintenant, ils différent surtout de ces derniers par la couleur moins foncée de la peau, par la nature de leurs cheveux lisses et longs, et par la configuration remarquable de leur tête, qui, moins volumineuse, se trouve déprimée en quelque sorte vers son sommet, tandis que celle des Diéménois est, au contraire, allongée dans le même sens. Le torse des individus de ce nouveau peuple est aussi généralement moins développé : du reste, même disproportion entre les membres et le tronc, même faiblesse, même gracilité de membres, et souvent aussi même tuméfaction du ventre. Pour ce qui concerne l'état social, les habitants de la Nouvelle-Hollande sont, à la vérité, tout à fait étrangers encore à la culture des terres, à l'usage des métaux: ils sont, comme les peuples de la terre de Diémen, sans vêtements, sans arts proprement dits, sans lois, sans cultes apparents, sans aucun moyen assuré d'existence: contraints, comme eux, d'aller chercher leur nourriture au sein des forêts ou sur les rivages de l'Océan. Mais déjà les premiers éléments de l'organisation sociale se manifestent parmi eux: les hordes particulières sont composées d'un plus grand nombre d'individus; elles ont des chefs; les habitations, quoique bien grossières encore, sont plus multipliées, mieux construites; les armes sont plus variées et plus redoutables; la navigation est plus hardie, les canots sont mieux travaillés, les chasses plus régulières, les guerres plus générales: le droit des gens n'y est déjà plus étranger : enfin, ces peuples ont assujetti le chien; il est le compagnon de leurs chasses, de leurs courses et de leurs guerres. Du reste, aussi farouches que les Diéménois, ils se montrent encore plus intraitables envers les étrangers. Dampier, Cook, La Pérouse et Flinders ont été forcés en différents lieux, à diverses époques, de faire usage des armes à feu pour repousser leurs insultes, et nous-mêmes nous n'avons évité cette cruelle nécessité, qu'en nous condamnant à la retraite.

Dix-sept sauvages de ce continent austral se sont soumis à l'expérience du dynamomètre. [...] Parmi les individus, quatorze sont de l'âge de 18 à 40 ans, et conséquemment appartiennent aux époques de la vie où I'homme est dans toute sa vigueur. Néanmoins ces sauvages se présentent ici avec le même caractère de faiblesse que nous venons d'observer dans ceux de la terre de Diémen. Un seul a pu faire marcher l'aiguille de pression jusqu'au 62e degré, et le terme moyen de la for- 
ce de ces quatorze individus excède à peine $51^{\circ}$; quelques-uns même ont paru [188] tellement faibles, qu'ils n'ont pu outrepasser $40^{\circ}$.

Pour la force des reins, mêmes résultats: si l'on excepte le jeune Ourou-Maré, qui a fait marcher l'aiguille jusqu'à $19^{\circ}$, aucun n'a pu atteindre jusqu'à $18^{\circ}$; et le plus grand nombre est tellement resté audessous de ce dernier point, que le terme moyen de la force des quatorze individus dont je viens de parler, est à peine de 14,8. Nous pouvons donc déduire, de cette seconde série d'observations, une conséquence analogue à celle que nous a fournie la première, c'est-à-dire que la force physique acquiert chez les sauvages de cette partie de la Nouvelle-Hollande un développement peu considérable. Nous aurons bientôt l'occasion de présenter quelques aperçus sur les causes qui paraissent plus particulièrement déterminer cette faiblesse.

\section{Section III \\ Expériences sur la Force physique des Habitants de Timor}

EN PASSANT de la terre de Diémen à la Nouvelle-Hollande, nous nous sommes, pour ainsi dire, élevés d'une manière insensible du dernier degré de l'état sauvage à celui qui le précède immédiatement. Pour conserver cette précieuse gradation de l'état social, il faudrait pouvoir parler maintenant des peuples de la Nouvelle-Guinée, supérieurs à ceux de la Nouvelle-Hollande : redescendant ensuite vers la Nouvelle-Zélande, nous y trouverions, d'après les relations les plus authentiques, le quatrième degré de l'organisation sociale les peuplades du grand Océan équatorial nous en offriraient le cinquième échelon Timor et les Moluques m'ont paru n'en présenter que le sixième. Malheureusement les trois degrés intermédiaires nous manquent encore: et je me garderai bien, à défaut d'observations positives, d'avoir recours à des analogies, toujours plus ou moins insuffisantes, et souvent trompeuses. Je me contenterai d'exposer les résultats de mes propres expériences sur les peuples malais de Timor et des îles voisines, Rotti [Pülü Rotte], Soumbawa [Siimbawa], Simaôô [Semawii], Macassar, Ende et Solor. Appartenant à la même race, habitant des mêmes ré- 
gions, soumis aux mêmes lois, aux mêmes usages, se nourrissant des mêmes aliments, tous m'ont semblé pouvoir être, sans erreur sensible, confondus dans les mêmes tableaux. Il en est de même de quelques Chinois qui, depuis plusieurs siècles, fixés de père en fils sur ces rivages, peuvent en être regardés comme indigènes. Au surplus, pour prévenir toute objection à cet égard, j'ai soigneusement indiqué la patrie de chacun des individus soumis à mes observations.

Les Malais, ainsi que je l'ai dit ailleurs, sont étrangers aux îles du grand archipel d'Asie. Conquérants farouches et sanguinaires, ils les ont envahies à une époque dont I'histoire et même la tradition ne gardent aucun souvenir. Établis sur le rivage de la mer, occupant toutes les côtes, ils sont réunis en sociétés nombreuses et régulières: [189] ils habitent dans des villes ou des villages plus ou moins étendus. Soumis à des rois plus ou moins puissants, parvenus, par leur commerce habituel avec les Européens, à un état de civilisation assez avancé, ils exercent différents arts, ils se livrent à la culture des terres, au soin des troupeaux, à la pêche, au commerce, à la navigation, autant que leur apathie naturelle le permet et que leurs besoins l'exigent. Issus des farouches guerriers de Malac, ils en conservent le langage, les mœurs, les habitudes, la religion et les lois: mais ils ont perdu, par I'habitude d'une longue soumission aux Portugais et aux Hollandais, une partie de la férocité de leurs ancêtres.

Cinquante-six individus de cette nouvelle race ont été le sujet de mes expériences : [...] je vais seulement discuter ici les principaux résultats de ces expériences. Par rapport à la différence des âges, on observe que la force devient presque double de 18 à 30 ans; qu'elle augmente très peu de 30 à 40 ; qu'elle diminue presque graduellement de 40 à 50 , de 50 à 60 : d'où l'on peut conclure que la période de 18 à 40 ans est effectivement celle qui, dans ces climats, présente la force de l'homme dans son plus grand développement. C'est dans cette classe que nous avons choisi les sujets de nos observations à la terre de Diémen, à la Nouvelle-Hollande, ainsi que parmi les Français et les Anglais : c'est aussi de cette classe que nous nous occupons exclusivement ici. Or, il résulte des expériences qui la concernent, 
$1^{\circ}$ Qu'aucun individu n'a fait preuve de cette vigueur remarquable qu'on observe assez souvent parmi les Européens :

$2^{\circ}$ Que presque tous se sont montrés très faibles:

$3^{\circ}$ Que le terme moyen de leur force rénale est de 16,2 myriagrammes:

$4^{\circ}$ Que celui de la force des mains est de 58,7 kilogrammes:

$5^{\circ}$ Que les Malais de Timor sont un peu plus forts, soit des reins, soit des mains, que les sauvages de la terre de Diémen et de la Nouvelle-Hollande:

$6^{\circ}$ Qu'ils sont beaucoup plus faibles, sous l'un et l'autre rapport que les Français et les Anglais, ainsi que nous allons l'observer.

\section{Section IV}

\section{Expériences sur la Force physique des Français}

CHEZ LES PEUPLES sauvages ou peu civilisés dont nous venons de parler, les observations dynamométriques offrent un caractère de rigueur et de généralité qu' on ne saurait leur donner jamais au milieu de nos sociétés européennes. Notre nombreuse population, la diversité de nos professions, de nos exercices, de nos aliments, de nos travaux habituels, etc., excluent toute comparaison exacte; et pour trouver le terme moyen de la force d'un peuple puissant et civilisé, comme le français, par exemple, il faudrait un nombre si considérable d'observations, il faudrait [190] les répéter sur tant de classes de la société, qu' on peut regarder un semblable résultat comme effectivement impossible à obtenir. Il n'en est pas ainsi des hordes sauvages, surtout de celles qui peuplent la Nouvelle-Hollande et la terre de Diémen: ici, la population est partout si faible, que les peuplades les plus nombreuses comptent à peine une centaine d'individus, et que la plupart en ont moins de cinquante: ici, toute différence de conditions, d'exercices, d'aliments, est inconnue : avec les mêmes besoins, avec les mêmes ressources, tous les individus ont les mêmes travaux à supporter, les mêmes privations à subir, les mêmes jouissances à partager. Cette uniformité, qui se reproduit dans tous les détails de leur existence, qui se 
soutient à toutes les époques de la vie, imprime aux individus un caractère de similitude physique et morale dont on aurait peine à se former une juste idée dans notre état social. Aussi n'hésité-je pas, malgré le petit nombre d'observations que je viens de présenter sur la force des habitants de la terre de Diémen, de la Nouvelle-Hollande et même de Timor, à en regarder les résultats comme beaucoup plus positifs que ceux qu' on pourrait tirer de séries d'expériences plus nombreuses faites sur des peuples européens. Je suis donc bien éloigné de vouloir ici, des observations dynamométriques que j'ai pu faire sur les Anglais et les Français, déduire aucune conséquence sur la force réelle des deux nations auxquelles appartiennent les individus [...] ; je les présente seulement comme un terme de comparaison d'autant plus propre à faire ressortir toute la faiblesse des peuples sauvages dont j'ai parlé, que ces expériences ont été faites dans les mêmes climats, sur des individus du même âge : elle reçoivent un nouvel intérêt de la différence de profession des hommes qu'elles comprennent; on y voit figurer, en effet, des négociants, des militaires, des juges, des naturalistes, des médecins, des matelots, etc. [...] La force manuelle de ces dix-sept Français est, terme moyen, de 69, 2 kilogrammes, et celle des reins, de 22, 1 myriagrammes; proportions bien supérieures l'une et l'autre à celle que nous avons successivement indiquées pour la terre de Diémen, la Nouvelle-Hollande et Timor. Nous allons revenir sur cette comparaison respective.

\section{Section $V$ \\ Expériences sur la Force physique des Anglais}

Sur quatorze Anglais du port Jackson [...], le résultat moyen est de 71, 4 kilogrammes pour les mains, et de 23, 8 myriagrammes pour les reins. Cette légère différence en plus pour les Anglais paraît dépendre en grande partie de celle qu'il y avait entre la santé des individus des deux nations, les Français revenant alors de courir les mers, et les Anglais se trouvant au contraire dans leurs foyers; elle peut d'ailleurs avoir été déterminée par tant d'autres petites circonstances locales 
ou individuelles, qu'il serait ridicule de vouloir en tirer quelque induction sur la force relative des uns et des autres.

[191]

\section{Section VI}

Résultats généraux des Expériences faites sur la Force physique des Sauvages de la terre de Diémen, de la Nouvelle-Hollande, des Habitants de Timor, des Français et des Anglais

EN RÉUNISSANT maintenant les résultats généraux des cinq séries d'expériences que je viens de rapporter, il s'ensuit, pour la force manuelle, les proportions suivantes, exprimées en kilogrammes:

Terre de Diémen

Nouvelle-Hollande

Timor

Français

Anglais
50,6

51,8

58,7

69,2

71,4

Pour la force des reins, les suivantes, exprimées en myriagrammes:

Terre de Diémen

Nouvelle-Hollande

Timor

Français

Anglais
14,8

16,2

22,1

23,8 
D'où il résulte,

$1^{\circ}$ Que les habitants de la terre de Diémen, les plus sauvages de tous, les enfants de la nature par excellence, sont les plus faibles:

$2^{\circ}$ Que ceux de la Nouvelle-Hollande, qui ne sont guère plus civilisés, sont plus faibles que les habitants de Timor:

$3^{\circ}$ Que ces derniers à leur tour sont beaucoup plus faibles, soit des reins, soit des mains, que les Anglais et les Français.

Nous pouvons donc déduire de l'ensemble de ces résultats la conséquence suivante:

Le développement de la force physique n'est pas toujours en raison directe du défaut de civilisation: il n'est pas un produit constant, il n'est pas un résultat nécessaire de l'état sauvage.

Telles sont les conséquences des observations que j'ai pu faire avec le dynamomètre pendant notre long et pénible voyage: je me proposais bien d'en faire de nouvelles au cap de Bonne-Espérance sur la race hottentote: mais l'intérêt de la science a dû me commander à l'Ile-de-France le sacrifice de l'instrument auquel je devais ce curieux travail. Je l'ai remis, avec l'autorisation du gouverneur de la colonie, à [192] M. Chapotin, médecin du gouvernement, et j'ai lieu d'espérer qu'entre ses mains il pourra fournir un jour les résultats les plus précieux. Nul pays, en effet, n'est aussi propre aux observations de ce genre que l'Ile-de-France: sur ce théâtre extrêmement resserré, l'activité de nos Européens réunit chaque jour les hommes des climats les plus divers: le Nègre des bords du Sénégal et du Niger, le farouche Yolof, le fier Malgache, le Caffre belliqueux et colossal, le robuste Mosambique, en un mot toutes les noires légions de l'A frique s'y trouvent confondues: on y rassemble et le Malais cruel sorti des rochers de Macassar, et l'habitant plus paisible des îles de la Sonde et des Moluques: les hommes indolents, faibles et doux qui puisent les eaux de l'Indus et du Gange, s'y trouvent en grand nombre, et quelques individus des îles du grand Océan commencent à s'y montrer : enfin, des 
milliers de navire y abordent annuellement de toutes les parties du monde... Quelle importance des observations dynamométriques dirigées avec intelligence, poursuivies avec opiniâtreté, ne recevraientelles pas, si on les étendait successivement à cette multitude de peuples si différents les uns des autres! Mais, sans rien préjuger sur un travail bien exécuté de ce genre, qu'il me soit permis de présenter maintenant quelques réflexions générales sur les causes présumées de la faiblesse des trois peuples dont j'ai parlé.

\section{Section VII}

Des Causes présumées de la Faiblesse des Habitants de la terre de Diémen, de la Nouvelle-Hollande et de Timor

JE VIENS de constater, par des expériences directes, un degré de faiblesse très remarquable dans les peuples de la terre de Diémen, de la Nouvelle-Hollande et de Timor: doit-on imputer exclusivement cette faiblesse à leur manière d'exister en société, ou même à l'absence de tout état social parmi eux ?... Ce problème, aussi difficile qu'intéressant, exigerait sans doute, pour qu'on pût en donner une solution rigoureuse, plus de faits que je n'en ai recueilli. Cependant ceux que j'expose ici me paraissant susceptibles de jeter un grand jour sur cette matière, j'ai cru devoir leur donner tout le développement que l'importance de la question exige.

Les physiologistes modernes se réunissent pour avouer que, toutes choses égales d'ailleurs, une nourriture abondante et salubre, un exercice habituel, continu, modéré surtout, sont les conditions les plus favorables au développement de la force physique, à son entretien. Une température un peu froide paraît être une troisième condition avantageuse, quoique moins générale et moins exclusive que les précédentes. Les dispositions contraires à celles que nous venons d'indiquer, ont été jugées devoir produire un effet opposé. Ce petit nombre de principes étant admis, les causes de la faiblesse des peuples dont nous parlons, doivent, ce me semble, paraître aussi simples qu'énergiques pour le prouver, il me suffira de retracer succinctement [193] l'état 
physique du sol sur lequel chacun de ces peuples se trouve placé par la nature.

\section{$1^{\circ}$ Habitants de Timor}

La grande île de Timor, trop peu connue des Européens et surtout des naturalistes, est, sous tous les rapports, I'un des pays les plus fertiles du monde. Il n'est pas de mon objet d'insister ici sur le riche tableau de ses productions végétales et animales; il me suffira de dire, en peu de mots, que toutes les espèces de fruits particulières au climat de l'Inde s'y trouvent réunies, sans qu'il en coûte à l'homme aucun soin de culture, aucun travail autre que celui de cueillir ces trésors: la nature fait tout le reste : le riz y est d'une qualité supérieu$r e$; les meilleures racines, la patate, l'igname, le manioc, et plusieurs autres qui me sont inconnues, y foisonnent de toute part : en un mot, le règne végétal paraît y avoir accumulé tous ses dons.

Dans le règne animal, même profusion de bienfaits. Autour de la case du Malais indolent, pullulent à l'envi des troupes de poules, de canards, de cochons, de chèvres, de moutons, de buffles, de chevaux, etc. Toutes les forêts de l'intérieur sont remplies de troupeaux considérables de la plupart de ces mêmes animaux redevenus sauvages. Ajoutez-y d'innombrables légions de singes et d'énormes chauvessouris dont les Malais aiment beaucoup la chair, qui est effectivement très tendre et très délicate; et l'on conviendra sans doute que, dans cette partie, l'habitant de ces régions n'a plus rien à désirer. Je ne parlerai pas des oiseaux, quelque abondants, quelque variés qu'ils puissent être, parce qu'au milieu de tant de richesses il les dédaigne absolument. Les rivières lui offrent en abondance des poissons délicats. Mais rien n'est comparable à la fécondité des rivages mêmes de Timor: tous les naturalistes de la capitale ont pu juger, par l'énorme quantité d'espèces d'animaux, poissons, crustacés, mollusques, testacés, zoophytes, etc., que nous avons rapportés M. Lesueur et moi, combien est prodigieuse la variété des êtres qui les peuplent. On peut à son gré se les procurer tous sans travail, sans instrument aucun : il suffit, à chaque marée basse, de descendre au rivage pour y prendre à 
la main une foule de ces animaux qui se trouvent délaissés dans de petits lacs trompeurs creusés au milieu des madrépores, et où ils se sont imprudemment oubliés à la retraite des flots. L'eau qui remplit ces trous, venant à filtrer à travers les lissures des madrépores, il s'y trouvent bientôt à sec, et présentent ainsi la proie la plus facile.

Du côté des aliments, nul peuple donc, il faut en convenir, n'a été plus favorisé que celui de Timor: excellence, abondance, diversité, tout se trouve réuni pour son usage. Sous ce rapport, il fut placé par la nature dans une des conditions les plus favorables au développement des forces physiques, à leur entretien.

Malheureusement cette facilité prodigieuse de satisfaire à tous les besoins de la vie, cette abondance de tous les biens sans mélange de peines et de labeurs, ont déterminé dans toute la nation un caractère d'apathie et d'indifférence si décidé, une [194] aversion si forte pour le travail et la fatigue, que l'idée seule de s'y livrer attristerait un Malais de ces régions. Demeurer accroupi une partie de la nuit et du jour, le derrière sur les talons, à l'ombre d'un tamarinier, d'un palmier, d'un bananier ou d'un manguier : mâcher sans cesse le bétel, boire du calou, faire trois ou quatre repas assez légers; toucher une sorte de guitare faite avec une feuille de latanier et un cylindre de bambou : dormir à diverses reprises du jour et de la nuit tresser quelques nattes, ou s'occuper d'autres ouvrages aussi légers, aussi faciles se baigner enfin, se peigner, se frictionner avec de l'huile de coco: tel est le cercle invariable des occupations d'un Malais libre à Timor. Pour les esclaves, ils sont en si grand nombre dans chaque maison, on exige d'eux si peu de travaux, ils les exécutent si lentement, que leur existence particulière n'est guère moins oisive que celle de leurs maîtres. On peut donc considérer ce premier peuple comme plongé dans un état permanent d'inaction ou de repos. Un tel genre de vie ne nous paraîtra-t-il pas, ainsi qu'à tous les physiologistes, une raison suffisante de cette faiblesse prouvée par le dynamomètre, et que l'inspection attentive de la conformation des individus ne démontre pas d'une manière moins positive? En effet, sans avoir les membres aussi faibles que les peuples de la terre de Diémen et de la Nouvelle-Hollande, les Malais de Timor les ont cependant peu développés et surtout peu musculeux: les formes en sont aussi beaucoup plus adoucies, les contours beaucoup 
plus gracieux que dans les individus européens: aussi leur nudité a-telle un caractère d'élégance que la nôtre ne saurait jamais offrir.

De ces observations, il résulte que l'inaction peut être considérée comme la cause essentielle du défaut de vigueur chez les habitants de Timor.

La température de l'île ne paraît pas non plus étrangère. Dans mon Mémoire sur la dysenterie des pays chauds et sur l'usage du bétel, j'ai particulièrement insisté sur l'action débilitante de l'atmosphère humide et chaude de l'île de Timor: j'ai prouvé, par notre trop funeste expérience, par l'expérience plus déplorable encore du navire américain The Hunter, combien sont rapides et meurtriers les effets produits par cette constitution atmosphérique : j'ai démontré que les habitants eux-mêmes n'étaient pas à l'abri de cette maligne influence, mais que, guidés par un instinct admirable, ils étaient parvenus de bonne heure à y opposer des moyens aussi simples qu'efficaces ; j'ai parlé de ces bains froids souvent réitérés, de ces frictions non moins fréquentes, par lesquels ils cherchent à redonner à la peau cette vigueur, cette énergie que la chaleur humide tend à détruire: j'ai fait connaître cette foule d'ingrédients aromatiques, amers, astringents, et surtout cette chaux vive, cet arreck, ce bétel, dont ils font usage pour ranimer intérieurement la tonicité de l'estomac et du canal intestinal. Toutes ces indications me semblent, à la vérité, parfaitement bien remplies: mais de telles pratiques n'en attestent pas moins une cause puissante d'affaiblissement général, dont l'action ne saurait être jamais parfaitement neutralisée, quelques remèdes qu'on pût d'ailleurs employer pour la combattre.

Ces derniers faits se trouvent d'accord avec les résultats obtenus par M. [195] Coulomb, dont le témoignage, si précieux par lui-même, reçoit un nouveau prix des nombreuses expériences qui les lui ont fournis.

«La quantité moyenne d'action, dit ce physicien célèbre, varie suivant le climat. J'ai fait exécuter de grands travaux à la Martinique par les troupes; le thermomètre y est rarement audessous de $20^{\circ}$ : j'ai fait exécuter en France les mêmes genres 
de travaux par ces mêmes troupes; et je puis assurer que le 14e degré de latitude, où les hommes sont presque toujours inondés de leur transpiration, ils ne sont pas capables de la moitié de la quantité d'action journalière qu'ils peuvent fournir dans nos climats. » (Mémoires de l'Institut, 1 ère Classe, Tome II, p. 429).

La haute température de Timor, son humidité habituelle, la vie indolente de ses habitants, me semblent donc fournir à elles seules une explication satisfaisante de la faiblesse particulière au premier de ces trois peuples dont nous avons parlé. Ici donc, il faut en convenir, le degré de civilisation ne paraît pas y influer d'une manière essentielle, immédiate. Il n'en est pas ainsi pour la terre de Diémen et la NouvelleHollande.

\section{$2^{\circ}$ Nouvelle-Hollande et Terre de Diémen}

La nature semble avoir traité les habitants de ces régions en marâtre. Le règne végétal n'y fournit presque rien: nous n'y avons jamais trouvé aucun fruit mangeable qui fût de la grosseur d'une cerise: on n'y connaît encore d'autres racines nutritives que celles de diverses fougères et quelques bulbes d'orchidées. Le règne animal, à son tour, n'offre d'espèces un peu considérables que le casoar et le kanguroo [sic], I'un et l'autre devenus très rares sur la grande terre, à cause de la chasse continuelle qu'on leur fait. La pêche pourrait, à la vérité, fournir aux habitants une ressource plus abondante, plus assurée: mais l'imperfection de leurs instruments et de leurs méthodes de pêche, I'hiver pour les habitants de la terre de Diémen, les orages fréquents pour la Nouvelle-Hollande, et surtout les émigrations des poissons, tout concourt à rendre cette dernière ressource trop souvent insuffisante et quelquefois même absolument nulle. C'est alors que se manifestent ces cruelles famines dont le gouverneur Philipp eut I'occasion lui-même d'observer les tristes effets peu de temps après son arrivée à la Nouvelle-Hollande. «Alors, dit M. Collins, on rencontrait les malheureux naturels réduits à un tel excès de maigreur, qu'on les 
eût pris pour autant de squelettes, et qu'ils paraissaient être sur le point de succomber d'inanition. " Les productions maritimes même ne sont d'aucun secours pour les peuplades repoussées dans l'intérieur des terres: ce sont celles-là surtout qui font une guerre active aux grenouilles, aux lézards, aux serpents, à diverses espèces de larves, et particulièrement à des grosses chenilles qui se réunissent autour des branches de l'eucalyptus résineux, et y forment des groupes de la grosseur de la tête. Les araignées elles-mêmes, comme à la NouvelleCalédonie, font partie de leurs repas dégoûtants: dans plusieurs circonstances, ces hordes misérables sont réduites à vivre de certaines herbes, à ronger l'écorce de différents arbres; enfin, il [196] n'est pas jusqu'aux fourmis nombreuses qui dévastent leur sol, qu'elles n'aient été contraintes de faire servir à leur nourriture. M. Collins a parlé de cette pâte horrible que les naturels préparent, en pétrissant ces insectes et leurs larves avec les mêmes racines de fougère dont je viens de parler: usage repoussant, dont la famine la plus hideuse a pu seule inspirer la première idée, et dont je ne crois pas qu'on ait trouvé la moindre trace dans tout le reste de l'univers.

Certes, de pareils aliments ne sont guère favorables au développement de la force physique; et sans doute il serait difficile de rencontrer ailleurs un peuple plus maltraité, sous ce rapport, que celui dont je parle.

Il en est de même de l'exercice. Au lieu de cette action modérée, continue, que l'expérience nous apprend être si propre à développer et à entretenir la vigueur, le sauvage dont il s'agit, entraîné par le besoin impérieux de se procurer des aliments pour apaiser la faim qui le presse, se livre pendant plusieurs jours à des courses longues et pénibles, ne prenant de repos que dans les instants où son corps tombe de fatigue et d'épuisement. Vient-il à trouver une pâture abondante; alors, étranger à tout mouvement autre que ceux qui sont indispensables pour qu'il puisse assouvir sa voracité, il n'abandonne plus sa proie; il reste auprès, jusqu'à ce que de nouveaux besoins le rappellent à de nouvelles courses, à de nouvelles fatigues non moins excessives que les précédentes. Or, quoi de plus nuisible au développement réel, à l'entretien harmonique des forces, que ces alternatives de fatigue outrée, de repos automatique, de privations accablantes, d'excès et d'orgies 
faméliques? Dans cette seconde partie du mode d'existence des peuples de la Nouvelle-Hollande et de la terre de Diémen, nous retrouvons donc encore une cause générale de la faiblesse extrêmement active, et qui se reproduit à toutes les époques de la vie de ces hommes malheureux.

Cette raison, jointe à la rareté des aliments, à leur disette même, et le plus souvent à la mauvaise qualité, ne pourrait-elle pas, sinon avoir primitivement déterminé, du moins avoir exagéré cette maigreur, cette émaciation excessive des extrémités de ces hommes, dont tous les voyageurs ont parlé avec étonnement, et sur laquelle j'aurai plus particulièrement l'occasion d'insister ailleurs? M. Labillardière, aux précieux travaux duquel il m'est bien doux d'avoir à payer un juste tribut d'éloges, avait observé déjà ce vice de conformation dans les habitants de la terre de Diémen: Cook avait fait la même remarque sur les peuples de la Nouvelle-Hollande, et, tout récemment, elle se reproduit dans l'ouvrage de M. Collins, sur les sauvages des environs de BotanyBay et du port Jackson. Ce même caractère s'observe d'une manière plus effrayante encore, à ce qu'il paraît, dans les sauvages de la terre de Feu, les misérables Pescherais, les seuls, pour le dire en passant, qu'on puisse avec raison comparer aux peuples de la terre de Diémen. "Leurs épaules, dit Forster, et leur estomac sont larges et osseux; le reste de leur corps est si mince et si grêle, qu'en en voyant séparément les diverses parties, nous ne pouvions nous persuader qu'elles appartinssent aux mêmes individus. » (Cook, 2e Voyage, T. VIII, p. 34).

Sans doute il ne serait pas invraisemblable de considérer un vice de conformation aussi général, aussi décidé, comme dépendant de l'organisation physique propre à des peuples qui, sous tant d'autres rapports, se distinguent de ceux déjà connus. Cependant, si l'on fait attention que, quelque communs qu'ils puissent être, on trouve néanmoins des individus doués de proportions beaucoup plus belles; si l'on se rappelle aussi que l'effet nécessaire de la disette, des fatigues excessives et des mauvais aliments, est de flétrir les formes, de dessécher I'habitude du corps, phénomène dont nous trouvons surtout un exemple remarquable dans ces Arabes du désert dont $M$. de Volney nous a fourni le premier la curieuse histoire, peut-être ne sera-t-on pas éloi- 
gné de penser que les vices de conformation propres aux peuples dont il s'agit, dépendent en grande partie de l'état de misère et de famine habituelle dans lequel ils vivent. C'est l'opinion de la plupart des Anglais instruits du port Jackson; elle est celle de M. Collins; et je dois l'avouer franchement, il me paraît difficile de se refuser à la partager.

De tout ce que je viens de dire, il semblerait donc résulter en dernière analyse, que le défaut d'aliments et leur mauvaise qualité, les fatigues indispensables pour les obtenir, peuvent être considérés comme les causes essentielles de la faiblesse des hommes de la Nouvelle-Hollande et de la terre de Diémen: mais cette disette d'aliments, cette nécessité de faire usage des substances les plus dégoûtantes, ces fatigues excessives pour se les procurer, ne peuvent-elles pas être considérées à leur tour comme un résultat immédiat et nécessaire de l'état sauvage dans lequel ces peuplades malheureuses végètent encore ?... C'est ce que le raisonnement le plus rigoureux et l'analogie la plus irrécusable semblent devoir consacrer.

En effet, supposons pour un instant que ces enfants déshérités de la nature viennent à déposer leurs mœurs féroces et vagabondes: supposons que, réunis en tribus plus nombreuses, ils se rassemblent dans des villages: que tous ensemble conviennent de mettre un terme à ces guerres éternelles et sanguinaires qui dévorent la population: supposons que le droit de propriété vienne exciter au milieu d'eux une heureuse émulation: que la violence et la force de l'individu, réprimées par la force de tous, aient cessé d'être l'unique règle de la conduite de chacun; en un mot, supposons-les pour un instant au même degré de civilisation que les Kamtschadales ou les Samoïèdes : ce n'est pas beaucoup exiger, sans doute; et cependant de quels changements heureux cette seule différence d'organisation sociale ne va-t-elle pas devenir le principe? Combien les ressources de l'homme ne vont-elles pas se multiplier! Combien ne va-t-il pas se trouver loin de ce dénuement déplorable dans lequel il traîne maintenant sa précaire existence!

Déjà ne croit-on pas voir les diverses espèces de kanguroos, devenues domestiques, pulluler autour de sa cabane? Le casoar, qui, plus facilement encore, se prête aux soins de l'homme, va lui présenter 
journellement sa chair abondante et délicate, ses œufs volumineux et de très bon goût. Le cygne noir multipliera dans toutes les pièces d'eau voisines. Le beau faisan à queue de lyre, le superbe menura [198] des Montagnes Bleues, annonce assez par la famille à laquelle il appartient, de quels avantages il pourrait payer les soins de l'homme.

Bientôt ses arts perfectionnés par le loisir, et surtout par cette heureuse communauté d'idées et d'efforts que la société seule rend possible, vont lui fournir des instruments de pêche plus variés, plus parfaits. Avec beaucoup moins de temps, avec beaucoup moins de peine, il obtiendra de ses rivages des produits plus abondants. Il ne tardera pas à découvrir le moyen de se ménager, par la dessiccation, par la salaison, des ressources certaines pour les temps d'hiver ou d'orages, pour ceux encore où les poissons doivent se retirer vers d'autres plages. Alors aussi, son canot, mieux travaillé, lui permettra d'étendre sa navigation: les îles voisines ne lui seront plus étrangères; les innombrables légions de manchots et de phoques qui les peuplent, seront pour lui une proie aussi facile qu'inépuisable; leur huile va donner une nouvelle saveur à ses aliments; leurs fourrures lui procureront un abri plus puissant contre les vicissitudes de l'atmosphère; la belle oie du détroit de Bass sera bientôt réunie dans sa basse-cour. De ces mêmes îles, sans doute, il rapportera le wombat ou phascolome, cet animal singulier, désagréable à la vue, mais dont la chair est si tendre, si délicate, et que nous avons trouvé nous-mêmes aussi familier qu'un chien dans les cabanes des pêcheurs anglais. Le kanguroo gris, dont la chair est bien meilleure que celle du géant, sera l'une des précieuses acquisitions que l'homme pourra faire encore dans ces îles, et cette dernière ne sera pas une des moins importantes.

Certes, il n'y a rien de forcé dans le tableau que je viens de tracer ici des biens que l'homme peut rassembler en très peu de temps; et cependant combien déjà tout est changé pour lui! Sa subsistance est assurée pour toujours; elle est indépendante des saisons, de l'approche ou de la retraite du poisson; elle est plus abondante, elle est surtout de meilleure qualité. Dès ce moment, étranger à ces privations cruelles, à ces fatigues excessives, à ces courses accablantes et réitérées qui consument sa vigueur, qui flétrissent son existence, l'homme, sans doute, verra bientôt son tempérament devenir plus robuste: sa 
force croîtra dans le même rapport; et s'il est vrai, comme tout semble l'indiquer, que les vices de sa conformation soient un des funestes effets de l'état habituel de famine et de misère dans lequel il vit maintenant, ne peut-on pas pressentir que cette émaciation hideuse doit être insensiblement remplacée par des formes moins arides, moins flétries?

Ces modifications heureuses ne seront pas bornées à l'individu luimême: la société ne tardera pas à en partager les effets salutaires. La population, si faible aujourd'hui, fera des progrès rapides: on ne verra plus, comme on le voit maintenant sur ces tristes bords, des femmes réduites à se faire avorter par les moyens les plus cruels, pour ne pas donner l'existence à des enfants qu'elles craignent, avec raison, de ne pouvoir pas nourrir ; on ne verra plus des pères, forcément dénaturés, écraser avec de grosses pierres, sur le corps de leur mère qui vient de mourir, ces pauvres orphelins qu'elle délaissa trop jeunes, parce qu'ils ne sauraient ni les nourrir, [199] ni les traîner dans leurs courses lointaines. M. Collins, dont j'ai déjà cité l'ouvrage, rapporte cette dernière coutume avec les mêmes détails que je viens d'indiquer. Nous en avons nous-mêmes été instruits, sur les lieux, par les personnes les plus recommandables; et si l'on veut réfléchir sur les particularités de l'existence de ces peuples, on ne tardera pas à reconnaître que ces actions barbares en sont une conséquence effroyable sans doute, mais nécessaire... Triste prérogative de cet état de nature, tant préconisé naguère, que de justifier, ou même de légitimer ces horribles forfaits, que les avantages de la civilisation rendent à peine vraisemblables pour nous... 


\section{Section VIII}

Résultats généraux

DE L'ENSEMBLE des faits que j'ai successivement exposés dans ce mémoire, on peut déduire les résultats généraux suivants :

$1^{\circ}$ Les habitants de Timor, de la Nouvelle-Hollande et de la terre de Diémen, incomparablement moins civilisés que les Français et les Anglais, sont néanmoins beaucoup plus faibles qu'eux.

$2^{\circ}$ La température humide et chaude de l'île de Timor, et surtout la vie trop indolente, trop inactive de ses habitants, paraissent devoir être considérées comme les causes essentielles de la faiblesse de ces peuples.

$3^{\circ}$ À la terre de Diémen, à la Nouvelle-Hollande, le défaut d'aliments, leur mauvaise qualité, les fatigues excessives auxquelles I'homme est contraint de se livrer pour les obtenir, semblent être en même temps les causes primitives de sa faiblesse et des vices de sa conformation.

$4^{\circ}$ Mais j'ai prouvé que cette disette habituelle dépend exclusivement de l'état sauvage dans lequel ces peuples végètent encore: donc cet état-là même, proclamé si longtemps comme la source de la puissance physique et de la vigueur, devient ici le principe d'une faiblesse excessive.

$5^{\circ} \mathrm{J}$ 'ai pareillement prouvé qu'il était probable que le perfectionnement de l'état social, en rendant l'homme de ces contrées à l'abondance, pourrait déterminer un développement plus considérable des forces physiques, et faire disparaître les vices de sa conformation actuelle: donc les progrès de la civilisation deviendraient ici la double source de la vigueur et de la perfection physique.

$6^{\circ}$ Que penser désormais de ces éloquentes déclamations contre le perfectionnement de l'ordre social, déduites de la force 
extraordinaire de l'homme sauvage, ou plutôt, pour parler le langage des sophistes, de I'homme de la nature?...

Je me garderai cependant bien de vouloir donner à ces résultats de mon travail sur ce sujet plus d'extension qu'ils n'en doivent naturellement avoir. J'ai prouvé que le problème de la force physique des peuples, même les plus grossiers, n'est pas [200] aussi simple qu' on aurait plu le croire d'abord; que, d'une part, il se rattache essentiellement aux détails de leur constitution physique, de leur organisation sociale, et que, de l'autre, il se trouve lié par les rapports les plus puissants avec I'histoire du climat, de sa température, de ses productions diverses, etc. Envisagée sous ce rapport, la question que je viens de traiter est bien loin de pouvoir être résolue d'une manière générale... Il me suffit d'avoir le premier en ce genre ouvert la carrière de l'observation, et d'avoir opposé des expériences directes, des faits nombreux à cette opinion trop communément admise, trop dangereuse peut-être, et bien certainement trop exclusive, de la dégénération physique de I'homme par le perfectionnement de la civilisation... 
[201]

Aux origines de l'anthropologie française. (1994)

Édition revue et corrigée par les auteurs, 1993.

Indications biographiques sommaires

Nicolas Baudin (1750-1803)

Retour à la table des matières

APRÈS DES DÉBUTS DIFFICILES dans la marine marchande, Baudin fut nommé lieutenant de vaisseau dans la marine royale en 1786 , et participa à plusieurs expéditions scientifiques (Indes, Antilles). Grâce aux collections botaniques et zoologiques qu'il avait constituées lors de ses voyages, et qui lui valurent l'estime et la reconnaissance des professeurs du Muséum d'Histoire naturelle, le Directoire le promut capitaine de vaisseau. Il lui confia le commandement des deux corvettes Le Naturaliste et Le Géographe, qui devaient partir en exploration " aux terres australes» suivant un programme que Baudin avait luimême soumis à l'Institut de France et qui fut agréé par une commission à laquelle participèrent notamment Bougainville, Lacepède et Jussieu. Baudin est mort le 16 septembre 1803 à l'île de France (actuellement île Maurice) des suites d'une maladie contractée au cours du voyage. Il ne laissa que des documents manuscrits: le journal de bord de l'expédition (conservé aux Archives nationales) patiemment mis au point, traduit en anglais et publié par l'université d'Adélaïde en Australie (cf. bibliographie), et quelques lettres adressées à A.-L. Jussieu en 1802 et 1803, toutes inédites, actuellement conservées au Muséum 
national d'Histoire naturelle de Paris. Longtemps présenté comme un homme autoritaire et incapable (par François Péron notamment), Baudin n'a été reconnu, sa personnalité et son œuvre réévaluées, qu'au milieu du XXe siècle, grâce aux travaux de J.-P. Faivre; un colloque récent lui $a$, de ce point de vue, rendu justice : cf. A. Dommergues et M. Nedeljkovic (éds.), Les Français et l'Australie. Voyages de découvertes et missions scientifiques de 1796 à nos jours, Paris, Le Havre, Université Paris X-Nanterre, 1989. Dans le numéro spécial que la revue L'Ire des vents $\left(n^{\circ} 3-4,1981\right)$ a publié en hommage à Michel Leiris en 1981, celui-ci a tenu à voir figurer les extraits de la correspondance de Baudin à Jussieu que nous avons reproduits sans ce livre.

\section{Georges Cuvier (1769-1832)}

ISSU D'UNE FAMILLE PROTESTANTE de Montbéliard, Georges Cuvier fit de brillantes études à l'Académie de Stuttgart, devint ensuite précepteur dans une famille de Normandie, puis passa les années de la Révolution dans la campagne du pays de Caux où, rapporte-t-on, le contact avec la nature fut à l'origine de sa vocation. En 1799, il succéda à Daubenton au Collège de France; en 1802, il devint professeur au Muséum d'histoire naturelle; en 1803, il fut élu secrétaire perpétuel [202] de l'Académie des sciences pour les sciences physiques et naturelles; et en 1813, il devint maître des requêtes au Conseil d'État, peu de temps après Gérando qu'il connut en tant que membre de la Société des observateurs de l'homme. La Restauration le vit successivement conseiller d'État, chancelier de l'Instruction publique, et en 1831, pair de France. Il mourut en 1832 en laissant une œuvre dont l'importance et l'influence ne peuvent être ici envisagée 200.

200 Sur celles-ci, on pourra consulter entre autres, l'ouvrage de F. JACOB, la Logique du vivant, une histoire de I'hérédité, Paris, Gallimard, 1970, 


\section{Joseph-Marie de Gérando (1772-1842)}

APRÈS UN BREF PASSAGE dans les ordres et dans les armes, Gérando fut lauréat de l'Institut en 1799 grâce à un mémoire qu'il présenta sur «les signes». Venu à Paris, il entreprit une brillante carrière administrative et politique et, en 1804, fut nommé secrétaire général du ministère de l'Intérieur. En 1808, il devient maître des requêtes au Conseil d'État, en 1810, conseiller d'État, en 1812, intendant de la Catalogne. Élu à l'Académie des sciences morales et politiques en 1832, il accède à la pairie en 1837. Très lié avec les philosophes et les Idéologues de son époque. Gérando - dont l'orthographe de son non n'a jamais été définitivement établie, se voyant tantôt nommé Degérando, tantôt de Gérando, tantôt Gérando - a été membre de plusieurs sociétés savantes, et, simultanément, administrateur de l'hospice des Quinze-Vingt, de l'Institution des sourds-muets, vice-président du Conseil de santé, fondateur et président de la Société d'encouragement pour l'industrie nationale. En plus des Considérations...., Gérando a laissé des ouvrages de philosophie largement inspirés de Locke et Condillac: Des signes et de l'art de penser considérés dans leurs rapports mutuels, Paris, Goujon et fils, 1880, 4 vol. (ouvrage qui reprend et développe son mémoire sur les signes primé par l'Institut en 1799); ainsi que De la génération des connaissances humaines, Berlin, Decker, 1802, qui, à l'origine, fut un mémoire qui obtint le prix de l'Académie de Berlin, ainsi que des textes «philanthropique » tel que le célèbre Visiteur du pauvre, récemment réimprimé aux éditions Jean-Michel Place dans la collection « Les cahiers de Gradhiva ».

\section{Louis-François Jauffret (1770-1850)}

MALGRÉ L'ÉTUDE DE REBOUL (cf. bibliographie), la vie et l'œuvre de Louis-François Jauffret restent largement méconnus. La « provincialité » de la deuxième moitié de sa vie (proviseur au lycée de Montbrison, puis en 1823, secrétaire de la faculté de droit d'Aix, enfin bi- 
bliothécaire et secrétaire de l'Académie de Marseille), la notoriété de son frère ainé, le prélat Gaspard-Jean [203] Jauffret (1759-1829), éclipsèrent en partie son œuvre et son rôle. Il manifesta cependant, tant par les cours d'histoire naturelle de l'homme qu'il professa au Louvre puis au Muséum dans les années 1800 que par ses mémoires ou que par l'impulsion qu'il sut donner à Société des observateurs de I'homme dont il devint le secrétaire perpétuel, un esprit de synthèse, une curiosité et une rigueur scientifiques qui en font un précurseur méconnu des sciences de l'homme, et qui le placent parmi les premiers à s'être interrogés sur les questions de méthodes posées par l'observation des sociétés humaines. On lui doit un ouvrage savoureux sur Les Charmes de l'enfance et les plaisirs de l'amour maternel, Paris, Moutard, 1791, qui eut un certain succès. Ce livre fut d'ailleurs offert par Jauffret au Chinois Tchong-A-Sam qu'il connut et «observa» avec Leblond en 1800. Dans son cours édité par Georges Hervé (cf. bibliographie), il raconte cet épisode: «Je lui fis présent à son départ de mon ouvrage sur Les Charmes de l'enfance et les plaisirs de l'amour maternel. Ce livre, si analogue aux mours de la Chine, lui fut une preuve de l'intérêt qu'il m'avait inspiré. Il fut sensible à cette attention, quoiqu'il ne pût rien entendre à l'ouvrage; et il m'exprima très affectueusement qu'il le conserverait toujours avec soin » (p. 276). La majeure partie des cours et des mémoires de Jauffret $n$ 'ont jamais été publiés.

\section{François Péron (1775-1810)}

APRÈS TROIS ANS D'ÉTUDES à l'École de médecine de Paris et une formation de naturaliste acquise auprès de Cuvier, François Péron fut recruté, grâce à la lecture de son mémoire sur l'anthropologie devant les membres de ]'Institut comme zoologiste et anthropologiste dans l'équipe scientifique de l'expédition du capitaine Baudin. D'un caractère entier, mais doué d'une imagination et d'une facilité d'écriture peu communes, Péron se heurta très tôt au réalisme et à l'empirisme de Baudin, et contribua grandement à envenimer les rapports entre l'équipage et le personnel scientifique de l'expédition, dont il devint «l'enfant terrible». Promu correspondant de l'Institut en 
1805, Péron rédigea la première partie du rapport de l'expédition, partie où il se laissa gagner par son animosité à l'égard de Baudin, faisant de ce dernier le «rustre » que la littérature des voyages a souvent dépeint. Mort en 1810, à trente-cinq ans, des suites d'une maladie contractée au cours du voyage, Péron ne put mettre un terme au rapport (continué et achevé par L.-C. Freycinet), ni à son projet de « tableau général des races humaines ». Son amitié avec le dessinateur Lesueur permit de retrouver, à sa mort, dans les papiers de celui-ci, la note manuscrite de Cuvier, spécialement rédigée à son intention.

[204]

\section{Philippe Pinel (1745-1826)}

SUCCESSIVEMENT MÉDECIN-CHEF de Bicêtre (1793), médecinchef de la Salpêtrière (1795), membre de l'Institut (1803), puis médecin consultant de Napoléon (1805), Philippe Pinel est surtout connu pour avoir prétendument « adouci » la relation thérapeutique dans les hôpitaux psychiatriques et conçu des principes d'organisation de l'institution (la «police »psychiatrique), ainsi que pour avoir défini une classification des maladies fondée non plus sur les symptômes mais sur les organes lésés, suivant en cela une méthode apportée par des naturalistes tels Linné et Jussieu et par des anatomistes comparatistes tel Cuvier. Considéré comme le pionnier de la psychiatrie européenne, Pinel resta étroitement lié aux Idéologues de l'époque dont il s'inspira en partie, ne s'embarrassant guère des contradictions philosophiques qui le virent osciller entre l'empirisme sensualiste de Condillac et le spiritualisme, en passant par le matérialisme de Cabanis. Ses principaux travaux sont: Traité médico-philosophique sur l'aliénation mentale, Paris, 1801 ; et Nosographie philosophique, Paris, 1813. 


\section{Société des observateurs de l'homme (1799-1805)}

FONDÉE EN 1799, cette Société était composée d'une soixantaine de membres qui se répartissaient, par formation et par discipline, comme suit: Naturalistes: Cuvier, Jauffret, Jussieu, Virey : Linguistes: Destutt de Tracy, Gérando, Leblond, Massieu, Sylvestre de Sacy, Sicard: Philosophes : Laromiguière, Lemoreau, Moreau, Garat : Ecclésiastiques : (outre Sicard), Jauffret (le frère de L.-F. Jauffret) ; Médecins: Bouvoyer-Desmortiers, Bojanus, Cabanis, Duméril, GuillonPastel-Hallé, Itard, Lassus, Moreau de la Sarthe, Nysten, Pinel, Thouret. Sue: Explorateurs: Andrew, Baudin, Bernier, Bougainville, Bissy, Hamelin (qui fut le second de l'expédition Baudin), Legout, Levaillant, Maugé, Michaux, Riedlé, Sonnini : Archéologues, historiens économistes : d Ansse de Villoison, Bouchaud, Clermont-Lodève, Larcher, Marcel, Millin, Papou, Pastoret, Pfeffel, Portalis, Sainte-Croix, Volney, Walckenaer: Hellénistes: Coray, Ricard, d'Ansse de Villoison. ; Publicistes et divers: Bonnefoux, La Chaussée, Lerminier, MathieuMontmorency, Haller. Sous la présidence de Jussieu - avec Jauffret comme secrétaire perpétuel - la Société fut chargée par l'Institut - à l'instigation de Lacépède - de préparer l'expédition de Baudin et d'en rédiger les instructions scientifiques. La Société devait disparaître en 1805, privée, par les guerres et le Blocus Continental, de documents anthropologiques, mais aussi politiquement divisée en son sein depuis la proclamation de l'Empire. Dans un des derniers procès-verbaux, celui du 7 juin 1804, Jauffret avait suggéré que la Société prît le nom de "Société impériale des observateurs de l'homme ». Cette proposition $n$ 'obtint pas le consentement de ses membres : elle fut finalement dissoute en 1805, les membres restants se fondant avec la Société philanthropique. 
[205]

\author{
Aux origines de l'anthropologie française. (1994) \\ Édition revue et corrigée par les auteurs, 1993. \\ Bibliographie des ouvrages cités
}

Retour à la table des matières

ALTHABE, G., D. FABRE, G. LENCLUD, (éds.), Vers une ethnologie du présent, Paris, éditions de la Maison des Sciences de l'Homme, 1992.

AMSELLE, J.-L., (éd.), Le Sauvage à la mode, Paris, Le Sycomore, 1979.

BAUDIN, N., The Journal of Post Captain Nicolas Baudin, Adélaïde, Libraries Board of South Australia, 1974 (Préface de J.-P. Faivre).

BONWICK, J., Daily Life and Origin of the Tasmanians, Londres, Sampson Low, 1870.

BONWICK, J., The Last of the Tasmanians, or the Black-War of Van Diemen's Land, Londres, Sampson Low, 1870.

BOUDIN, « Séance du 2 janvier 1862 de la Société d'anthropologie de Paris », Bulletins de la Société d'anthropologie de Paris, T. III, 1862, pp. 1-14.

BOUDON, R., L'Idéologie ou l'origine des idées reçues, Paris, Fayard, 1986. 
BOUTEILLER, M., «La Société des observateurs de I'homme, ancêtre de la Société d'anthropologie de Paris», Bulletins et Mémoires de la Société d'anthropologie de Paris, 10ème série, 1956, pp. 448465.

BRAUNSTEIN, J.-F., « Le sourire du pâle Vasco ou le voyage des Idéologues », Oui, la philosophie, $n^{\circ} 3,1984$, pp. 46-55.

BRAUNSTEIN, J.-F., « De Gérando, le "social" et la fin de l'Idéologie », Corpus, n 14-15, 1988, pp. 197-215.

BROCA, P., «Histoire des travaux de la Société (1859-1863)», Mémoires de la Société d'anthropologie de Paris, T. Il, 1865, pp. VIILI.

BROCA, P., Histoire du progrès des études anthropologiques depuis la fondation de la Société, séance solennelle du 8 juillet 1869, Paris, Hennuyer, 1870.

BROCA, P., "Leçon d'ouverture des cours publics d'anthropologie du 15 novembre $1876 »$, Revue d'anthropologie, 1877, pp. 172-182.

BROCA, P., Mémoires d'anthropologie, Paris, Jean-Michel Place, 1989 (réimpression de l'édition de 1871; préface de Claude Blanckaert).

BROSSES, C., (de), Traité de la formation mécanique des langues et des principes physiques de l'étymologie, Paris, Saillant, 1765, 2 vol.

BROSSES, C., (de), Du Culte des dieux fétiches, Paris, 1760 (réimpression, Paris, Fayard, 1988).

BUCHER, B., La Sauvage aux seins pendants, Paris, Hermann, 1977.

CASS, L., Inquiries, respecting the History, Traditions, Languages, Manners, [206] Customs, Religion, etc., of the Indian living within the United States, Detroit, Sheldon and Reed, 1823.

CASTEL, R., L'Ordre psychiatrique. L'âge d'or de l'aliénisme, Paris, Minuit, 1976.

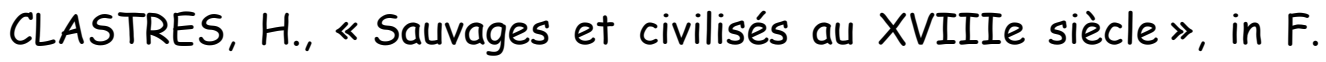
CHATELET, Histoire des idéologies, T. III, Paris, Hachette 1978, pp. 209-228. 
CLINE, H. \& AL., « The Relaciones geograficas of the Spanish Indies and New Spain, 1577-1792 », in Guide to Ethnohistorical Sources, part 1, Handbook of Middle American Indians, $n^{\circ} 12$, pp. 183-449.

CONDORCET, M.-J., Esquisse d'un tableau historique des progrès de l'esprit humain, Paris, Agasse, 1794. [Livre disponible dans Les Classiques des sciences sociales. JMT.]

COPANS, J., «Les Tendances de I'anthropologie française», in Questions à la sociologie, Paris, P.U.F., 1976, pp. 45-60.

COPANS, J., «A la recherche de la théorie perdue : marxisme et structuralisme dans I'anthropologie française », Anthropologie et sociétés, Vol. Ier, n 3, 1977, pp. 137-158.

COPANS, J., The French Revolution and the Black People of and from Africa, Travaux et documents, $n^{\circ}$ 4, Nairobi, Credu, janvier 1990.

COURT DE GÉBELIN, Histoire naturelle de la parole, Paris, 1776.

COTTEN, J.-P., « La politique de la philosophie. Note sur la France du début du XIXe siècle », La Pensée, n² 274, 1990, pp. 77-86.

DESCOLA, PH., G. LENCLUD, C. SEVERI \& A. C.TAYLOR, Les Idées de l'anthropologie, Paris, Armand Colin, 1988.

DÉSIRAT, C. \& T. HORDÉ, Du bon usage du sens. Textes linguistiques et ethnographiques de Volney, s. 1. n. d. [1980].

DÉSIRAT, C. \& T. HORDÉ, (éds.), « Les Idéologues et les Sciences du langage $», n^{\circ}$ spécial de: Histoire, épistémologie et langage, T. 4, fasc. 1, 1982.

DESTUTT DE TRACY, Eléments d'idéologie, Paris, Vrin, 1970 (réimpression de l'édition de 1801).

DIAS, N., Le Musée d'ethnographie du Trocadéro (1878-1908). Anthropologie et muséologie en France, Paris, éditions du CNRS, 1991.

DONZELOT, J., La Police des familles, Paris, Minuit, 1977.

DUCHET, M., Anthropologie et histoire au Siècle des Lumières Paris, Maspéro, 1971; réédité sous le même titre chez Flammarion en 1977. 
DUNMORE, J., French Explorers in the Pacific, Londres, Oxford University Press, 1969, vol. II, (cf. Baudin : pp. 9-40).

EDWARD, W. F., Esquisse de l'état actuel de l'anthropologie ou de I'histoire naturelle de I'homme, Paris, Imprimerie Dondey-Dupré, 1841.

EDWARD, W. F., De l'influence des races sur le caractère national, Paris, [207] Imprimerie Dondey-Dupré, 1945.

FAIVRE, J.-P., L'Expansion française dans le Pacifique. 1800-1842, Paris, Nouvelles éditions latines, 1953.

FAIVRE, J.-P., « Les Idéologues de l'an VIII et le voyage de Nicolas Baudin en Australie (1800-1804)», Australian Journal of French Studies, vol. III, $n^{\circ} 1,1966$, pp. 3-15.

FAIVRE, J.-P., J. POIRIER, P. ROUTIER, La Nouvelle-Calédonie.... Paris, Nouvelles éditions latines, 1955.

FOWLER, D., « Notes on Inquiries in Anthropology: a bibliographical Essay », in T. H. H. THORESEN (éd.), Towards a Science of Man. Essays in the History of Anthropology, Mouton, La Haye, 1975, pp. 1532.

FOUCAULT, M., Histoire de la folie à l'âge classique, Paris, Plon, 1961.

FOUCAULT, M., Les Mots et les choses, Paris, Gallimard, 1966.

GAULMIER, J., L'Idéologue Volney, Beyrouth, 1951.

GAULMIER, J., (éd.), [VOLNEY], Voyage en Égypte et en Syrie, Paris, Mouton, 1959.

GAULMIER, J., (éd.), [VOLNEY], La Loi naturelle. Leçon d'histoire, Paris, Garnier, 1980.

GÉRANDO, J.-M. (de), Considérations sur les diverses méthodes à suivre dans l'observation des peuples sauvages, Paris, Société des observateurs de l'homme, an VIII (1799). 
GÉRANDO, J.-M. (de), Des signes et de l'art de penser considérés dans leurs rapports mutuels, Paris, Goujon fils, 1799, 4 vol.

GÉRANDO, J.-M. (de), De la génération des connaissances humaines, Berlin, Decker, 1802.

GÉRANDO, J.-M. (de), Le Visiteur du pauvre, Paris, L. Colas, 1824 (réimpression, Paris, Jean-Michel Place, 1990, avant-propos de J. Ja$\min )$.

GÉRANDO, J. -M. (de), De la bienfaisance publique, Paris, Renouard, 1839, 2 vol.

GINESTE, T., Victor de l'Aveyron, dernier enfant sauvage, premier enfant fou, Paris, Le Sycomore, 1981, (réédition revue et augmentée, Paris, Hachette, 1993).

GIRARD, M., François Péron, naturaliste, voyageur aux terres australes..., Paris, Baillères, 1857.

GRIAULE, M., Méthode de l'ethnographie, Paris, P.U.F., 1957.

GRUBER, J., «Ethnographic Salvage and the Shaping of Anthropology », American Anthropologist, vol. LXXII, 1970, pp. 1289-1299.

GUICHETEAU, T. \& J.-P. KERNEIS, «Étude à travers les chrononavigrammes des responsabilités médicales de Baudin en Australie en 1801-1803 », in Les Français et I'Australie, Paris, Université Paris XNanterre, 1989, pp. 141-156.

GUSDORF, G., «Ethnologie et métaphysique: I'unité des sciences humaines, in J. Poirier (éd.), Ethnologie générale, Paris, Gallimard, 1968, pp. 1172-1815.

[208]

GUSDORF, G., La Conscience révolutionnaire. Les Idéologues, Paris, Payot, 1978.

HADDON, A. C., History of Anthropology, Londres, Watts, 1949.

HALE, K., « Some Questions about Anthropological Linguistics: the Role of Native Knowledge », in D. HYMES (éd.), Reinventing Anthropology, New York, Pantheon Book, 1973. 
HAMY, E.-T., Les Origines du musée d'ethnographie, histoire et documents, Paris, Ernest Leroux, 1890 (réimpression Paris, JeanMichel Place, 1989 : préface de N. Dias).

HAMY, E.-T., «L'œuvre ethnographique de Nicolas-Martin Petit, dessinateur à bord du Géographe, 1801-1804 », L'Anthropologie, T. II, 1891, pp. 601-622.

HAMY, E.-T., Les Humboldt et les Gérando; à propos de quelques autographes de W. et Al. de Humboldt, Lyon, Imprimerie Rey, 1906.

HAMY, E.-T., «Les Collections anthropologiques et ethnographiques du voyage de découvertes aux terres australes (1801-1804)», Bulletin de géographie historique et descriptive, $n^{\circ} 1,1906$, pp. 24-34.

HENSON, H., British Social Anthropologists and Language, Oxford, Clarendon Press, 1974.

HERVÉ, G., « Les débuts de l'ethnographie au XVIIIe siècle (17011765) », Revue de l'École d'anthropologie de Paris, 1909, pp. 345-366, et pp. 381-401.

HERVÉ, G., «Le Chinois Tchong-A-Sam à Paris», Revue de l'École d'anthropologie de Paris, 1909, pp. 171-179. [Présentation et publication du mémoire de Jauffret et Leblond : Le Chinois Tchong-A -Sam].

HERVÉ, G., «Le premier programme de l'ethnologie», Bulletins et Mémoires de la Société d'anthropologie de Paris, T. X, 5e série, 1909, pp. 473-487. [Présentation et publication du mémoire de L.-F. Jauffret: Introduction aux mémoires de la Société des observateurs de I'homme].

HERVÉ, G., «À la recherche d'un manuscrit. Les instructions anthropologiques de G. Cuvier pour le voyage du Géographe et du Naturaliste aux terres australes ", Revue de l'École d'anthropologie de Paris, 1910, pp. 289-306. [Présentation et publication du mémoire de $G$. Cuvier: Note instructive sur les recherches à faire relativement aux différences anatomiques des diverses races d'hommes].

HERVÉ, G., «Le Sauvage de l'Aveyron devant les observateurs de I'homme», Revue anthropologique, vol. XXI, 1911, pp. 383-398, et pp. 411-454. [Présentation et publication du mémoire de Ph. Pinel : Rapport 
fait à la Société des observateurs de l'homme sur l'enfant connu sous le nom de sauvage de l'Aveyron].

HERVÉ, G., «Les premières armes de Péron », Revue anthropologique, vol. XXIII, 1913, pp. 1-16. [Présentation et publication du mémoire de F. Péron: Observations sur I'anthropologie...],

[209]

HERVÉ, G., « Aoutourou ou le Tahitien à Paris», Revue anthropologique, vol. XXIV, 1914, pp. 207-219.

HERVÉ, G., «Les premiers cours d'anthropologie», Revue anthropologique, vol. XXIV, 1914, pp. 255-276. [Présentation et publication de la onzième leçon de L.-F. Jauffret].

HONIGMAN, J. (éd.), Handbook of Social and Cultural Anthropology, Chicago, Rand Mc Nally, 1973.

HONIGMAN, J. (éd.), The Development of Anthropological Ideas, Homewood, The Dorsey Press, 1976.

HORNER, F., « The Baudin Expedition to Australia (1800-1804) » in Les Français et l'Australie, Paris, Université Paris X-Nanterre,1989, pp. 107-114.

JACOB, F., La Logique du vivant. Une histoire de I'hérédité, Paris, Gallimard, 1970.

JAMIN, J., «L'indigent, l'indigène et les Idéologues», Anthropologie et sociétés, vol. 3, n², 1979, pp. 55-80.

JAMIN, J., « Le syndrome chinois des Idéologues ou les débuts de la sociolinguistique », Histoire, épistémologie et langage, IV, $n^{\circ} 1$, 1982, pp. 83-92.

JAMIN, J., «Faibles sauvages... Corps indigènes, corps indigents. Le désenchantement de François Péron», in J. HAINARD et $R$. KAEHR, Le Corps enjeu, Neuchâtel, Musée d'ethnographie, 1983, pp. 45-76.

JAMIN, J., « Note sur le dynamomètre de Régnier », Gradhiva, $n^{\circ}$ 1,1986, pp. 17-21. 
JAMIN, J., "L'histoire de l'ethnologie est-elle une histoire comme les autres? », Revue de synthèse, $n^{\circ} 3-4,1988$, pp. 469-483.

JAMIN, J., « Anthropologie française », in P. BONTE \& M. IZARD et al., Dictionnaire de l'ethnologie et de l'anthropologie, Paris, P. U. F., 1991.

JARVIE, I. C., The Revolution in Anthropology, Chicago, Gateway edition, 1969.

JAUFFRET, L.-F., Les charmes de l'enfance et les plaisirs de I'amour maternel, Paris, Moutard, 1791.

JORION, P., «Aux origines de l'anthropologie française », L'Homme, $X X, 1980$, pp. 91-98.

KILANI, M., Introduction à I'anthropologie, Lausanne, Payot, 1992.

KILBORNE, B., « Le sauvage et I'homme », L'Ethnographie, fasc. $n^{\circ}$ 1, 1980, pp. 43-51.

KROEBER, T., Ishi. Testament du dernier Indien sauvage de l'Amérique du Nord, Paris, Plon, 1971.

LABILLARDIERE, J., Relation du voyage à la recherche de Lapérouse, Paris, 1796, 2 vol.

LABURTHE-TOLRA, PH. \& J.-P. WARNIER, Ethnologie, anthropologie, Paris, P. U. F., 1993.

[210]

LACÉPEDE, B., Cours de zoologie, discours d'ouverture, Paris, Plasson, an VIII [1799].

LANE, H., L'Enfant sauvage de l'Aveyron, Paris, Payot, 1979.

LAPLANTINE, F., Clefs pour I'anthropologie, Paris, Seghers, 1987.

LEBLOND, A.S., De l'instruction par les yeux, Paris, Imprimerie Sobry, 1799.

LECLERC, G., Anthropologie et colonialisme, Paris, Fayard, 1972.

LECLERC, G., L'Observation de I'homme, Paris, Le Seuil, 1979. 
LEIRIS, M., Journal 1922-1989, Paris, Gallimard, 1992, (édition de Jean Jamin).

LÉVI-STRAUSS, C., Les Structures élémentaires de la parenté, Paris, Mouton, 1967.

LOWIE, R. H., The History of Ethnological Theory, New York, Farrar and Rinehart, 1937, (traduction française: Paris, Payot, 1971).

MAGASIN ENCYCLOPÉDIQUE (dirigé par Millin), années 1799 à 1805.

MALSON, L., Les Enfants sauvages, mythe et réalité, suivi de Victor de I'Aveyron par J. Itard, Paris, U.G.E., 1964.

MANNONI, O., Clefs pour l'imaginaire ou l'Autre Scène, Paris, Seuil, 1969.

MARX, K., L'Idéologie allemande, Paris, Éditions sociales, 1968, (édition de Gilbert Badia). [Livre disponible dans Les Classiques des sciences sociales. JMT.]

MAUPERTUIS, P.-L., Lettre sur le progrès des sciences, Paris, 1752.

MAUSS, M., Manuel d'ethnographie, Paris, Payot, 1967. [Livre disponible dans Les Classiques des sciences sociales. JMT.]

MERCIER, P., Histoire de I'anthropologie, Paris, P.U.F., 1966.

METZGER, H., La Méthode philosophique en histoire des sciences. Textes 1914-1939, Paris, Fayard, 1987, (édition de Gad Freudenthal).

MÉTRAUX, A., «Les précurseurs de l'ethnologie en France du XVIe au XVIIIe siècle», Cahiers d'histoire mondiale, vol. VI, $\mathrm{n}^{\circ} 3$, 1963, pp. 721-738.

MINGUET, Ch., Alexandre de Humboldt, historien et géographe de l'Amérique espagnole (1799-1804), Paris, Maspéro, 1969.

MOORE, F.T.C., (éd.), The Observation of Savage People by J.-M. de Gérando, Berkeley, University of California Press, 1969. (Préface de E. E. Evans-Pritchard.) 
MORAVIA, S., Il Tramonto dell'illuminismo. Filosofia e politica nella societa francese (1770-1810), Bari, Laterza, 1968.

MORAVIA, S., La Scienza dell homo nel stettencento, Florence, La nuova Italia, 1970.

MORAVIA, S., Il pensiero degli ideologues, Florence, La nuova Italia, 1974.

MORGAN, L. H., Circular in Reference to the Degrees of Relationship among Différent Nations, Smithsonian Miscellaenous Collections, 2 (10), 1862.

MÜHLMANN, W., Geschischte der Anthropologie, Bonn, Universitats-Verlag, 1948.

[211]

NICOLET, C., L'Idée républicaine en France. Essai d'histoire critique, Paris, Gallimard, 1982.

NOTES AND QUERIES ON ANTHROPOLOGY, Londres Routledge \& Kegan Paul, 1951 (6e édition).

PALLAS, P.S., Voyages en différentes provinces de l'empire de Russie et dans l'Asie septentrionale, Paris, Lagrange, 1788-1793, 5 vol.

PELTO, P. J. et G. H., « Ethnography: the Fieldwork Entreprise», in J. Honigman (éd.), op. cit., 1973, pp. 241-288.

PENNIMAN, T. K., A Hundred Years of Anthropology, Londres, Duckworth, 1935.

PÉRON, F., Observations sur l'anthropologie.... Imprimerie de Stoupe, 1800.

PÉRON, F., Voyages de découvertes aux terres australes, Paris, Imprimerie impériale, 1807, T. I.

PÉRON, F., L. C. FREYCINET, Voyages de découvertes aux terres australes, Paris, Imprimerie royale, 1816, T. Il et Atlas historique. 
PICAVET, F., Les Idéologues. Essai sur /'histoire des idées et des théories scientifiques, philosophiques, religieuses, etc., en France depuis 1789, Paris, Alcan, 1891.

PINEL, PH., Traité médico-philosophique sur l'aliénation mentale ou la manie, Paris, Richard, Caillé et Rouvier, 1801, 318 p.

PINEL, PH., Nosographie philosophique, Paris, Brosson, 1813, 3 vol.

PLOMLEY, N.J.B., "The Baudin Expedition and the Tasmanian Aborigines in Tasmania », in Les Français et l'Australie, Paris, Université Paris X-Nanterre, 1989, pp. 133-140.

POIRIER, J., « Histoire de la pensée ethnologique », in J. Poirier, (éd.), Ethnologie générale, Paris, Gallimard, 1968, pp. 3-179.

POIRIER, J., Histoire de l'ethnologie, Paris, P.U.F., 1969.

POSTEL, J., Genèse de la psychiatrie. Les premiers écrits de Philippe Pinel, Paris, Le Sycomore, 1981.

QUATREFAGES, A., "Anthropologie», in Société de géographie. Instructions générales aux voyageurs, Paris, Delagrave 1875, pp. 244258.

QUATREFAGES, A., Hommes fossiles, hommes sauvages. Etudes d'anthropologie, Paris, Baillères, 1884 (réimpression Paris, JeanMichel Place, 1988, préface de Marika Moisseeff).

QUENTIN, P., Les Origines de l'idéologie, Paris, Economica, 1987.

REBOUL, R.M., Louis-Francois Jauffret, sa vie et ses ceuvres, Paris, Six, 1869.

REBOUL, R.M., Correspondance inédite de L-F. Jauffret, Draguignan, 1874.

RÉGALDO, M., Un milieu intellectuel: La Décade philosophique (1794-1807), Université de Lille, 1976.

[212] 
RÉGNIER, E., « Description et usage du dynamomètre », Journal de I'École polytechnique, 5e cahier, 1796, pp. 160-172.

RÉGNIER, E., «Mémoire sur le dynamomètre», Bulletin de la Société d'encouragement pour /'industrie nationale, $n^{\circ} 156,1817$, pp. 133-147.

ROGNON, F., Les Primitifs, nos contemporains, Paris, Hatier, 1988.

RUPP-EISENREICH, B., «The "Société des observateurs de I'homme" and German ethno-anthropology at the end of the 18th Century », History of Anthropology Newsletter, 10 (1), 1983, pp. 5-11.

RUPP-EISENREICH, B., «Cristoph Meiners et Joseph-Marie de Gérando: un chapitre du comparatisme anthropologique», in D. DROIXHE et P.-P. GOSSIAUX (éds), L'Homme des Lumières et la découverte de I'autre, Bruxelles, éditions de I'Université de Bruxelles, 1986, pp. 21-47.

SCHULTE-TENCKHOFF, I., La Vue portée au loin. Une histoire de la pensée anthropologique, Lausanne, Éditions d'En-bas, 1985.

SICARD, Abbé R., Théorie des signes.., Paris, Michaud, 1814, 2 vol.

SPENCER, H., An Autobiography, New York, Appleton, 1904. [Version française disponible dans Les Classiques des sciences sociales sous le titre: Autobiographie. Naissance de l'évolutionisme libéral. $\mathrm{J}^{\prime} M T$.]

STOCKING, G. W., «French Anthropology in 1800 », Isis, vol. LV, $2, n^{\circ} 180,1964$, pp. 134-150.

STOCKING, G. W., « Anthropology», in W. F. BYNUM, E. J. BROWNE \& Roy PORTER (eds), Dictionary of the History of Science, Londres, The Macmillan Press, 1983.

SWAIN, G., Le Sujet de la folie, Toulouse, Privat, 1977.

TODOROV, T., Nous et les autres. La Réflexion française sur la diversité humaine, Paris, Le Seuil, 1989. 
TOPINARD, P., «L'Ethnographie en 1800 », Revue d'anthropologie, $2 e$ série, $n^{\circ} 6,1883$, pp. 152-182. [Présentation et publication du mémoire de de Gérando: Considérations sur les diverses méthodes à suivre dans I'observation des peuples sauvages].

TYLOR, E. B., Anthropology, Londres, Ann Arbor Paperback 1960.

VAN GENNEP, A., Religion, moeurs et légendes. Essais d'ethnographie et de linguistique, Paris, Maisonneuve et Larose, 1914, 5 vol.

VERNES, J., Les Grands navigateurs du XVIIIe siècle, Paris, Ramsay, 1977 (édition originale : 1879).

VOGET, F. W., A History of Ethnology, Londres, Holt, Rinehart and Winston, 1975.

VOLNEY, C.-F., Tableau du climat et du sol des États-Unis, Paris Firmin Didot, 1846 (Ire édition : 1803).

Fin du texte 